Evaluating the Effects of the Physical Environment on Occupants using PostOccupancy Building Evaluation Data

\author{
by \\ Junmeng Liu \\ Honours BASc (Civil Engineering) \\ University of Waterloo 2017 \\ A thesis \\ presented to Ryerson University \\ in partial fulfillment of the \\ requirements for the degree of \\ Master of Applied Science \\ in the program of \\ Building Science
}

Toronto, Ontario, Canada, 2019

(C) Junmeng Liu, 2019 


\section{Author's Declaration for Electronic Submission of a Thesis}

I hereby declare that I am the sole author of this thesis. This is a true copy of the thesis, including any required final revisions, as accepted by my examiners.

I authorize Ryerson University to lend this thesis to other institutions or individuals for the purpose of scholarly research.

I further authorize Ryerson University to reproduce this thesis by photocopying or by other means, in total or in part, at the request of other institutions or individuals for the purpose of scholarly research.

I understand that my thesis may be made electronically available to the public. 


\title{
Evaluating the Effects of the Physical Environment on Occupants using Post- Occupancy Building Evaluation Data
}

Junmeng Liu

Master of Applied Science (2019)

Building Science Program - Ryerson University

\begin{abstract}
The physical built environment has long been an area of interest for researchers and can have large impacts on occupants in terms of their satisfaction and productivity. This research thesis utilizes post-occupancy data in occupied buildings to examine the effects of the physical workstation environment and the broader building amenities on occupants. Multi-variate regression models were used to analyze the data, taking into account demographic and workstation factors, the physical workstation environment, and satisfaction with amenity features. While the explained variance in the regression models were generally small, the results showed significant effects of various demographic factors, workstation characteristics, and physical workstation parameters on components of occupant satisfaction. Analyses of satisfaction with the broader building amenities further illustrated that elements outside of the immediate workstation can influence occupant satisfaction, well-being, and organizational productivity.
\end{abstract}




\section{Acknowledgements}

This thesis was an incredible journey for me, and I would like to express my sincerest appreciation to those who have supported and encouraged me along the way.

First, I would like to thank my thesis supervisor Dr. Mark Gorgolewski for his continued guidance and support throughout the thesis project. I am grateful for the independence I was given to pursue my interests, as well as the insight provided to me during this entire process.

Next, I would like to offer Dr. Guy Newsham and Dr. Jennifer Veitch my sincerest gratitude for their mentorship and advice throughout the course of this degree. This thesis would not have been possible without their feedback and patience in answering my many questions.

Finally, I would like to thank my family and friends for providing me with their encouragement throughout. 


\section{Table of Contents}

Author's Declaration for Electronic Submission of a Thesis................................................... ii

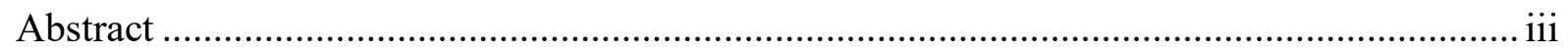

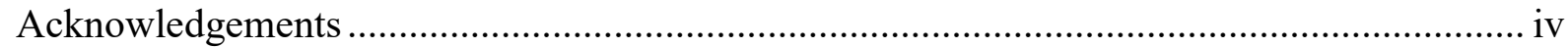

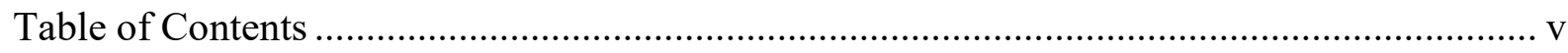

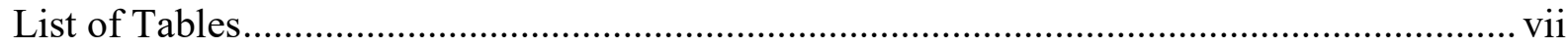

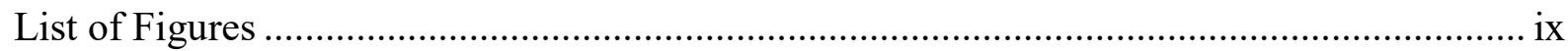

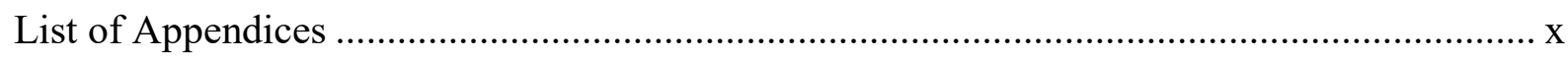

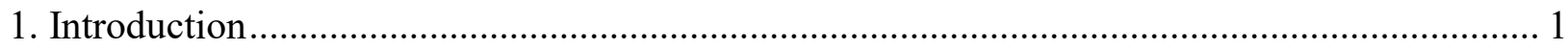

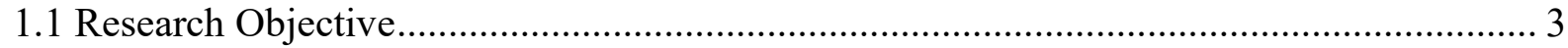

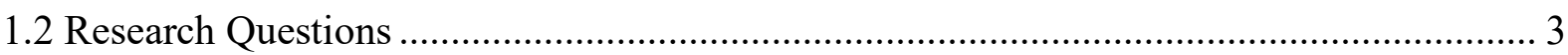

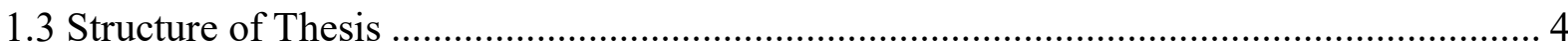

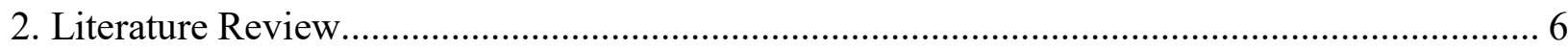

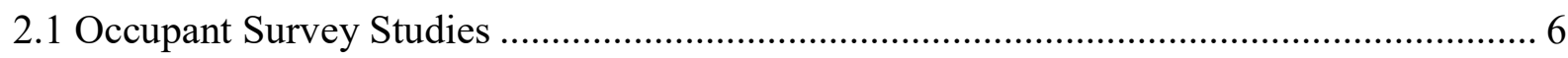

2.2 Studies Utilizing Occupant Surveys and General Physical Measurements ......................... 9

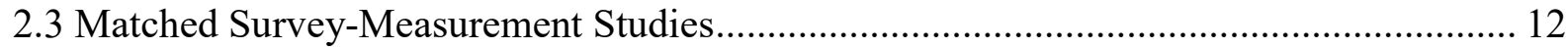

2.4 Studies of Functional Features and Building Amenities Outside of the Workstation ....... 14

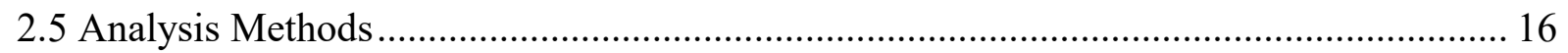

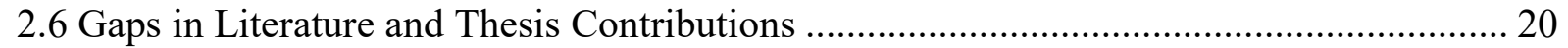

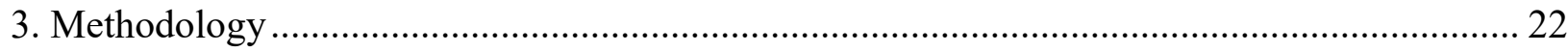

3.1 Analysis of the Physical Workstation Environment ........................................................ 23

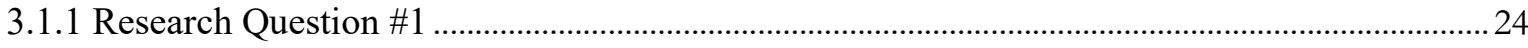

3.1.2 Research Question \#2 ...............................................................................................................27

3.2 Analysis of Amenities Outside of the Immediate Workstation (Research Question \#3)... 29

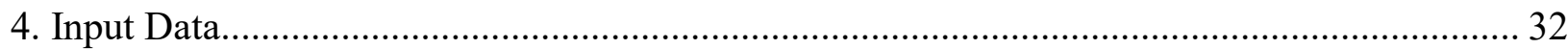

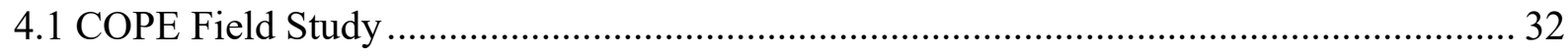

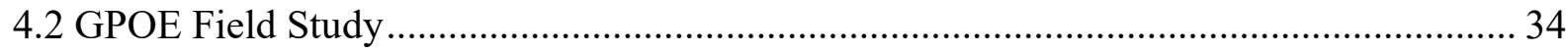

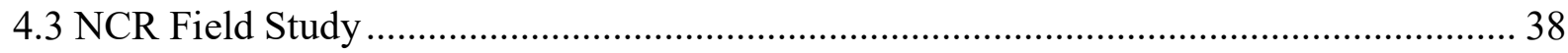

4.3.1 Setting ……………………………………………………………………………………..... 38

4.3.2 Occupant Survey and Physical Measurements ................................................................................. 39

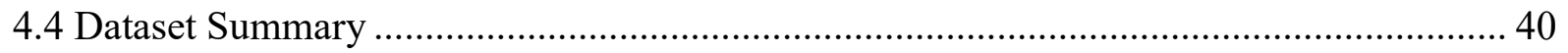




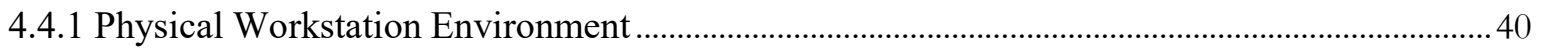

4.4.2 Broader Amenities Outside of the Immediate Workstation ............................................................. 44

5. Physical Workstation Conditions - Results .................................................................... 46

5.1 Descriptive Statistics and Variable Set-up ............................................................ 46

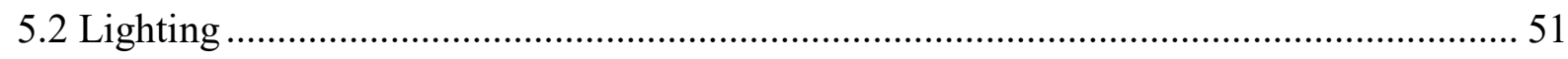

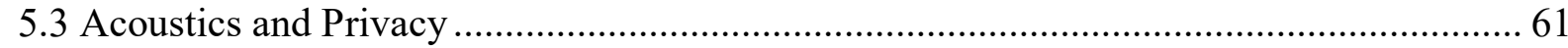

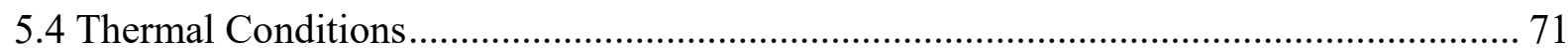

5.5 Discussion - Physical Workstation Conditions ....................................................... 81

6. Broader Amenities Outside the Workstation - Results .................................................... 88

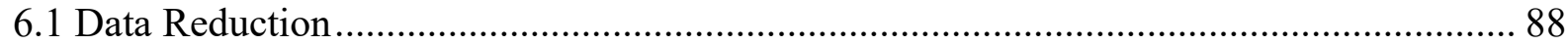

6.2 Descriptive Statistics and Variable Set-up ............................................................ 91

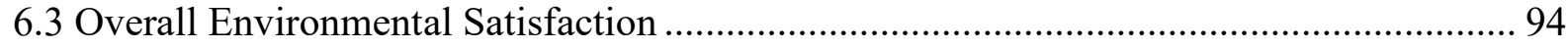

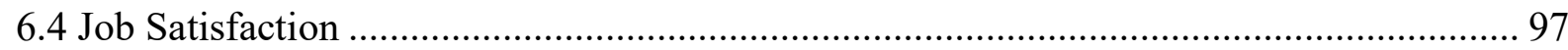

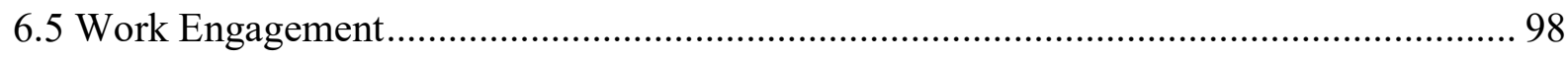

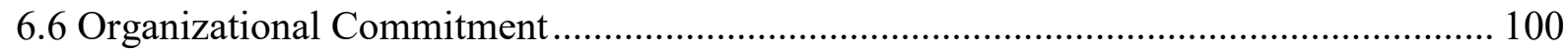

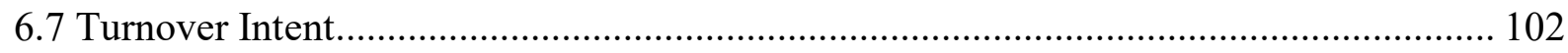

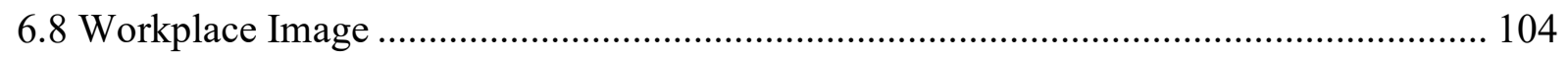

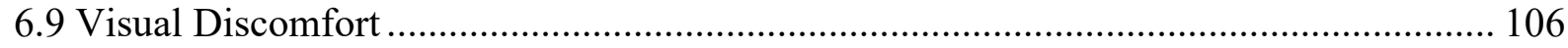

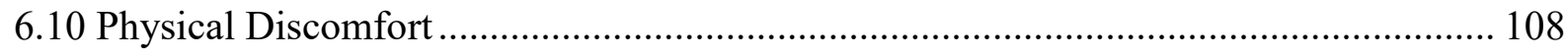

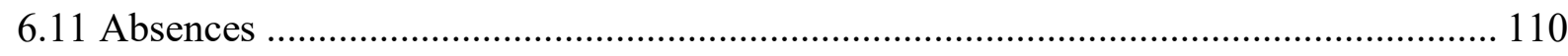

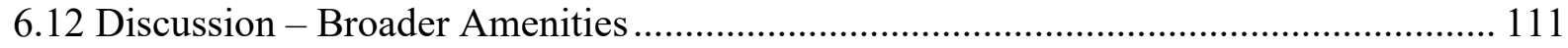

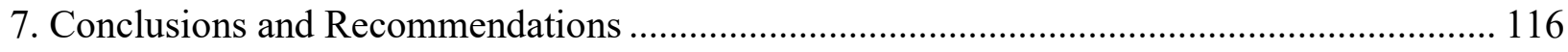

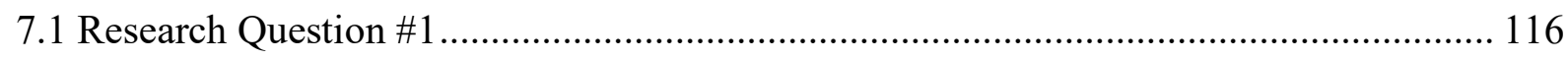

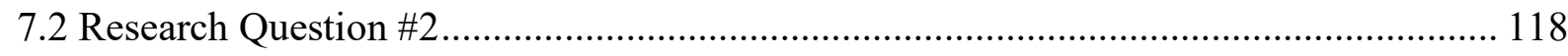

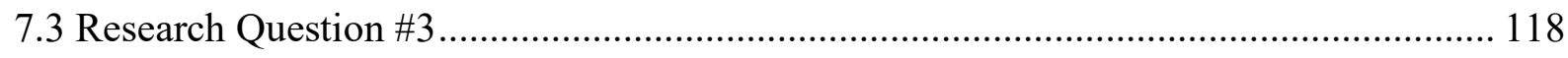

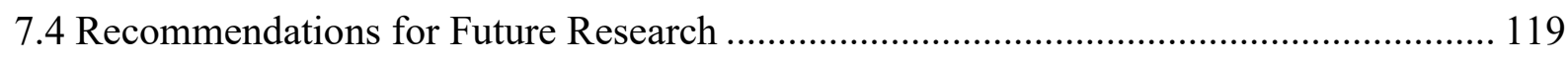

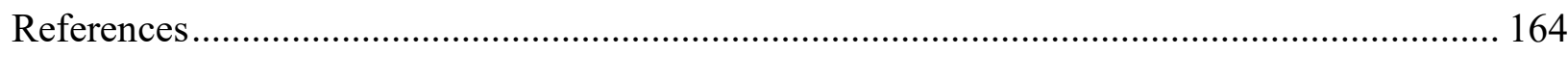

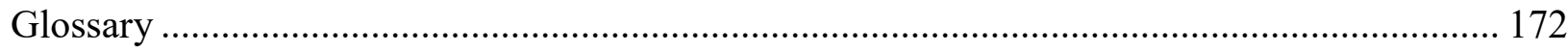




\section{List of Tables}

Table 1 - List of standards/guidelines and their recommended range........................................ 27

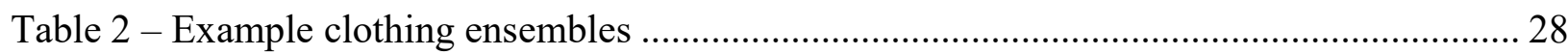

Table 3 - Outcome variables and their sources in literature....................................................... 30

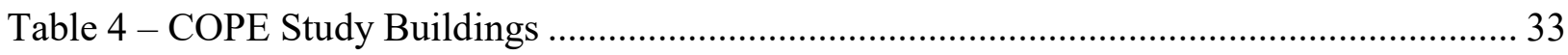

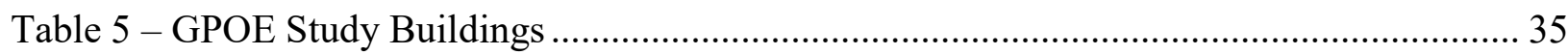

Table 6 - Summary of questionnaire modules in GPOE field study ………………………....... 38

Table 7- Summary of questionnaire modules in the NCR field study ......................................... 40

Table 8 - Instrumentation used for physical data capture ........................................................ 42

Table 9 - Demographics characteristics of the two data groups .................................................. 44

Table 10 - Demographics characteristics of the NCR respondents............................................. 45

Table 11 - Descriptive statistics of IEQ satisfaction measures (matched samples) …………...... 47

Table 12 - Descriptive statistics of physical measured parameters (matched samples)............... 48

Table 13 - Summary of the raw predictor variables (matched samples) ....................................... 49

Table 14 - Summary of sub-grouped satisfaction variables (matched samples).......................... 50

Table 15 - Categorization of IEQ reference variables (matched samples) ……............................. 51

Table 16 - Satisfaction with lighting regression results ............................................................. 53

Table 17 - Sub-grouped (asymmetric) lighting regression results ............................................. 56

Table 18 - Satisfaction with lighting regression results for IEQ guidelines ................................5 58

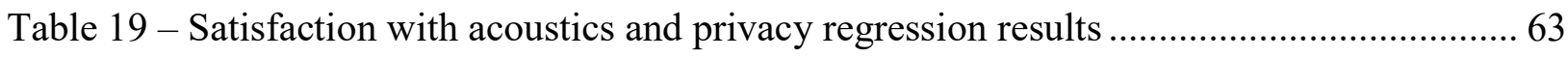

Table 20 - Sub-grouped (asymmetric) acoustics and privacy regression results ........................... 66

Table 21 - Satisfaction with acoustics and privacy regression results for IEQ guidelines .......... 69

Table 22 - Satisfaction with ventilation and temperature regression results................................ 74

Table 23 - Sub-grouped (asymmetric) ventilation and temperature regression results................. 77

Table 24 - Satisfaction with ventilation and temperature regression results for IEQ guidelines. 79

Table 25 - Composite amenities satisfaction scales ............................................................. 90

Table 26 - Descriptive statistics for environmental and amenities satisfaction predictor variables

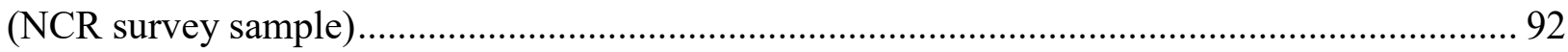

Table 27 - Descriptive statistics for outcome variables (NCR survey sample) …………............ 93

Table 28 - Description of the predictor variables (NCR survey sample)...................................... 94

Table 29 - Overall environmental satisfaction regression........................................................ 95 


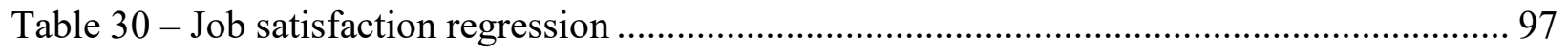

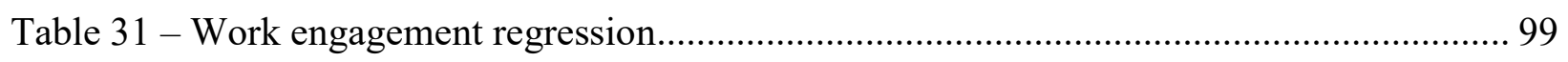

Table 32 - Organizational commitment regression ............................................................... 101

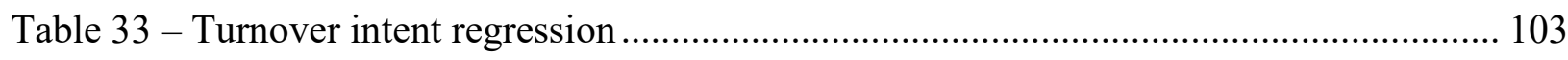

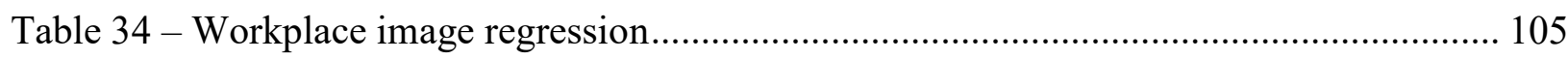

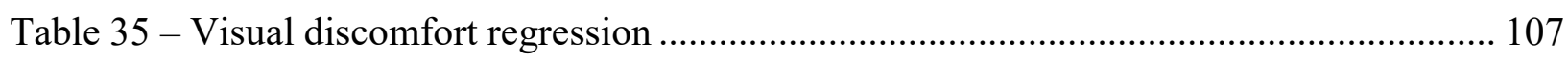

Table 36 - Physical discomfort regression ........................................................................... 109

Table 37 - Absences due to illness regression.......................................................................... 111 


\section{List of Figures}

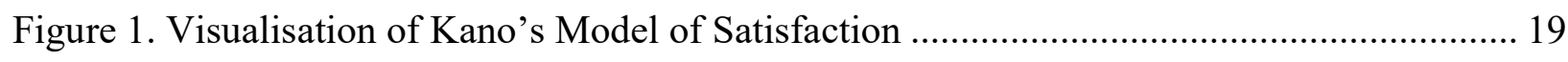

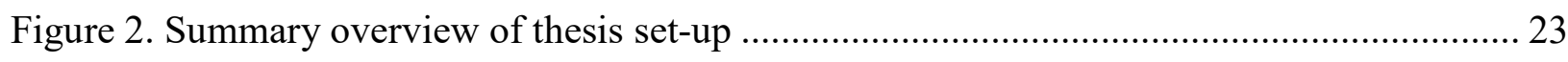

Figure 3. Visualisation of a basic factor using the three-line regression model set-up ................ 26

Figure 4. Visualisation of a bonus factor using the three-line regression model set-up ............... 26

Figure 5. Cart and chair used for physical IEQ measurements in the COPE study.......................34

Figure 6. NICE Cart used for physical IEQ measurements in the GPOE and NCR studies ........ 36

Figure 7. In-situ schematic diagram of NICE Cart .............................................................. 37

Figure 8. Satisfaction with lighting vs. desktop illuminance..................................................... 52

Figure 9. Satisfaction with acoustics and privacy vs. background sound level............................. 62

Figure 10. Satisfaction with acoustics and privacy vs. speech intelligibility ................................ 62

Figure 11. Satisfaction with ventilation and temperature vs. air temperature .............................. 72

Figure 12. Satisfaction with ventilation and temperature vs. relative humidity ........................... 72

Figure 13. Satisfaction with ventilation and temperature vs. radiant temperature ……................ 72

Figure 14. Satisfaction with ventilation and temperature vs. air velocity .................................... 73 


\section{List of Appendices}

APPENDIX A - Church Street Development Project Report...............................122

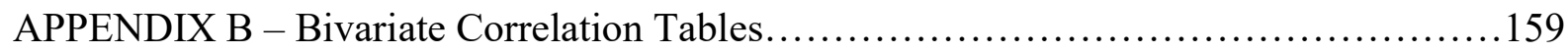

APPENDIX C - Principal Components Analysis Tables..................................162 


\section{Introduction}

In developed economies, people spend approximately $90 \%$ of their lives indoors (Klepeis et al., 2001; Leech, Nelson, Burnett, \& Raizenne, 2002). The on-going shift in recent years where the global workforce is moving to more service and knowledge-based sectors has only exacerbated this effect (World Green Building Council, 2014). These sectors largely operate in indoor office environments, resulting in greater emphasis on indoor environment quality (IEQ) and the subsequent comfort and well-being of occupants. Consequently, these areas are receiving increasing attention in both research and industry.

It has been well established in literature that aspects of the physical workspace have an effect on job satisfaction, stress levels, and health (Klitzman \& Stellman, 1989; Pejtersen, Allermann, Kristensen, \& Poulsen, 2006). A well-conditioned indoor environment plays a crucial role in achieving higher levels of organizational productivity and well-being (World Green Building Council 2014). In terms of economic importance, existing studies have demonstrated the significant financial benefits of improved IEQ (Fisk, Black, \& Brunner, 2011). The economic significance of occupants is further emphasized by Brill, Weidemann, and BOSTI (2001), who estimate that over a ten year period, people (i.e. occupants) make up roughly $82 \%$ of an organization's costs. Given the demonstrated financial importance of the occupants, it is of interest to building owners and organizations to better understand the links between the physical environment and occupant satisfaction.

Traditionally, field studies involving in-situ building performance and occupant feedback have been achieved through a process called post occupancy evaluations (POE). Fundamentally, POEs are a process that involves the evaluation of buildings in a systematic manner after they 
have been built and occupied for some time (Preiser \& Vischer, 2005). This involves assessing how the building performs after being occupied and how satisfied the users are with the environment that has been created. However, building POEs are still an emerging field that often goes unfunded. The cause of this has been attributed to the fact that these studies are perceived as 'more of the same' (Leaman, Stevenson, \& Bordass, 2010). A significant portion of available literature in the domain of POEs rely solely on occupant survey data and do not incorporate quantitative physical measurements of the indoor environment (e.g. temperature, relative humidity). Studies that do report physical measures of the indoor environment very often do so in a general sense, reporting means and standard deviations and comparing them against published industry standards for compliance. By not having matched comparisons between an occupants' response and the physical measured conditions of their workspace, results from these investigations are often qualitative and specific to the research building(s). This places a severe restriction on the ability to extract useful lessons-learned. Results such as 'occupants were generally unsatisfied with lighting conditions' based on occupant questionnaire responses are not particularly insightful for buildings other than those directly involved in the study.

Given the demonstrated significance of the physical environment in terms of both economic benefits and occupant well-being, further research into the area is worthwhile. The aim of this thesis is to better understand the links between the various aspects of the physical built environment on occupant satisfaction and productivity. This thesis seeks to contribute to the POE body of knowledge by utilizing the large quantity of cross-sectional field data gathered by the National Research Council Canada (NRC) across three large scale building studies. 


\subsection{Research Objective}

The overall research objective of this thesis is to examine how the various aspects of the physical built environment affects occupant satisfaction and productivity in an office setting. The study seeks to contribute to the field by addressing some of the gaps in the literature, and to further verify and compare against the results of other researchers using different analysis protocols. This study takes advantage of new, recently collected data from a large building evaluation field study in combination with the large quantity of existing data gathered in the last two decades by the NRC. Matched physical and occupant survey datasets of this size are rare, particularly when many facets of the indoor environment are considered simultaneously. The most recent building evaluation study also extends the scope of the survey, providing information on the broader building amenities.

The aim is to contribute to the current body of knowledge through a unique analysis of the new and existing data, and to expand upon the scope of existing POE studies by incorporating the effects of elements outside of the immediate workstation. The results of this thesis will help industry practitioners and researchers to better understand the links between the physical environment and satisfaction, leading to higher performing and well functioning spaces and organizations.

\subsection{Research Questions}

The specific research questions of this thesis are as follows:

- How do aspects of the physical workstation conditions and indoor environment affect occupant satisfaction? Both the proportional (linear) effects, as well as the asymmetric (non-linear) effects of these factors on satisfaction will be explored. 
- To what extent does compliance with published IEQ standards and reference criteria improve occupant satisfaction? Within this research question, North American standards and guidelines, to which these buildings would be expected to conform, will be examined to evaluate their impact on occupant environmental satisfaction.

- What are the effects of the broader building amenities outside of the immediate workstation on satisfaction and organizational productivity metrics, and how do they compare to the influence of the workstation environment? This research question is answered utilizing the newest building evaluation study, which employs more detailed survey items relating to amenities and site facilities.

\subsection{Structure of Thesis}

The research thesis will be structured into two distinct, but complementary sections: evaluation of (1) the physical workstation conditions, and (2) the broader building amenities outside of the immediate workstation. Within the analysis of the physical workstation conditions, the first two research questions will be explored. Each component of the indoor environment (e.g. lighting, acoustics) will be analyzed separately, first for the effects of various measured IEQ parameters, and then for their conformance with relevant standards and reference values. The third research question concerning amenities will be explored in its own section of the thesis.

While this thesis utilizes existing data collected by others (i.e. NRC), similar research projects requiring field data collection and interpretation have been performed for the Church Street Development Project at Ryerson University. The data collected for this project did not contribute directly to answering the thesis' research questions, however it did provide added insight and understanding of post-occupancy evaluation procedures. The project provided valuable knowledge of both the physical and survey data collection processes, as well as the data 
extraction and reporting procedures. An example of a lesson learned from this process is the importance of proper documentation and manual recording of workstation features not captured by the sensors. Features like task lighting, shading devices, and personal heaters, can all play a central role in an occupant's use of space and affect their subsequent satisfaction. A copy of this project report can be found in Appendix A. 


\section{Literature Review}

A literature review of existing building studies and POEs is conducted to compile the current evidence on how the physical environment affects occupant satisfaction. The review is largely grouped by the source of data: studies using only occupant surveys, studies using occupant surveys and general physical measurements, and studies using matched occupant survey and physical measurements. A separate section is included to address studies of building features and amenities outside of the immediate workstation. A final section summarizing some analysis procedures that have been used by others, as well as outlining some gaps in the literature is included at the end.

\subsection{Occupant Survey Studies}

Studies utilizing occupant survey responses as the primary source of data are the most prevalent way to perform a POE. These studies typically require less resources, and often have large sample sizes as survey responses can be easily stored and shared in databases amongst researchers. This facilitates sharing of data and allows researchers to pick and choose certain study populations (with large sample sizes) based on desired building/occupant attributes and research objectives. An example of such a database is the Centre for the Building Environment (CBE) survey database. Occupant surveys are an established POE method used to examine the influence of different building and workstation characteristics on occupant satisfaction.

To date, a number of available literature evaluate the effects of open workspaces on occupants. In a study of 22 office buildings, Pejtersen et al. (2006) found that occupants in openplan offices are more likely to report issues with health and poor air quality and experience thermal and acoustic discomfort. Similar findings were reported by Kim and de Dear (2013) using the CBE survey database, where enclosed private offices performed higher than open-plan 
workstations in terms of acoustics and privacy. They also argued that the benefits of 'ease of interaction' in open-plan work environments were smaller than the drawbacks of the increased noise level and decreased privacy. Other literature, (e.g. Otterbring et al. 2018; Frontczak et al. 2012) generally support the notion that more open workstations result in lower job satisfaction and environmental comfort. Still, some studies have found conflicting evidence. In a study of 15 LEED-certified buildings, Lee (2010) found that people in cubicles with high partitions were significantly less satisfied with noise level and privacy. The same study also found that participants in workstations with high partition walls had lower job performance than both enclosed private offices and open plan workstations without partitions. Further, they found that open workstations with no partitions reported significantly higher acoustic quality than workstations with partitions. Brown et al. (2010) also found higher environmental satisfaction in open plan offices in a pre-and-post relocation study, though these observed effects are likely the result of moving from a "conventional" building to a "green" building with other positive features.

POE studies utilizing occupant surveys have also investigated other aspects of workplace perceptions like access to natural light. Yildirim, Akalin-Baskaya, and Celebi (2007) found window access improved employee satisfaction, compensating for the negative aspects of openplan workstations. A number of existing studies support this result, and consistently report the positive effects of window proximity and daylighting on satisfaction and comfort (Aries, Veitch, \& Newsham, 2010; Elzeyadi, 2011; Frontczak et al., 2012).

Demographic differences have also been shown to play a role in terms of occupant satisfaction and comfort. With regards to gender differences, studies have generally shown women to experience higher levels of dissatisfaction with their workstation environment than 
their male counterparts (Bluyssen, Aries, \& van Dommelen, 2011; Karjalainen, 2007; Kim, de Dear, Cândido, Zhang, \& Arens, 2013; Yildirim et al., 2007). Researchers have also shown that younger occupants tend to be more dissatisfied with their work conditions than older occupants (Bluyssen et al., 2011; Klitzman \& Stellman, 1989).

Using occupant questionnaire data, researchers have also tried to link elements of IEQ to satisfaction and performance. Altomonte et al. (2017), using survey data from 93 buildings in the CBE database, found that achieving LEED IEQ credits (EQc credits, LEED v3 or prior) relating to aspects of the indoor environment did not lead to more satisfied occupants. While this study questions the effectiveness of prescribed LEED IEQ credits, the importance of IEQ is welldocumented in literature. Klitzman and Stellman (1989) found that physical work conditions have substantial effects on the psychological well-being of occupants, more than demographics or occupational factors. The same paper also suggests that air quality and noise were the most important aspects of the environmental satisfaction. This result is broadly supported by ZalejskaJonsson and Wilhelmsson (2013), who found air quality to be the most impactful aspect of IEQ satisfaction, followed by thermal comfort, then sound quality last. Other researchers have also found, to varying degrees, the influence of individual components of IEQ satisfaction on overall occupant satisfaction (Kim \& de Dear, 2012; Martellotta, Simone, Della Crociata, \& D’Alba, 2016). Studies have further reported environmental satisfaction to have a significant effect on workplace productivity (Leaman, 1995).

Yet, Huizenga et al. (2006) reported that buildings generally do not meet the IEQ performance goals set out by industry standards in terms of thermal comfort and air quality satisfaction. This result outlines the performance issues and need for improvement in current buildings. Furthermore, while the importance of IEQ are well-established, studies suggest that 
overall comfort is much more than the average of perceived indoor air quality, noise, lighting and thermal comfort responses (Bluyssen et al., 2011). These results highlight the complexity of occupant comfort and IEQ, and that more research in the field is necessary.

\subsection{Studies Utilizing Occupant Surveys and General Physical Measurements}

A significant portion of existing POE studies also supplement occupant surveys with the use of physical measured IEQ data. In these research studies, physical measurements are typically taken at representative locations across the sample buildings. However, potentially due to anonymity or other issues, the measurements are not traced back to the questionnaire respondent at the measurement locations. Many of these studies report similar conclusions to those utilizing only survey data, with the physical measurements providing additional context.

In a post-occupancy study of three Malaysian buildings, Ali, Chua, and Lim (2015) found aspects of physical environmental satisfaction, specifically lighting and room temperature, to be significantly correlated to physical and visual discomfort and health-related issues. This paper's use of physical IEQ measurements was limited to reports of the mean as well as compliance with local prevailing standards for lighting and temperature. There are other studies which have found higher temperatures to be associated with warmer perceptions, but these perceptions did not result in decreased satisfaction (Lakeridou, Ucci, Marmot, \& Ridley, 2012). Pollutant concentrations have also been shown to have a significant influence on air quality satisfaction, with lower measured $\mathrm{CO}_{2}$ concentrations reporting higher satisfaction (Wang et al., 2015). The same study also reports thermal environment and air quality to be the most important aspects of IEQ, in agreement with other POE studies (e.g. Zalejska-Jonsson and Wilhelmsson 2013;

Klitzman and Stellman 1989). 
In terms of lighting, Hwang and Jeong (2011) found improvements to occupant health and productivity from daylighting, though sun-shading devices were recommended to reduce glare annoyance. Again, illuminance levels and uniformity were compared to recommended values stated in relevant standards and guidelines.

Some researchers have also performed longitudinal studies using physical measurements and occupant survey responses. In a study utilizing measurements from stationary sensors across several days, air temperature was found to be significantly correlated with self-estimated performance (Nematchoua, Ricciardi, Orosa, Asadi, \& Choudhary, 2018). The same study reported that temperatures above 28 degrees Celsius drastically reduced occupant performance, and that optimal performance occurred when the thermal sensation vote was neutral.

A number of existing literature also focussed on investigating the performance differences between green and conventional buildings in terms of satisfaction and IEQ. There is a general consensus among researchers that occupants are more satisfied in green buildings than conventional. However, claims of improved physical measured indoor environment quality in green buildings are less supported in literature. Pei et al. (2015) found that although occupants in green buildings were more satisfied, IEQ measurements like illuminance, temperature, and $\mathrm{CO}_{2}$ concentrations did not differ significantly compared to conventional buildings. The same study also compared IEQ measurements for compliance with local building standards to evaluate as an indication of acceptable performance. These results are largely in agreement with the findings of Liang et al. (2014). MacNaughton et al. (2017) found that occupants have better cognitive function and lower sick building syndrome (SBS) symptoms in green buildings than similar high performing conventional buildings, though they only partially attributed this to improved IEQ. Varied results were also reported by Lee et al. (2019). The study found lower temperature and 
relative humidity fluctuations, as well as lower concentrations of particulates (PM2.5) in green buildings, but no notable differences in other aspects of IEQ such as lighting or carbon monoxide. Conversely, Tham, Wargocki, and Tan (2015) found that green buildings had more brightly lit environments, and were less leaky than conventional buildings. While the study found that occupants were more satisfied in green buildings, there were no benefits observed benefits in terms of sick building syndrome (SBS) symptoms and sick leave. There are many possible reasons for why occupants are more satisfied in green buildings, even when IEQ measurements are similar. Some researchers like Holmgren, Kabanshi, \& Sörqvist (2017) have suggested that psychological associations with the green certification plays a key role. When occupants understand that they are situated in a green building, their perceptions are improved and they are more forgiving of indoor environment conditions.

In post-occupancy building research without direct, matched survey-measurement data, often only broad analyses of means and standard deviations of the physical measured IEQ factors are reported. For instance, Silva et al. (2017) performed a POE study where IEQ measurements were taken and measured against industry standards. However, the extrapolation and usefulness of the results beyond its application to the two study buildings is very limited, highlighting the limitations of studies of this type. These evaluations often compare the physical IEQ measurements against relevant standards as an indication of acceptable performance, but perform no further validation of their effects on occupant satisfaction (e.g. Liang et al. 2014; Pei et al. 2015; Kwon, Chun, and Kwak 2011). While these published standards and their recommended IEQ thresholds are based on consensus, derived from studies of human responses to physical conditions, they were primarily conducted in controlled laboratory conditions. Furthermore, each study generally focused only on a single aspect of the indoor environment. Many of the reviewed 
POE studies in this section also do not consider various confounding factors such as workstation characteristics that may affect the perception of raw physical IEQ measurements. For instance, comparing lighting/illuminance measurements to reference values without consideration for window proximity can be inappropriate, and studies have shown that natural lighting and artificial lighting are not perceived the same way by occupants (Galasiu \& Veitch, 2006). As such, additional research into the effectiveness of some of these published physical IEQ guidelines for their in-situ effectiveness in achieving occupant satisfaction is necessary.

\subsection{Matched Survey-Measurement Studies}

A much smaller subset of the available POE literature utilizes matched cross-sectional data of occupant responses and physical IEQ measurements. These types of studies, although more restricting and time-consuming in the collection of data, can provide much more insight into the relationships between the physical environment and perceptions of the occupant.

Several earlier research studies done by NRC were able to obtain significant results by matching occupant responses with measured data. In a study of open plan workstations, Charles et al. (2006) found that occupants were less satisfied with increasing workstation temperatures and air speeds. The same study also showed that higher carbon dioxide concentrations had negative effects on satisfaction. Veitch et al. (2005) found that lighting uniformity influences lighting satisfaction, with more uniformly lit spaces perceived more favourably. However, the actual measure of desktop illuminance, a commonly reference value in both design and practice, was not considered in the study. In a study using the top and bottom $20 \%$ of respondents of the original data sample, Newsham, Veitch, and Charles (2008) found that temperatures higher than the average measured indoor temperature, higher $\mathrm{CO}_{2}$ concentrations, desktop lighting outside of 300-500 lux, and high monitor glare (CRT) all negatively impacted aspects of environmental 
satisfaction. However, office spaces nowadays very rarely employ the use of CRT screens, and the results concerning screen glare are less relevant in today's workplaces. Also, while it can be argued that using the top and bottom $20 \%$ of respondents evaluates the most extreme effects in the data, willingly eliminating $60 \%$ of the sample size reduces the strength of the analyses and is not common practice. In a more recent study of two separate datasets, Leder et al. (2016) found associations between lighting satisfaction and CRT monitor reflections, lighting uniformity, and illuminances below 300 lux. The study also found that green buildings had significantly higher satisfaction in multiple aspects of environmental satisfaction given similar measured physical environmental conditions. However, beyond lighting, the study found few significant relationships between aspects of the measured workstation environment and occupant satisfaction. Furthermore, while two datasets were analyzed independently, the predictor variables used in the study were not consistent between the two datasets. There is very little justification of the reasoning for this in the paper and does not lead to consistent interpretation of the result; it is also possible that variables that best fit the regression results in each dataset were chosen.

Researchers at Carnegie Mellon University have also taken advantage of matched survey responses and physical measurements. Choi, Loftness, and Aziz (2012) found that workstations with higher measured desktop illuminance resulted in higher satisfaction with lighting on the desktop, but lower satisfaction with reflected light/glare. However, the analyses do not account for confounding factors such as window proximity in the lighting analysis. Window offices are expected to have higher lighting levels, and the benefits in satisfaction could be a result of window proximity, which has been shown to increase satisfaction, as opposed to actual lighting levels. The study also reported no differences in measured temperatures or satisfaction amongst 
the different genders and age groups, although females were less satisfied in the summer. The differences in thermal satisfaction between the genders, and the lack of differences between age groups are supported by other similar studies (Choi, Aziz, \& Loftness, 2010).

Other researchers such as Tanabe, Haneda, and Nishihara (2015) chose to limit the scope of their work to only the thermal environment. Although the relationships between air temperature and performance were found to be inconclusive in this study, there was a strong positive relationship found between thermal satisfaction and higher performance. This again illustrates the complexities of research in this field, as performance was correlated to perceived thermal satisfaction as opposed to actual measured temperatures.

\subsection{Studies of Functional Features and Building Amenities Outside of the}

\section{Workstation}

Studies in the realm of building science and POEs rarely incorporate the effects of amenities and factors beyond the occupants' immediate workstations in their analysis. However, researchers in the domains of behavioural science, environmental psychology, real estate, and architectural design have more routinely investigated the links between the broader building amenities and organizational performance. When investigated independently, the benefits of elements of amenity features are well-established.

In a study of facilities maintenance in Cameroon, Foju (1993) demonstrated that occupant perceptions of well-maintained services (e.g. restrooms, elevators) and office equipment had beneficial effects on environmental satisfaction and comfort. Vischer (2008) echoes this result, and proposed in a conceptual model of worker productivity that organizational effectiveness is influenced by building amenities such as fast elevators, convenient bathrooms, adequate parking, and attractive eating areas. This is supported by Lee and Kim (2014), who used confirmatory 
analysis and structural equation modelling to demonstrate that perceptions of public space cleanliness and ease of navigation both significantly affected user satisfaction and comfort.

Studies have further shown that not all amenities are worth the investment, and the presence of collaboration spaces (e.g. maker spaces and work cafes) and quiet places to perform focused work had the greatest value to occupants (Gensler, 2019). The same study also reported that amenities with a non-work focus such as break rooms or lounges had the smallest performance gains. Agha-Hossein et al. (2013) showed that satisfaction with interior use of space (e.g. meeting spaces, areas for socializing, and navigational signage) predicted self-reported productivity and well-being. They also found no relationship between indoor facilities (e.g. kitchen facilities and cycling facilities such as showers) and the outcome measures. A larger percentage of floor space dedicated to shared services/amenities and shorter distances from workstations to meeting rooms can also improve perceived collaboration in occupants (Hua, Loftness, Heerwagen, \& Powell, 2011).

There are also a number of existing literature that focus on investigation the workplace aesthetic choices contributing to a pleasant work environment. For instance, several studies have demonstrated the positive effects of biophilia and natural elements in the workplace on organizational productivity (Bergs, 2002; Gray \& Birrel1, 2014; MacKerron \& Mourato, 2013) and decrease absenteeism (Elzeyadi, 2011). Researchers have also found other on-site and proximate life convenience amenity features to contribute to occupant satisfaction in a professional setting. Barton (1992) found that on-site child care services improved employee retention and satisfaction, while Shephard (1992) reported similar positive effects of on-site fitness facilities. Data from several studies also suggest the importance of transportation facilities, where elements such as the presence of reserved parking (Getka, 2012) and perceptions 
of ease of access to public transit stations (Ettema et al., 2011) positively influenced occupant satisfaction.

\subsection{Analysis Methods}

Within the reviewed literature, several common methods of analyzing POE data were identified. This includes simple reporting of descriptive statistics (means, standard deviations) and $\mathrm{X}$ vs. Y graphs, to more complex statistical procedures like analysis of variance (ANOVA) and various multi-variate regression techniques. Many of the data analysis procedures used in the reviewed literature such as ANOVA do not consider the influence of confounding factors in the interpretation of the results. For instance, window proximity can reasonably be expected to influence the raw measured illuminance values. When studies report the benefits of higher illuminance without consideration for windows, the observed effects could be due to the unaccounted factor of windows and natural lighting as opposed to the increased illuminance. The same line of reasoning applies for other parameters like more enclosed workstations and acoustics, as well as gender differences in thermal satisfaction. As such, this section in the review will focus more on the multi-variate techniques that have been utilized in prior studies, which consider multiple variables within the same analysis.

Using matched survey-measurement data, researchers at NRC have used various multiple regression techniques to analyze the effects of the workspace on occupants. Using binary logistic regression (BLR), Newsham, Veitch, and Charles (2008) have explored the risk factors for occupant dissatisfaction. This type of analysis has the benefit of not requiring data to be normally distributed, or that data for all combinations of variables be present in all samples. The results are reported in terms of odds ratios, i.e., the chance of something being in one category versus another. For instance, a sample result from this study is that people with windows were about 
twice as likely to be in the very dissatisfied group compared to those who were distant from a window. There are benefits to this procedure in that the results can help define clear criteria through associations of various predictor variable categories. However, there are also some potential drawbacks. The main issue is that the dependent variable in BLR, typically the satisfaction measure in the context of POEs, needs to be categorical (e.g. dissatisfied versus satisfied). As such, there are reductions to the resolution of the satisfaction measures and can lead to skewing of the results. On a 7-point Likert type satisfaction scale $(1=$ very unsatisfied, 4 $=$ neutral, $7=$ very satisfied), a satisfaction response of 4.9 is not so different from a rating of 5.1, yet they can be in two distinct categories in BLR. Furthermore, the variance explained in BLR models is represented by pseudo $\mathrm{R}^{2}$, as opposed to the traditional percentage of variance explained in linear regression models. This inhibits the ability to compare with other studies utilizing linear regression models, which is a more prevalent technique used in POE studies.

Multiple linear regression has been used consistently across various studies utilizing matched survey-measurement data (Charles et al., 2006; Leder et al., 2016; Veitch et al., 2005). Specifically, hierarchical multiple regression was used to allow the researchers to control the input of variables in a fixed order on theoretical grounds. In general, these studies have input demographic factors as the first block in the regression model, followed by workstation characteristics, and finally the IEQ parameters. Linear regressions require normality in the data. The normality criteria used were skewness values between +3 and -3 and kurtosis values between +8 and -8 , to maintain consistency with previously published studies (Kline, 1997). Excessively intercorrelated variables are also generally avoided in the statistical models (Hair, Black, Bajin, \& Anderson, 2014). As the name suggests, these regression models only examine the linear relationships between the various predictor variables and the outcome satisfaction 
measures, which is a limitation in the procedure. However, there are also many benefits to this analysis method. For instance, the resolution of the satisfaction variable is not reduced by the need to categorize them (as in BLR). Multiple linear regressions also offer a straightforward relationship between the predictor and the outcome that is easily interpretable. Furthermore, the effect sizes of the models $\left(\mathrm{R}^{2}\right)$ can be generally be compared across multiple studies, and it is not subject to different calculation methods like pseudo $\mathrm{R}^{2}$.

Some studies utilizing only occupant surveys have also used multiple regression to investigate the nonlinear effects based on Kano's Model of Satisfaction (Kano, Seraku, Takahashi, \& Tsuji, 1984). This model was first applied to POE building research by Kim and de Dear (2012) in an analysis of occupant survey data. The premise behind Kano's model is that there are three types of influencing factors: basic factors, proportional factors, and bonus factors. A basic factor is one that is 'must-be' and are factors that, if missing, have a stronger effect on dissatisfaction. Basic factors can be thought of as minimum requirements, and their effects are only felt if deficient or lacking in some way. These factors do not necessarily enhance satisfaction but can cause dissatisfaction when not fulfilled. Proportional factors are 'linear' factors that contribute to both satisfaction and dissatisfaction. When these factors are performing well occupants are satisfied; when these factors perform poorly occupants are dissatisfied. Bonus factors are also referred to as 'attractive' factors and are factors that have a strong effect on an occupant's satisfaction. Lack of these factors do not necessarily result in dissatisfaction. A visual representation of this is shown below in Figure 1. The figure illustrates that in the case of basic factors, underperformance has a larger impact on the negative end of the satisfaction scale (i.e. dissatisfaction) than high performance does on the positive end. The reverse is true for bonus factors, where the effect of these factors is much greater in satisfied occupants. 


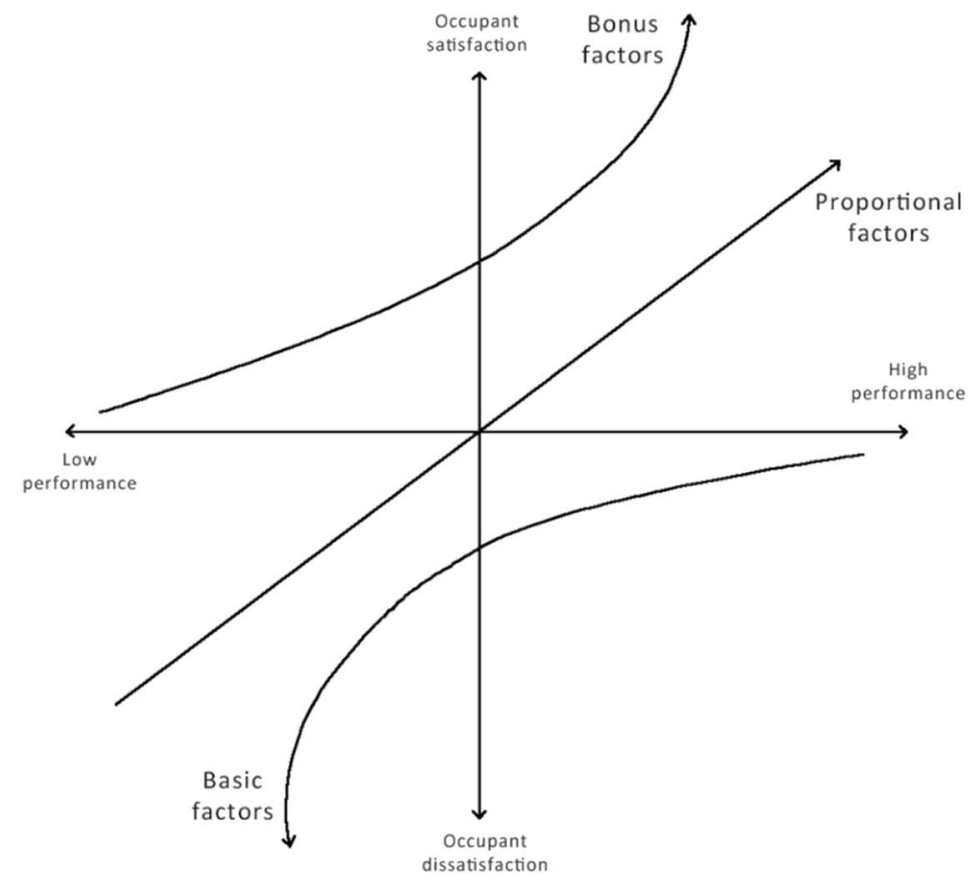

Figure 1. Visualisation of Kano's Model of Satisfaction (taken from Kim and de Dear, 2012)

The outcome variable in these previous POE research applications of Kano's model (e.g. Kim and de Dear 2012; Martellotta et al. 2016) using multiple regression was overall satisfaction. The predictor variables were the various individual aspects of environmental satisfaction, such as satisfaction with thermal comfort, lighting etc. The predictor variables were recoded into dummy variables based on whether the occupant response was in the dissatisfied range, neutral range, or the satisfied range. Using lighting as an example, a new 'lighting dissatisfied' variable was created, which had a dummy value of ' 0 ' if the respondent was not dissatisfied, and a value of ' 1 ' if the respondent was dissatisfied. Similarly, variables for 'lighting neutral' and 'lighting satisfied' variables were created. These dummy variables were then used as inputs into the multiple regression model to predict overall satisfaction. The rationale is that if the 'dissatisfied' dummy variable was a significant predictor but the 'satisfied' variable was not, that variable behaved as a basic factor. The reverse was used to identify bonus 
factors; if the 'satisfied' variable was a significant predictor but the 'dissatisfied' variable was not, then the variable behaved as a bonus factor.

Multiple regressions are a commonly chosen analysis procedure in the reviewed literature, with various different set-ups and objectives. They have been used to analyze the links between the physical workstation conditions and occupant satisfaction, as well as for nonlinear effects between aspects of occupant satisfaction. In general, hierarchical regression is an accepted practice in the behavioural science research community (Kerlinger \& Lee, 2000; Pedhazur, 1997; Tabachnick \& Fidell, 2001).

\subsection{Gaps in Literature and Thesis Contributions}

Having reviewed existing studies and some typical analysis procedures that have been used, there are some gaps in the literature that this thesis addresses. Firstly, studies involving human subjects, particularly in scientifically uncontrolled field environments are extremely complicated in nature. As such, the additional data that this thesis uses, some of which have not been previously analyzed, is of value to the field. There is value in reviewing the consistency of the results across multiple datasets and many years, particularly when many confounding factors are accounted for in the analysis (i.e. multivariate).

Secondly, studies that employ matched data often assume a linear proportional relationship between the IEQ predictor and occupant satisfaction. Yet some researchers (e.g. Martellotta et al. 2016; Kim and de Dear 2012), utilizing questionnaire data, have suggested that several satisfaction measures of components of IEQ have asymmetric (nonlinear) effects on overall satisfaction. However, no current studies have extended this concept into studies using physical measured IEQ parameters. This thesis extends upon the scope of these previous works to incorporate the physical measured parameters in the analysis of nonlinear effects. This can 
lead to better understanding of the relationship between these predictors and occupant satisfaction as well as dissatisfaction.

Additionally, much of the research to-date utilizing physical IEQ measurements use compliance with published standards as a metric as an indication of a high performing indoor environment. Existing field studies rarely examine whether compliance with these standards will result in higher occupant satisfaction. This thesis explicitly examines the effects of compliance with these IEQ standards and whether they have a positive influence on satisfaction in an uncontrolled, real-world setting.

Lastly, the POE literature to-date also largely focuses on the of individual workspace factors, and rarely incorporate aspects beyond the immediate workstation. Although some researchers have considered workstation factors and specific amenities parameters in the same study (Agha-Hossein et al., 2013; Hua et al., 2011), most research to date focuses on one of either individual workstation characteristics or isolated amenities features, but not both. This thesis expands upon what is traditionally incorporated in POEs to incorporate the effects of the broader building amenities on occupants. This further allows for a comparison of the relative importance of aspects of the indoor environment versus the building amenities on occupant satisfaction and productivity indices. 


\section{Methodology}

To examine and answer the individual research questions outlined, the analyses in this thesis are separated into two sections. The first section focuses on the effects of the workstation conditions in relation to research questions $\# 1$ and $\# 2$. The second section extends upon the scope of the first section to incorporate the effects of the building amenities outside of the workstation, in relation to research question \#3. The data used in this thesis are post-occupancy data gathered from real world, scientifically uncontrolled buildings and occupants; this will be discussed in more detail in Section 4.

In this thesis, hierarchical multiple regression was chosen as the analysis method due to the data type and the observations of interest. Multiple linear regressions are best suited for when the outcome (dependent) variable is continuous, such that the effects across the middle (i.e. less extreme) ranges are better represented. Contrasted with regression tools such as logistic regression, multiple linear regression does not 'reduce' the resolution of the satisfaction variables by creating artificial categories (i.e. bins). Furthermore, hierarchical regression allows for a predefined sequence of steps where the effects of individual predictors can be examined independently while their shared variance is considered. This approach allows the researcher to select the predictors and their order of entry based on subject knowledge and the specific hypotheses of interest. Although inter-correlation between predictors can create unexpected effects and mis-partition of variance, tolerance statistics are available as guidance to ensure that excessively inter-correlated predictors are not included in the analyses. Hierarchical regressions are easy to interpret and offer a straightforward approach to isolate and control for the effects of individual predictors within a hierarchy. It also facilitates comparisons with the other prior studies that employ this method in terms of effect size and significance. 
Various hierarchical multiple regression models were used with varying inputs and setups based on the research question of interest. A flowchart summarizing the overall set-up of the different sections in this thesis is shown in Figure 2 below. All statistical analyses in this thesis were performed using IBM SPSS Statistics 24.

Figure 2. Summary overview of thesis set-up

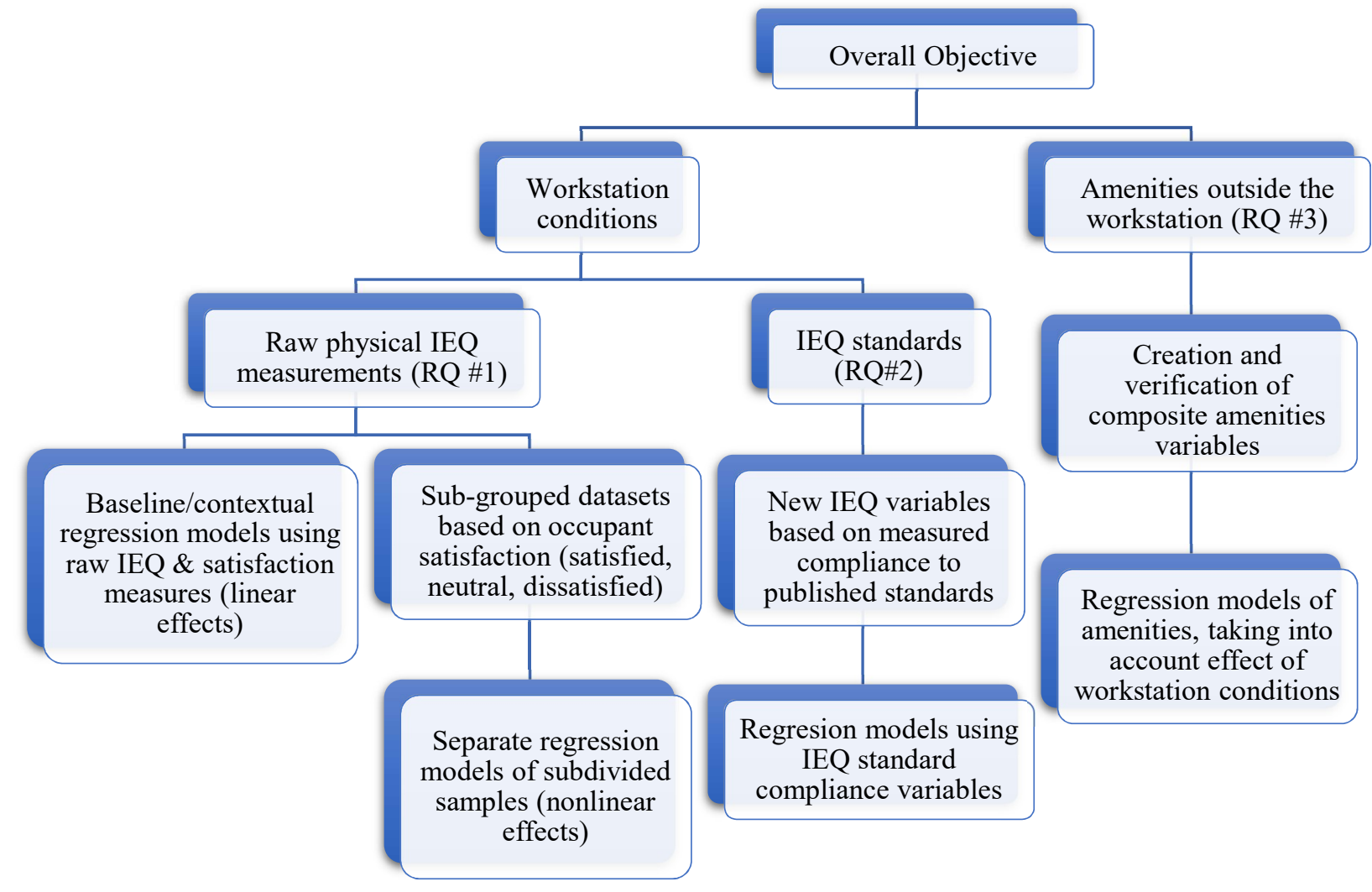

\subsection{Analysis of the Physical Workstation Environment}

This section of the thesis aims to better understand how different aspects of the physical workstation conditions and indoor environment affect occupant satisfaction. Matched occupant survey responses and physical measured data are used in the analyses.

The outcome variables used in this section are measures of satisfaction with individual components of the indoor environment. This included three composite variables created by 
averaging the responses of multiple questions relevant to that aspect of the indoor environment: satisfaction with lighting, satisfaction with ventilation and temperature, and satisfaction with acoustics and privacy. These composite measures are rated on a 7-point Likert type scale, with $1=$ very dissatisfied, $4=$ neutral, and $7=$ very satisfied. For more detailed information on the contents of the subscale composite measures of indoor environment and their formulation, refer to Veitch et al. (2007).

In these regression models, the predictor (independent) variables were input as three separate blocks to isolate the effects of each block on the outcome (dependent) variables. The first block of predictors were the demographic variables, followed by workstation characteristics and then the physical IEQ parameters. This is consistent with what has been done previously by others (e.g. Charles et al., 2006; Veitch et al., 2005).

\subsubsection{Research Question \#1}

To explore the first research question, the relationship between various demographic factors, workstation characteristics, measurements of the indoor environment, and occupant satisfaction are evaluated. The analyses consider both linear relationships as well as the possible asymmetric, 'non-linear' effects of the physical workspace factors, as outlined in Kano's Model of Satisfaction (Kano et al., 1984).

\section{Linear Relationships}

The analyses to evaluate the 'linear' relationships between the variables of interest is performed using hierarchical multiple linear regression. The predictor (i.e. input) variables are demographics (sex and age), workstation characteristics (office enclosure and window proximity), and relevant aspects of physical indoor environment measurements.. 
While this approach and analysis of the linear relationships have been investigated in previous published studies using some of the data in this thesis (Leder et al. 2016; Charles et al. 2006; Veitch et al. 2005), the value of this step lies in the context it provides for the subsequent sections. Not only does this establish 'baseline' results with which the other results can compare with, a consistent group of IEQ predictor variables (or as similar as possible) are retained between the analyses of the two different datasets. This was not done in previous studies and in this study, this approach ensures comparable results between the datasets and adds further value to this procedure. This allows for an evaluation of the consistency of the observations across different groups of occupants, over two decades, in scientifically uncontrolled environments of real buildings. These analyses also take advantage of newly collected data, which contribute to a larger, more homogenous dataset that has not been previously analyzed. This can lead to additional insight and previously unobserved results through the comparison of two datasets with similar analysis procedures.

\section{Asymmetric Relationships}

The asymmetric or 'non-linear' effects of the various IEQ variables were evaluated using an application of Kano's Model of Satisfaction. An approximation of this model is adopted to facilitate the use of physical measured IEQ parameters. Three sub-groupings in the data, consisting of the dissatisfied, neutral, and satisfied respondents, were created to evaluate each aspect of environmental satisfaction. The aim was to capture the entire satisfaction spectrum and approximate the possible asymmetric effects using the three separate linear models. Figure 3 and Figure 4 shown a visualization of this approximation. The rationale is that if a predictor was only statistically significant for the dissatisfied respondent group but not the others, it behaved like a basic factor. The reverse is true for a bonus factor, where the predictor is only statistically 
significant for the satisfied respondent group. To be consistent with the previous section, the predictor variables were entered as three separate blocks.

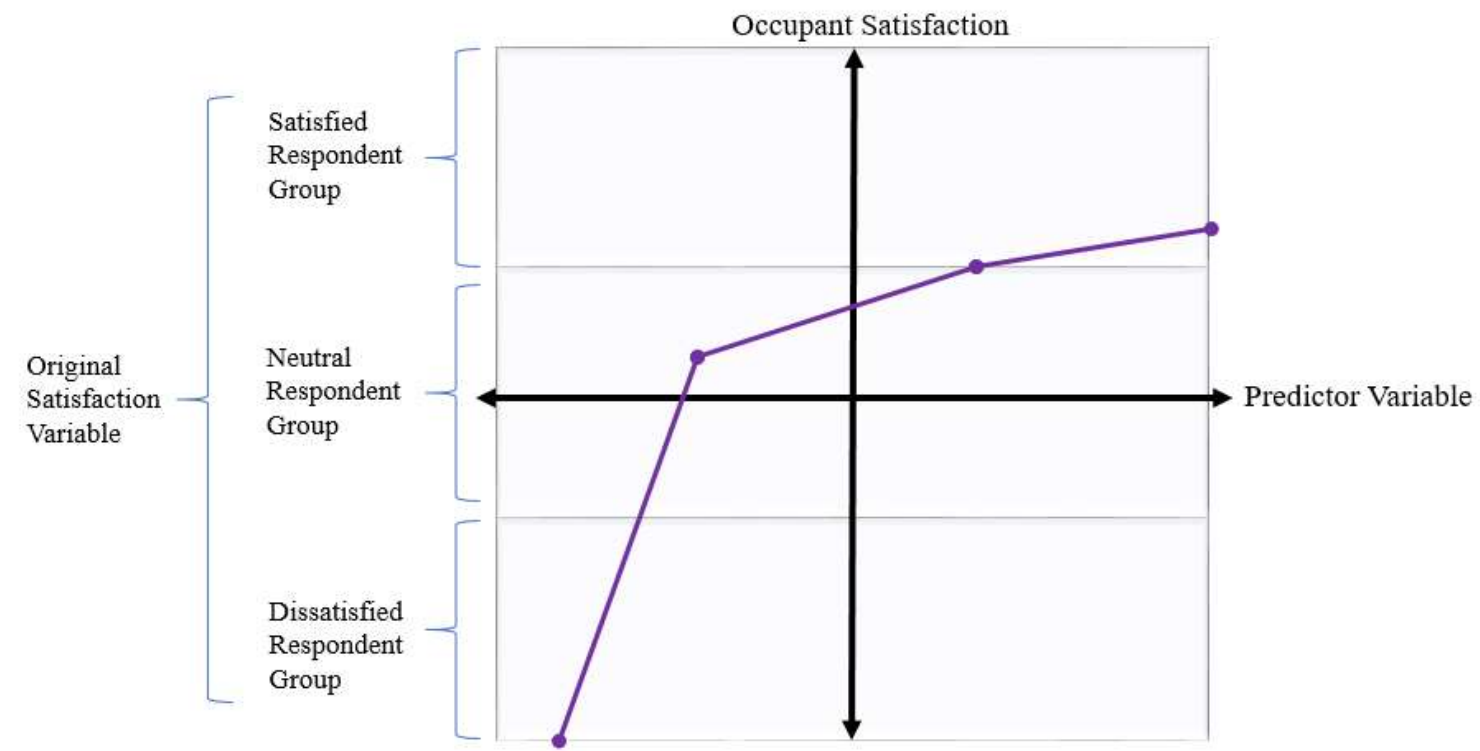

Figure 3. Visualisation of a basic factor using the three-line regression model set-up

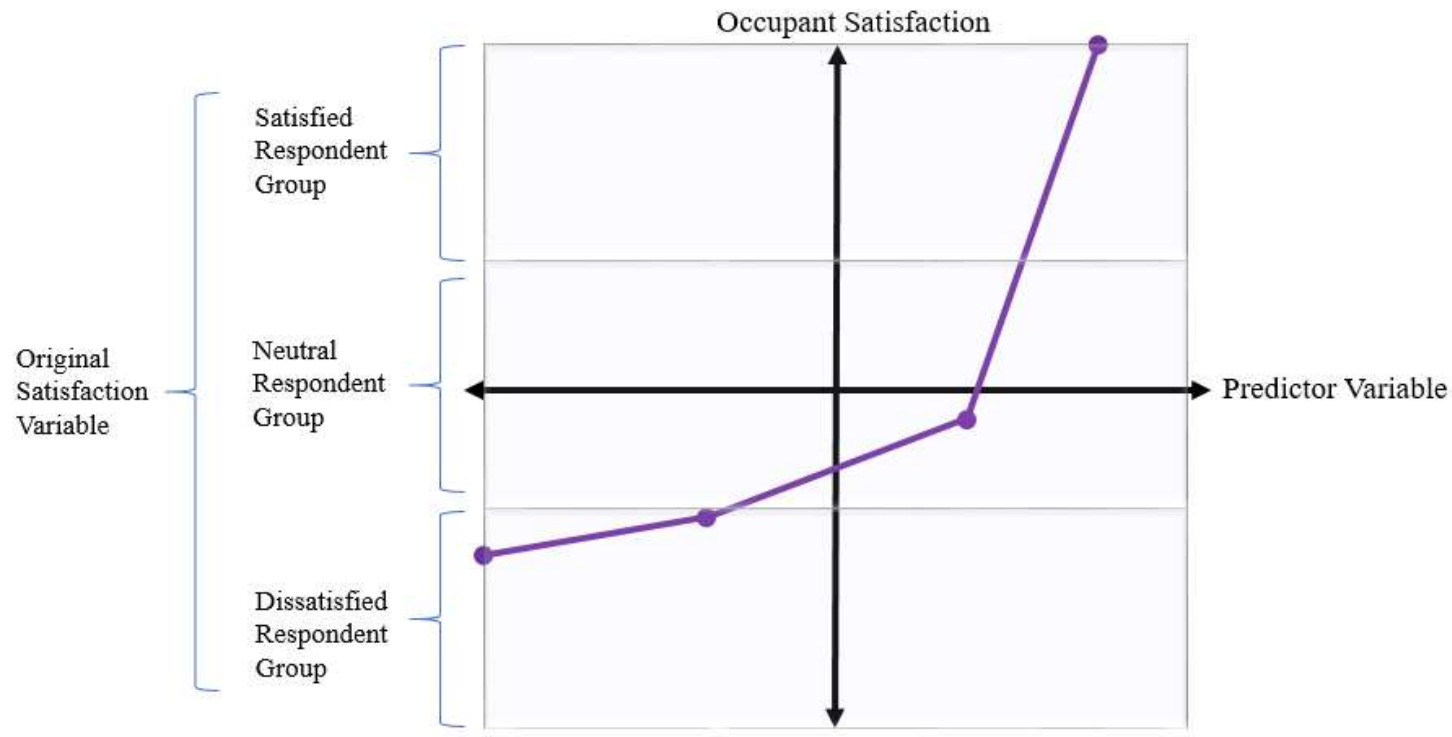

Figure 4. Visualisation of a bonus factor using the three-line regression model set-up 


\subsubsection{Research Question \#2}

In an extension of the first research question, the second research question focuses on the effectiveness of various indoor environment guidelines and references on occupant satisfaction. Within this research question, North American standards and guidelines, to which these buildings would be expected to conform, are examined to evaluate their impact on occupant environmental satisfaction. Three IEQ standards were selected, corresponding to the three aspects of indoor environment previously examined: lighting, acoustics, and the thermal environment. A summary of the standards and their recommended range of values is presented below in Table 1. While there exists other references and guidelines for various regions and governing organizations, it is impractical to evaluate every available standard. The chosen standards were selected to be representative of the current consensus on building performance and are consistent in that they are all overseen by the American National Standards Institute (ANSI).

Table 1 - List of standards/guidelines and their recommended range

Standard/Guideline

ANSI/IESNA RP-1-12 (2012): American National 30 Standard Practice for Office Lighting ANSI/ASA S12.2 (2008): American National Standard Criteria for Evaluating Room Noise

ANSI/ASHRAE Standard 55 (2017): Thermal Environmental Conditions for Human Occupancy

\section{Recommended Range Aspect of IEQ}

Lighting illuminance)

44 to $48 \mathrm{dBA}$

Acoustics (background noise)

$\leq 10 \%$ PPD

Thermal

$-0.5 \leq \mathrm{PMV} \leq+0.5$

The lighting and acoustics reference values are self-explanatory in their interpretation. However, for the calculation of thermal comfort some assumptions were made. For clothing insulation (clo), an average value of 0.5 was assumed for data collected in the summer months, 0.7 for data collected in the fall and spring, and 1.0 for data collected in the winter. A metabolic 
equivalent (MET) value of 1.1 was assumed throughout, which is equivalent to typing at a desk. These assumptions are based on the ASHRAE 55 (2017) standard, which suggests an insulation value of 0.5 clo in the summer and a value of 1.0 clo in the winter, and an average MET of 1.1 in an office setting. Other researchers have frequently used similar assumptions in field studies of buildings (Choi et al., 2010; Liang et al., 2014; MacNaughton et al., 2016; Silva et al., 2017; Tanabe et al., 2015). It is expected that actual occupant clothing levels will vary based on a myriad of factors such as personal preference and workplace expectations. The same applies for metabolic rates, which can differ depending on the activity performed at a given time, body type, and other factors. However, the assumptions made in these analyses are standard assumptions that have been widely used in existing literature and generally accepted in the absence of more detailed occupant information. To provide some context, Table 2 below outlines some typical clothing ensembles and their insulation values for both male and female occupants.

Table 2 - Example clothing ensembles (taken from ASHRAE 2017)

\begin{tabular}{llll}
\hline Sample Woman's Ensemble & Iclo, clo & Sample Man's Ensemble & Iclo, clo \\
\hline Bra & 0.01 & Men's briefs & 0.04 \\
Panties & 0.03 & Shoes & 0.02 \\
Pantyhose/stockings & 0.02 & Calf-length socks & 0.03 \\
Shoes & 0.02 & Short-sleeve dress shirt & 0.19 \\
Short-sleeve dress shirt & 0.19 & Straight trousers (thin) & 0.15 \\
Skirt (knee-length thin) & 0.14 & Net, metal or wooden-side arm chair & 0.00 \\
Net, metal or wooden-side arm chair & 0.00 & & \\
& & & 0.41 \\
Total & 0.41 & Total & 0.45 \\
\hline
\end{tabular}

Again, the predictors were input as three separate blocks to be consistent with previous sections and to isolate the effects of each block on the outcomes. The first and second block of inputs consisted of demographic variables and various workstation characteristics, respectively. 
The last block contained variables reflecting adherence to guideline values in the measured environmental variable of interest (e.g. whether desktop illuminance measurements were between 300-500 lux etc.).

\subsection{Analysis of Amenities Outside of the Immediate Workstation (Research}

\section{Question \#3)}

Amenities, defined as "something that provides comfort, convenience, or enjoyment" (Merriam Webster Online, n.d.) is the subject of the third research question. The aim is to better understand how the elements outside of the physical workstation conditions (i.e. broader building amenities) affect occupant satisfaction and productivity. The effects of the broader amenities are evaluated using questionnaire data, and the outcome measures in the analyses are various occupant satisfaction and organizational productivity metrics. Table 3 outlines the outcome variables used and their sources in literature. 
Table 3 - Outcome variables and their sources in literature

\begin{tabular}{|c|c|c|}
\hline Questionnaire Variable & Description & Source \\
\hline $\begin{array}{l}\text { Overall Environmental } \\
\text { Satisfaction }\end{array}$ & $\begin{array}{l}\text { Two-items related to overall conditions in the } \\
\text { physical environment }\end{array}$ & $\begin{array}{l}\text { Veitch, Charles, Farley, \& } \\
\text { Newsham, } 2007\end{array}$ \\
\hline Job Satisfaction & Single-item measure of overall job satisfaction & $\begin{array}{l}\text { Dolbier, Webster, McCalister, } \\
\text { Mallon, \& Steinhardt, } 2005\end{array}$ \\
\hline Work Engagement & $\begin{array}{l}\text { Nine-item integrated Utrecht Work Engagement } \\
\text { Scale (UWES) }\end{array}$ & $\begin{array}{l}\text { Schaufeli, Bakker, \& Salanova, } \\
2006\end{array}$ \\
\hline $\begin{array}{l}\text { Organizational } \\
\text { Commitment }\end{array}$ & $\begin{array}{l}\text { Six-item scale of affective organizational } \\
\text { commitment }\end{array}$ & Meyer, Allen, \& Smith, 1993 \\
\hline Intent to Turnover & $\begin{array}{l}\text { Three-item scale of employee intent to turnover } \\
\text { (look for another job) }\end{array}$ & Colarelli, 1984 \\
\hline Workplace Image & $\begin{array}{l}\text { Three-item scale on employee opinions } \\
\text { concerning the match between physical work } \\
\text { environment and their understanding of corporate } \\
\text { values }\end{array}$ & Laing, 2005 \\
\hline Visual Discomfort & Short version of visual discomfort scale & Wibom \& Carlsson, 1987 \\
\hline Physical Discomfort & $\begin{array}{l}\text { Adapted from literature and placed in same format } \\
\text { as visual discomfort symptoms }\end{array}$ & Hedge, Erickson, \& Rubin, 1992 \\
\hline
\end{tabular}

Measures such as job satisfaction, organizational commitment, and workplace engagement are not traditionally thought of as measures of organizational productivity. Older industrial production line models of organizational productivity have conventionally been measured in terms of units of output or the performance of a certain activity (e.g. typing words per minute) in a given time. However, there is increasing acceptance that in modern white-collar workplaces, productivity is better represented by multi-metric indicators including parameters like job satisfaction, employee well-being, organizational commitment, and other outcomes (Newsham et al. 2017). This being the case, the terminology of organizational productivity metrics (indices, measures etc.) are used in this thesis to encompass these various outcome variables.

The amenities satisfaction questionnaire items used were drafted specifically for the NCR field study, and there are no previous composite measures developed. The purpose of creating 
the composites is to have a more reliable measure of the concept of an outcome by reducing measurement errors in individual questionnaire items. As such, data reduction from judgement are used to establish new predictor variables for satisfaction with various amenities. Principal components analysis (PCA) was then performed as a secondary check to verify the need for adjustments to the groupings. Cronbach's alpha was then used to evaluate the internal consistency (i.e. reliability) of these measures, and a threshold of 0.7 is widely used in research as an acceptable cut-off (Nunnally, 1978).

This is followed by hierarchical regression (similar to the previous sections), in which amenities predictors are related to the satisfaction and organizational outcome variables. The predictor variables were input as four separate blocks. The first block of inputs consisted of demographic characteristics of the occupants. Next, workstation characteristics were entered as a second block. As the third step, satisfaction with individual aspects of the indoor environment were entered. In the fourth and final step, variables related to satisfaction with amenities were entered. A standard set of predictor variables was retained in each regression model. Using a fixed set of predictors is not necessarily maximally predictive but facilitates interpretation and comparisons between outcomes. 


\section{Input Data}

As previously mentioned, this research thesis utilizes available in-situ field data gathered from real world buildings. The data came from three large building evaluation field studies and was gathered over the last two decades by teams of researchers from NRC. The three field studies are: the cost-effective open-plan environments project (COPE), post-occupancy evaluation of green buildings project (GPOE), and the National Capital Region workplace evaluation project (NCR).

\subsection{COPE Field Study}

The COPE field study involved data collected from public and private sector employees in nine buildings in Canada and the United States. Of the buildings, five were occupied by public sector Canadian organizations, and four were occupied by private sector organizations (in both Canada and the United States). A breakdown of the sample buildings is shown in Table 4 below. The data was collected from 779 workstations and their occupants between Spring 2000 and Spring 2002. Measurements of the physical environment and occupant satisfaction questionnaire responses were collected simultaneously at each individual workstation during a 10-15 minute period. The occupants responded to the survey questionnaire in an adjacent workstation while the researchers conducted physical measurements at their own workstations. 
Table 4 - COPE Study Buildings

\begin{tabular}{llll}
\hline Identification & Sector & Country & City \\
\hline COPE-1 & Private & USA & San Rafael \\
COPE-2 & Private & USA & San Rafael \\
COPE-3 & Private & Canada & Ottawa \\
COPE-4 & Private & USA & San Francisco \\
COPE-5 & Government & Canada & Ottawa \\
COPE-6 & Government & Canada & Ottawa \\
COPE-7 & Government & Canada & Toronto \\
COPE-8 & Government & Canada & Montreal \\
COPE-9 & Government & Canada & Quebec City \\
\hline
\end{tabular}

Physical measurements were taken using a cart-and-chair system, shown in Figure 5.

During a workstation measurement, the occupant's regular chair was removed and replaced with the measurement chair. The measurement chair had sensors to record sound level, temperature and air speed, relative humidity, concentrations of various air pollutants, and illuminance. Two illuminance meters on cables were also placed on the work surface at four fixed locations to measure horizontal desktop illuminance. The researcher also manually recorded various characteristics of the workstation, such as the size, height of surrounding panels, number of enclosed sides of the workstation, presence of a window, luminaire type, presence of task light, location of nearest air supply, and the presence of nearby high-noise areas. Additional acoustic and illuminance measurements were taken at night. The physical measurements at each workstation lasted approximately 10-15 minutes in duration. 


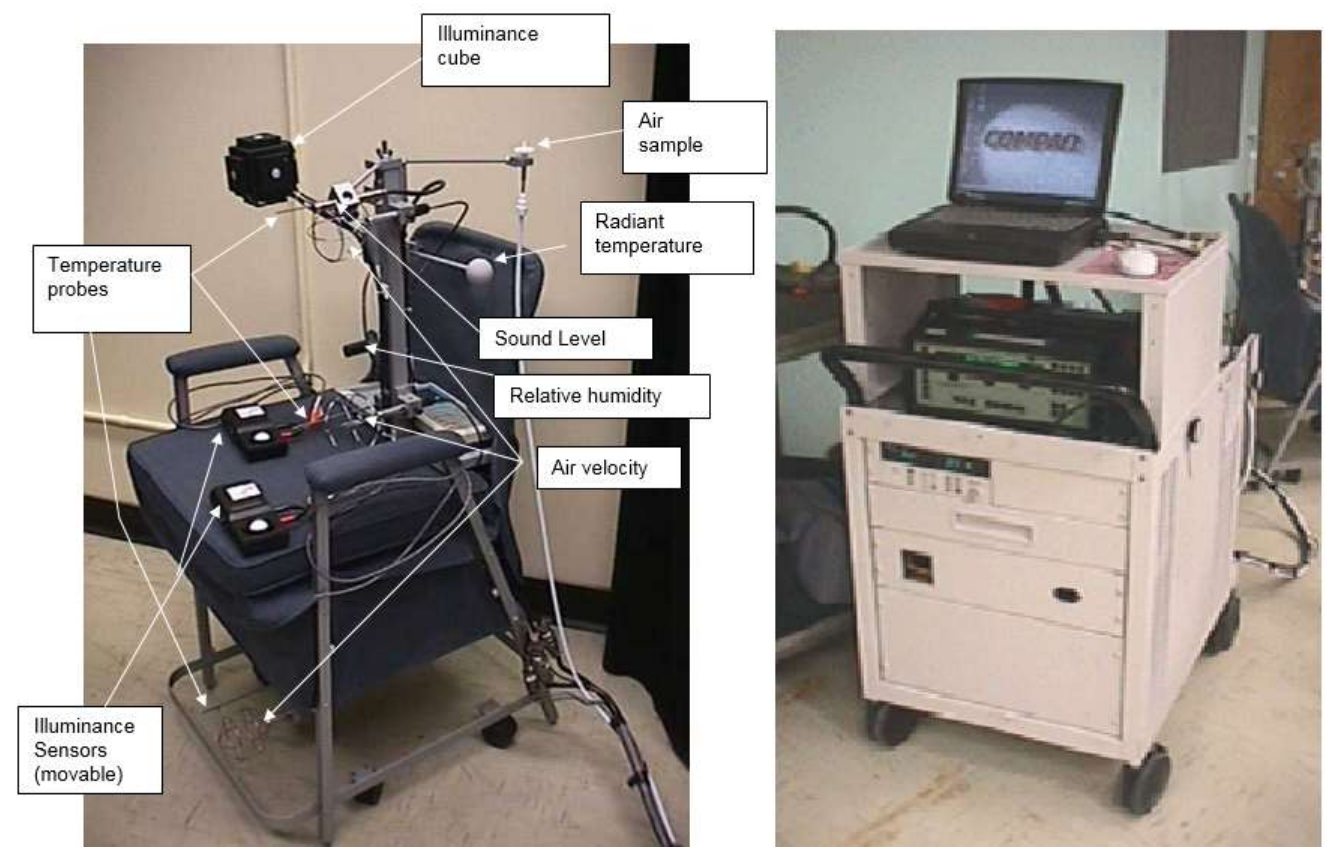

Figure 5. Cart and chair used for physical IEQ measurements in the COPE study (taken from Newsham et al. 2002)

The occupant questionnaire consisted of 27 items, covering satisfaction with individual aspects of the indoor environment at the workstation, the overall environment, and their jobs. Participants were also asked to rank-order the importance of seven physical features, and to provide basic demographic characteristics. The environmental satisfaction questions were rated on a 7-point Likert type scale, from very unsatisfactory to very satisfactory. For more details regarding the data collection methodology, refer to Veitch, Farley, and Newsham (2002).

\subsection{GPOE Field Study}

The GPOE field study involved data collected from public and private sector employees in 24 buildings across Canada and the northern United states. Of these buildings, 12 were conventional buildings and 12 were "green" buildings with some form of green building certification. The data was collected from occupants and representative workspaces in the offices 
between May 2010 and October 2011. Table 5 below outline the buildings in this field study. Unlike the COPE field study, the physical environment measurements of the workspace did not occur at the same time as the occupant responses to the questionnaire. The questionnaires were distributed electronically online to the participants who had a three-week period to respond, while physical measurements were collected at a representative sample of the workspaces by the researchers.

Table 5 - GPOE Study Buildings

\begin{tabular}{lllll}
\hline Identification & Sector & Country & City & Green Certification \\
\hline GPOE-A & Government & Canada & Regina & LEED Silver \\
GPOE-B & Government & Canada & Saskatoon & \\
GPOE-C & Government & Canada & Toronto & \\
GPOE-D & Government & Canada & Toronto & LEED Platinum \\
GPOE-E & Government & Canada & Fredericton & \\
GPOE-F & Private Sector & USA & Boise & LEED Platinum \\
GPOE-G & Private Sector & USA & Boise & \\
GPOE-H & Government & USA & Idaho Falls & LEED Gold \\
GPOE-I & Government & USA & Idaho Falls & \\
GPOE-J & Non-Profit & Canada & Ottawa & LEED Gold \\
GPOE-K & University & Canada & Ottawa & \\
GPOE-LNP & University & Canada & Vancouver & \\
GPOE-MOQ & University & Canada & Vancouver & Various \\
GPOE-R & Government & Canada & Ottawa & \\
GPOE-S & Government & Canada & Ottawa & LEED Platinum \\
GPOE-T & Private Sector & USA & Holland & LEED Gold \\
GPOE-U & Private Sector & USA & Grand Rapids & \\
GPOE-V & Private Sector & USA & Grand Rapids & \\
GPOE-W & Government & Canada & Ottawa & \\
GPOE-X & Government & Canada & Ottawa & Go Green Plus \\
\hline
\end{tabular}


Physical data was collected using the NICE Cart (NRC Indoor Climate Evaluator), shown in Figure 6. The NICE Cart performs a similar function to the measurement chairs used in the COPE field study, providing a detailed snapshot of the indoor environment over a 10-15 minute period. A schematic of the layout of the NICE cart during typical measurement locations is shown in Figure 7. In general, the physical environment measurement process did not change significantly between the GPOE and COPE field studies. However, some sensors measuring the same parameters were updated/changed and several parameters were measured in this study that weren't collected in the COPE study. These differences are discussed in more detail in Section 4.4.1. Physical data were collected at a total of 977 representative workstations across the 24 buildings.
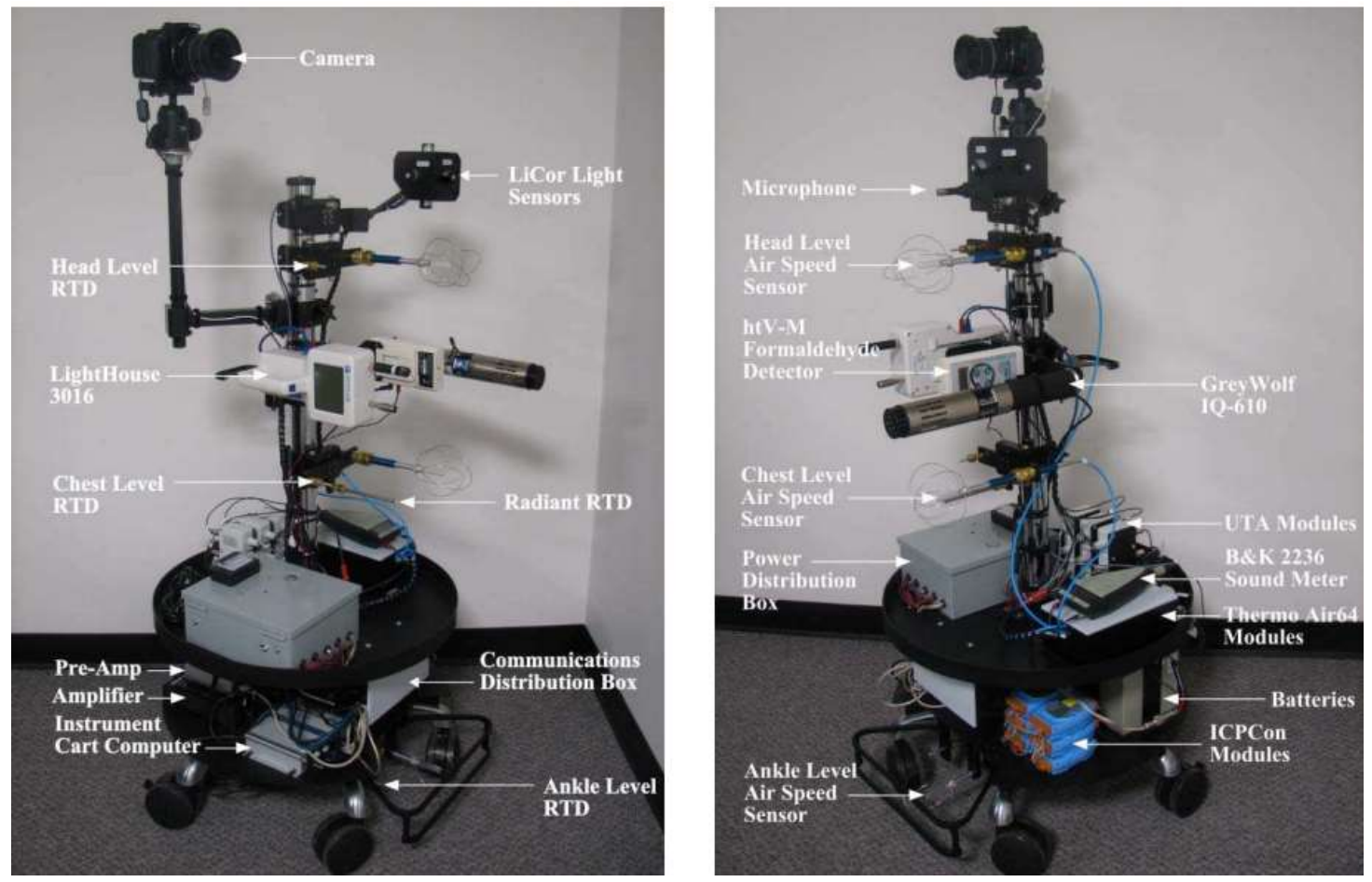

Figure 6. NICE Cart used for physical IEQ measurements in the GPOE and NCR studies (taken from Newsham et al. 2012) 


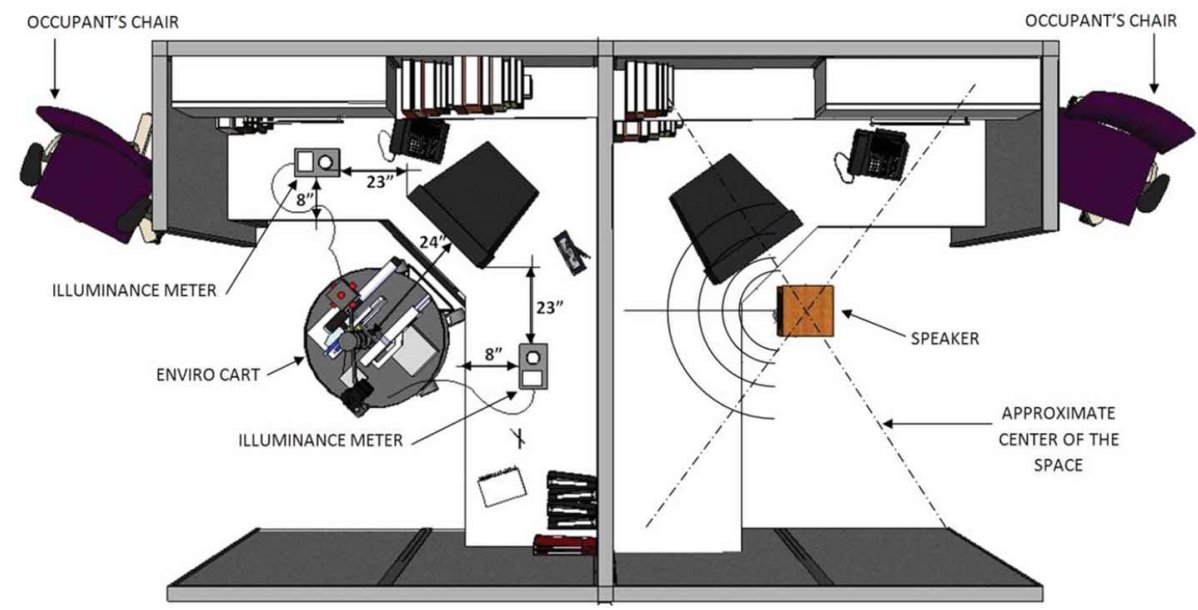

Figure 7. In-situ schematic diagram of NICE Cart (taken from Newsham et al. 2012)

The occupant questionnaire used in the GPOE field study significantly expanded upon the one used in the COPE study. A total of 148 questionnaire items was split into seven modules: one core module and six other modules. All respondents were asked to complete the core module, and then randomly assigned two of the six other modules to reduce the time burden on respondents. The core questions were very similar to the contents of the COPE questionnaire that covered environmental satisfaction, job satisfaction, and demographics. The only changes being additional questions regarding years in the workforce/organization, and the job satisfaction variable changing from a two-item factor in COPE to a one-item factor in GPOE. The other modules consisted largely of items validated in prior studies, and include items on organizational commitment, workplace image, internal communications, acoustics, thermal comfort, chronotype, sleep quality, positive/negative feelings (affect), health, commuting, and environmental attitudes. A summary of the various questionnaire modules is shown below in Table 6. A total of 2,545 occupant questionnaire responses were gathered from across the 24 buildings in this study. For more details regarding the data collection methodology, refer to Newsham et al. (2013). 
Table 6 - Summary of questionnaire modules in GPOE field study

\begin{tabular}{ccl}
\hline Module & \# of Questions & Description \\
\hline Core & 35 & $\begin{array}{l}\text { Environmental and job satisfaction, demographics, job demands } \\
1\end{array}$ \\
16 & $\begin{array}{l}\text { Organizational commitment, workplace image, internal } \\
\text { communications }\end{array}$ \\
2 & 11 & Acoustics \\
3 & 34 & Thermal comfort \\
4 & 13 & Chronotype, sleep quality, affect balance \\
5 & 25 & Commuting, attitude regarding environment \\
6 & &
\end{tabular}

\subsection{NCR Field Study}

\subsubsection{Setting}

The NCR field study involved data collected from a large public-sector building located in the National Capital Region in Canada. The building has not undergone major renovation since its construction in the 1970 s. Several amenities and functional features are available to the occupants. Many local bus stops are located near the site, and approximately 1,700 parking spaces are available for a monthly fee. There are also 16 shower facilities and nearly 300 bike racks available for occupant use. Exterior green space includes a small landscaped park and a courtyard area adjacent to the building. Many scenic trails are also near the building site.

A food court is available within the building, providing access to food options. The building is also connected to a commercial mall, which hosts a variety of services including restaurants, hair salons, dental offices, banks, postal stations and more.

As this study took place within a single large office building, some amenities such as parking and transit access are common to all occupants. Other amenity features like local 
meeting rooms, printers, and kitchen access differed amongst respondents. The commercial shopping concourse also has many different options, which will appeal in varying ways to different people.

\subsubsection{Occupant Survey and Physical Measurements}

The data was collected from occupants and representative workspaces in the building between October and November of 2017. The data collection methodology in the NCR study is nearly identical to that of the GPOE field study.

Physical measurements were conducted by the researchers at a representative sample of the workstations using the NICE Cart. The physical parameters recorded in this field study are the same as those in the GPOE study, using the same sensor models. Physical measurements were conducted using the NICE Cart at a total of 265 workstations in the building. Occupant questionnaires were distributed electronically to the respondents. Like the GPOE field study, the

physical environment measurements of the workspace did not occur concurrently with the occupant questionnaire responses.

5,269 occupants in the building were invited to complete an online questionnaire across a three-week period during October and November of 2017. The questionnaire itself had a total of 201 items and was separated into four modules: one core module and three other modules. Each respondent in this study was asked to complete a core module of the questionnaire and was then randomly assigned one of three additional modules. Respondents were given the option to complete more than one additional module if they wished. A summary of the various questionnaire modules is shown below in Table 7. Much of the contents of the NCR occupant questionnaire were the same as the one used in the GPOE field study. However, there were several notable differences. For instance, items related to satisfaction with various local 
amenities were incorporated into the NCR questionnaire, as this was an area of interest for this POE study. The more detailed questions used in the GPOE field study regarding thermal comfort and noise were also excluded in this questionnaire. A total of 1,953 responses were received (response rate of $37 \%)$.

Table 7- Summary of questionnaire modules in the NCR field study

\begin{tabular}{ccl}
\hline Module & \# of Questions & Description \\
\hline Core & 76 & $\begin{array}{l}\text { Environmental and job satisfaction, demographics, job } \\
\text { demands, workplace location, convenience and features }\end{array}$ \\
1 & 49 & $\begin{array}{l}\text { Organizational commitment, workplace image, internal } \\
\text { communications, engagement, interpersonal relationships, } \\
\text { transportation } \\
\text { Comfort-related modifications/complaints, sustainability } \\
\text { awareness, satisfaction with local amenities, break/lunch } \\
\text { activities } \\
\text { Chronotype, sleep quality, mood, health }\end{array}$ \\
3 & 42 &
\end{tabular}

\subsection{Dataset Summary}

\subsubsection{Physical Workstation Environment}

In the analyses of the physical workstation environment, data from all three field studies are used. However, prior to performing any analysis of the data, there must be considerations of the validity in combining all three individual datasets. While the data from the three field studies captured similar information, there were several differences in the collection methodology and the age of the data where combining all three datasets is deemed inappropriate.

Firstly, the participants of the COPE field study completed the questionnaire as the same time as when the researchers performed the physical measurements (i.e. simultaneously); this was not the case for the GPOE and NCR field studies. In the latter studies collection of the physical and survey data did not happen concurrently, as the occupants completed a web-based 
questionnaire at their leisure across a span of multiple weeks. Although the IEQ measurements are assumed to be representative of overall conditions across all three studies, these differences in measurement and questionnaire response conditions can be a source of error across the datasets. Additionally, the COPE field study used a different set of sensors than the GPOE and NCR field studies (refer to Table 8). While the equipment was calibrated to achieve accurate readings, this is a possible difference that can influence the results across the datasets. Furthermore, the COPE field study took place 15 years prior to the NCR study, and there can be differences regarding occupant expectations of the workspace and their perceptions of comfort across this timespan. The differences (or similarities) in the effects of physical environment parameters in relation to occupant satisfaction across the timespan of more than a decade is an area of interest within this thesis and will be explored. For these reasons, it was deemed inappropriate to combine the COPE dataset with the more recent GPOE and NCR field studies. 
Table 8 - Instrumentation used for physical data capture

\begin{tabular}{|c|c|c|c|c|}
\hline $\begin{array}{l}\text { Measured IEQ } \\
\text { Factor }\end{array}$ & Equipment Model & Range & Accuracy & $\begin{array}{l}\text { Field Study } \\
\text { Used }\end{array}$ \\
\hline \multirow{2}{*}{ Illuminance } & Minolta T-1 & 0.01 to 99900 lux & $\pm 7 \%$ & COPE \\
\hline & LiCor LI-210 & 0 to 60000 lux & $\pm 5 \%$ & GPOE \& NCR \\
\hline \multirow[b]{2}{*}{ Air Velocity } & TSI- 8475 & 0 to $1 \mathrm{~m} / \mathrm{s}$ & $\pm 3 \%$ & COPE \\
\hline & ThermoAir 6/64 & 0 to $1 \mathrm{~m} / \mathrm{s}$ & $\begin{array}{l} \pm 1.5 \% \text { reading value } \\
\pm 0.5 \% \text { of full scale }\end{array}$ & GPOE \& NCR \\
\hline \multirow[t]{2}{*}{ Sound Level } & Rion NA-29 & $\begin{array}{l}27 \text { to } 130 \mathrm{~dB}(\mathrm{a}) \\
31.5 \mathrm{~Hz} \text { to } 8 \mathrm{kHz}\end{array}$ & $\pm 0.1 \mathrm{~dB}$ & COPE \\
\hline & B\&K 2236 & 18 to $140 \mathrm{~dB}$ & Type $1(\sim \pm 0.7 \mathrm{~dB})$ & GPOE \& NCR \\
\hline \multirow{2}{*}{ Temperature } & Omega RTD & Room temp & $<0.1^{\circ} \mathrm{C}$ & COPE \\
\hline & GrayWolf IQ 610 & $-10^{\circ}$ to $+70^{\circ} \mathrm{C}$ & $\pm 0.3^{\circ} \mathrm{C}$ & GPOE \& NCR \\
\hline \multirow[b]{2}{*}{ Relative Humidity } & General Eastern RH2 & 20 to $95 \%$ & $\pm 2 \%$ & COPE \\
\hline & GrayWolf IQ 610 & 0 to $100 \% \mathrm{RH}$ & $\begin{array}{l} \pm 2 \% \mathrm{RH}<80 \% \mathrm{RH} \\
( \pm 3 \% \mathrm{RH}>80 \% \mathrm{RH})\end{array}$ & GPOE \& NCR \\
\hline \multirow{2}{*}{ Pollutants (VOCs) } & B\&K 1302 & & $\pm 0.3 \mathrm{ppm}(\mathrm{TVOC})$ & COPE \\
\hline & GrayWolf IQ 610 & 5 to $20000 \mathrm{ppb}$ & & GPOE \& NCR \\
\hline Carbon dioxide & GrayWolf IQ 610 & 0 to $10000 \mathrm{ppm}$ & $\pm 3 \%$ reading $\pm 50 \mathrm{ppm}$ & GPOE \& NCR \\
\hline Carbon monoxide & GrayWolf IQ 610 & 0 to $500 \mathrm{ppm}$ & $\begin{array}{l} \pm 2 \mathrm{ppm}<50 \mathrm{ppm} \\
\pm 3 \% \text { reading }>50 \mathrm{ppm}\end{array}$ & GPOE \& NCR \\
\hline Formaldehyde & Htv-M & 0 to $10 \mathrm{ppm}$ & $\pm 25 \%$ & GPOE \& NCR \\
\hline $\begin{array}{l}\text { Pollutants } \\
\text { (Particulates) }\end{array}$ & LightHouse 3016 & 0.3 to $10.0 \mu \mathrm{m}$ & $10 \%(20 \%$ for $0.3 \mu \mathrm{m})$ & GPOE \& NCR \\
\hline
\end{tabular}

The GPOE field study incorporated data from both green and conventional buildings.

Previous researcher have consistently demonstrated the benefits of green buildings on occupant satisfaction, even when subject to similar indoor environment conditions (e.g. MacNaughton et al. 2016; Holmgren, Kabanshi, and Sörqvist 2017; Pei et al. 2015). A prior study by Leder et al. (2016) using the GPOE dataset has also illustrated that all else being equal, occupants in green buildings were significantly more satisfied with their workstation environment. As such, the differences in occupant perception in "green" versus conventional buildings is another inconsistency in the datasets and can present an additional source of error in the analyses. This thesis does not focus on the benefits of green buildings on occupant satisfaction.

Based on the key differences outlined above, it was determined that blindly combining the entirety of the three datasets was not appropriate. The matched questionnaire and physical 
measurement samples from the conventional buildings within the GPOE dataset will be combined with the NCR dataset, as they had identical data collection methodologies. The matched COPE dataset will be analyzed separately using the same analysis methods. The data were assessed for extreme outliers or clear equipment malfunctions. Many of the statistical procedures used in this thesis assume normality in the data, all predictor and outcome variables were tested for normality. The criteria used were skewness values between +3 and -3 and kurtosis values between +8 and -8 , to maintain consistency with previously published studies (Kline, 1997). Some samples in the dataset were excluded in the analyses based on the above criteria.

Table 9 outlines the demographic characteristics of the two final data groupings used in the physical workstation environment portion of this thesis. There were some notable differences in the sample demographics of the two data groups. For instance, a larger percentage of the respondents in the conventional GPOE + NCR sample were female, where the COPE sample was more even in terms of the sexes. The COPE sample also had a larger percentage of technical staff, though the job type categories were open to interpretation by the respondents. Nonetheless, a varied sample was obtained among the demographic categories in each data group. 
Table 9 - Demographics characteristics of the two data groups

\begin{tabular}{|c|c|c|c|c|c|c|c|}
\hline \multicolumn{2}{|l|}{ Age } & \multicolumn{2}{|l|}{ Sex } & \multicolumn{2}{|l|}{ Job Category } & \multicolumn{2}{|l|}{ Education } \\
\hline \multicolumn{8}{|c|}{ COPE Sample $(N=772)$} \\
\hline $18-29$ & $13 \%$ & Female & $48 \%$ & Administrative & $27 \%$ & High School & $12 \%$ \\
\hline $20-39$ & $33 \%$ & Male & $52 \%$ & Technical & $25 \%$ & Community College & $15 \%$ \\
\hline $40-49$ & $34 \%$ & & & Professional & $38 \%$ & University Courses & $15 \%$ \\
\hline $50-59$ & $18 \%$ & & & Managerial & $9 \%$ & Undergraduate Degree & $35 \%$ \\
\hline$>60$ & $3 \%$ & & & & & Graduate Degree & $23 \%$ \\
\hline \multicolumn{8}{|c|}{ Conventional GPOE + NCR Sample $(N=194)$} \\
\hline $18-29$ & $8 \%$ & Female & $66 \%$ & Administrative & $28 \%$ & High School & $12 \%$ \\
\hline $20-39$ & $22 \%$ & Male & $34 \%$ & Technical & $7 \%$ & Community College & $16 \%$ \\
\hline $40-49$ & $29 \%$ & & & Professional & $47 \%$ & University Courses & $9 \%$ \\
\hline $50-59$ & $35 \%$ & & & Managerial & $18 \%$ & Undergraduate Degree & $35 \%$ \\
\hline$>60$ & $6 \%$ & & & & & Graduate Degree & $28 \%$ \\
\hline
\end{tabular}

\subsubsection{Broader Amenities Outside of the Immediate Workstation}

The analyses of the broader amenities outside of the workstation utilizes questionnaire data from the NCR study. As stated in previous sections, the NCR field study extended upon what was incorporated in previous building surveys and includes items regarding building and site amenities. The focus of this section of the thesis is on the effect of satisfaction with the building amenities evaluated via survey data. The physical indoor workstation environment measurements were not incorporated, as it was not the emphasis of this part of the research. The physical data was also a substantially smaller sample $(\mathrm{N}=265$ without survey data; $\mathrm{N}=82$ with matched survey data), which would greatly limit the strength of the statistical analyses.

Like the analyses of the physical work environment, all the predictor and outcome variables were tested for normality. Again, skewness values between +3 and -3 and kurtosis 
values between +8 and -8 were used. Table 10 outlines the demographic characteristics of the questionnaire respondents in the NCR study.

Table 10 - Demographics characteristics of the NCR respondents $(\mathrm{N}=1953)$

\begin{tabular}{llllllll}
\hline Age & \multicolumn{3}{c}{ Sex } & & Job Category & & Education \\
\hline $18-29$ & $11.4 \%$ & Male & $57.5 \%$ & Administrative & $28.9 \%$ & Highschool & $14.1 \%$ \\
$30-39$ & $22.5 \%$ & Female & $42.2 \%$ & Technical & $10.3 \%$ & Community College & $22.7 \%$ \\
$40-49$ & $32.1 \%$ & Other & $0.3 \%$ & Professional & $41.1 \%$ & University Courses & $7.6 \%$ \\
$50-59$ & $28.4 \%$ & & & Managerial & $19.8 \%$ & Undergraduate Degree & $39.3 \%$ \\
$>60$ & $5.6 \%$ & & & & & Graduate Degree & $16.3 \%$ \\
\hline
\end{tabular}




\section{Physical Workstation Conditions - Results}

This section presents the results regarding the physical workstation conditions, organized according to the aspect of IEQ (lighting, acoustics, thermal). Within each aspect of IEQ, research questions \#1 and \#2 are explored.

As previously mentioned, hierarchical multiple regressions were used to analyze the data, and the results can be interpreted as follows. The columns under each $\beta$ heading show the standardized regression coefficient (or slope coefficient) for a predictor variable. For every standard deviation increase in the predictor variable, $\mathrm{x}$, there will be $\beta^{*} \mathrm{x}$ standard deviation increase in the outcome variable; larger regression coefficients represent larger correlations between the predictor variable and the outcome within that analysis. The asterisks present in the tables represent $p$-values (significance levels), with thresholds at $0.05,0.01$, and $0.001 . P$-values less than the significance level thresholds represent a statistically significant result. Tolerance represents the redundancy of a predictor variable in the overall analysis; the smaller the value, the more redundant its contribution to the regression. Effect sizes $\left(\mathrm{R}^{2}\right)$ are interpreted using the small (1\%), medium (9\%), and large (25\%) effect size criteria from Cohen (1988).

\subsection{Descriptive Statistics and Variable Set-up}

Prior to jumping straight into the statistical analyses, it is important to set the context for the data that is used. The descriptive statistics (means, medians, standard deviations etc.) for the predictor and outcome variables were generated for both datasets. Table 11 below outlines the descriptive statistics for the occupant environmental satisfaction measures of both matched survey-measurement datasets used in this section. Of the individual aspects of environmental satisfaction, occupants were most satisfied with the lighting across both data groups, with a median score of 5, corresponding to "satisfied". In comparison, satisfaction with acoustics \& 
privacy had medians of 3.9 and 4.3, and satisfaction with ventilation and temperature had medians of 4.3 and 4.0 (corresponding roughly to "slightly satisfied").

Table 11 - Descriptive statistics of IEQ satisfaction measures (matched samples)

\begin{tabular}{|c|c|c|c|c|c|c|}
\hline Variable & Mean & Median & Std. Dev. & Min. & Max. & $\mathbf{N}$ \\
\hline \multicolumn{7}{|l|}{ (a) COPE Sample } \\
\hline $\begin{array}{l}\text { Satisfaction with lighting (average of } 5 \\
\text { questions) }\end{array}$ & 4.8 & 5.0 & 1.2 & 1.4 & 7.0 & 769 \\
\hline $\begin{array}{l}\text { Satisfaction with acoustics and privacy } \\
\text { (average of } 10 \text { questions) }\end{array}$ & 3.9 & 3.9 & 1.1 & 1.0 & 6.7 & 768 \\
\hline $\begin{array}{l}\text { Satisfaction with ventilation and } \\
\text { temperature (average of } 3 \text { questions) }\end{array}$ & 4.3 & 4.3 & 1.4 & 1.0 & 7.0 & 768 \\
\hline
\end{tabular}

(b) Conventional GPOE + NCR Sample

Satisfaction with lighting (average of 5 questions)

$\begin{array}{llllll}4.9 & 5.0 & 1.1 & 1.0 & 7.0 & 191\end{array}$

Satisfaction with acoustics and privacy (average of 10 questions)

$\begin{array}{llllll}4.2 & 4.3 & 1.3 & 1.0 & 7.0 & 194\end{array}$

Satisfaction with ventilation and temperature (average of 3 questions)

$\begin{array}{lllll}3.9 & 4.0 & 1.5 & 1.0 & 7.0\end{array}$

194

Note: Satisfaction measures on a 7-point scale, $1=$ very unsatisfied, $4=$ neutral, $7=$ very satisfied.

Table 12 below outlines the descriptive statistics of the raw physical IEQ parameters used in this section of the thesis. The measured physical workstation conditions are generally comparable between the two datasets, although there are some notable differences. For instance, the GPOE+NCR sample generally had lower background sound levels and speech intelligibility, likely due to the inclusion of enclosed private workstations within this data sample. The associated bivariate correlations of the raw predictor variables are shown in Appendix B. 
Table 12 - Descriptive statistics of physical measured parameters (matched samples)

\begin{tabular}{|c|c|c|c|c|c|c|}
\hline Variable & Mean & Median & Std. Dev. & Min. & Max. & $\mathbf{N}$ \\
\hline \multicolumn{7}{|l|}{ (a) COPE Sample } \\
\hline Partition Height (inches) & 57.5 & 63.0 & 13.8 & 30.0 & 104.0 & 772 \\
\hline Desktop Illuminance (lux) & 451 & 401 & 255 & 4 & 1957 & 772 \\
\hline Sound Level (dBA) & 46.4 & 46.7 & 3.8 & 36.2 & 59.9 & 730 \\
\hline Speech Intelligibility Index (SII) & 0.5 & 0.5 & 0.1 & 0.1 & 0.9 & 725 \\
\hline Radiant Temperature $\left({ }^{\circ} \mathrm{C}\right)$ & 23.4 & 23.4 & 0.8 & 20.6 & 26.5 & 772 \\
\hline Air Temperature $\left({ }^{\circ} \mathrm{C}\right)$ & 23.3 & 23.3 & 0.9 & 20.4 & 28.7 & 772 \\
\hline Relative Humidity (\%) & 29.9 & 28.7 & 10.7 & 13.1 & 58.8 & 772 \\
\hline Air Velocity (m/s) & 0.10 & 0.08 & 0.05 & 0.01 & 0.43 & 772 \\
\hline \multicolumn{7}{|c|}{ (b) Conventional GPOE + NCR Sample } \\
\hline Desktop Illuminance (lux) & 487 & 406 & 276 & 58 & 1631 & 194 \\
\hline Sound Level (dBA) & 42.1 & 42.1 & 3.8 & 33.4 & 52.7 & 194 \\
\hline Speech Intelligibility Index (SII) & 0.34 & 0.33 & 0.17 & 0.04 & 0.87 & 194 \\
\hline Radiant Temperature $\left({ }^{\circ} \mathrm{C}\right)$ & 22.9 & 23 & 1.2 & 19.1 & 27.6 & 194 \\
\hline Air Temperature $\left({ }^{\circ} \mathrm{C}\right)$ & 22.9 & 22.9 & 1 & 18.9 & 27.4 & 194 \\
\hline Relative Humidity (\%) & 27.6 & 26 & 8.3 & 15.4 & 53.6 & 194 \\
\hline Air Velocity (m/s) & 0.12 & 0.11 & 0.05 & 0.02 & 0.37 & 194 \\
\hline Particulates, $2.5 \mu \mathrm{m}\left(\mu \mathrm{g} / \mathrm{m}^{3}\right)$ & 2.9 & 1.2 & 5.2 & 0.3 & 26.8 & 194 \\
\hline
\end{tabular}

The tables below outline the coding and setup of the predictor and outcome variables used in the various analyses of the physical workstation. The coding of the raw predictor variables used in this section is shown in Table 13. Window location is divided into workstations with and without windows, and the windows in the sampled buildings were generally not operable. Workstation enclosure in the COPE sample is examined using minimum partition height, as the sampled buildings only contained open-plan offices. The workstation enclosure in the conventional GPOE + NCR sample is separated into private workstations (full height walls and a door) and open workstations (all other configurations). 
Table 13 - Summary of the raw predictor variables (matched samples)

\begin{tabular}{|c|c|c|c|}
\hline \multicolumn{2}{|c|}{ COPE Sample } & \multicolumn{2}{|c|}{ Conventional GPOE + NCR Sample } \\
\hline Variable & Description & Variable & Description \\
\hline \multirow[t]{5}{*}{ Age } & $18-29(\mathrm{~N}=100)$ & Age & $18-29(\mathrm{~N}=15)$ \\
\hline & $20-39(\mathrm{~N}=247)$ & & $20-39(\mathrm{~N}=42)$ \\
\hline & $40-49(\mathrm{~N}=255)$ & & $40-49(\mathrm{~N}=55)$ \\
\hline & $50-59(\mathrm{~N}=137)$ & & $50-59(\mathrm{~N}=67)$ \\
\hline & $>60(\mathrm{~N}=20)$ & & $>60(\mathrm{~N}=12)$ \\
\hline \multirow[t]{2}{*}{ Sex } & $0=$ Female $(\mathrm{N}=368)$ & Sex & $0=$ Female $(\mathrm{N}=126)$ \\
\hline & $1=$ Male $(\mathrm{N}=397)$ & & $1=$ Male $(\mathrm{N}=65)$ \\
\hline \multirow[t]{2}{*}{ Window } & $\begin{array}{l}0=\text { No window in workstation } \\
(\mathrm{N}=461)\end{array}$ & Window & $\begin{array}{l}0=\text { No window in workstation } \\
(\mathrm{N}=108)\end{array}$ \\
\hline & $\begin{array}{l}1=\text { Window in workstation } \\
(\mathrm{N}=311)\end{array}$ & & $1=$ Window in workstation $(\mathrm{N}=84)$ \\
\hline $\mathrm{MinPH}$ & $\begin{array}{l}\text { Minimum workstation partition } \\
\text { height (inches) }\end{array}$ & WSEnclosure & $\begin{array}{l}0=\text { Open workstation }(\mathrm{N}=138) \\
1=\text { Private workstation }(\mathrm{N}=56)\end{array}$ \\
\hline AveIlluDesk & \\
\hline $\mathrm{AW}$ & \multicolumn{3}{|c|}{ A-weighted background sound level (dBA) } \\
\hline SII & \multicolumn{3}{|c|}{$\begin{array}{l}\text { Speech intelligibility index ( } 0 \text { to } 1 ; 0=\text { completely unintelligible and } 1=\text { completely } \\
\text { intelligible) }\end{array}$} \\
\hline AirTemp & \multicolumn{3}{|c|}{ Air temperature measured at head level $\left({ }^{\circ} \mathrm{C}\right)$} \\
\hline RH & \multicolumn{3}{|l|}{ Relative humidity (\%) } \\
\hline AirVelocity & \multicolumn{3}{|c|}{ Air velocity measured at head level $(\mathrm{m} / \mathrm{s})$} \\
\hline
\end{tabular}

Table 14 below summarizes the sub-groupings of the dissatisfied, neutral, and satisfied occupants used to evaluate asymmetric relationships in the application of Kano's Model. The conventional GPOE + NCR sample generally had a small number of cases for people dissatisfied with lighting and acoustics. As such, the dissatisfied respondents were combined with the neutral respondents for this sample (Light_DisNeu and Acou_DisNeu in the table below). 
Table 14 - Summary of sub-grouped satisfaction variables (matched samples)

\begin{tabular}{|c|c|c|c|c|}
\hline $\begin{array}{l}\text { Original } \\
\text { Variable }\end{array}$ & $\begin{array}{l}\text { Sub- } \\
\text { Grouping }\end{array}$ & Description & COPE & $\begin{array}{r}\text { Conventional } \\
\text { GPOE + NCR }\end{array}$ \\
\hline \multirow{4}{*}{$\begin{array}{l}\text { Satisfaction } \\
\text { with } \\
\text { Lighting } \\
\text { (SAT_L) }\end{array}$} & Light_Dissat & Respondents dissatisfied with lighting $(<3)$ & 87 & $*$ \\
\hline & Light_Neutral & Respondents neutral with lighting (3-5) & 278 & * \\
\hline & Light_DisNeu & Respondents dissatisfied or neutral with lighting $(<5)$ & * & 86 \\
\hline & Light_Sat & Respondents satisfied with lighting $(>5)$ & 392 & 104 \\
\hline \multirow{4}{*}{$\begin{array}{l}\text { Satisfaction } \\
\text { with } \\
\text { Acoustics \& } \\
\text { Privacy } \\
\text { (SAT_AP) }\end{array}$} & Acou_Dissat & $\begin{array}{l}\text { Respondents dissatisfied with acoustics \& privacy } \\
(<3)\end{array}$ & 168 & $*$ \\
\hline & Acou_Neutral & Respondents neutral with acoustics \& privacy (3-5) & 409 & $*$ \\
\hline & Acou_DisNeu & $\begin{array}{l}\text { Respondents dissatisfied or neutral with acoustics \& } \\
\text { privacy }(<5)\end{array}$ & * & 127 \\
\hline & Acou_Sat & Respondents satisfied with acoustics \& privacy $(>5)$ & 133 & 64 \\
\hline $\begin{array}{l}\text { Satisfaction } \\
\text { with }\end{array}$ & Temp_Dissat & $\begin{array}{l}\text { Respondents dissatisfied with ventilation \& } \\
\text { temperature }(<3)\end{array}$ & 186 & 60 \\
\hline $\begin{array}{l}\text { Ventilation } \\
\&\end{array}$ & Temp_Neutral & $\begin{array}{l}\text { Respondents neutral with ventilation \& temperature } \\
(3-5)\end{array}$ & 262 & 77 \\
\hline $\begin{array}{l}\text { Temperature } \\
\text { (SAT_VT) }\end{array}$ & Temp_Sat & $\begin{array}{l}\text { Respondents satisfied with ventilation \& temperature } \\
(>5)\end{array}$ & 308 & 54 \\
\hline
\end{tabular}

Note: * in the table indicates groupings that are not applicable for the given dataset. Satisfaction measures on a 7 -point scale, $1=$ very unsatisfied, $4=$ neutral, $7=$ very satisfied.

Table 15 summarizes the re-coded 'dummy' physical IEQ reference variables, representing compliance with the IEQ standards. In the conventional GPOE + NCR data sample, very few cases had sound levels greater than $48 \mathrm{dBA}$, and a separate category for such values was not large enough for statistical validity. Based on the nature of the outcomes of interest, the acoustics limits for this sample was adjusted to whether measured background noise levels were below recommendations. There were also very few cases where the measured conditions were warmer than the thermal comfort criteria in both datasets. As such, the thermal environment criteria were adjusted to whether the measured conditions were below (colder) the ASHRAE 55 thermal comfort zone. 
Table 15 - Categorization of IEQ reference variables (matched samples)

\begin{tabular}{|c|c|c|c|}
\hline Variable & Description & COPE & $\begin{array}{r}\text { Conventional } \\
\text { GPOE + NCR } \\
\end{array}$ \\
\hline \multirow[t]{2}{*}{ IllumBelow } & $0=$ Greater than 300 lux & 558 & 149 \\
\hline & $1=$ Less than 300 lux & 214 & 45 \\
\hline \multirow[t]{2}{*}{ IllumRef } & $0=$ Outside of $300-500$ lux & 478 & 109 \\
\hline & $1=$ Within $300-500 \operatorname{lux}$ & 294 & 85 \\
\hline \multirow[t]{2}{*}{ IllumAbove } & $0=$ Less than 500 lux & 508 & 130 \\
\hline & $1=$ Greater than 500 lux & 264 & 64 \\
\hline \multirow[t]{2}{*}{ SoundBelow } & $0=$ Greater than $44 \mathrm{dBA}$ & 525 & 59 \\
\hline & $1=$ Less than $44 \mathrm{dBA}$ & 205 & 135 \\
\hline \multirow[t]{2}{*}{ SoundRef } & $0=$ Outside of $44-48 \mathrm{dBA}$ & 451 & * \\
\hline & $1=$ Within $44-48 \mathrm{dBA}$ & 279 & $*$ \\
\hline \multirow[t]{2}{*}{ SoundAbove } & $0=$ Less than $48 \mathrm{dBA}$ & 484 & * \\
\hline & $1=$ Greater than $48 \mathrm{dBA}$ & 246 & $*$ \\
\hline \multirow[t]{2}{*}{ ThermBelow } & $0=$ Not colder than thermal comfort zone & 497 & 84 \\
\hline & $1=$ Colder than thermal comfort zone & 275 & 110 \\
\hline
\end{tabular}

Note: $*$ in the table indicates groupings that are not applicable for the given dataset.

\subsection{Lighting}

The results pertaining to the lighting conditions in the occupants' workstation are presented in this section. Prior to performing any statistical analyses of the data, it is often beneficial to have a visualization of the data in question. Graphs of lighting satisfaction versus measured desktop illuminance were generated to observe any obvious trends or patterns in the data. Figure 8 below show the graphs for both datasets. The plotted data was very scattered, and no obvious trends or patterns emerged. While it is possible that the measured lighting has little effect on satisfaction, it could also be due to confounding factors within the data which render a simple $\mathrm{X}$ vs $\mathrm{Y}$ graph inadequate for observing these relationships. As such, the use of various multi-variate statistical procedures throughout this thesis are necessary to effectively extract the various relationships between the workstation parameters. 


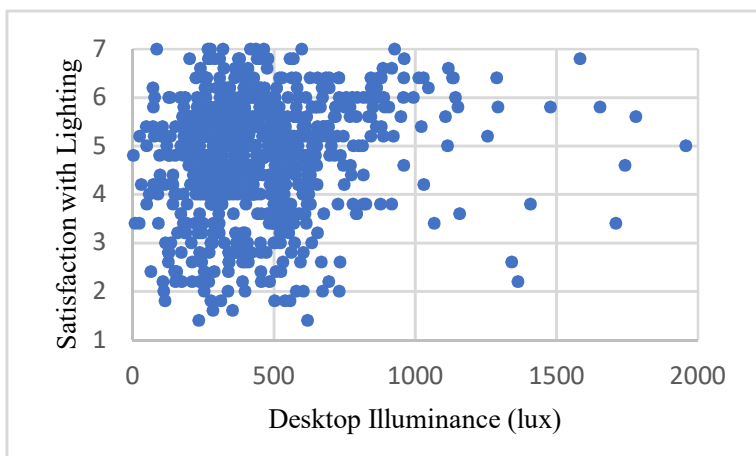

(a) COPE Sample

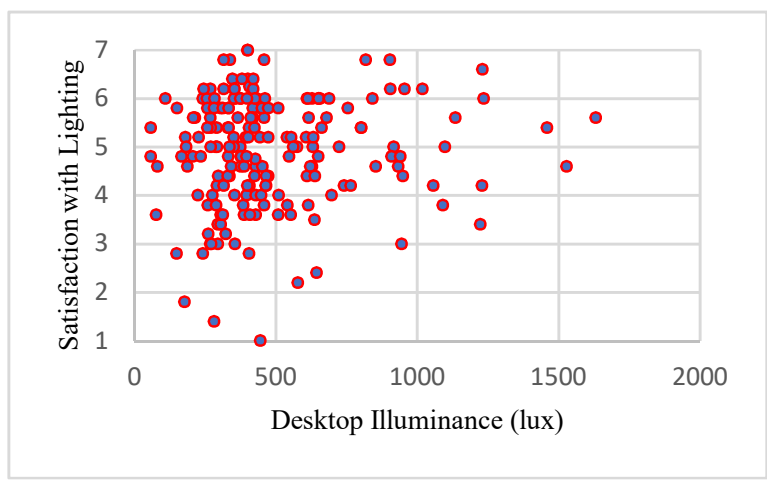

(b) Conventional GPOE + NCR Sample

Figure 8. Satisfaction with lighting vs. desktop illuminance

Table 16 summarizes results for satisfaction with lighting using unaltered physical predictors and satisfaction measures. In both data samples, the demographic factors were not shown to have a significant influence on satisfaction with lighting.

The results across both data samples show that workstations with windows was a statistically significant predictor of lighting satisfaction. This benefit persisted in the regression model even after the inclusion of desktop illuminance as a predictor. This result suggests that that even after considering the raw measured illuminance levels, window proximity had a positive effect on satisfaction. This is a logical and expected observation. It suggests that lighting satisfaction is not only about the provided light levels, but also the presence of natural light and awareness of the exterior. Window offices receive more natural lighting from the exterior while also providing views of the outside, which allow occupants to be better connected with nature. The benefits of natural daylighting and access to outside views have been well-documented in existing literature (Aries et al., 2010; Elzeyadi, 2011; Farley \& Veitch, 2001; Frontczak et al., 2012; Leder et al., 2016). Existing literature also identify windows as the largest source of glare in an office environment (Aries et al., 2010). It is interesting to note that the possible negative 
influences and drawbacks of windows on glare and subsequent lighting dissatisfaction were not observed in the results.

Table 16 - Satisfaction with lighting regression results

(a) COPE Sample $(N=757)$

\begin{tabular}{lcccc}
\hline & $\boldsymbol{\beta}$ & $\boldsymbol{\beta}$ & $\boldsymbol{\beta}$ & Tolerance \\
\hline Age & 0.03 & -0.02 & -0.02 & 0.94 \\
Sex & 0.02 & 0.01 & 0.01 & 1.00 \\
Window & & $0.27^{* * *}$ & $0.24^{* * *}$ & 0.83 \\
MinPH & & -0.04 & -0.03 & 0.94 \\
AveIlluDesk & & & $0.08^{*}$ & 0.87 \\
\hline $\mathrm{R}^{2}$ Change & 0.00 & $0.07^{* * *}$ & $0.01^{*}$ & \\
Total R & & $0.07^{* * *}$ & $0.07^{* * *}$ & \\
Adjusted $\mathrm{R}^{2}$ & 0.00 & $0.06^{* * *}$ & $0.07^{* * *}$ & \\
\hline
\end{tabular}

(b) Conventional GPOE + NCR Sample (N=190)

\begin{tabular}{lcccc}
\hline & $\boldsymbol{\beta}$ & $\boldsymbol{\beta}$ & $\boldsymbol{\beta}$ & Tolerance \\
\hline Age & 0.00 & -0.04 & -0.04 & 0.96 \\
Sex & 0.12 & 0.12 & 0.12 & 0.99 \\
Window & & $0.29^{* * *}$ & $0.29^{* * *}$ & 0.88 \\
WSEnclosure & & -0.03 & -0.03 & 0.97 \\
AveIlluDesk & & & 0.00 & 0.91 \\
\hline $\mathrm{R}^{2}$ Change & 0.01 & $0.08^{* * *}$ & 0.00 & \\
Total R & & $0.10^{* * *}$ & $0.10^{* *}$ & \\
Adjusted $\mathrm{R}^{2}$ & 0.01 & $0.08^{* * *}$ & $0.07^{* *}$ & \\
\hline
\end{tabular}

Note: Outcome variable is satisfaction with lighting. Tolerance values are shown for variables at the final step. ${ }^{*} \mathrm{p} \leq 0.05,{ }^{* *} \mathrm{p} \leq 0.01,{ }^{* * *} \mathrm{p} \leq 0.001$.

Within the COPE data sample, higher desktop illuminance had an effect on occupant lighting satisfaction even after taking into account the benefits of windows; this effect was not observed within the conventional GPOE + NCR sample. However, the observed effect size $\left(\mathrm{R}^{2}\right)$ were very small, suggesting only marginal benefits to increased lighting. Possible reasons for this observation could be the changes in perceptions/preferences over the years, as well as the evolving workplace set-ups/equipment and the typical types of tasks being performed. When the 
data was collected for the COPE sample (nearly 20 years ago), CRT monitors were the norm, and a larger portion of office-related tasks were paper-based as opposed to electronically. This emphasis in paper-based tasks likely resulted in more intensive use of the desktop and likely required more lighting. In this setting the beneficial effects of a more well-lit space (i.e. more desktop illuminance) could be more influential. In the newer GPOE + NCR sample, flat screen monitors were common in the workplace, along with a shift to more computer-based tasks in the typical white-collar office environment. Paper-based tasks were less common, and it is possible that the benefits of higher desktop illuminance are diminished. Excessively high illuminance levels can also have a negative influence on satisfaction, where there is increased susceptibility to glare on the computer screens during computer-based tasks. Computer monitors also inherently provide back lighting for the occupant to view the screen, and high levels of illuminance are not necessarily required to perform computer-based work. This difference in lighting preference between paper-based versus computer-based tasks have been shown in literature, with occupants performing computer-based tasks preferring dimmer environments (Choi et al., 2012). This will be explored further in the analysis for research question \#2, where the benefits of a specific range of desktop illuminance (i.e. there is an upper threshold of illuminance) is discussed.

In extending the analysis of lighting to the non-linear trends in the data, Table 17 summarizes the sub-grouped lighting regression results. Within the dissatisfied and neutral respondent groups of the COPE sample, there were no observed statistically significant predictors of lighting satisfaction and very little variance is explained in the regression model. Meanwhile, the model of the satisfied group showed both window proximity and workstation partition height to have some influence. This result suggests that window proximity and partition 
height behave more like bonus factors and have more of a positive effect in the satisfied range. However, in comparing these observations with the linear lighting regression model (Table 16), the results suggest that these factors still had benefits across the entire satisfaction spectrum. It is possible that the beneficial effects of windows in the dissatisfied and neutral ranges are less pronounced, so that when analyzed separately with a smaller sample size it was not found to be statistically significant. The observation that lower partition heights increase lighting satisfaction in already satisfied occupants makes logical sense. Interior workstations (i.e. not perimeter workstation) with lower partition walls can receive more lighting from the outside through windows, as well as additional lighting from ceiling fixtures over adjacent spaces. Again, the effect sizes of even the most predictive model (satisfied occupants in COPE) were only medium $\left(\mathrm{R}^{2}=10 \%\right)$, showing the complexities in the data and that the predictor variables did not explain a large part of an occupant's lighting satisfaction.

We previously found a significant, linear, relationship between the linear lighting regression model (all cases) for the COPE dataset. Yet, its positive effects were not observed in the sub-grouped asymmetric analyses; desktop illuminance was not a significant predictor in any of the three sub-grouped COPE models. While there is a relationship between illuminance and lighting satisfaction across the entire satisfaction spectrum (Table 16), it is not particularly large or pronounced. By sub-grouping the satisfaction measures there were less valid cases in each regression model, which could have contributed to the observed statistical insignificance of illuminance. The results appear to support this line of reasoning, as the slope coefficient, $\beta$, and effect size $\left(\mathrm{R}^{2}\right)$ were not particularly large in the overall linear model. This suggests that the effect of illuminance on increasing satisfaction was not particularly large, even across the entire satisfaction range. 
Table 17 - Sub-grouped (asymmetric) lighting regression results

\begin{tabular}{|c|c|c|c|c|c|c|c|c|c|}
\hline \multirow[t]{2}{*}{ (a) } & \multicolumn{3}{|c|}{$\begin{array}{c}C O P E \text { - Light_Dissat } \\
(N=87)\end{array}$} & \multicolumn{3}{|c|}{$\begin{array}{c}\text { COPE - Light_Neutral } \\
(N=27 \overline{8})\end{array}$} & \multicolumn{3}{|c|}{ COPE - Light_Sat $(N=392)$} \\
\hline & $\beta$ & $\boldsymbol{\beta}$ & $\boldsymbol{\beta}$ & $\boldsymbol{\beta}$ & $\boldsymbol{\beta}$ & $\beta$ & $\beta$ & $\beta$ & $\boldsymbol{\beta}$ \\
\hline Age & 0.11 & 0.12 & 0.12 & -0.01 & -0.01 & 0.00 & $0.12 *$ & 0.08 & 0.08 \\
\hline Sex & 0.12 & 0.13 & 0.13 & -0.03 & -0.03 & -0.03 & 0.00 & 0.00 & 0.00 \\
\hline Window & & -0.05 & -0.02 & & -0.03 & -0.03 & & $0.29 * * *$ & $0.28 * * *$ \\
\hline $\mathrm{MinPH}$ & & 0.01 & 0.00 & & 0.03 & 0.03 & & $-0.14 * *$ & $-0.14 * *$ \\
\hline AveIlluDesk & & & -0.06 & & & -0.02 & & & 0.03 \\
\hline $\mathrm{R}^{2}$ Change & 0.03 & 0.00 & 0.00 & 0.00 & 0.00 & 0.00 & 0.01 & $0.09 * * *$ & 0.00 \\
\hline Total $\mathrm{R}^{2}$ & 0.03 & 0.03 & 0.03 & 0.00 & 0.00 & 0.00 & 0.01 & $0.10 * * *$ & $0.10 * * *$ \\
\hline Adjusted $\mathrm{R}^{2}$ & 0.00 & -0.02 & -0.03 & -0.01 & -0.01 & -0.02 & 0.01 & $0.09 * * *$ & $0.09 * * *$ \\
\hline \multirow[t]{2}{*}{ (b) } & & & & \multicolumn{3}{|c|}{$\begin{array}{c}G P O E+N C R- \\
\text { Light_DisNeu }(N=86)\end{array}$} & \multicolumn{3}{|c|}{$\begin{array}{c}G P O E+N C R-L i g h t \_S a t \\
(N=104)\end{array}$} \\
\hline & & & & $\beta$ & $\beta$ & $\beta$ & $\beta$ & $\beta$ & $\beta$ \\
\hline Age & & & & 0.00 & -0.03 & -0.03 & 0.01 & 0.01 & 0.01 \\
\hline Sex & & & & $0.22 *$ & $0.25^{*}$ & $0.25^{*}$ & 0.10 & 0.09 & 0.09 \\
\hline Window & & & & & 0.19 & 0.16 & & 0.11 & 0.11 \\
\hline WSEnclosure & & & & & -0.05 & -0.05 & & -0.07 & -0.07 \\
\hline AveIlluDesk & & & & & & 0.09 & & & 0.00 \\
\hline $\mathrm{R}^{2}$ Change & & & & 0.05 & 0.04 & 0.01 & 0.01 & 0.02 & 0.00 \\
\hline Total $\mathrm{R}^{2}$ & & & & 0.05 & 0.08 & 0.09 & 0.01 & 0.03 & 0.03 \\
\hline Adjusted $\mathrm{R}^{2}$ & & & & 0.03 & 0.04 & 0.03 & -0.01 & -0.01 & -0.02 \\
\hline
\end{tabular}

Note: Outcome variable is satisfaction with lighting. ${ }^{*} p \leq 0.05, * * p \leq 0.01, * * * p \leq 0.001$.

As previously stated, in the analysis of non-linear trends the dissatisfied and neutral respondents were grouped together for the GPOE + NCR dataset. The sub-grouped regression results for this data sample, like that of the COPE sample, also showed little in the way of significant predictors. The results for the dissatisfied-neutral group suggest that males were less likely to be dissatisfied than their female counterparts. This effect was not observed in the satisfied group, which suggests that sex behaved like a basic factor. This result is in line with the findings of other studies, which have shown females to be more prone to visual discomfort (Aries et al., 2010; Thayer et al., 2010). However, in observing the effect sizes of the models, the 
changes in variance explained was not significant. This suggests that while sex could be an influential factor on lighting dissatisfaction, its effects are minimal.

The benefits of windows in the prior analyses were also not observed in sub-grouped GPOE $+\mathrm{NCR}$ sample. As with the discussion of the COPE, it is possible that the beneficial effects of windows were affected by the reduced sample size. Evidence of this was noted in the raw regression results (not captured in the tables shown), where the $p$-value of the window variable was 0.086 . While this result did not meet the significance limit thresholds, it does suggest the possibility that with a larger sample akin to that of the overall linear regression model (Table 16), window proximity might have been a statistically significant predictor. Workstation enclosure was not found to be a statistically significant predictor of lighting satisfaction in the satisfied sub-group results of the GPOE+NCR sample, as partition height was for the COPE sample. This could be a result of taller partitions walls in an open plan office (as is in the COPE sample) blocking out additional lighting from adjacent spaces and windows. In the case of the GPOE+NCR sample where enclosure was separated into private enclosed offices and open offices, this effect may not have been captured in the regression model. In the case of private offices with full height walls, they are typically provided with their individual light fixtures. The walls further prevent the infiltration of light from adjacent offices.

The lack of significant predictors in the lighting analysis of non-linear effects across both data groups prompts a discussion as to some possible reasons. It is possible that there are minimal relationships between the incorporated predictor variables and lighting satisfaction, and that by including other, potentially more telling predictors, the results may change. However, the overall regression results (Table 16) consistently found factors such as window proximity to have an effect, so there are likely also other explanations for these observations. One such 
explanation could be that these results are the artefact of the reduced sample sizes, which can diminish the trends in the data. Compounding on top of this, the respondents of these studies were generally satisfied with lighting, with a median of 5.0 above the neutral point of the satisfaction scale. As such, the dissatisfied and neutral groups had fewer cases than the satisfied group. Evidence of this possibility can be seen in the COPE sample, where the expected significance of window proximity was observed in the larger, satisfied occupant sub-grouping.

The regression results with respect to compliance with the IESNA RP-1-12 (2012) lighting standard as outlined in research question \#2 are shown below in Table 18. The results shown in the first two blocks of this regression table are identical to that of the overall lighting regression model (Table 16), as the sample and inputs for demographics and workstation factors were identical. These factors were left in the regression model to be consistent with previous sections and maintain control of the confounding variables. In the third block of the model IllumBelow and IllumAbove were input as predictors, representing desktop illuminance below, and above the recommended 300-500 lux range, respectively.

Within both datasets, occupants in offices with windows were found to have greater lighting satisfaction, even after the inclusion of the IESNA reference variable. This finding is similar to the results found in the previous regression models. The results for both data groupings also show that the reference variable, IllumBelow, was had a statistically significant effect on lighting satisfaction. This observation indicates that occupants whose measured desktop illuminance were within the IESNA reference range (i.e. 300-500 lux) reported higher levels of lighting satisfaction compared to occupants whose measured illuminance was below the reference criteria, even after taking into account other factors like window proximity. This finding reinforces the validity of the limit thresholds as specified in IESNA RP-1-12 (2012). 
Table 18 - Satisfaction with lighting regression results for IEQ guidelines

(a) COPE Sample $(N=757)$

\begin{tabular}{lcccc}
\hline & $\boldsymbol{\beta}$ & $\boldsymbol{\beta}$ & $\boldsymbol{\beta}$ & Tolerance \\
\hline Age & 0.03 & -0.02 & -0.02 & 0.94 \\
Sex & 0.02 & 0.01 & 0.02 & 0.99 \\
Window & & $0.27 * * *$ & $0.26 * * *$ & 0.89 \\
MinPH & & -0.04 & -0.04 & 0.93 \\
IllumBelow & & & $-0.08^{*}$ & 0.78 \\
IllumAbove & & & -0.02 & 0.77 \\
\hline $\mathrm{R}^{2}$ Change & 0.00 & $0.07 * * *$ & 0.01 & \\
Total R & 0.00 & $0.07 * * *$ & $0.07 * * *$ & \\
Adjusted $\mathrm{R}^{2}$ & 0.00 & $0.06 * * *$ & $0.07 * * *$ & \\
\hline
\end{tabular}

(b) Conventional GPOE + NCR Sample $(N=190)$

\begin{tabular}{lcccc}
\hline & $\boldsymbol{\beta}$ & $\boldsymbol{\beta}$ & $\boldsymbol{\beta}$ & Tolerance \\
\hline Age & 0.00 & -0.04 & -0.02 & 0.95 \\
Sex & 0.12 & 0.12 & 0.10 & 0.97 \\
Window & & $0.29 * * *$ & $0.31^{* * *}$ & 0.90 \\
WSEnclosure & & -0.03 & -0.05 & 0.96 \\
IllumBelow & & & $-0.16^{*}$ & 0.82 \\
IllumAbove & & & -0.13 & 0.77 \\
\hline $\mathrm{R}^{2}$ Change & 0.01 & $0.08^{* * *}$ & 0.03 & \\
Total R & 0.01 & $0.10^{* * *}$ & $0.12^{* * *}$ & \\
Adjusted $\mathrm{R}^{2}$ & 0.00 & $0.08^{* * *}$ & $0.09 * * *$ & \\
\hline Not: Outtcom & & &
\end{tabular}

Note: Outcome variable is satisfaction with lighting. Tolerance values are shown for variables at the final step. ${ }^{*} \mathrm{p} \leq 0.05, * * \mathrm{p} \leq 0.01, * * * \mathrm{p} \leq 0.001$

The results further suggest that having higher desktop illuminance has less bearing on lighting satisfaction than meeting the minimum illuminance threshold of 300 lux. Compared to the linear regression of the raw desktop illuminance variable in Table 16, this result suggests that there is an upper threshold beyond which higher illuminance does not result in more satisfied occupants. This could be due to a myriad of reasons, including more glare at higher illuminances and reduced opportunities for personal adjustments/preferences. Occupants likely each have their own personal preferences and satisfying the minimum lighting level without over-illuminating can have benefits in terms of personal adjustments. In a dimly lit space (that meets minimum lux 
requirements), occupants who prefer more subdued environments are satisfied, and occupants who prefer more well-lit environments can compensate with the use of individual task lights. Contrast this with the scenario of an exceedingly brightly-lit environment, where occupants who prefer more dim environments cannot make personal adjustments.

It was observed in the various lighting regressions above (Tables 16-18) that the effect sizes $\left(\mathrm{R}^{2}\right)$ of the overall models were generally small to medium, with the largest explained variance noted at $12 \%$. While these models were not very predictive, other POE studies utilizing multiple regressions have often found comparable effect sizes in their lighting analysis. For instance, Leder et al., (2016) reported $\mathrm{R}^{2}$ values of 0.11 and 0.21 in their models while an older study by Veitch et al. (2005) found $\mathrm{R}^{2}$ values of 0.07 to 0.11 . This highlights the complexities in this field and the randomness in data obtained from human subjects in an uncontrolled environment. While the results do not show that the input variables are the sole contributors to lighting satisfaction, various statistically significant effects were observed in the models, suggesting that they have incremental benefits.

The results generally showed the workstation characteristics (i.e. windows) to have a larger effect size than that of the physical illuminance measurements, suggesting that window proximity has a larger effect in improving lighting satisfaction in occupants than actual office illuminance. This suggests rather than the increases in illuminance values associated with windows, the beneficial effects of natural light, outside view, and the connection with nature were more important. Existing studies of daylighting and windows in office spaces have supported this idea, and have found that natural and artificial lighting are not perceived the same way by occupants (Elzeyadi, 2011; Galasiu \& Veitch, 2006). While it is impractical in a commercial office environment for every occupant to be seated at the perimeter and have access 
to a window, it does suggest to designers the importance of windows and daylighting. This further reinforces the need to better optimize glazing design/placement and office space planning.

Although the observed effect sizes were generally smaller for measured illuminance predictors, the results still generally showed that occupants preferred more well-lit environments. A possible reason for the small explained variance could be the fact that the occupants were already largely satisfied with their lighting conditions (means of 4.8 and 4.9), and that there were very little correlations to explain in terms of subsequent incremental improvements. This could be an indication that lighting in modern office spaces are generally well-implemented and designed, and so fluctuations in the range of typical office lighting levels did not play a large role in occupant satisfaction. Furthermore, by considering window proximity separately in the analyses, variance that might otherwise have been attributed to desktop illuminance may have been re-allocated.

\subsection{Acoustics and Privacy}

This section of the thesis presents the results pertaining to the acoustic conditions in the occupants' workstation. Like the results of the lighting conditions, visualizations of the data were generated prior to performing any statistical analyses of the data. Graphs of satisfaction with acoustics \& privacy versus measured background noise and speech intelligibility were generated to observe any obvious trends or patterns in the data. Figures 9 and 10 below show the graphs for both datasets. Similar to the graphs of lighting satisfaction, the plotted data were very scattered and no obvious trends or patterns emerged. 


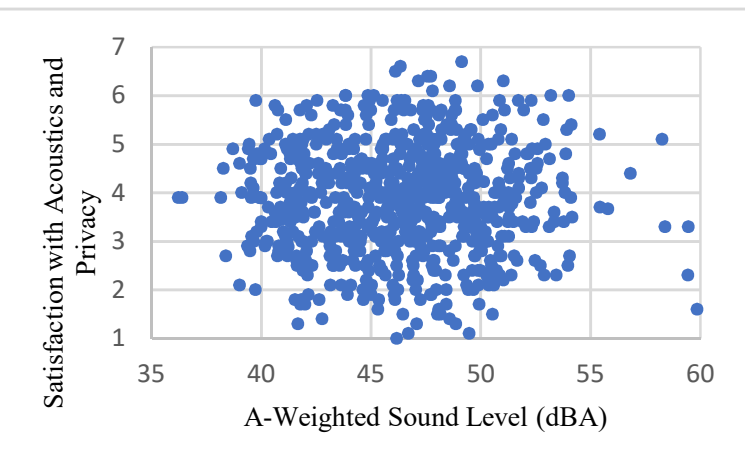

(a) COPE Sample

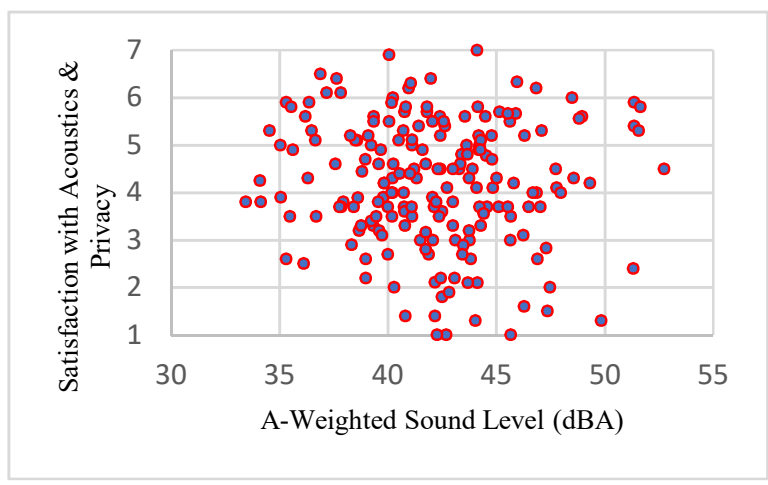

(b) Conventional GPOE + NCR Sample

Figure 9. Satisfaction with acoustics and privacy vs. background sound level

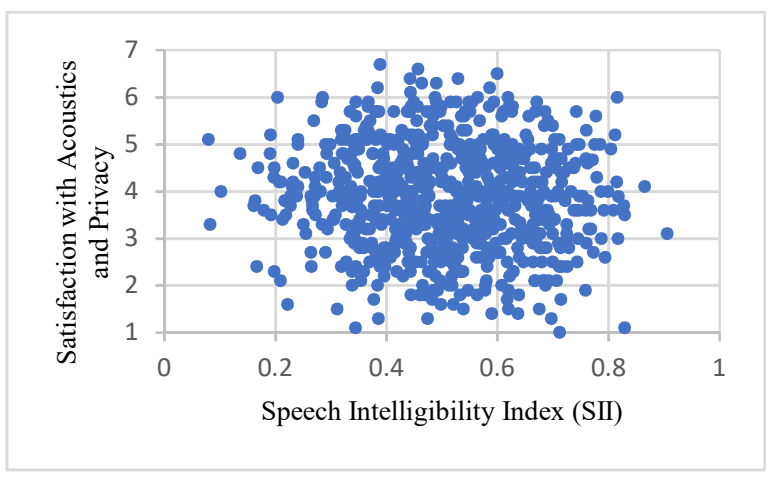

(a) COPE Sample

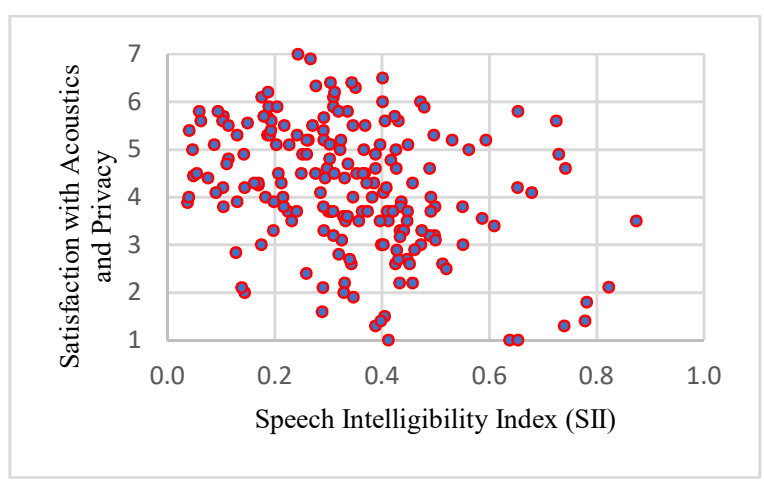

(b) Conventional GPOE + NCR Sample

Figure 10. Satisfaction with acoustics and privacy vs. speech intelligibility

Table 19 summarizes the analysis results for satisfaction with acoustics and privacy using unaltered physical predictors and satisfaction measures. Various demographic factors were shown to have a statistically significant effect on acoustics satisfaction in these analyses. In the COPE sample, age was shown to have an influence on satisfaction with acoustics and privacy, with younger respondents reporting higher levels of satisfaction. This result contradicts the findings of some other researchers (e.g. Bluyssen, Aries, and van Dommelen 2011; Klitzman and Stellman 1989), and was also not observed within the conventional GPOE + NCR sample. In observing the demographic differences between the data samples (refer to Table 9), the COPE 
sample had more younger respondents which could have contributed to the observed result. The GPOE + NCR dataset also showed sex as a significant predictor variable in the first step of the regression, but this effect did not persist through the rest of the analysis with the inclusion of workstation and IEQ factors. This could be due to unobserved intercorrelations between sex and the other predictor variables. Nonetheless, these results suggest that demographic differences in the respondents can have an effect on acoustics satisfaction, albeit a small one. Researchers should be conscious of the demographic differences in the samples of their studies.

Table 19 - Satisfaction with acoustics and privacy regression results (a) COPE Sample $(N=710)$

\begin{tabular}{lcccc}
\hline & $\boldsymbol{\beta}$ & $\boldsymbol{\beta}$ & $\boldsymbol{\beta}$ & Tolerance \\
\hline Age & $-0.09^{*}$ & $-0.11^{* *}$ & $-0.11^{* *}$ & 0.93 \\
Sex & 0.01 & 0.01 & 0.01 & 0.99 \\
Window & & -0.02 & -0.02 & 0.90 \\
MinPH & & $0.14^{* * *}$ & $0.13^{* *}$ & 0.65 \\
AW & & & -0.01 & 0.45 \\
SII & & & -0.05 & 0.48 \\
\hline $\mathrm{R}^{2}$ Change & 0.01 & $0.02^{* * *}$ & 0.00 & \\
Total R & & $0.03^{* * *}$ & $0.03^{* *}$ & \\
Adjusted $\mathrm{R}^{2}$ & 0.01 & $0.02^{* * *}$ & $0.02^{* *}$ & \\
\hline
\end{tabular}

(b) Conventional GPOE + NCR Sample (N=191)

\begin{tabular}{lcccc}
\hline & $\boldsymbol{\beta}$ & $\boldsymbol{\beta}$ & $\boldsymbol{\beta}$ & Tolerance \\
\hline Age & -0.09 & -0.13 & -0.13 & 0.96 \\
Sex & $0.15^{*}$ & 0.13 & 0.11 & 0.97 \\
Window & & 0.04 & 0.06 & 0.97 \\
WSEnclosure & & $0.34^{* * *}$ & $0.20^{*}$ & 0.63 \\
AW & & & -0.10 & 0.77 \\
SII & & & $-0.26^{* *}$ & 0.61 \\
\hline $\mathrm{R}^{2}$ Change & 0.03 & $0.12^{* * *}$ & $0.04^{*}$ & \\
Total R & 0.03 & $0.15^{* * *}$ & $0.19^{* * *}$ & \\
Adjusted $\mathrm{R}^{2}$ & 0.02 & $0.13^{* * *}$ & $0.16^{* * *}$ & \\
\hline
\end{tabular}

Note: Outcome variable is satisfaction with acoustics and privacy. Tolerance values are shown for variables at the final step. ${ }^{*} \mathrm{p} \leq 0.05,{ }^{*} \mathrm{p} \leq 0.01, * * \mathrm{p} \leq 0.001$. 
The most consistent finding from the results of the acoustics regression was the correlation between more enclosed workstations and higher satisfaction with acoustics and privacy. While not a large effect, more enclosed workstations was consistently observed to be statistically significant across every step of the hierarchical regression in both datasets.

Occupants with higher wall partitions in the COPE sample, and occupants in private enclosed offices in the GPOE + NCR sample both reported higher levels of satisfaction with acoustics \& privacy. These results corroborate with the findings of prior work (e.g. Frontczak et al. 2012; Kim and de Dear 2013), and is interesting because industry trends continue to move practice away from private to more open workstations. The desire to have more open workstation is largely driven by cost-savings on real estate and the belief that more open workspaces support more communication amongst coworkers and fosters innovation (Waber, Magnolfi, \& Lindsay, 2014). However, this belief is largely unsupported by research objective research (Bernstein \& Turban, 2018; Kim \& de Dear, 2013).

In terms of the physical acoustics measurements, neither background noise levels nor speech intelligibility were statistically significant predictors in the COPE model. The conventional GPOE + NCR dataset showed measured speech intelligibility index (SII) to have an effect on satisfaction with acoustics and privacy. Respondents in workstations with lower measured speech intelligibility reported higher satisfaction with acoustics. This result makes logical sense, as occupants who can perceive less of the speech in adjacent spaces are less distracted and can be more focussed on their tasks. Background noise was not found to be a significant predictor in either dataset. It is possible that sound levels within a specific range better captures the benefits to acoustics satisfaction, as opposed to sound levels skewed towards either one of the 'too quiet' or 'too loud' ends of the spectrum. This has been prescribed in office 
acoustics standards such as ASA S12.2 (2008), which recommends a background noise level of 44-48 dBA. Other researchers have made similar recommendations as a result of their findings. Bradley and Gover (2004) have suggested a background noise level of $45 \mathrm{dBA}$ for sound masking, with $48 \mathrm{dBA}$ deemed too loud. This will be discussed further in the analysis for research question $\# 2$, where the benefits of a specific range of background noise level (i.e. upper and lower thresholds of noise) is explored.

The differences in the results between the two datasets is intriguing. In observing the descriptive statistics in Table 12 and Figure 10, it can be seen that the COPE sample had generally higher speech intelligibility as well as lower variance in the measurements, which can diminish correlations in the data. Even though the GPOE+NCR sample had fewer cases, a wider and more varied range of SII values were captured. This helps to explain the observed relationships between speech intelligibility and satisfaction, particularly across the lower measured ranges. It is likely that the inclusion of private workstations in the GPOE+NCR resulted in the lower observed SII measurements.

In extending the analysis of acoustic conditions to the non-linear trends in the data, Table 20 summarizes the sub-grouped acoustics regression results. The regression models in the COPE model had limited statistically significant predictors of acoustics satisfaction, and very little variance was explained. The previously observed effects of age and partition height were not seen in these results. This suggests that the influences of these factors are perceived across the entire satisfaction spectrum, but their effects were small. Thus, when analysed separately using smaller sample sizes within specific satisfaction ranges, they were not found to be statistically significant. 
Table 20 - Sub-grouped (asymmetric) acoustics and privacy regression results

\begin{tabular}{|c|c|c|c|c|c|c|c|c|c|}
\hline \multirow[t]{2}{*}{ (a) } & \multicolumn{3}{|c|}{$\begin{array}{c}\text { COPE - Acou_Dissat } \\
(N=168)\end{array}$} & \multicolumn{3}{|c|}{$\begin{array}{c}\text { COPE - Acou_Neutral } \\
(N=40 \overline{\overline{9}})\end{array}$} & \multicolumn{3}{|c|}{ COPE - Acou_Sat $(N=133)$} \\
\hline & $\beta$ & $\beta$ & $\beta$ & $\beta$ & $\beta$ & $\beta$ & $\beta$ & $\beta$ & $\beta$ \\
\hline Age & -0.03 & -0.06 & -0.07 & 0.06 & 0.04 & 0.04 & 0.11 & 0.09 & 0.11 \\
\hline Sex & -0.02 & -0.02 & -0.02 & -0.08 & -0.08 & -0.08 & 0.04 & 0.00 & 0.00 \\
\hline Window & & -0.02 & 0.00 & & 0.02 & 0.03 & & $0.29^{* * *}$ & $0.26^{* *}$ \\
\hline $\mathrm{MinPH}$ & & 0.11 & 0.03 & & 0.07 & 0.05 & & -0.01 & 0.08 \\
\hline AW & & & -0.15 & & & -0.06 & & & 0.22 \\
\hline SII & & & -0.04 & & & -0.03 & & & 0.19 \\
\hline $\mathrm{R}^{2}$ Change & 0.00 & 0.01 & 0.01 & 0.01 & 0.01 & 0.00 & 0.02 & $0.08 * *$ & 0.02 \\
\hline Total $\mathrm{R}^{2}$ & 0.00 & 0.01 & 0.02 & 0.01 & 0.02 & 0.02 & 0.02 & $0.10^{*}$ & $0.12 *$ \\
\hline Adjusted $\mathrm{R}^{2}$ & -0.01 & -0.01 & -0.01 & 0.01 & 0.01 & 0.00 & 0.00 & $0.07^{*}$ & $0.08^{*}$ \\
\hline \multirow[t]{2}{*}{ (b) } & & & & \multicolumn{3}{|c|}{$\begin{array}{c}G P O E+N C R-A c o u \_D i s N e u \\
(N=127)\end{array}$} & \multicolumn{3}{|c|}{$\begin{array}{c}G P O E+N C R-A c o u \_S a t \\
(N=64)\end{array}$} \\
\hline & & & & $\beta$ & $\beta$ & $\beta$ & $\beta$ & $\beta$ & $\beta$ \\
\hline Age & & & & -0.14 & -0.15 & -0.15 & 0.22 & $0.28^{*}$ & $0.29 *$ \\
\hline Sex & & & & $0.19^{*}$ & 0.16 & 0.12 & 0.01 & -0.01 & 0.00 \\
\hline Window & & & & & 0.07 & 0.11 & & -0.22 & -0.22 \\
\hline WSEnclosure & & & & & $0.23 * *$ & -0.01 & & -0.01 & 0.02 \\
\hline AW & & & & & & $-0.24 *$ & & & 0.04 \\
\hline SII & & & & & & $-0.39 * * *$ & & & 0.07 \\
\hline $\mathrm{R}^{2}$ Change & & & & $0.05^{*}$ & $0.06^{*}$ & $0.10^{* * *}$ & 0.05 & 0.04 & 0.00 \\
\hline Total $\mathrm{R}^{2}$ & & & & $0.05^{*}$ & $0.11^{* *}$ & $0.21 * * *$ & 0.05 & 0.09 & 0.10 \\
\hline Adjusted $\mathrm{R}^{2}$ & & & & $0.03 *$ & $0.08 * *$ & $0.17^{* * *}$ & 0.02 & 0.03 & 0.00 \\
\hline
\end{tabular}

Note: Outcome variable is satisfaction with acoustics and privacy. ${ }^{*} \mathrm{p} \leq 0.05,{ }^{* *} \mathrm{p} \leq 0.01,{ }^{* * *} \mathrm{p} \leq$ 0.001 .

Within the satisfied sub-group of the COPE sample, the results showed window proximity have a statistically significant effect. This result was not previously observed in the overall regression model and suggests that window proximity behaved like a bonus factor in this respondent group. It is not immediately clear why window proximity increased the satisfaction of already satisfied occupants even more. This could be an artefact of the sub-grouping of the dataset by occupant satisfaction. Another explanation could be the component environmental satisfaction measures used in the analysis. These composite factors were previously been 
developed and analyzed by others, and the three aspects of environmental satisfaction were shown to be intercorrelated (Veitch, Farley, and Newsham 2002). It could be these intercorrelations between the individual components of environmental satisfaction that resulted in the observed benefits of windows. For instance, it is possible that the benefits of feeling more connected with the outdoor (as a result of window proximity) could have indirectly affected multiple aspects of occupant satisfaction (e.g. lighting and acoustics). Future research is recommended to confirm the repeatability of this observation with other respondent groups.

As previously stated, the dissatisfied and neutral sub-groups were combined in the GPOE + NCR sample due to sample size restrictions. This model partially confirmed the previously observed effects of sex and workstation enclosure in Table 19. However, their effects did not persist with the inclusion of background noise and speech intelligibility in the model, which were both found to have statistically significant effects. This suggests that these acoustic factors behave more as basic factors and have a larger effect on reducing dissatisfaction than improving the satisfaction of already satisfied occupants. Occupants with quieter environments and less intelligible speech from adjacent spaces reported less dissatisfaction with acoustics and privacy. Other researchers have reported similar findings, with studies suggesting lower speech intelligibilities in office spaces and an 'acceptable' SII limit threshold at 0.2 (Bradley 2003).

The results for the satisfied occupants in the GPOE + NCR sample show that age had a statistically significant effect, suggesting that it acts as a bonus factor. When already satisfied, older occupants reported even higher levels of satisfaction than younger occupants. This is in agreement of the findings of others (e.g. Bluyssen, Aries, and van Dommelen 2011; Klitzman and Stellman 1989), but contradicts the previous COPE results shown in Table 19. A possible explanation of this observation could be that older occupants likely have a more senior role 
within their respective organizations. As such they are more likely to be housed in private, enclosed offices. This effect could have been diminished in the COPE dataset as that sample only contained open-plan offices and did not contain private workstations, possibly explaining the observed statistical insignificance.

The regression results with respect to compliance with the ASA S12.2 (2008) acoustics standard, as outlined in research question $\# 2$ are shown below in Table 21 . The results for the first two blocks of this regression table are identical to that of the overall acoustics regression model (Table 19), as the sample and inputs for demographics and workstation factors were identical. These factors were left in the regression model to be consistent with previous sections and maintain control of the confounding variables. The observations for the demographic and workstation characteristics are not re-iterated here. In the third block of the COPE dataset, SoundBelow and SoundAbove were input as predictor variables, representing background noise levels below, and above the recommended 44-48 dBA range, respectively. As previously stated, only SoundBelow was used in the GPOE+NCR dataset to preserve a valid sample size. 
Table 21 - Satisfaction with acoustics and privacy regression results for IEQ guidelines (a) COPE Sample $(N=715)$

\begin{tabular}{lcccc}
\hline & $\boldsymbol{\beta}$ & $\boldsymbol{\beta}$ & $\boldsymbol{\beta}$ & Tolerance \\
\hline Age & $-0.09^{*}$ & $-0.11^{* *}$ & $-0.11^{* *}$ & 0.93 \\
Sex & 0.01 & 0.01 & 0.01 & 1.00 \\
Window & & -0.02 & -0.02 & 0.92 \\
MinPH & & $0.14^{* * *}$ & $0.14^{* * *}$ & 0.88 \\
SoundBelow & & & 0.01 & 0.78 \\
SoundAbove & & & 0.01 & 0.76 \\
\hline $\mathrm{R}^{2}$ Change & 0.01 & $0.02^{* * *}$ & 0.00 & \\
Total $\mathrm{R}^{2}$ & 0.01 & $0.03^{* * *}$ & $0.03^{* *}$ & \\
Adjusted $\mathrm{R}^{2}$ & 0.01 & $0.02^{* * *}$ & $0.02^{* *}$ & \\
\hline
\end{tabular}

(b) Conventional GPOE + NCR Sample (N=191)

\begin{tabular}{lcccc}
\hline & $\boldsymbol{\beta}$ & $\boldsymbol{\beta}$ & $\boldsymbol{\beta}$ & Tolerance \\
\hline Age & -0.09 & -0.13 & -0.13 & 0.96 \\
Sex & $0.15^{*}$ & 0.13 & 0.13 & 0.98 \\
Window & & 0.04 & 0.04 & 0.97 \\
WSEnclosure & & $0.34^{* * *}$ & $0.35^{* * *}$ & 0.97 \\
SoundBelow & & & -0.05 & 0.99 \\
\hline $\mathrm{R}^{2}$ Change & 0.03 & $0.12^{* * *}$ & 0.00 & \\
Total $\mathrm{R}^{2}$ & 0.03 & $0.15^{* * *}$ & $0.15^{* * *}$ & \\
Adjusted $\mathrm{R}^{2}$ & 0.02 & $0.13^{* * *}$ & $0.13^{* * *}$ & \\
\hline
\end{tabular}

Note: Outcome variable is satisfaction with acoustics and privacy. Tolerance values are shown for variables at the final step. ${ }^{*} \mathrm{p} \leq 0.05,{ }^{* *} \mathrm{p} \leq 0.01,{ }^{* * *} \mathrm{p} \leq 0.001$.

Contrary to expectations, no significant differences in satisfaction between measured sound levels within, and outside of, ASA S12.2 (2008) recommended thresholds were observed. In a study using slightly different noise level intervals and analysis methods, Newsham et al. (2008) also found background noise to not significantly influence acoustic satisfaction. One possible explanation for this observation is that the range of measured sound levels within our data was limited. Only a small number of workstations had measured sound levels in the extreme ends of the spectrum. In particular, the GPOE+NCR sample had very low measured sound levels, with a mean of $42 \mathrm{dBA}$ (refer to Table 12). This result could also be because other 
acoustics factors such as speech intelligibility, which were not included in this regression model, were more influential in terms of predicting satisfaction with acoustics and privacy.

The acoustics results generally had better-fitting models than the previous lighting satisfaction models in terms of effect size $\left(\mathrm{R}^{2}\right)$. It was observed in the various acoustics regressions above (Tables 19-21) that the effect sizes of the overall models were generally small to medium, with the largest explained variance noted at $21 \%$. While still not very predictive, other POE studies have reported similar effect sizes in their analysis of workstation acoustics. For instance, Leder et al., (2016) reported $\mathrm{R}^{2}$ values of 0.05 to 0.23 in their regression models. Using a different regression procedure, Newsham et al. (2008) reported pseudo- $\mathrm{R}^{2}$ (approximated estimate of $\mathrm{R}^{2}$ ) values of 0.20 . Still, while the results do not show that the input variables are the sole contributors to acoustics satisfaction, various statistically significant predictor variables were observed in the models, suggesting that they have some beneficial effects.

Where statistically significant, the demographic factors generally had a smaller effect size than that of the workstation characteristics and physical acoustics measurements. The workstation characteristics in the COPE sample also had a smaller explained variance than the GPOE + NCR sample, suggesting that increases in workstation height explained less in the improvements to satisfaction than having an enclosed office with full height walls and a door.

The results generally showed the workstation characteristics (i.e. workstation enclosure) to have a larger effect size than the physical acoustics measurements, suggesting that occupants are more perceptive of the benefits of more enclosed workstations than the actual acoustics levels in the workspace. However, this was not observed in the sub-grouped dissatisfied GPOE+NCR model. Measured sound level and speech intelligibility explaining $10 \%$ of the total variance 
(medium effect size), compared to workstation characteristics, which explained only $6 \%$. This result suggests that in an office with a mixed open and private workstation layout, improvements to sound levels and speech intelligibility have a larger influence on reducing acoustics dissatisfaction in the occupants than workstation configurations.

Although the observed effect sizes and statistical significance were varied amongst the various regression models, the results generally showed that occupants preferred more quiet environments with less speech noises. Where possible, designers should consider the use of more sound absorbent interior finish materials, as well as better acoustic optimization within the office spaces. The results further highlight the beneficial effects of more enclosed workstations; higher partitions and more private workstations should be considered for their benefits on occupant satisfaction with acoustics and privacy.

\subsection{Thermal Conditions}

The analysis results regarding the thermal conditions in the occupants' workstation are presented in this section. Like the results of the other aspects of IEQ, visualizations of the data were generated prior to performing any statistical analyses of the data. Graphs of satisfaction with temperature \& ventilation versus measured thermal conditions were generated to observe any obvious trends or patterns in the data. Figures 11 to 14 below show the graphs for both datasets. Similar to the other graphs, the plotted data was very scattered and no obvious trends or patterns emerged. 


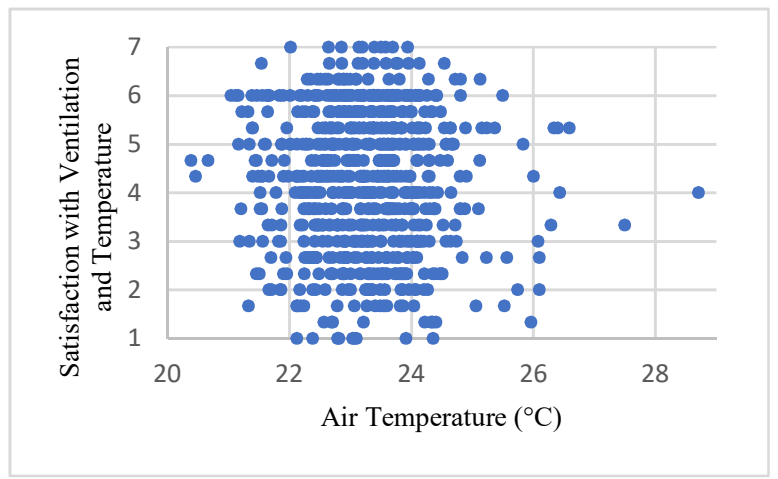

(a) COPE Sample

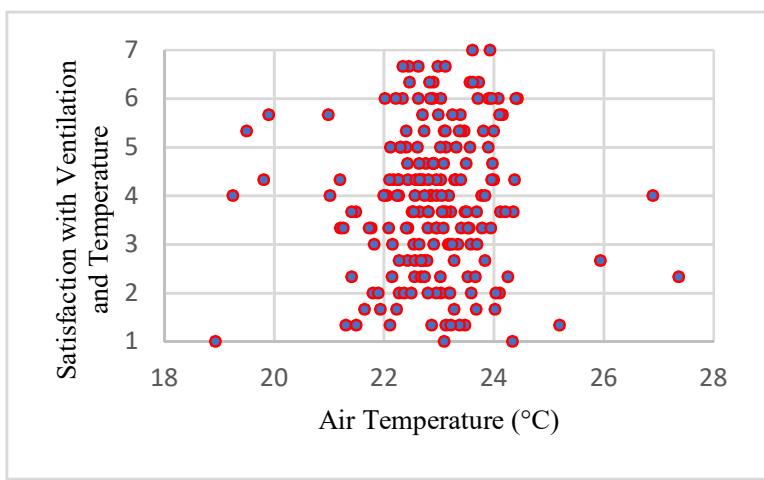

(b) Conventional GPOE + NCR Sample

Figure 11. Satisfaction with ventilation and temperature vs. air temperature

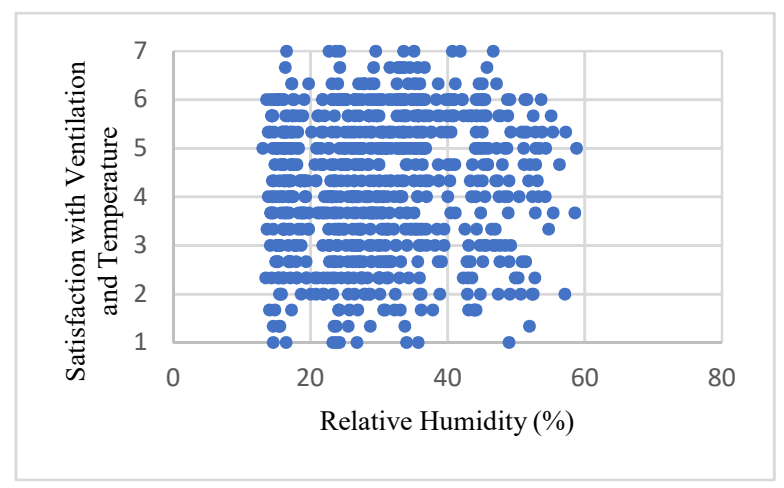

(a) COPE Sample

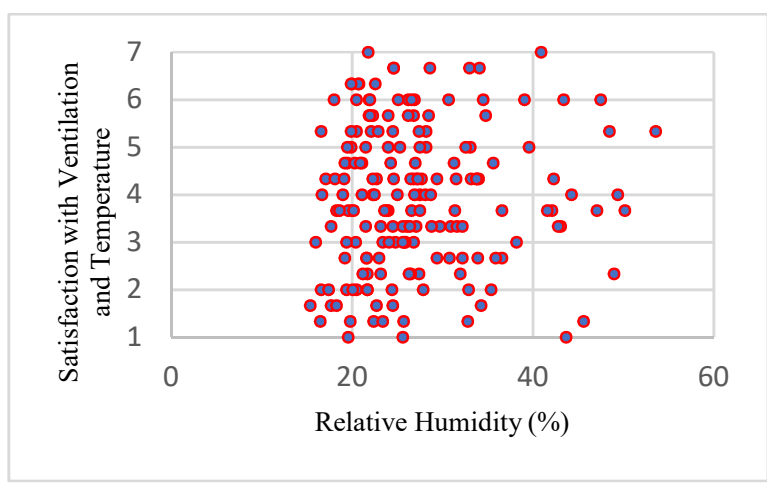

(b) Conventional GPOE + NCR Sample

Figure 12. Satisfaction with ventilation and temperature vs. relative humidity

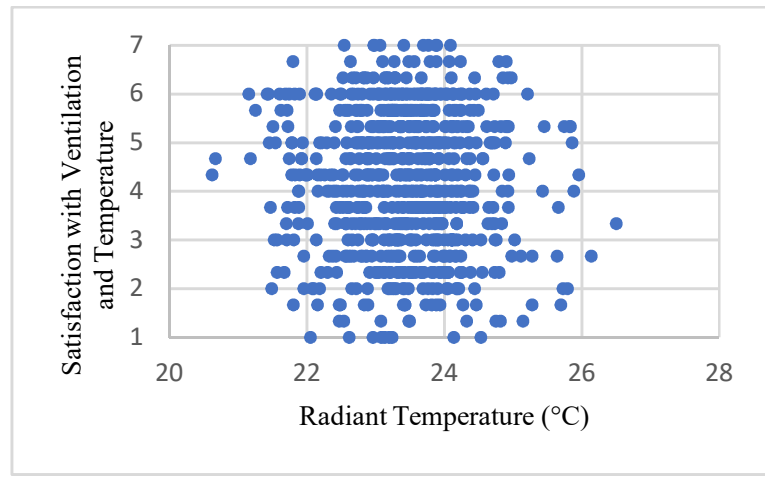

(a) COPE Sample

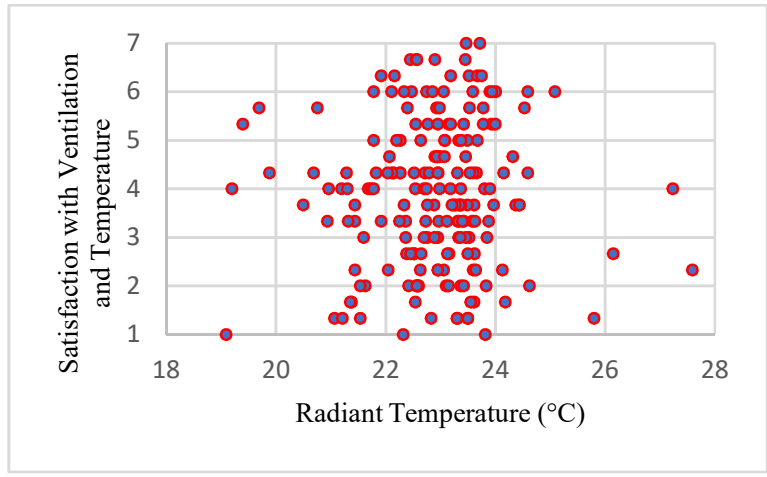

(b) Conventional GPOE + NCR Sample

Figure 13. Satisfaction with ventilation and temperature vs. radiant temperature 


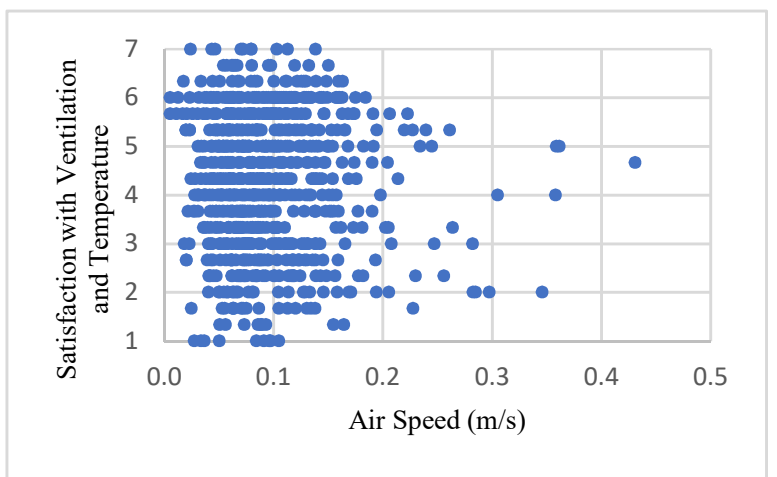

(a) COPE Sample

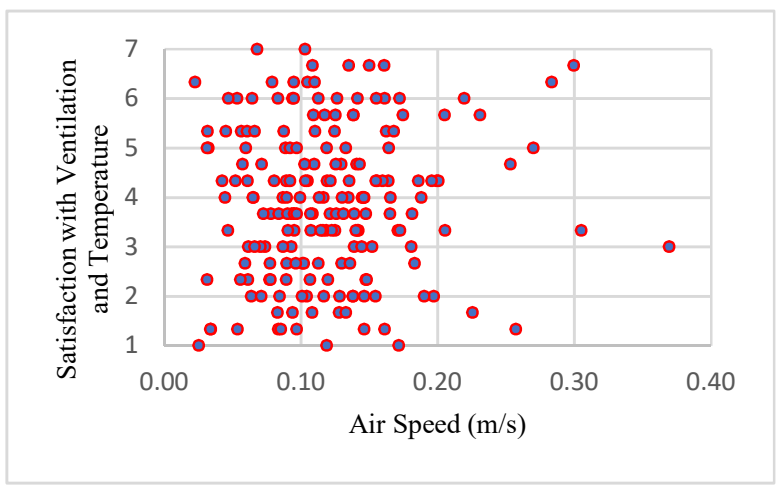

(b) Conventional GPOE + NCR Sample

Figure 14. Satisfaction with ventilation and temperature vs. air velocity

Table 22 summarizes the results for satisfaction with ventilation and temperature using the unaltered physical predictors and satisfaction measures. Demographic factors had a small, but statistically significant effect on thermal satisfaction in both data samples. The regression results showed that male occupants reported higher satisfaction with ventilation and temperature than their female counterparts in both datasets. This is largely consistent with literature, and other researchers have reported similar findings in their studies (Bluyssen et al., 2011; Karjalainen, 2007; Kim et al., 2013). This observed result may be related to several reasons such as biological differences (i.e. metabolic rates) between the sexes, or differences in standard clothing attires (i.e. insulation), particularly during the cooling season when the $\mathrm{A} / \mathrm{C}$ is in operation (Choi et al., 2010; Kim et al., 2013). 
Table 22 - Satisfaction with ventilation and temperature regression results (a) COPE Sample $(N=756)$

\begin{tabular}{lcccc}
\hline & $\boldsymbol{\beta}$ & $\boldsymbol{\beta}$ & $\boldsymbol{\beta}$ & Tolerance \\
\hline Age & -0.05 & -0.01 & -0.03 & 0.92 \\
Sex & $0.21^{* * *}$ & $0.21^{* * *}$ & $0.24^{* * *}$ & 0.96 \\
Window & & $-0.09^{*}$ & $-0.11^{* *}$ & 0.92 \\
MinPH & & $-0.16^{* * *}$ & $-0.18^{* * *}$ & 0.81 \\
AirTemp & & $-0.12^{* * *}$ & 0.87 \\
RH & & & 0.07 & 0.86 \\
AirVelocity & & & $-0.09^{*}$ & 0.90 \\
\hline $\mathrm{R}^{2}$ Change & $0.05^{* * *}$ & $0.04^{* * *}$ & $0.03^{* * *}$ & \\
Total $\mathrm{R}^{2}$ & $0.05^{* * *}$ & $0.08^{* * *}$ & $0.11^{* * *}$ & \\
Adjusted $\mathrm{R}^{2}$ & $0.04^{* * *}$ & $0.08^{* * *}$ & $0.10^{* * *}$ & \\
\hline
\end{tabular}

(b) Conventional GPOE + NCR Sample (N=191)

\begin{tabular}{lcccc}
\hline & $\boldsymbol{\beta}$ & $\boldsymbol{\beta}$ & $\boldsymbol{\beta}$ & Tolerance \\
\hline Age & -0.04 & -0.02 & -0.02 & 0.94 \\
Sex & $0.19^{* *}$ & $0.20^{* *}$ & $0.21^{* *}$ & 0.98 \\
Window & & -0.01 & -0.02 & 0.96 \\
WSEnclosure & & $-0.17^{*}$ & $-0.18^{*}$ & 0.95 \\
AirTemp & & 0.01 & 0.97 \\
RH & & & 0.05 & 0.96 \\
AirVelocity & & & 0.06 & 0.97 \\
\hline $\mathrm{R}^{2}$ Change & $0.04^{*}$ & $0.03^{*}$ & 0.01 & \\
Total R & $0.04^{2}$ & $0.07^{*}$ & $0.08^{*}$ & \\
Adjusted R & $0.03^{*}$ & $0.05^{*}$ & $0.04^{*}$ & \\
\hline
\end{tabular}

Note: Outcome variable is satisfaction with ventilation and temperature. Tolerance values are shown for variables at the final step. ${ }^{*} p \leq 0.05,{ }^{* *} p \leq 0.01, * * * p \leq 0.001$.

Workstation factors were also found to influence satisfaction with ventilation and temperature. Workstation enclosure, represented by partition height in the COPE model and private enclosed workstations in the GPOE+NCR model, were statistically significant effects in the analysis. In both models, occupants in more enclosed workstations reported lower levels of satisfaction. Most researchers have generally observed similar results in their studies (Charles et al., 2006; Kim \& de Dear, 2013). A possible explanation for this observation is that taller (and 
full height) walls could prevent good air mixing and thermal conditioning throughout the spaces from the mechanical systems. Window proximity was also found to be a significant predictor in the COPE sample, with occupants of window offices reporting lower satisfaction with ventilation and temperature. This could due to a combination of several possible issues, such as radiant asymmetry, air leakage and drafts in the windows, and poor thermal performance of the window units. The effects of window proximity were not observed in the GPOE+NCR sample, possibly due to the smaller sample size, as well as improvements in window performance over the years (higher R-value, less leaky etc.).

Air temperature and air velocity both had an influence on satisfaction in the COPE sample, where lower recorded levels in both parameters correlated to higher satisfaction with ventilation and temperature. These effects were not observed in the GPOE+NCR sample, and no measured thermal IEQ variables were statistically significant predictors. The COPE sample had, on average, marginally warmer thermal environments (Table 12) which could have contributed to the observed result in which occupants preferred cooler environments. The COPE study also took place nearly two decades ago, and it is possible that the typical dress codes within organizations were different and less flexible than they are today. It is plausible that the clothing attire expectations during this period were more traditional (e.g. suit jacket versus dress shirts or polos for males) and provided more insulation than typical office attires nowadays, resulting in a preference of cooler environments. It is also easier for occupants to adjust their clothing levels by adding additional layers in cooler environments. When the temperature is too warm, there is are limitations to how much clothing can be acceptably removed in an office environment. Other studies have also found benefits to cooler temperatures and thermal perceptions on occupants (Yeom \& Delogu, 2019). 
The COPE sample also had a higher proportion of male respondents than the GPOE+NCR sample. Previous studies have generally found that females prefer warmer thermal environments compared to men (Choi et al., 2010), possibly due to the previously aforementioned metabolic and clothing differences. As the GPOE+NCR sample had a larger ratio of female respondents than male, it is possible that the warmer thermal environment preferences of women led to the lack of significance in the measured thermal IEQ predictors.

The relationship between thermal conditions and occupant satisfaction in standard office environments is one that is not clear-cut. Some studies suggest that while the varying thermal conditions affect thermal perceptions (e.g. respondent reports feeling warm), they did not find subsequent impacts on satisfaction (Lakeridou et al., 2012). This phenomenon could have further contributed to the small variance explained by the measured thermal parameters on occupant satisfaction.

In extending the analysis of the thermal environment to the non-linear trends in the data, Table 23 summarizes the sub-grouped ventilation and temperature regression results. There were generally fewer statistically significant relationships in both the COPE and GPOE+NCR model compared to Table 22. Still, there were some notable and interesting observations within the results. 
Table 23 - Sub-grouped (asymmetric) ventilation and temperature regression results

\begin{tabular}{|c|c|c|c|c|c|c|c|c|c|}
\hline \multirow[t]{2}{*}{ (a) } & \multicolumn{3}{|c|}{$\begin{array}{c}\text { COPE }- \text { Temp_Dissat } \\
(N=18 \overline{6})\end{array}$} & \multicolumn{3}{|c|}{$\begin{array}{c}\text { COPE - Temp Neutral } \\
(N=26 \overline{2})\end{array}$} & \multicolumn{3}{|c|}{ COPE - Temp_Sat $(N=308)$} \\
\hline & $\boldsymbol{\beta}$ & $\beta$ & $\beta$ & $\beta$ & $\beta$ & $\beta$ & $\beta$ & $\beta$ & $\beta$ \\
\hline Age & -0.01 & 0.02 & 0.01 & -0.07 & $\begin{array}{l}-0.09 \\
\end{array}$ & $\begin{array}{l}-0.09 \\
\end{array}$ & $0.12 *$ & $0.15^{* *}$ & $0.14 *$ \\
\hline Sex & 0.08 & 0.09 & 0.10 & $0.12^{*}$ & $0.12^{*}$ & $0.13^{*}$ & 0.09 & 0.09 & $0.12^{*}$ \\
\hline Window & & -0.07 & -0.08 & & 0.11 & 0.09 & & $-0.15^{* *}$ & $-0.16^{* *}$ \\
\hline MinPH & & -0.09 & -0.10 & & -0.01 & -0.03 & & -0.06 & -0.10 \\
\hline AirTemp & & & -0.03 & & & -0.10 & & & -0.11 \\
\hline RH & & & 0.00 & & & 0.05 & & & -0.01 \\
\hline AirVelocity & & & -0.03 & & & 0.04 & & & -0.11 \\
\hline $\mathrm{R}^{2}$ Change & 0.01 & 0.02 & 0.00 & 0.01 & 0.01 & 0.01 & $0.02 *$ & $0.03^{*}$ & 0.02 \\
\hline Total $\mathrm{R}^{2}$ & 0.01 & 0.02 & 0.03 & 0.02 & 0.03 & 0.04 & $0.02 *$ & $0.05 * *$ & $0.07 * *$ \\
\hline Adjusted $\mathrm{R}^{2}$ & -0.01 & 0.00 & -0.01 & 0.01 & 0.02 & 0.02 & $0.02 *$ & $0.04 * *$ & $0.05^{* *}$ \\
\hline \multirow[t]{2}{*}{ (b) } & \multicolumn{3}{|c|}{$\begin{array}{c}G P O E+N C R- \\
\text { Temp_Dissat }(N=60)\end{array}$} & \multicolumn{3}{|c|}{$\begin{array}{c}G P O E+N C R- \\
\text { Temp_Neutral }(N=77)\end{array}$} & \multicolumn{3}{|c|}{$\begin{array}{c}G P O E+N C R-T e m p \_S a t \\
(N=54)\end{array}$} \\
\hline & $\beta$ & $\beta$ & $\beta$ & $\beta$ & $\beta$ & $\beta$ & $\beta$ & $\beta$ & $\beta$ \\
\hline Age & -0.06 & -0.04 & -0.01 & 0.04 & 0.05 & 0.04 & 0.12 & 0.13 & 0.12 \\
\hline Sex & $0.26^{*}$ & $0.27^{*}$ & $0.26^{*}$ & 0.08 & 0.07 & 0.05 & 0.13 & 0.13 & 0.16 \\
\hline Window & & -0.10 & -0.13 & & -0.11 & -0.09 & & -0.04 & -0.02 \\
\hline WSEnclosure & & -0.18 & -0.18 & & 0.08 & 0.09 & & -0.07 & -0.04 \\
\hline AirTemp & & & 0.15 & & & 0.00 & & & 0.12 \\
\hline RH & & & -0.01 & & & -0.16 & & & 0.07 \\
\hline AirVelocity & & & 0.11 & & & -0.09 & & & 0.16 \\
\hline $\mathrm{R}^{2}$ Change & 0.07 & 0.05 & 0.03 & 0.01 & 0.02 & 0.04 & 0.04 & 0.01 & 0.04 \\
\hline Total $\mathrm{R}^{2}$ & 0.07 & 0.12 & 0.15 & 0.01 & 0.02 & 0.06 & 0.04 & 0.04 & 0.09 \\
\hline Adjusted $\mathrm{R}^{2}$ & 0.04 & 0.06 & 0.04 & -0.02 & -0.03 & -0.04 & 0.00 & -0.03 & -0.05 \\
\hline
\end{tabular}

Note: Outcome variable is satisfaction with ventilation and temperature. ${ }^{*} \mathrm{p} \leq 0.05,{ }^{* *} \mathrm{p} \leq 0.01$, $* * * \mathrm{p} \leq 0.001$.

In terms of demographic differences, sex had an effect in the dissatisfied GPOE+NCR and the neutral COPE samples, suggesting that it behaves more as a basic factor. The results show that sex has a more significant effect on occupant dissatisfaction, as opposed to satisfaction. Age was also found to be have a statistically significant effect in the satisfied COPE model. This result suggests that within the already satisfied occupants of the COPE sample, older occupants reported higher satisfaction. This effect, while not observed in the other analyses or 
the GPOE+NCR dataset, is in agreement with the results of Choi, Aziz, and Loftness (2010), who found higher thermal satisfaction in occupants over 40 years of age.

The results of the satisfied sub-group in the COPE sample showed window proximity to have a negative effect on satisfaction with ventilation and temperature. Similar effects of window proximity were observed in Table 22, suggesting that window proximity influences occupants across the entire satisfaction spectrum. However, this result appears to be more apparent in already satisfied occupants.

Many of the previously statistically significant IEQ variables in the overall linear regression models (Table 22) were no longer statistically significant in the sub-grouped analyses. In particular, the GPOE+NCR had little statistically significant predictor variables and the models generally explained very little variance in the data. Similar results were generally observed in the analysis of the sub-grouped models of the other aspects of IEQ (lighting, acoustics). As previously mentioned in the discussions of the other IEQ components, these observations could be an artefact of the diminished sample sizes due to sub-grouping. As the sample sizes were generally smaller, this could have reduced the statistical power of the models, leading to a lack of potentially otherwise statistically significant predictors. This could also be the result of the IEQ predictors having a less pronounced but significant effects across the entire satisfaction spectrum. Thus, when the sample was sub-grouped into smaller samples with reduced satisfaction ranges, correlations in the data were diminished.

The regression results with respect to compliance with the ASHRAE Standard 55 (2017) thermal comfort criteria, as outlined in research question \#2 are shown below in Table 24 . The results shown in the first two blocks are identical to that of the overall satisfaction with ventilation and temperature regression model (Table 22), as the sample and inputs for 
demographic and workstation factors were identical. These factors were left in the regression model to be consistent with previous sections and maintain control of the confounding variables. The observations for the demographic and workstation characteristics are not re-iterated here. In the third block of the model, ThermBelow was input as a predictor, representing whether measured thermal environment conditions were below (cooler than) ASHRAE recommendations.

Table 24 - Satisfaction with ventilation and temperature regression results for IEQ guidelines (a) COPE Sample $(N=756)$

\begin{tabular}{lcccc}
\hline & $\boldsymbol{\beta}$ & $\boldsymbol{\beta}$ & $\boldsymbol{\beta}$ & Tolerance \\
\hline Age & -0.05 & -0.01 & -0.02 & 0.94 \\
Sex & $0.21 * * *$ & $0.21 * * *$ & $0.22 * * *$ & 1.00 \\
Window & & $-0.09 *$ & $-0.10^{* *}$ & 0.93 \\
MinPH & & $-0.16^{* * *}$ & $-0.17 * * *$ & 0.93 \\
ThermBelow & & & $0.10^{* *}$ & 0.96 \\
\hline $\mathrm{R}^{2}$ Change & $0.05 * * *$ & $0.04 * * *$ & $0.01 * *$ & \\
Total R & & $0.08^{2} * *$ & $0.09 * * *$ & \\
Adjusted $\mathrm{R}^{2}$ & $0.05^{* * *}$ & $0.08^{* * *}$ & $0.09 * * *$ & \\
\hline
\end{tabular}

(b) Conventional GPOE + NCR Sample $(N=191)$

\begin{tabular}{lcccc}
\hline & $\boldsymbol{\beta}$ & $\boldsymbol{\beta}$ & $\boldsymbol{\beta}$ & Tolerance \\
\hline Age & -0.04 & -0.02 & -0.03 & 0.95 \\
Sex & $0.19^{* *}$ & $0.20^{* *}$ & $0.22^{* *}$ & 0.97 \\
Window & & -0.01 & -0.01 & 0.97 \\
WSEnclosure & & $-0.17^{*}$ & $-0.17^{*}$ & 0.97 \\
ThermBelow & & & 0.09 & 0.96 \\
\hline $\mathrm{R}^{2}$ Change & $0.04^{*}$ & $0.03^{*}$ & 0.01 & \\
Total R & & $0.07^{* *}$ & $0.08^{*}$ & \\
Adjusted R & $0.04^{*}$ & $0.05^{* *}$ & $0.05^{*}$ & \\
\hline
\end{tabular}

Note: Outcome variable is satisfaction with ventilation and temperature. Tolerance values are shown for variables at the final step. ${ }^{*} \mathrm{p} \leq 0.05, * * \mathrm{p} \leq 0.01,{ }^{* * *} \mathrm{p} \leq 0.001$.

Within the GPOE+NCR dataset, there were no differences in satisfaction between measured indoor environments cooler than, and not cooler than, ASHRAE Standard 55. In the COPE dataset, the results show that occupants in environments cooler than the recommended 
range had higher satisfaction with ventilation and temperature. While unexpected, these results are in-line with the findings of others (e.g. Cheung et al. 2019), who found PMV-PPD to be a poor indicator of thermal sensation and satisfaction. These results may be further explained by the fact that there is a limited range of measured thermal conditions, thus diminishing possible correlations in the data. Only a few cases across both datasets had conditions warmer than the prescribed ASHRAE criteria. Also, unlike ASHRAE Standard 55 which focuses purely on thermal comfort, the composite measure of satisfaction with ventilation and temperature used in this analysis refers not only to the thermal environment, but also air quality. While ventilation systems in large North American office buildings typically regulate both air quality and the thermal environment, it is possible that impressions of air quality separate from thermal issues may have diluted any observed effects. Assumptions of clothing and activity levels also may not have been entirely reflective of actual conditions, potentially resulting in inaccurate PMV and PPD calculations. As previously mentioned, there is better adjustability in terms of wearing additional layers of clothing compared to removing clothing, a logical interpretation to why occupants may have a preference to cooler environments.

Like the results of the other aspects of IEQ (lighting, acoustics), small to medium effect sizes in the various satisfaction with ventilation and temperature regression models (Tables 2224) were observed. The largest explained variance $\left(\mathrm{R}^{2}\right)$ noted in the models was 0.11 (or $11 \%$ ). Still, while the results do not show that the input variables are the sole contributors to satisfaction with ventilation and temperature, various statistically significant predictor variables were observed in the models, suggesting that they have some beneficial effects. Other POE studies have often reported similar effect sizes in their analyses. Leder et al., (2016) reported $\mathrm{R}^{2}$ values of 0.14 and 0.18 , while Charles et al., (2006) also reported similar $\mathrm{R}^{2}$ values of 0.14 . These 
analyses generally included many more predictor variables in the models, such as pollutant concentrations. This can explain the marginally improved effect sizes observed in these studies compared to those in this thesis.

The demographic factors (age and sex) typically had larger explained variance (values typically around $4-5 \%$ ) than the workstation and physical thermal measurement parameters. This observation highlights the importance of demographic considerations, particularly differences between the sexes, when performing evaluations of the thermal environment. The differing thermal preferences between the sexes are not only reinforced in the results of this thesis, but have also been documented in various research publications by others (Choi et al., 2010; Kim et al., 2013).

The workstation characteristics (windows and workstation enclosure) generally had larger effect sizes than that of the physical thermal environment measurements. This result suggests that within typical, mechanically ventilated office space conditions, the actual provided thermal environment does not play as large a role as expected. This result can be further explained by the fact that these buildings were generally well-performing and consistent in terms of their measured thermal conditions. For example, in terms of the ASHRAE 55 (2017) criteria the range of measured conditions outside of the limits were relatively small. It is likely that a poorly conditioned building with measured conditions way outside the comfort zone (i.e. more extreme) could have led to larger observed effect sizes in the regression models.

\subsection{Discussion - Physical Workstation Conditions}

Several statistically significant predictor variables were observed in our analysis results of the local workstation conditions. Most of the regression models showed small to medium effect sizes. This is very often the case in studies involving human subjects, particularly those in 
an uncontrolled field environment. This is consistent for these types of studies, and prior studies using similar data collection methodologies have often found explained variances in similar ranges (e.g. Veitch et al. 2005; Charles et al. 2006). However, it does show that there are other factors beyond what was included in these analyses that can influence environmental satisfaction. Still, the statistical significance of some of these results suggest that they have some incremental benefits to occupants.

In general, our results show workstation characteristics such as window proximity and workstation enclosure to have larger effect sizes than that of the physical IEQ variables. This result signifies that within the typical range of conditioned office environments, the physical measured IEQ parameters do not play an enormous role in terms of occupant satisfaction. Occupant satisfaction is more heavily influenced by the workstation layout and positioning of their workstation, than that of the measured indoor environment conditions. This could be due to the fact that the office buildings that was a part of these field studies were well-performing, and had occupants that were generally satisfied with their conditions (mean satisfaction mostly above neutral point of 4). The small range of measured indoor conditions across the buildings meant that there were minimal extreme conditions, reducing potential correlations in the data. It is also possible that the sample size of the study wasn't large enough to establish consistent results in the data.

Furthermore, the influence of workstation enclosure and window proximity on occupant satisfaction is an interesting point of discussion. In the analysis of thermal satisfaction, it was observed that more enclosure and closer proximity to windows had negative effects on occupants. The reverse was observed for lighting and acoustics, where window proximity positive influenced lighting satisfaction, and increased workstation enclosure resulted in higher 
acoustics satisfaction. This result highlights the trade-offs between various workspace and satisfaction parameters; beneficial elements of one aspect of environmental satisfaction may negatively affect others. A properly designed and optimized workspace is essential in ensuring occupants are happy, and that their specific needs are met.

In the analysis of possible non-linear effects of workstation factors on occupant satisfaction, the results generally showed few statistically significant predictor variables. However, results of the regression models did find various workstation variables to behave like basic or bonus factors. Still, inconsistencies in these results were observed between the two datasets used. It has been suggested by Matzler, Fuchs, and Schubert (2004) that relationships between physical workspace factors and occupant satisfaction are dynamic, and can differ across groups and change over time. In essence, what may be a basic factor for one group of occupants may in fact be a bonus factor for another. The cause of this can vary, but Kim and de Dear (2012) have highlighted several possible reasons. An occupant located in a private spacious could have higher expectations for IEQ than other occupants, simply due to what they are accustomed to. They have also suggested that the expectations of the same occupant can change over time. For instance, access to outside views (i.e. window proximity) in which the COPE results suggest behaves more like a bonus factor, can change into a proportional or basic factor as occupants become used to it and take the window for granted. This explains some of the differences, as well as the consistencies, in the observed non-linear analyses results. Overall, the results show that physical workspace elements can have non-linear effects across various groups of respondents. Organizations wishing to improve satisfaction as well as researchers should be cognisant of this fact and understand that different occupants may respond to certain workspace elements in different ways. 
In terms of the published IEQ standards, the results show that staying within the recommended IESNA RP-1-12 criteria of 300-500 lux resulted in higher lighting satisfaction. This difference was only observed in comparison to measured illuminance below 300 lux, suggesting that satisfying the minimum lighting threshold was more important than staying within the maximum. There were no significant benefits in satisfaction within the reference criteria for acoustics and thermal comfort. Surprisingly, indoor conditions cooler than the suggested comfort zone in ASHRAE Standard 55 had significantly higher occupant satisfaction. This could be the result of inadequacies in the standard, however there are certain limitations that prohibit making this definitive statement. As previously mentioned, the sample buildings were generally well-performing, and the range of values for the measured acoustics and thermal environment parameters were generally not extreme. It is entirely possible that in more poorly performing buildings with more extreme measured conditions, the benefits of the ASA S12.2 (2008) and the ASHRAE 55 (2017) standards would be observed.

As the regression models generally showed small effect sizes, a discussion on the possible reasons why more accurate-fitting models were not observed is necessary. Identification of the limitations in the study could help explain the small observed effect sizes as well as some of the unexpected results. Firstly, as part of the data collection procedure outlined in the methodology section of this thesis, physical measurements were performed over a 10-15 minute interval. This method is used consistently in research as a necessary trade-off in order to capture reasonable sample sizes. However, this procedure does assume that the short sampling time is representative of the indoor environment over longer periods. The physical indoor conditions in some workstations can vary significantly both on a daily cycle (for example east or west facing) as well as over different seasons. Contrast with the questionnaires, which were framed with (and 
likely occupants responded with) respect to long-term overall conditions. Therefore, if the 10-15 minute sample was not representative of the overall longer-term conditions of the workstation, this would introduce a source of error into the analyses. Furthermore, as mentioned in Section 4.4, there were some differences in the data collection between the various building evaluation studies. The differences in the time gap between the collection of the questionnaire responses and the physical measurements likely also contributed to the uncertainty in the results. While lighting and background noise levels can reasonably be expected to stay consistent year-long, office thermal conditions can have more fluctuations over time. These fluctuations combined with the time gap in the data collection could have contributed to the differences in the observed results.

People also adapt to make themselves more comfortable. There could potentially be such adaptations that were not measured as part of this methodology. In terms of acoustics, occupants could wear headphones or change their position within a workstation to avoid loud spots. Similar considerations could apply to the thermal environment. While the assumptions of clothing value and metabolic rates used to calculate PMV and PPD in the analysis of ASHRAE 55 are typical for indoor office work conditions, they might not be entirely representative of reality. Occupants can modify their thermal sensations by wearing additional or fewer layers of clothing to make themselves more comfortable. In an office environment, there is almost always a dress code, be it implicit or explicit. As such there is a lower threshold in terms of what the occupants can wear at the office and how many layers can be removed, which can explain the observation where occupants reported improved satisfaction at lower measured temperatures. Lighting levels can also be easily adjusted by the occupants with the use of individual task lights or shading devices, or occupants might find specific locations within a workstation to avoid glare. While the 
presence of these features were manually recorded as part of the data collection, adjustments made by occupants using these elements were not captured. For instance, if a task light was off during the time of the data collection, they were left in that configuration for the measurement and occupant use of the task light was not measured.

Given the improved availability of relatively cheap sensors in recent years, perhaps adjustments to the data collection methodology could be made to provide more insights in future POE studies. For example, the use of longitudinal sensors that take measurements over extended periods could alleviate some of the issues regarding fluctuations in the indoor environment conditions over time. Wearable sensors are another option and can provide more accurate data relevant to individual occupant behaviours. For instance, they can help provide more realistic estimates of metabolic activity based on heart rate, and provide more detailed information on individual behaviours and habits.

Another possible reason for the explained results is that humans are very complex in nature. It is possible that other factors not directly related to the workstation conditions could have influenced occupant perceptions of the workplace environment. For example, if an occupant was not satisfied with their job or was having other unrelated issues, they could have poor perceptions of their workstation environment even though the two are seemingly unrelated. So while multiple hierarchical regression is an established statistical technique that has been used in similar studies of the indoor environment (e.g. Leder et al. 2016; Charles et al. 2006), it only analyzes direct relationships between the predictor and outcome parameters. It could be that there are these indirect relationships between certain parameters that are not captured in regression models. Other techniques such as structural equation modelling or other machine 
learning techniques may be able to show additional inter-relationships. This further highlights the complexities of this field, and the need for future research into the area. 


\section{Broader Amenities Outside the Workstation - Results}

In the next section of the thesis, the scope of the analysis is extended to factors beyond the immediate workstation (i.e. building amenities). The results of the broader amenities are presented in the following sections, organized according to the outcome measure. Appendix B shows the associated bivariate correlation tables of the predictor variables. Following the approach used in the previous sections, demographics (age and sex) were entered on the first step, workstation characteristics (window, enclosure, and assignment) on the second step, satisfaction with aspects of indoor environment entered on the third step, and satisfaction with amenities on the fourth and final step. This focus of this thesis section is not the detailed discussion of each individual step of the analysis for each outcome variable, but rather to expand upon previous studies where only immediate workstation parameters were considered, and to highlight trends across all analyses.

\subsection{Data Reduction}

In extending the analyses to amenities factors outside of the immediate workstation, more easily interpretable variables were created from the original occupant questionnaires. The individual questionnaire items related to satisfaction with amenities (transportation amenities, biophilia, meeting spaces etc.) were combined into composite variables. The initial assignment of items to thematically-linked groupings was performed based on the judgement of the research team. Principle components analysis (PCA) was then used as a supplementary step to examine the validity of these groupings. This is a well-established technique used in multivariate statistical analysis to bring out strong patterns and groupings in a dataset (Hotelling, 1933). The goal was to bring out thematically similar groupings of the individual questionnaire items to be identified. PCA requires complete data (responses on every item), and this analysis included 
items from every questionnaire module. Therefore, only the cases where occupants opted to complete all the modules were used. For this thesis, PCA was largely used to check the initial assignments of the composite variables to see whether there were any extreme outliers. The final groupings were slightly adjusted based on the PCA results (refer to Appendix C for the PCA table). Cronbach's alpha was used to evaluate the internal consistency (i.e. reliability) of the variable groupings.

Table 25 shows a breakdown of the individual questionnaire items that were grouped together to form new predictors, as well as their Cronbach's alpha values. The value of each composite variable was the mean of the responses to the multiple individual items making up the composite. Most of the composite variables had acceptable internal consistency values, apart from amenities related to private transportation. Nevertheless, this composite was maintained due to the face validity of the grouping. 
Table 25 - Composite amenities satisfaction scales

\section{Variable Individual questionnaire items (satisfaction with)}

\section{(satisfaction with)}

Amenities Related to

Active Transportation

(Cronbach's Alpha $=$

$0.70)$
Supports for your transportation choices: showers

Supports for your transportation choices: storage/locker for personal use

Supports for your transportation choices: storage/locker for bicycles (or other active transportation equipment)

Supports for your transportation choices: maintenance/repair station for bicycles (or other active transportation equipment)

Amenities Related to

Private Transportation

(Cronbach's Alpha $=$

$0.60)$

Supports for your transportation choices: ways to find people with whom to share transportation options

Supports for your transportation choices: availability of parking

Supports for your transportation choices: cost of parking

Supports for your transportation choices: electric vehicle charging stations

Amenities Related to

Public Transportation

(Cronbach's Alpha =

$0.78)$

Supports for your transportation choices: location of public transit stops near your

work destination

Supports for your transportation choices: information about status of public transit

Supports for your transportation choices: availability of public transit at your preferred time

On-Site Amenities

(Cronbach's Alpha $=$ $0.80)$

On-site amenities and their features: daycare

On-site amenities and their features: shopping

On-site amenities and their features: banking

On-site amenities and their features: places to socialize

On-site amenities and their features: places to be calm and contemplative

On-site amenities and their features: artwork in public spaces

On-site amenities and their features: aesthetic appearance of amenity spaces

Outdoor Amenities

(Cronbach's Alpha $=$

$0.84)$

On-site amenities related to the nearby exterior environment: outdoor seating

On-site amenities related to the nearby exterior environment: drinking fountains

On-site amenities related to the nearby exterior environment: pedestrian paths

On-site amenities related to the nearby exterior environment: green space

On-site amenities related to the nearby exterior environment: gathering places

On-site amenities related to the nearby exterior environment: exercise opportunities

Amenities Related to

Sustainability

Availability of programs and design features that support a more sustainable working

(Cronbach's Alpha $=$

$0.75)$

environment

Usability of recycling and composting centres

Distance to waste collection point

Support for reducing paper use

On-site amenities and their features: sustainability information and displays

In-Office Amenities

(Cronbach's Alpha $=$ $0.76)$

IT connectivity and tools to support work

Access to office supplies, materials, and printing facilities

Availability of small meeting rooms

Availability of large meeting rooms

Availability of preferred work locations

Spaciousness of your workspace surroundings

Amenities Related to
Food (Cronbach's Alpha
$=0.88)$

Places to eat and socialize with colleagues

Facilities to store and prepare food

Facilities to wash and store reusable dishes/utensils 
Table 25 (continued).

\begin{tabular}{ll}
\hline $\begin{array}{l}\text { Variable } \\
\text { (satisfaction with) }\end{array}$ & Individual questionnaire items (satisfaction with) \\
\hline $\begin{array}{l}\text { Amenities Related to } \\
\text { Access \& Wayfinding } \\
\text { (Cronbach's Alpha }=\end{array}$ & $\begin{array}{l}\text { Speed and availability of elevators } \\
\text { Access points from the building to the outside } \\
\text { Ability to find your way around inside the building } \\
\text { Ability to locate co-workers when needed } \\
\text { Access to stairs to move between floors }\end{array}$ \\
\hline Amenities Related to & Availability of programs and design features that support a more healthful working \\
environment & \\
(Cronbach's Alpha $=$ & Access to water fountain/bottle refill stations \\
$0.75)$ & Quality of water from water fountain/bottle refill stations \\
& $\begin{array}{l}\text { Natural materials and elements (real or simulated) in the workplace } \\
\text { Cleanliness and maintenance of public spaces }\end{array}$ \\
& $\begin{array}{l}\text { On-site amenities and their features: view of nature in amenity spaces } \\
\text { On-site amenities and their features: fitness }\end{array}$ \\
& On-site amenities and their features: healthy food options \\
\hline
\end{tabular}

\subsection{Descriptive Statistics and Variable Set-up}

Similar to the presentation of the results of the physical workstation conditions, it is important to set the context for the data that is used. The descriptive statistics (means, medians, standard deviations etc.) for the variables used in the regression models were generated for both datasets. Table 26 below shows descriptive statistics for the composite environmental and amenities satisfaction variables. Note that many individual items regarding amenities were in the optional modules of the questionnaire. As such, only a subset of the total sample returned responses to these questions. 
Table 26 - Descriptive statistics for environmental and amenities satisfaction predictor variables (NCR survey sample)

\begin{tabular}{lllllll}
\hline Variable & Mean & Median & Std. Dev. & Min & Max & N \\
\hline SAT_L & 4.9 & 5.2 & 1.3 & 1 & 7 & 1862 \\
SAT_AP & 4.3 & 4.4 & 1.3 & 1 & 7 & 1891 \\
SAT_VT & 4.3 & 4.3 & 1.5 & 1 & 7 & 1889 \\
TRANS_A & 4.1 & 4.0 & 0.8 & 1 & 7 & 1069 \\
TRANS_PR & 3.4 & 3.5 & 0.9 & 1 & 7 & 1060 \\
TRANS_PU & 4.7 & 4.7 & 1.3 & 1 & 7 & 1081 \\
ONSITE & 4.1 & 4.1 & 1.0 & 1 & 7 & 1064 \\
OUTDOOR & 4.4 & 4.5 & 1.2 & 1 & 7 & 1067 \\
SUSTAIN & 4.6 & 4.8 & 1.0 & 1 & 7 & 1080 \\
IN_OFFICE & 4.3 & 4.3 & 1.1 & 1 & 7 & 1783 \\
FOOD & 4.2 & 4.0 & 1.6 & 1 & 7 & 1798 \\
ACCESS & 4.2 & 4.3 & 0.9 & 1 & 7 & 1010 \\
HEALTH & 5.2 & 5.4 & 1.0 & 1 & 7 & 1773 \\
\hline
\end{tabular}

Note: Satisfaction measures on a 7 -point scale, $1=$ very unsatisfied, $4=$ neutral, $7=$ very satisfied.

Table 27 shows descriptive statistics for the outcome variables in this study. These outcome variables were previously-validated composite variables (refer to Table 3 ) and their scales were not identical. Most outcome variables were reported on a 7-point Likert-type scale, though some were not due to the aggregation of questionnaire items or the nature of the questions. For example, "Visual Discomfort" and "Physical Discomfort" were both on a scale from 1 to 25 , where higher values represent more discomfort. "Absence Due to Illness" was reported as the number of occurrences (in days) in the previous month. In general, the descriptive statistics for both predictor and outcome variables indicate an overall state of satisfaction (above the mid-point of the scales), with a few exceptions such as amenities related to private transportation and workplace image. 
Table 27 - Descriptive statistics for outcome variables (NCR survey sample)

\begin{tabular}{lllllll}
\hline Variable & Mean & Median & Std. Dev. & Min & Max & N \\
\hline Overall Environmental Satisfaction (OES) & 4.3 & 4.5 & 1.5 & 1.0 & 7.0 & 1847 \\
Job Satisfaction (JOB_SAT) & 5.3 & 6.0 & 1.4 & 1.0 & 7.0 & 1841 \\
Work Engagement (WRK_ENG) & 5.1 & 5.2 & 1.2 & 1.0 & 7.0 & 1055 \\
$\begin{array}{l}\text { Organizational Commitment (ORG_COM) } \\
\text { Intent to Turnover [higher value means }\end{array}$ & 4.4 & 4.5 & 1.4 & 1.0 & 7.0 & 1113 \\
$\begin{array}{l}\text { more likely to turnover] (TURNOVER) } \\
\text { Workplace Image (WRK_IMG) }\end{array}$ & 3.2 & 3.0 & 1.7 & 1.0 & 7.0 & 1111 \\
$\begin{array}{l}\text { Visual Discomfort [higher value means } \\
\text { more discomfort] (VIS_DIS) }\end{array}$ & 6.9 & 4.0 & 1.5 & 1.0 & 7.0 & 1101 \\
$\begin{array}{l}\text { Physical Discomfort [higher value means } \\
\text { more discomfort] (PHY_DIS) }\end{array}$ & 7.8 & 7.3 & 4.8 & 1.0 & 25.0 & 729 \\
$\begin{array}{l}\text { Absence Due to Illness [\# days in past } \\
\text { month] (ABSENCE) }\end{array}$ & 0.9 & 1.0 & 1.2 & 0.0 & $>5$ & 896 \\
\hline
\end{tabular}

Note: Outcome measures on a 7-point scale, $1=$ very unsatisfied, $4=$ neutral, $7=$ very satisfied.

Table 28 summarizes the coding and setup of the predictor variables related to demographics, workstation characteristics, environmental satisfaction, and amenities satisfaction used in the subsequent analysis. Variable symbols were adopted and shown in the brackets for conciseness in the presentation of results. The NCR dataset includes both private and open-plan style workstations. Approximately one-tenth of the respondents did not have an assigned or permanent work location; these occupants used a workspace reservation system. The unassigned workstations in this study were not randomly distributed amongst the occupants and were only concentrated within a few workgroups. This section of the thesis only uses questionnaire data, so other physical workstation characteristics such as partition height could not be used as predictors. The window variable used in this section is more detailed than what was used previously, as the NCR questionnaire included a more detailed questionnaire item regarding window proximity. 
Table 28 - Description of the predictor variables (NCR survey sample)

\begin{tabular}{|c|c|}
\hline Variable & Description \\
\hline \multirow[t]{5}{*}{ Age } & $18-29(11.4 \%)$ \\
\hline & $20-39(22.5 \%)$ \\
\hline & $40-49(32.1 \%)$ \\
\hline & $50-59(28.4 \%)$ \\
\hline & $>60(5.6 \%)$ \\
\hline \multirow[t]{2}{*}{ Sex } & $0=$ Female $(42.2 \%)$ \\
\hline & $1=$ Male $(57.5 \%)$ \\
\hline \multirow[t]{5}{*}{ Window Location } & $0=$ No window near workstation $(24.6 \%)$ \\
\hline & $1=$ No window in workstation/adjacent workstation, but \\
\hline & window across corridor $(25.6 \%)$ \\
\hline & $2=$ Window in adjacent workstation $(13.6 \%)$ \\
\hline & $3=$ Window in workstation $(36.3 \%)$ \\
\hline \multirow[t]{3}{*}{ Workstation Enclosure } & $0=$ Open workstation, low wall partitions $(21.0 \%)$ \\
\hline & $1=$ Semi-closed workstation, high wall partitions $(68.3 \%)$ \\
\hline & $2=$ Private workstation $(10.7 \%)$ \\
\hline \multirow[t]{2}{*}{ Unassigned WS } & $0=$ Assigned $/$ permanent workstation $(90.7 \%)$ \\
\hline & $1=$ Unassigned workstation $(9.3 \%)$ \\
\hline Satisfaction with Lighting (SAT_L) & Avg of 5 questions: $1=$ very unsatisfied to $7=$ very satisfied \\
\hline Satisfaction with Acoustics and Privacy (SAT_AP) & Avg of 10 questions: $1=$ very unsatisfied to $7=$ very satisfied \\
\hline Satisfaction with Ventilation and Temperature (SAT_VT) & Avg of 3 questions: $1=$ very unsatisfied to $7=$ very satisfied \\
\hline Amenities Related to Active Transportation (TRANS_A) & Avg of 4 questions: $1=$ very unsatisfied to $7=$ very satisfied \\
\hline Amenities Related to Private Transportation (TRANS_PR) & Avg of 4 questions: $1=$ very unsatisfied to $7=$ very satisfied \\
\hline Amenities Related to Public Transportation (TRANS_PU) & Avg of 3 questions: $1=$ very unsatisfied to $7=$ very satisfied \\
\hline On-Site Amenities (ONSITE) & Avg of 7 questions: $1=$ very unsatisfied to $7=$ very satisfied \\
\hline Outdoor Amenities (OUTDOOR) & Avg of 6 questions: $1=$ very unsatisfied to $7=$ very satisfied \\
\hline Amenities Related to Sustainability (SUSTAIN) & Avg of 5 questions: $1=$ very unsatisfied to $7=$ very satisfied \\
\hline In-Office Amenities (IN_OFFICE) & Avg of 6 questions: $1=$ very unsatisfied to $7=$ very satisfied \\
\hline Amenities Related to Food (FOOD) & Avg of 3 questions: $1=$ very unsatisfied to $7=$ very satisfied \\
\hline Amenities Related to Access \& Wayfinding (ACCESS) & Avg of 5 questions: $1=$ very unsatisfied to $7=$ very satisfied \\
\hline Amenities Related to Health \& Well-being (HEALTH) & Avg of 8 questions: $1=$ very unsatisfied to $7=$ very satisfied \\
\hline
\end{tabular}

\subsection{Overall Environmental Satisfaction}

The regression model results of the occupants' overall environmental satisfaction is shown below in Table 29. The results show that older occupants were more satisfied with their overall environment. This is consistent with the findings of some researchers (e.g. Choi et al., 2012; Klitzman \& Stellman, 1989), while others have found conflicting evidence in which older occupants were less satisfied with their workstation environment, specifically with acoustics and privacy (Leder et al., 2016; Newsham et al., 2008). In an earlier section of my analyses of the physical workstation, varying effects of the age of respondents were observed for the different aspects of IEQ. However, the demographic variables did not significantly increase the explained 
variance in the analyses. As such, the influence of age on overall satisfaction should be interpreted with caution.

Table 29 - Overall environmental satisfaction regression

\begin{tabular}{|c|c|c|c|c|c|}
\hline & $\beta$ & $\beta$ & $\beta$ & $\beta$ & Tolerance \\
\hline Age & 0.03 & -0.01 & $0.07 * *$ & $0.06^{*}$ & 0.85 \\
\hline Sex & 0.00 & 0.01 & -0.04 & -0.03 & 0.94 \\
\hline Window Location & & $0.18 * * *$ & -0.02 & -0.01 & 0.73 \\
\hline Workstation Enclosure & & $0.14 * * *$ & -0.01 & 0.00 & 0.81 \\
\hline Unassigned WS & & -0.05 & -0.05 & -0.05 & 0.83 \\
\hline SAT_L & & & $0.17 * * *$ & $0.16^{* * *}$ & 0.40 \\
\hline SAT_AP & & & $0.61 * * *$ & $0.55 * * *$ & 0.39 \\
\hline $\mathrm{SAT}^{-} \mathrm{VT}$ & & & $0.11 * * *$ & $0.08^{*}$ & 0.53 \\
\hline TRANSPO_ACTIVE & & & & -0.03 & 0.78 \\
\hline TRANSPO_PRIV & & & & 0.00 & 0.79 \\
\hline TRANSPO_PUBLIC & & & & -0.05 & 0.78 \\
\hline ONSITE & & & & $0.08 *$ & 0.43 \\
\hline OUTDOOR & & & & $-0.13 * * *$ & 0.46 \\
\hline SUSTAIN & & & & 0.06 & 0.45 \\
\hline IN-OFFICE & & & & 0.06 & 0.33 \\
\hline FOOD & & & & -0.02 & 0.52 \\
\hline HEALTH & & & & $0.10^{*}$ & 0.26 \\
\hline ACCESS & & & & -0.01 & 0.43 \\
\hline $\mathrm{R}^{2}$ Change & 0.00 & $0.06^{* * *}$ & $0.56^{* * *}$ & $0.02 * * *$ & \\
\hline Total $\mathrm{R}^{2}$ & 0.00 & $0.06 * * *$ & $0.62 * * *$ & $0.64 * * *$ & \\
\hline Adjusted $\mathrm{R}^{2}$ & 0.00 & $0.05 * * *$ & $0.61 * * *$ & $0.63 * * *$ & \\
\hline
\end{tabular}

Note: Tolerance values shown are for the last step of the analysis. ${ }^{*} \mathrm{p} \leq 0.05, * * \mathrm{p} \leq 0.01, * * * \mathrm{p} \leq$ $0.001, \mathrm{~N}=632$.

Workstation characteristics such as window proximity and workstation enclosure were shown to have a significant effect on overall satisfaction until the inclusion of the components of environmental satisfaction as predictors. This is likely due to the relationships between workstation characteristics and elements of environmental satisfaction. Variance that might otherwise have been attributed to workstation characteristics is re-allocated to the individual components of environmental satisfaction. This relationship is shown both in prior sections of this thesis as well as other studies (e.g. Veitch et al. 2003). This is further supported by the 
bivariate correlation table (Appendix B). In general, workstation characteristics had a small effect $(6 \%)$ on overall environmental satisfaction.

The regression model showed that all three individual components of environmental satisfaction positively influence overall environmental satisfaction, explaining $56 \%$ of the total variance. This was expected and consistent with Veitch et al. (2007), who previously validated a model highlighting the contributions of individual environmental satisfaction measures to overall environmental satisfaction. Other researchers (e.g. Kim \& de Dear, 2012) have reported similar associations.

Even after controlling for components of environmental satisfaction, satisfaction with amenities factors had a small effect on the outcome (2\%). Satisfaction with health and well-being amenities, which consists of elements such as water fountain access, workplace biophilia, on-site fitness facilities and public space cleanliness, positively influenced overall satisfaction. This result is in agreement with the findings of others (e.g. Bergs, 2002; Foju, 1993), and suggests the that amenities features can have an influence on occupant satisfaction. Satisfaction with outdoor amenities negatively affected overall environmental satisfaction, in the opposite direction to what was expected. It is possible that this is an unintended effect of the regression analysis and the sharing of variance with other predictor variables, highlighting the inter-correlation issues in these analyses. The overall regression model had a large effect size, with $64 \%$ explained variance. This was anticipated, largely due to the inclusion of individual environmental satisfaction measures as predictors in the model and their established relationship with overall environmental satisfaction. 


\subsection{Job Satisfaction}

The regression results for job satisfaction is shown below in Table 30. The model did not show age and sex to have a significant effect on job satisfaction. Like the overall environmental satisfaction results, window proximity had an effect on job satisfaction until the inclusion of the individual environmental satisfaction measures. The results generally show that demographic factors and workstation characteristics had a limited influence on job satisfaction.

Table 30 - Job satisfaction regression

\begin{tabular}{lccccc}
\hline & $\boldsymbol{\beta}$ & $\boldsymbol{\beta}$ & $\boldsymbol{\beta}$ & $\boldsymbol{\beta}$ & Tolerance \\
\hline Age & 0.01 & -0.01 & 0.04 & 0.02 & 0.85 \\
Sex & -0.01 & 0.00 & -0.04 & -0.02 & 0.94 \\
\hline Window Location & & $0.09^{*}$ & -0.07 & -0.04 & 0.73 \\
Workstation Enclosure & & 0.05 & -0.04 & -0.01 & 0.81 \\
Unassigned WS & & -0.03 & -0.02 & -0.01 & 0.83 \\
\hline SAT_L & & & $0.17^{* *}$ & $0.14^{* *}$ & 0.40 \\
SAT_AP & & & $0.37^{* * *}$ & $0.28^{* * *}$ & 0.39 \\
SAT_VT & & & 0.03 & -0.02 & 0.53 \\
\hline TRANSPO_ACTIVE & & & & -0.04 & 0.78 \\
TRANSPO_PRIV & & & & -0.03 & 0.79 \\
TRANSPO_PUBLIC & & & & -0.07 & 0.78 \\
ONSITE & & & & -0.019 & 0.43 \\
OUTDOOR & & & & 0.09 & 0.46 \\
SUSTAIN & & & & 0.03 & 0.33 \\
IN-OFFICE & & & & -0.01 & 0.52 \\
FOOD & & & & $0.14 *$ & 0.26 \\
HEALTH & & & & 0.07 & 0.42 \\
ACCESS & & & & & \\
\hline R $^{2}$ Change & 0.00 & 0.01 & $0.23^{* * *}$ & $0.03^{* *}$ & \\
Total R ${ }^{2}$ & 0.00 & 0.01 & $0.24^{* * *}$ & $0.27^{* * *}$ & \\
Adjusted R & 0.00 & 0.00 & $0.23^{* * *}$ & $0.25^{* * *}$ & \\
\hline
\end{tabular}

Note: Tolerance values shown are for the last step of the analysis. ${ }^{*} \mathrm{p} \leq 0.05,{ }^{* *} \mathrm{p} \leq 0.01,{ }^{* * *} \mathrm{p} \leq$ $0.001, \mathrm{~N}=631$.

The job satisfaction model also showed that components of environmental satisfaction had a significant effect on job satisfaction, explaining $23 \%$ of the total variance. However, 
satisfaction with ventilation and temperature did not significantly predict job satisfaction. This observation is interesting for several reasons. It prompts the discussion of relative importance of the components of environmental satisfaction. Many green building standards (e.g. LEED) and field studies of buildings (e.g. Ali, Chua, \& Lim, 2015; Pei, Lin, Liu, \& Zhu, 2015) focus on the thermal comfort aspects of IEQ. The results here suggest that other aspects of the indoor environment, such as acoustics, play a much larger role in predicting job satisfaction and overall environmental satisfaction than temperature. Newsham et al. (2009) have reported similar results, highlighting the importance of acoustics and privacy on job satisfaction. Given the demonstrated effects of acoustics on occupants, perhaps practitioners and researchers should place more emphasis on the acoustics aspect of IEQ.

In this model, satisfaction with amenities variables had a small effect on job satisfaction $(3 \%)$, with satisfaction with health and well-being amenities being a statistically significant predictor variable. It was expected that the workstation environment would explain more variance than the amenities factors, given most of an occupant's working day is spent at their workstation. In fact, it is notable that amenities explained any significant amount of variance in the model. Again, it was health and well-being amenities that positively affected job satisfaction. The overall regression model had a large effect on the outcome (27\%), further highlighting the importance of environmental and amenities factors on job satisfaction.

\subsection{Work Engagement}

The regression results for work engagement is shown below in Table 31. The regression model shows that sex had a small effect (1\%), with females more engaged than their male counterparts. This result is surprising, as prior studies have demonstrated that females were more dissatisfied with the physical work environment (Choi et al., 2012; Leder et al., 2016; Newsham 
et al., 2008; Yildirim et al., 2007). This dissatisfaction was expected to result in lower engagement, however this was not observed.

Table 31 - Work engagement regression

\begin{tabular}{|c|c|c|c|c|c|}
\hline & $\beta$ & $\beta$ & $\boldsymbol{\beta}$ & $\beta$ & Tolerance \\
\hline Age & 0.05 & 0.05 & 0.07 & 0.05 & 0.85 \\
\hline Sex & $-0.10 * *$ & $-0.11 * *$ & $-0.13 * *$ & $-0.10 * *$ & 0.93 \\
\hline Window Location & & 0.04 & -0.01 & 0.01 & 0.73 \\
\hline Workstation Enclosure & & 0.04 & 0.01 & 0.04 & 0.82 \\
\hline Unassigned WS & & $0.09 *$ & $0.09 *$ & $0.10^{*}$ & 0.83 \\
\hline SAT_L & & & 0.03 & 0.00 & 0.39 \\
\hline SAT_AP & & & $0.16^{* *}$ & 0.09 & 0.39 \\
\hline $\mathrm{SAT}^{-} \mathrm{VT}$ & & & 0.06 & 0.00 & 0.52 \\
\hline TRANSPO_ACTIVE & & & & 0.06 & 0.77 \\
\hline TRANSPO_PRIV & & & & 0.06 & 0.79 \\
\hline TRANSPO_PUBLIC & & & & 0.04 & 0.79 \\
\hline ONSITE & & & & -0.06 & 0.43 \\
\hline OUTDOOR & & & & 0.04 & 0.47 \\
\hline SUSTAIN & & & & 0.05 & 0.45 \\
\hline IN-OFFICE & & & & -0.02 & 0.33 \\
\hline FOOD & & & & -0.09 & 0.52 \\
\hline HEALTH & & & & $0.18 *$ & 0.26 \\
\hline ACCESS & & & & 0.04 & 0.42 \\
\hline $\mathrm{R}^{2}$ Change & $0.01 *$ & 0.01 & $0.05 * * *$ & $0.05 * * *$ & \\
\hline Total $\mathrm{R}^{2}$ & $0.01 *$ & $0.02 *$ & $0.07 * * *$ & $0.11 * * *$ & \\
\hline Adjusted $\mathrm{R}^{2}$ & $0.01 *$ & $0.01 *$ & $0.06^{* * *}$ & $0.09 * * *$ & \\
\hline
\end{tabular}

Note: Tolerance values shown are for the last step of the analysis. ${ }^{*} \mathrm{p} \leq 0.05,{ }^{*} \mathrm{p} \leq$ $0.01, * * * \mathrm{p} \leq 0.001, \mathrm{~N}=613$.

Being in an unassigned workstation also positively affected work engagement. This is broadly in agreement with the findings of Robertson, Huang, O’Neill, \& Schleifer (2008), who reported similar beneficial effects of flexible working on communication and overall feeling of the workspace. However, workstation characteristics did not significantly increase the explained variance. As such, the positive influence of flexible workstations should be interpreted with caution, particularly considering that unassigned workstations did not affect any other outcome. 
The unassigned workstations were located within a few specific workgroups, so this observation may be due to workgroup differences rather than the workstations. The host organization's design intent is to transition most staff to unassigned workstations. Therefore, the effects of this mode of office accommodation on satisfaction, well-being, and organizational productivity is worthy of further study.

Satisfaction with individual components of workstation environment had a small effect $\left(\mathrm{R}^{2}=5 \%\right)$, with acoustics and privacy being a statistically significant predictor variable before the inclusion of the amenity factors. Lighting, as well as ventilation and temperature, were not found to have an effect on the outcome.

Again, satisfaction with health and well-being amenities had an effect on the outcome variable, with amenities factors accounting for $5 \%$ of the explained variance. This observed effect is comparable to the environmental satisfaction variables, pointing to the importance of amenities outside of the immediate workstation. Many factors outside of those included in this study can be expected to influence work engagement, and the medium effect size (11\%) predicted by this model is noteworthy. It indicates that amenities and workstation environment can influence engagement, suggesting that building practitioners should consider these factors when looking to improve organizational productivity.

\subsection{Organizational Commitment}

The regression results for organizational commitment is shown below in Table 32 .

Similar to the results of work engagement, the organizational commitment regression suggests that females were more committed than their male counterparts. This effect disappears with the inclusion of amenities variables as predictors. Furthermore, the variance explained by 
demographic factors was not statistically significant. This suggests that the observed differences in organizational commitment between the sexes are marginal.

Table 32 - Organizational commitment regression

\begin{tabular}{|c|c|c|c|c|c|}
\hline & $\beta$ & $\beta$ & $\beta$ & $\beta$ & Tolerance \\
\hline Age & 0.01 & 0.00 & 0.04 & 0.02 & 0.85 \\
\hline Sex & $-0.08 *$ & $-0.09 *$ & $-0.10^{*}$ & -0.07 & 0.94 \\
\hline Window Location & & 0.07 & 0.04 & 0.07 & 0.73 \\
\hline Workstation Enclosure & & 0.04 & -0.02 & 0.01 & 0.81 \\
\hline Unassigned WS & & 0.06 & 0.06 & 0.06 & 0.83 \\
\hline SAT_L & & & -0.08 & $-0.12 *$ & 0.40 \\
\hline SAT_AP & & & $0.26^{*}$ & $0.16^{* *}$ & 0.39 \\
\hline SAT VT & & & 0.08 & 0.00 & 0.53 \\
\hline TRANSPO_ACTIVE & & & & 0.02 & 0.78 \\
\hline TRANSPO_PRIV & & & & 0.03 & 0.79 \\
\hline TRANSPO_PUBLIC & & & & 0.03 & 0.78 \\
\hline ONSITE & & & & -0.07 & 0.43 \\
\hline OUTDOOR & & & & -0.03 & 0.46 \\
\hline SUSTAIN & & & & 0.02 & 0.45 \\
\hline IN-OFFICE & & & & 0.04 & 0.33 \\
\hline FOOD & & & & -0.07 & 0.52 \\
\hline HEALTH & & & & $0.34 * * *$ & 0.26 \\
\hline ACCESS & & & & 0.02 & 0.43 \\
\hline $\mathrm{R}^{2}$ Change & 0.01 & 0.01 & $0.07^{* * * *}$ & $0.07 * * *$ & \\
\hline Total $\mathrm{R}^{2}$ & 0.01 & 0.02 & $0.08 * * *$ & $0.15^{* * *}$ & \\
\hline Adjusted $\mathrm{R}^{2}$ & 0.00 & 0.01 & $0.07 * * *$ & $0.12 * * *$ & \\
\hline
\end{tabular}

Note: Tolerance values shown are for the last step of the analysis. ${ }^{*} \mathrm{p} \leq 0.05,{ }^{* *} \mathrm{p} \leq$ $0.01, * * * \mathrm{p} \leq 0.001, \mathrm{~N}=632$.

The individual components of environmental satisfaction had a small effect (7\%) on organizational commitment. It is unclear why lighting had a negative influence on commitment. Logically, it does not make sense that occupants who are less satisfied with lighting are less committed to their organizations. However, based on the fact that it was not a statistically significant predictor until the inclusion of the amenities variables (last step of the model), it could be a result of intercorrelation within the predictors and mis-partitioning due to the 
inclusion of these predictors. The results again highlight the effects of acoustics and privacy, and further highlights the importance of acoustics and privacy as a key aspect of IEQ. As previously mentioned, high performance buildings often look to thermal performance as a key performance indicator. The results here reinforce the importance of acoustics and privacy; organizations wishing to improve performance should consider focussing on improving this aspect of IEQ.

Satisfaction with amenities variables accounted for $7 \%$ of the variance, same as the environmental satisfaction factors. This further suggests that the influence of building amenities can be comparable to that of the workstation conditions. As such, there should be more focus on the factors outside of the immediate workstation. Of the amenity factors, satisfaction with health and well-being amenities had a positive effect on organizational commitment, highlighting the benefits of features such as biophilia and public space cleanliness. Similar to work engagement, many factors outside of those considered in this analysis can be expected to influence organizational commitment. For instance, specific job duties, salary and benefits, as well as the team relations can all influence organizational commitment. Therefore, a medium effect size (15\%) explained by the workstation environment and amenities satisfaction variables is notable.

\subsection{Turnover Intent}

The regression results for intent to turnover is shown below in Table 33. Sex was shown have a small but statistically significant effect on turnover intent, with males more with higher intent than females. This reinforces the previous analyses results, in which females were found to be more engaged and committed to their work. No workstation characteristics were found to be statistically significant predictors of turnover intent. 
Table 33 - Turnover intent regression

\begin{tabular}{|c|c|c|c|c|c|}
\hline & $\boldsymbol{\beta}$ & $\boldsymbol{\beta}$ & $\boldsymbol{\beta}$ & $\beta$ & Tolerance \\
\hline Age & 0.03 & 0.04 & 0.00 & 0.01 & 0.85 \\
\hline Sex & $0.10^{* *}$ & $0.10^{*}$ & $0.11^{* *}$ & $0.10^{*}$ & 0.94 \\
\hline Window Location & & -0.04 & 0.00 & -0.01 & 0.73 \\
\hline Workstation Enclosure & & -0.03 & 0.02 & 0.01 & 0.81 \\
\hline Unassigned WS & & 0.00 & -0.01 & -0.02 & 0.83 \\
\hline SAT_L & & & 0.03 & 0.06 & 0.40 \\
\hline SAT_AP & & & $-0.24 * * *$ & $-0.21 * * *$ & 0.39 \\
\hline SAT_VT & & & -0.03 & 0.01 & 0.53 \\
\hline TRANSPO_ACTIVE & & & & -0.02 & 0.78 \\
\hline TRANSPO_PRIV & & & & -0.01 & 0.79 \\
\hline TRANSPO_PUBLIC & & & & 0.03 & 0.78 \\
\hline ONSITE & & & & 0.04 & 0.43 \\
\hline OUTDOOR & & & & -0.05 & 0.46 \\
\hline SUSTAIN & & & & 0.00 & 0.45 \\
\hline IN-OFFICE & & & & 0.06 & 0.33 \\
\hline FOOD & & & & 0.06 & 0.52 \\
\hline HEALTH & & & & $-0.19 *$ & 0.26 \\
\hline ACCESS & & & & -0.08 & 0.43 \\
\hline $\mathrm{R}^{2}$ Change & $0.01 *$ & 0.00 & $0.05^{* * *}$ & $0.03^{*}$ & \\
\hline Total $\mathrm{R}^{2}$ & $0.01 *$ & 0.01 & $0.07 * * *$ & $0.10 * * *$ & \\
\hline Adjusted $\mathrm{R}^{2}$ & $0.01 *$ & 0.01 & $0.06^{* * *}$ & $0.07 * * *$ & \\
\hline
\end{tabular}

Note: Tolerance values shown are for the last step of the analysis. ${ }^{*} \mathrm{p} \leq 0.05,{ }^{* *} \mathrm{p} \leq$ $0.01,{ }^{* * *} \mathrm{p} \leq 0.001, \mathrm{~N}=632$.

Workstation environmental satisfaction was found to explain $7 \%$ of the variance, which is a small effect size. Of the environmental satisfaction predictors, acoustics and privacy was found to influence turnover intent; people who were more content with their acoustic environment had less intent to turnover.

The regression model also showed satisfaction with health and well-being amenities to influence turnover intent. Overall, $10 \%$ of the variance was explained, which is a medium effect size. Many unobserved external factors can be expected to influence turnover, like specific job duties and team relations, as well as contract or term workers who will be looking for a new 
position regardless of other factors. Thus, although the effect size of this model is not large, it was striking that any predictors were statistically significant. The results again suggest the importance of traditionally overlooked factors like satisfaction with acoustics and privacy and health and well-being amenities on employee retention. These influencing factors can be a tool for organizations to address possible sources of employee turnover and reduced productivity.

\subsection{Workplace Image}

The regression results for workplace image is shown below in Table 34. Demographic factors were not found to have a significant effect on workplace image. More workstation enclosure negatively influenced workplace image, though its explained variance was not statistically significant. The enclosed offices were also most likely not to have been renovated in decades, confounding time with enclosure. 
Table 34 - Workplace image regression

\begin{tabular}{|c|c|c|c|c|c|}
\hline & $\beta$ & $\boldsymbol{\beta}$ & $\boldsymbol{\beta}$ & $\boldsymbol{\beta}$ & Tolerance \\
\hline Age & 0.01 & 0.00 & 0.05 & -0.01 & 0.85 \\
\hline Sex & 0.01 & 0.01 & -0.04 & 0.01 & 0.94 \\
\hline Window Location & & 0.07 & -0.04 & 0.01 & 0.73 \\
\hline Workstation Enclosure & & -0.04 & $-0.14 * * *$ & $-0.10^{* *}$ & 0.81 \\
\hline Unassigned WS & & -0.04 & -0.06 & -0.06 & 0.83 \\
\hline SAT_L & & & 0.02 & -0.03 & 0.40 \\
\hline SAT_AP & & & $0.37 * * *$ & $0.17 * * *$ & 0.39 \\
\hline SATVT & & & $0.25^{* * *}$ & $0.14 * * *$ & 0.53 \\
\hline TRANSPO_ACTIVE & & & & -0.02 & 0.78 \\
\hline TRANSPO_PRIV & & & & 0.05 & 0.79 \\
\hline TRANSPO_PUBLIC & & & & -0.03 & 0.78 \\
\hline ONSITE ${ }^{-}$ & & & & $0.18^{* * *}$ & 0.43 \\
\hline OUTDOOR & & & & -0.08 & 0.46 \\
\hline SUSTAIN & & & & 0.07 & 0.45 \\
\hline IN-OFFICE & & & & $0.11 *$ & 0.33 \\
\hline FOOD & & & & 0.03 & 0.52 \\
\hline HEALTH & & & & $0.22 * * *$ & 0.26 \\
\hline ACCESS & & & & 0.01 & 0.42 \\
\hline $\mathrm{R}^{2}$ Change & 0.00 & 0.01 & $0.29 * * *$ & $0.12 * * *$ & \\
\hline Total $\mathrm{R}^{2}$ & 0.00 & 0.01 & $0.30^{* * *}$ & $0.43 * * *$ & \\
\hline Adjusted $\mathrm{R}^{2}$ & 0.00 & 0.00 & $0.29 * * *$ & $0.41^{* * *}$ & \\
\hline
\end{tabular}

Note: Tolerance values shown are for the last step of the analysis. ${ }^{*} \mathrm{p} \leq 0.05,{ }^{* *} \mathrm{p} \leq$ $0.01, * * * \mathrm{p} \leq 0.001, \mathrm{~N}=633$.

Components of environmental satisfaction (i.e. acoustics and privacy, ventilation and temperature) were positive predictors of workplace image and explained $29 \%$ of the total variance; this is an expected and logical result. Occupants who perceive less noise, distractions, and have comfortable thermal environments are expected to view the workplace in a more positive light.

Satisfaction with on-site amenities, which consist of on-site shopping, banking, and places to socialize, as well as satisfaction with health and well-being amenities had an effect on workplace image. The amenities variables had a medium effect on workplace image, accounting 
for $12 \%$ of the total variance. While amenities satisfaction had less explained variance than workstation environment, it still contributed meaningfully. Overall, a large effect size (43\%) was observed in the workplace image analysis. The significant effects of both satisfaction with the workstation environment and amenities suggest that organizations should look to these factors to improve corporate image.

\subsection{Visual Discomfort}

The regression results for visual discomfort is shown below in Table 35. Sex was a statistically significant predictor in this model, accounting for $2 \%$ of the total variance (small effect). Females were prone to more visual discomfort, in agreement with previous studies (e.g. Aries, Veitch, \& Newsham, 2010; Thayer et al., 2010). A possible explanation is that women might be more likely than men to report discomfort. It was expected for this discomfort to result in less work engagement and less organizational commitment in females, and consequently more turnover intent, but the reverse was observed. As previously noted, this could be due to the many other unseen contributors to outcome variables like engagement, commitment, and turnover intent. 
Table 35 - Visual discomfort regression

\begin{tabular}{lccccc}
\hline & $\boldsymbol{\beta}$ & $\boldsymbol{\beta}$ & $\boldsymbol{\beta}$ & $\boldsymbol{\beta}$ & Tolerance \\
\hline Age & 0.01 & 0.03 & 0.02 & 0.02 & 0.86 \\
Sex & $-0.15^{* *}$ & $-0.15^{* *}$ & $-0.11^{*}$ & $-0.10^{*}$ & 0.94 \\
\hline Window Location & & $-0.10^{*}$ & 0.00 & 0.00 & 0.70 \\
Workstation Enclosure & & -0.01 & 0.05 & 0.06 & 0.77 \\
Unassigned WS & & -0.05 & -0.03 & -0.03 & 0.85 \\
\hline SAT_L & & & $-0.14^{*}$ & $-0.15^{*}$ & 0.38 \\
SAT_AP & & & $-0.18^{* *}$ & $-0.17^{*}$ & 0.39 \\
SAT_VT & & & $-0.18^{* *}$ & $-0.16^{* *}$ & 0.55 \\
\hline TRANSPO_ACTIVE & & & & -0.06 & 0.81 \\
TRANSPO_PRIV & & & & $-0.11^{*}$ & 0.83 \\
TRANSPO_PUBLIC & & & & -0.03 & 0.80 \\
ONSITE & & & & 0.03 & 0.42 \\
OUTDOOR & & & & 0.10 & 0.47 \\
SUSTAIN & & & & 0.02 & 0.45 \\
IN-OFFICE & & & & 0.02 & 0.50 \\
FOOD & & & & -0.16 & 0.27 \\
HEALTH & & & & 0.00 & 0.44 \\
ACCESS & & & & & \\
\hline R $^{2}$ Change & & & & & \\
Total R & & & & & \\
Adjusted R & & 0.01 & $0.16^{* * *}$ & 0.03 & \\
\hline Not Tol & $0.02^{* *}$ & $0.03^{*}$ & $0.20^{* * *}$ & $0.23^{* * *}$ & \\
\hline
\end{tabular}

Note: Tolerance values shown are for the last step of the analysis. ${ }^{*} \mathrm{p} \leq 0.05,{ }^{* *} \mathrm{p} \leq$ $0.01, * * * \mathrm{p} \leq 0.001, \mathrm{~N}=426$.

All three components of environmental satisfaction (lighting, acoustics and privacy, ventilation and temperature) had positive effects on reducing visual discomfort, explaining $16 \%$ of the total variance. It was expected that lighting and ventilation factors would influence visual discomfort, but acoustics and privacy having an influence was intriguing. It is possible that this finding was the result of intercorrelation and relationships between the components of environmental satisfaction (Veitch et al., 2007), but it again highlights the influence of office acoustics. 
Satisfaction with private transportation amenities was also found to be statistically significant, although the change in variance was not. This suggests that while private transportation amenities could be influential, satisfaction with this feature has a minimal effect on visual discomfort experienced at work. The visual discomfort regression showed an overall explained variance of $23 \%$.

\subsection{Physical Discomfort}

The regression results for physical discomfort is shown below in Table 36. In general, the physical discomfort results were very similar to that of visual discomfort. Again, sex influenced physical discomfort, with females experiencing more physical symptoms than males. Window proximity was shown to have a positive effect in decreasing physical discomfort. However, this effect disappeared upon the inclusion of environmental satisfaction measures. The change in variance due to workstation characteristics was not significant, suggesting that the benefits of window proximity on discomfort are limited. 
Table 36 - Physical discomfort regression

\begin{tabular}{|c|c|c|c|c|c|}
\hline & $\boldsymbol{\beta}$ & $\beta$ & $\boldsymbol{\beta}$ & $\boldsymbol{\beta}$ & Tolerance \\
\hline Age & 0.07 & 0.09 & 0.05 & 0.06 & 0.85 \\
\hline Sex & $-0.17 * * *$ & $-0.17 * * *$ & $-0.13 * *$ & $-0.14 * *$ & 0.93 \\
\hline Window Location & & $-0.10^{*}$ & -0.05 & -0.06 & 0.70 \\
\hline Workstation Enclosure & & -0.01 & 0.07 & 0.03 & 0.75 \\
\hline Unassigned WS & & -0.07 & -0.03 & -0.04 & 0.82 \\
\hline SAT L & & & 0.02 & 0.03 & 0.39 \\
\hline SAT_AP & & & $-0.20 * * *$ & $-0.20 * *$ & 0.40 \\
\hline SAT VT & & & $-0.27 * * *$ & $-0.27 * * *$ & 0.52 \\
\hline TRANSPO_ACTIVE & & & & $-0.14^{* *}$ & 0.79 \\
\hline TRANSPO_PRIV & & & & $-0.15 * * *$ & 0.82 \\
\hline TRANSPO_PUBLIC & & & & -0.06 & 0.76 \\
\hline ONSITE & & & & $0.16^{*}$ & 0.42 \\
\hline OUTDOOR & & & & -0.10 & 0.48 \\
\hline SUSTAIN & & & & -0.06 & 0.44 \\
\hline IN-OFFICE & & & & 0.02 & 0.34 \\
\hline FOOD & & & & 0.01 & 0.52 \\
\hline HEALTH & & & & 0.05 & 0.26 \\
\hline ACCESS & & & & 0.04 & 0.44 \\
\hline $\mathrm{R}^{2}$ Change & $0.03 * * *$ & 0.01 & $0.15^{* * *}$ & $0.06^{* * * *}$ & \\
\hline Total $\mathrm{R}^{2}$ & $0.03 * * *$ & $0.05 * * *$ & $0.20^{* * *}$ & $0.26 * * *$ & \\
\hline Adjusted $\mathrm{R}^{2}$ & $0.03 * * *$ & $0.04 * * *$ & $0.18^{* * *}$ & $0.23 * * *$ & \\
\hline
\end{tabular}

Note: Tolerance values shown are for the last step of the analysis. ${ }^{*} \mathrm{p} \leq 0.05,{ }^{* *} \mathrm{p} \leq$ $0.01, * * * \mathrm{p} \leq 0.001, \mathrm{~N}=422$.

Various satisfaction with amenities variables were shown to have an effect on physical discomfort. Satisfaction with active transportation amenities significantly reduced physical discomfort. This makes logical sense, as occupants are more likely to engage in active modes of transportation (e.g. biking, walking) if they are satisfied with the amenities to accommodate the decision. While the significance of private transportation amenities does not immediately align with common sense, there are some possible explanations. Occupants who are more satisfied with parking and other private transportation amenities are likely to experience reduced levels of stress when driving and parking their vehicles. The reduced stress could indirectly contribute to the observed benefits to visual and physical discomfort. Higher satisfaction with on-site 
amenities increased physical discomfort to a degree. It is not immediately clear why this result was observed, but it could be a limitation in the analysis and a consequence of shared variance in the regression model. Surprisingly, health and well-being satisfaction did not significantly predict physical discomfort. This could be due to other unaccounted factors such as office equipment ergonomics, which has been shown to affect physical comfort (Klitzman \& Stellman, 1989). Additionally, the reported levels of physical and visual discomfort were generally low, so it is possible that there was little correlation to explain.

The overall model showed a large effect size (26\%), suggesting that workstation environment and amenities satisfaction can influence physical discomfort to a reasonable degree. The considerable effect sizes observed in both the visual and physical discomfort analyses point to the importance of these elements. Workplace decision makes should look to improve upon these factors to reduce discomfort and enhance occupant health and well-being.

\subsection{Absences}

The regression results for absences due to illness is shown below in Table 37. The regression analysis shows satisfaction with acoustics and privacy reduced absenteeism, though the change in explained variance was not significant. Furthermore, it was not statistically significant until the inclusion of the amenity variables into the model. Still, this observation suggests that more focus should be placed on acoustics and privacy, an often-overlooked aspect of IEQ in the green building movement.

Satisfaction with public transportation and on-site amenities were associated with increased absenteeism, with a small observed effect size. A possible explanation for these

unexpected results is that occupants generally reported low levels of absences (mean value of 0.9 days absent for illness in the past month), wherein small differences in the sample could have 
skewed the regression model. Based on the small effect size and the low levels of reported absenteeism in the dataset, the results do not convincingly indicate that satisfaction with workstation environment or amenities have a large influence on absenteeism due to sicknesses.

Table 37 - Absences due to illness regression

\begin{tabular}{|c|c|c|c|c|c|}
\hline & $\boldsymbol{\beta}$ & $\boldsymbol{\beta}$ & $\beta$ & $\boldsymbol{\beta}$ & Tolerance \\
\hline Age & 0.06 & 0.07 & 0.05 & 0.05 & 0.85 \\
\hline Sex & -0.06 & -0.06 & -0.05 & -0.06 & 0.94 \\
\hline Window Location & & -0.07 & -0.08 & -0.07 & 0.72 \\
\hline Workstation Enclosure & & 0.01 & 0.04 & 0.04 & 0.80 \\
\hline Unassigned WS & & 0.00 & 0.00 & -0.01 & 0.85 \\
\hline SAT_L & & & 0.08 & 0.06 & 0.42 \\
\hline SAT_AP & & & -0.10 & $-0.13 *$ & 0.42 \\
\hline $\mathrm{SAT}^{-} \mathrm{VT}$ & & & -0.07 & -0.08 & 0.57 \\
\hline TRANSPO_ACTIVE & & & & -0.03 & 0.76 \\
\hline TRANSPO_PRIV & & & & -0.02 & 0.78 \\
\hline TRANSPO_PUBLIC & & & & $0.11^{*}$ & 0.79 \\
\hline ONSITE & & & & $0.17^{*}$ & 0.43 \\
\hline OUTDOOR & & & & 0.05 & 0.46 \\
\hline SUSTAIN & & & & -0.12 & 0.46 \\
\hline IN-OFFICE & & & & 0.02 & 0.34 \\
\hline FOOD & & & & -0.04 & 0.51 \\
\hline HEALTH & & & & -0.11 & 0.26 \\
\hline ACCESS & & & & 0.12 & 0.45 \\
\hline $\mathrm{R}^{2}$ Change & 0.01 & 0.00 & 0.01 & $0.04 *$ & \\
\hline Total $\mathrm{R}^{2}$ & 0.01 & 0.01 & 0.02 & $0.06^{*}$ & \\
\hline Adjusted $\mathrm{R}^{2}$ & 0.00 & 0.00 & 0.01 & $0.03^{*}$ & \\
\hline
\end{tabular}

Note: Tolerance values shown are for the last step of the analysis. ${ }^{*} \mathrm{p} \leq 0.05,{ }^{*} \mathrm{p} \leq$ $0.01, * * * \mathrm{p} \leq 0.001, \mathrm{~N}=508$.

\subsection{Discussion - Broader Amenities}

While the regression results in the previous sections have shown significant effects of amenities and workstation environment variables on occupant satisfaction and organizational productivity, there is value in discussing the predictor variables that were not found to be statistically significant. Surprisingly, proximity to windows was not shown to have a significant 
effect in any of the final regression models. While the benefits of natural daylighting and access to outside views have been well documented in the literature (Aries et al., 2010; Elzeyadi, 2011; Farley \& Veitch, 2001; Frontczak et al., 2012; Galasiu \& Veitch, 2006; Leder et al., 2016; Yildirim et al., 2007), and even confirmed in the analysis of the workstation environment in previous sections, this was not observed here. This was briefly discussed previously, and can largely be attributed to the link between window location and lighting satisfaction. Satisfaction with lighting was included as a separate predictor in these regression models, accounting for variance that might otherwise have been attributed to presence of windows. The hierarchical regression tables illustrate this result, where window location consistently predicted the outcome variables prior to the inclusion of lighting satisfaction. This is further supported by the bivariate correlation table (Appendix B), where significant correlations between window location and lighting satisfaction were observed.

The results of the analyses also unable to replicate the beneficial effects of increased enclosure on environmental satisfaction (Frontczak et al. 2012; Kim and de Dear 2013; Newsham et al. 2008; Pejtersen et al. 2006) and organizational productivity measures (Brill et al., 2001; De Croon et al. 2005; Leder et al. 2016; Lee 2010; Otterbring et al. 2018). The cause is likely the inclusion of satisfaction with acoustics and privacy and satisfaction with ventilation and temperature as separate predictors. Like the observed relationship between window location and lighting satisfaction, the bivariate correlation table shows clear correlations between workstation enclosure and satisfaction with acoustics and privacy (refer to Appendix B). In the previous sections, the results showed similar correlations of partition height and enclosed workstations to components of environmental satisfaction (acoustics and privacy, ventilation and temperature). 
While the results of the hierarchical regression analysis show some predictor variables to be statistically significant in the opposite direction to what was expected, the overall trends and collection of effects across the multiple analyses is of greater value. In general, satisfaction with health and well-being amenities to have a consistent positive effect on the outcome variables, even after controlling for other relevant predictor variables. These amenities, composed of elements such as quality of and access to water fountains, biophilia in the office, on-site fitness facilities, and cleanliness of public spaces were shown to influence overall satisfaction and several organizational productivity indices. Prior research found similar beneficial effects of elements such as biophilia (Al Horr et al., 2017; Bergs, 2002; Gray \& Birrell, 2014; Kamarulzaman, Saleh, Hashim, Hashim, \& Abdul-Ghani, 2011; MacKerron \& Mourato, 2013) and on-site fitness facilities (Shephard, 1992). Others studies also reported on the positive influences of the cleanliness and maintenance of public spaces (Foju 1993; Lee 2010; World Green Building Council 2014). Surprisingly, health and well-being satisfaction did not significantly predict physical discomfort. This could be due to other unaccounted factors such as office equipment ergonomics, which has been shown to significantly affect physical comfort (Klitzman \& Stellman, 1989). Additionally, the reported levels of physical and visual discomfort were generally low, so it is possible that there was little correlation to explain.

Research has shown that people are costly, with staffing making up $90 \%$ of a typical business' operation costs (World Green Building Council, 2014). Yet, in the current sustainable building movement, priority is often given to reducing energy consumption and costs. These results suggest that amenities factors can have positive effects on the organizational productivity and satisfaction. Even though the observed effect sizes of some of these outcomes are small, improvements to these amenities can potentially have a larger influence on organizational costs 
than the building operations. These findings align with the WELL building scheme, which highlights factors contributing to a more healthful workplace for the occupants. It takes into consideration the building and site amenities, addressing topics such as drinking water, food facilities, biophilia and more (International WELL Building Institute, 2014). Researchers should be cognisant of this when performing post-occupancy evaluation research in WELL certified buildings.

In the interpretation of these results, it should be noted that the participants of the NCR study were located at the same site. Although there were variations in the local office features such as meeting rooms and kitchens, occupants reported on the same site amenities like parking and transit access. While this is a drawback in some ways, there are also benefits to single building studies over studies of multiple buildings/organizations. The results are more consistent and there is less noise in data gathered from a single organization with varying working conditions.

This study also only evaluated the direct effects of the predictors on the outcome variables, but not possible indirect effects. Other modelling approaches (e.g. structural equation modelling) could be used in future studies to evaluate the indirect influences of these variables. It should be reiterated that this study used measures of amenities satisfaction as predictors rather than their presence. These satisfaction measures are not necessarily indicative of the influence of specific amenity features physically provided to occupants. As such, future research is necessary to investigate the effects of the presence of various building amenities.

Nonetheless, the results suggest that occupant perceptions of building amenities, particularly those related to health and well-being, can influence satisfaction and organizational productivity. As such, future building researchers should consider the measurement of amenities 
along with typical workstation conditions. Designers and office planners should also take into account the influence and benefits of communal amenity features in their works, and not only focus on the immediate workstation hardware or layout. 


\section{Conclusions and Recommendations}

The overall research objective of this thesis was to examine the influence of various aspects of the physical built environments on occupants in an office setting. Various conclusions can be drawn based on the analyses and are summarized in this section in order of the original research questions.

\subsection{Research Question \#1}

The first part of this thesis aimed to examine the physical workstation conditions and various aspects of the indoor environment in relation to occupant satisfaction. The most obvious result of the lighting analyses was that window proximity had a consistent positive influence on lighting satisfaction, even after taking into consideration illuminance measurements. This suggests that the view and presence of natural light play a role on satisfaction, and that designers should focus on getting occupants closer to windows. The benefit of higher measured desktop illuminance was also partially observed, showing that occupants preferred more well-lit environments. In general, window proximity had a larger effect on lighting satisfaction than the measured illuminance parameter.

In terms of acoustics, increased workstation enclosure had positive effects on satisfaction. Private workstations generally had higher variance explained than increases to wall partition height. In a mixed open-plan/private office setting, speech intelligibility and background noise levels both affected satisfaction with acoustics and privacy. Specifically, lower speech intelligibility and background noise both decreased in occupant dissatisfaction. These acoustic parameters had a larger effect size than both the demographic and workstation characteristics in this model. The results suggest that in a typical mixed open-plan/private office setting, 
prioritizing a quiet and well-isolated acoustic environment plays a role in reducing the number of occupants dissatisfied with acoustics and privacy.

The results of satisfaction with ventilation and temperature showed that females were consistently less satisfied than their male counterparts. The differences between the sexes is important and should be considered both in future research of the indoor environment as well as office managers trying to increase occupant satisfaction. Workstation enclosure also had a consistent effect, with occupants in more enclosed workstations reporting higher satisfaction with ventilation and temperature. This result highlights the trade-offs between the various workstation elements; enclosed workstations had positive effects on acoustics satisfaction, but negatively influences satisfaction with acoustics and privacy. The results also partially illustrate the benefits of cooler thermal environments in the offices, with occupants in the open-plan offices reporting higher satisfaction when temperatures and air speeds were lower. In general, the demographic differences and workstation characteristics had larger effect sizes than the measured thermal environment parameters.

In general, these results show that the workstation conditions can affect occupant satisfaction. Designers and planners should be aware of the effects of the various workstation elements, in particularly some of the trade-offs. For instance, window proximity had benefits to lighting satisfaction, but had negative effects on satisfaction with ventilation and temperature. Designers should be cognisant of this, and try to incorporate the benefits of having more windows while addressing some of the negative effects by focussing on improved window thermal performance (R-value, air leakage etc.). 


\subsection{Research Question \#2}

The analyses for the second research question originally set out to evaluate the relationship between various IEQ guidelines limits on occupant satisfaction. The results suggest that adherence to the desktop illuminance recommendations of 300-500 lux by the IESNA RP-112 (2012) standard had some benefits to occupant lighting satisfaction, over workstations with illuminance levels below 300 lux. This observation suggests that satisfying the minimum threshold more important in achieving higher lighting satisfaction than staying strictly within 300-500 lux. However, only a small variance was explained by this parameter, suggesting that meeting these required lighting levels only had a small effect on lighting satisfaction.

The results of the analyses did not show any significant increases in occupant satisfaction when the measured physical parameters were within recommended ranges of the acoustics and thermal comfort criteria (ASA S12.2, ASHRAE 55). Furthermore, the analysis results of the COPE sample indicate that occupants were more satisfied with ventilation and temperature when thermal conditions were cooler than the ASHRAE 55 comfort zone. The explained variance of these parameters were small, indicating that within typical conditioned office spaces, physical IEQ only had a small effect on the occupants. In general, the results did not confirm the field effectiveness of the acoustics and thermal comfort reference criteria in improving occupant satisfaction.

\subsection{Research Question \#3}

The third section of this thesis sought to explore the effects of the broader building amenities outside of the immediate workstation on occupant satisfaction and organizational productivity metrics. The results generally show that after controlling for other variables, satisfaction with building amenities contributed to the occupants' overall satisfaction and 
organizational productivity indices. In particular, satisfaction with amenities related to health and well-being had a persistent effect on the outcome measures. The explained variance of satisfaction with amenities variables were generally comparable to that of workstation environment, particularly in the organizational productivity outcomes. This suggests that amenities do play a role in organizational productivity, and that designers and workplace decision-makers should consider more than only the immediate workstation when looking to improve performance. Specifically, health and well-being amenity features such as access to and quality of drinking water, biophilia, fitness facilities, and public space maintenance should be prioritized in building and office design. This result warrants the exploration of building amenities in future post occupancy building research.

The results also illustrated the relative importance of the components of IEQ, where satisfaction with acoustics and privacy was generally found to more important than lighting and temperature in affecting overall satisfaction and the productivity outcomes. Practitioners and researchers should place a larger focus on this aspect of the indoor environment and ensure that occupants are satisfied with their acoustic environment. The analyses also examined the influence of flexible workstations on occupants where some benefits of this mode of office accommodation were observed. However, this result should be interpreted cautiously due to the nature of the host organization, where flexible workstations were only limited to within certain work groups.

\subsection{Recommendations for Future Research}

In the analysis of the physical workstation parameters, there were several limitations to the methodology that were highlighted in the discussion of the results. Adjustments to the data collection could be implemented in future research to gain more insightful results. For instance, 
perhaps the use of more longitudinal in-place sensors over longer periods could be more indicative of the workstation environment in relation to occupant perceptions. The use of wearable sensors or other methods of gathering additional information on occupant behaviour can also lead to more accurate inputs in terms metabolic rates, clothing values, and personal adjustments to the indoor environment (use of task lighting, shades, headphones etc.).

Conversely, getting additional data using similar data collection methods to build up the number of cases in the database is also meaningful. This will not only aid in the statistical power of the analyses, but it allows for the inclusion of more (and potentially more telling) predictors into the models. For instance, future studies can investigate into the in-situ effects of different building features such as HVAC systems, window specifications (R-value, SHGC etc.), interior finish materials and specific light fixture types on occupants.

Additionally, future works can also explore other avenues of data analysis. There are many different valid statistical procedures and set-ups, each with its benefits and drawbacks. The use of multiple regression in this thesis only evaluates the direct relationships between the predictor and the outcome measures. Future research involving the use of more advanced techniques such as artificial neural networks (ANN) or structural equation modelling (SEM) used in combination with new collected data could provide more insight on the indirect relationships between these parameters.

In the analyses of the broader building amenities, it is important to bear in mind that measures of amenities satisfaction were used as predictors rather than their presence. These satisfaction measures were not necessarily indicative of the influence of specific building amenities physically provided to occupants. Furthermore, occupants were located at the same site and reported their satisfaction on the same provided amenities features. As such, additional work 
is necessary to further investigate the effects of the presence of various building amenities.

Future research into the influences of actual provided amenities elements should be conducted to provide more concrete recommendations on how to better design and optimize building amenities features, and to better guide designers and building operators in their implementation. Future studies investigating the influence of flexible working on occupant satisfaction and productivity is also valuable. 


\title{
APPENDIX A - Church Street Development Project Report
}

\author{
Department of Architectural Science \\ Ryerson University \\ Pre-Occupancy Evaluation Report \\ Daphne Cockwell Health Sciences Complex - Church Street \\ Development Project
}

August 162019 


\section{Executive Summary}

The report details the first phase of a two-phase pre- and post-occupancy evaluation project undertaken for Facilities Management and Development (FMD) at Ryerson University. The project involves evaluating the working spaces of the occupants who will be moving into the new Daphne Cockwell Health Sciences Complex (DCC). Phase 1, reported here, involves evaluating their current workspaces prior to moving into the new building. Phase 2, to be undertaken once the move to the new building is complete, will evaluate the new spaces and compare results. The groups that will be relocating include all faculty and staff from the Ryerson Schools of Midwifery, Occupational and Public Health, Nursing, and Nutrition. The occupants currently reside in the Podium Building, Kerr Hall South, and Sally Horsfall Eaton Centre.

This pre-occupancy report evaluates the conditions of the current workspace of the occupants who will be relocating. The goal of this project is to objectively evaluate the functionality and performance of the current workspaces and to assess indoor environment conditions and its effects on occupant comfort, well-being, and productivity. This information serves as an evaluation of the effectiveness of current spaces. It also acts as a benchmarking tool to assess the effectiveness of the newly designed DCC building after relocation.

A team from the Ryerson University Department of Architectural Science conducted prerelocation collection of data at the currently occupied buildings in May 2018. The data collected consisted of measurements of the physical environment (e.g. thermal, acoustic, air quality, lighting) at representative locations, and a questionnaire administered to all occupants about multiple facets of environmental satisfaction, workplace productivity, and aspects of workplace functionality, safety, and accessibility.

Questionnaire responses were received from 25 occupants across all departments, and spot physical measurements were collected at 42 workspaces across the buildings. A summary of the results from the occupant surveys are shown below in Figure 1. 


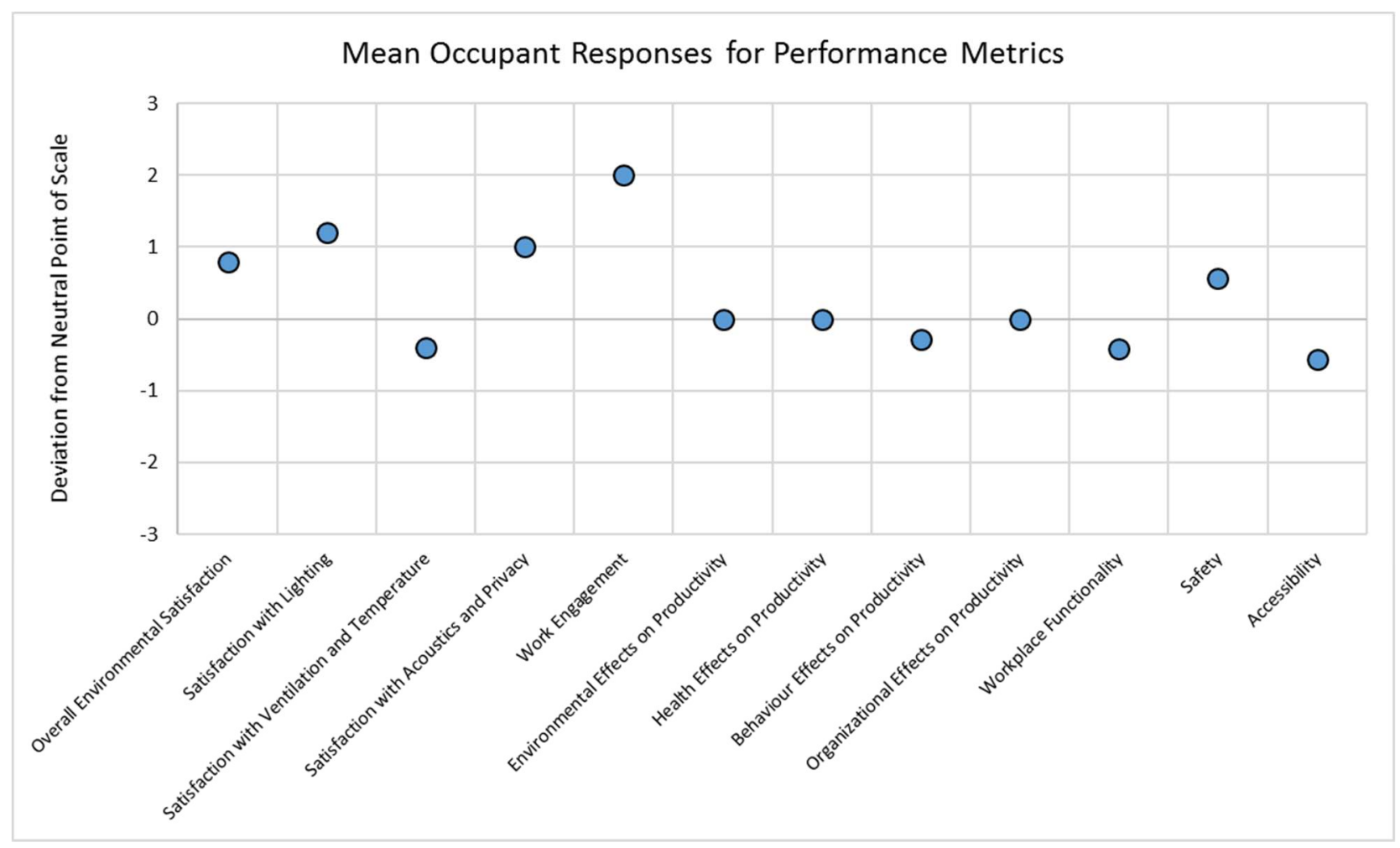

Figure 1. Summary of Occupant Survey Responses.

Satisfaction with ventilation and temperature was rated slightly on the lower end of the spectrum, below neutral. This corroborated with the responses in terms of complaints, where $32 \%$ of respondents have complained about the thermal conditions or air quality this season. Thermal sensations in the summer time were extreme, with respondents either being too hot or too cold, indicating that there is insufficient individual temperature control. Physical measurements of the temperature and relative humidity $(\mathrm{RH})$ at the workspaces were mostly within recommended levels, with RH being on the lower side. This could cause discomfort due to dryness for some occupants. The measured indoor $\mathrm{CO}_{2}$ and particulate concentrations were well within the recommended levels.

Satisfaction with acoustics and privacy was generally rated favourably by the respondents, as were the more detailed responses regarding speech sounds and privacy (ability to have a conversation). In general, the measured ambient sound levels at the workstations were within recommendations. In fact, the workstations may have been too quiet in terms of sound masking. This may have negative consequences on the perception of sounds during non-ambient conditions (e.g. foot traffic, meetings, street-level sounds).

Satisfaction with lighting was generally rated favourably by the respondents. In terms of physical measurements of desktop illuminance, most locations were within recommended levels. Only around $26 \%$ of measured locations had lighting levels below recommended values. Considerations could be made regarding increasing the amount of lighting, along 
with adjustable lighting controls and/or shading devices for occupants who prefer dim environments.

With regards to environmental effect on productivity, only approximately $20 \%$ of respondents felt that the indoor environment had an extreme effect on productivity (positive or negative). An overall environmental factor score (out of 100) was calculated to be 46.67. A score of 50 represents a neutral score, where environmental factors have no effect (positive or negative) on productivity. The results indicate that improvements can be made regarding the indoor environment to improve productivity and performance.

An overall health factor score (out of 100) was determined to be 49.21, giving indication of the slight negative effects of health factors on productivity. Adjustments to occupant health (both mental and physical) can have improvements to productivity.

In terms of behavioural effects on productivity (e.g. team morale, interactions and relationships with coworkers), the average response for this variable was slightly below the neutral point. However, like other productivity metrics, only $10 \%$ of respondents felt that behavioural aspects effected their productivity in an 'extreme' manner (score of either 1 or 5). An overall behaviour factor score (out of 100) was calculated to be 28.57, illustrating the negative impacts of behavioural factors on productivity. As such, this is an area of weakness, and improvements to the interaction between colleagues can have benefits to employee productivity.

The average organizational effect on productivity was rated to be at the neutral point of the scale. Like the other productivity metrics only a small fraction of the respondents felt organizational factors had an 'extreme' effect on productivity. An overall organizational effect score (out of 100) was calculated to be 45.24, showing that organizational factors have a negative impact on productivity. Improvements can be made in this area to boost productivity.

Work engagement was highly rated overall, indicating that occupants are very engaged in their work overall. The results show that this is an area of strength in the respondents.

The results for both self-assessed quality and volume of work (with respect to organizational expectations) were favourable.

Workplace functionality was viewed somewhat negatively by the occupants, with the average response below the neutral point of the scale. Furthermore, there were no occupants that felt 'very satisfied' in terms of their workplace functionality, suggesting that this is an area that should be improved upon.

In general, workplace safety was viewed positively by the occupants. However

approximately a quarter of the respondents felt their environment was unsafe. Though not an overwhelming number of occupants felt this way, safety is a priority and the sources of these unsafe feelings should be assessed and addressed.

Accessibility was also viewed somewhat negatively by the occupants. Although the majority of responses felt that accessibility was 'typical', only $5 \%$ of responses were 
positive. A review of the open-ended responses suggested that the causes of these poor results were varied, including issues such as elevators, accessibility on weekends, signage, and traveling between buildings on campus. The sources of these complaints were mixed, with some directed at specific buildings while others were regarding the general Ryerson University campus.

It is important to keep in mind the context in which these findings were measured when interpreting the results. The reported physical measurements were based on spot measurements take in May and over a short time (approximately 10-15 minutes) of the workspace. While a large number of locations were sampled, there is no guarantee that these data are representative of the conditions of all offices within these buildings, or at all times and at other seasons. It is likely that measurements made in a different season may have produced different results on many metrics.

Similar considerations apply to survey data. The time of year and how that interacts with the prevailing work environment, along with prior knowledge of the future relocation may all affect the survey outcomes measured. 


\section{Introduction}

The report details the first phase of a two-phase pre- and post-occupancy evaluation project as part of the Facilities Management and Development (FMD) capital project process. The pre-occupancy project establishes the current working conditions of the occupants who will be relocating from their current locations into the new Daphne Cockwell Health Sciences Complex (DCC). The groups that will be relocating include all faculty and staff from the Ryerson Schools of Midwifery, Occupational and Public Health, Nursing, and Nutrition. The data presented in this report is collected from the current office locations of the groups above; a brief description of these workspaces is presented in Section 2.4.

The goal of this project is to objectively evaluate the functionality and performance of the current spaces used by these departments, and to assess indoor environment conditions and its effects on occupant comfort, well-being, and productivity. These evaluations provide a baseline for comparison to the snew DCC building once it is occupied. This will also form the basis for identifying areas of improvement.

The intention is to conduct similar measurements of the DCC following the relocation and population of the building and a settling in period. At the time of writing, the move in is anticipated to be in the Winter of 2019, and so the evaluation should be done about 6 months to a year after. This data will be useful in the interpretation of the pre-post analysis and evaluation of the effectiveness of the various spaces.

This project also serves as a pilot project for the development of a standardized evaluation methodology for similar studies at Ryerson University in the future. This will enable the collection of a set of unique, easily interpretable results localized to the Ryerson setting, and allow for better informed planning decisions in the future.

\section{Methods and Procedures}

The pre- and post-occupancy building evaluations take a multi-dimensional approach to evaluating productivity and the indoor environment conditions. The various elements of this approach are described below. This process was approved by Ryerson University's Research Ethics Board (REB).

\subsection{Occupant Survey}

All occupants at the study site were invited by the Researchers to complete an online questionnaire hosted on SurveyMonkey. The responses were anonymous and do not allow the identification of individual respondents. The occupant survey questions were based on existing survey questions developed by the National Research Council (NRC)'s postoccupancy questionnaire (Leder et al., 2016) and a workplace productivity survey developed by Professor Jennifer McArthur (Carey, Bortoluzzi, \& McArthur, in press). Survey items were chosen to address elements relevant to indoor environment conditions and workplace productivity, functionality, safety, and accessibility. 
The questionnaire was organized into 7 modules; Table 1 provides a brief description of each module. All occupants were asked to complete the first 4 core modules, with the option to complete the additional modules regarding noise, thermal comfort, and demographics. This approach was taken to keep the time burden more reasonable for respondents. Respondents were also invited to provide open ended comments at various points in the survey to provide an opportunity to highlight issues that weren't covered by the survey, as well as to give the researchers some context and qualitative information.

The occupant survey was distributed to all faculty and staff in the Schools of Midwifery, Occupational and Public Health, Nursing, and Nutrition. The questionnaire was completed by a total of 25 occupants; a relatively small sample out of the total population of approximately 120 faculty and staff. Therefore, a separate analysis could not be performed for each individual department, and the results presented herein are the aggregate of the responses from every department.

Table 1 - Summary description of questionnaire modules

\section{Module Description}

Core Module \#1 Office location; department; workstation information

Core Module \#2 Environmental satisfaction (ventilation \& temperature, lighting, acoustics \& privacy, overall environment)

Core Module \#3 Environmental, health, behaviour, and organizational effects on productivity; work engagement

Core Module \#4 Workplace functionality (local amenities); safety; accessibility

Optional Module Optional questions on workplace acoustics, noise, and privacy \#1

Optional Module Optional questions on thermal comfort \#2

Optional Module Optional questions on demographics (gender, age, ethnic \#3 background)

\subsection{Physical Measurements of Indoor Environment}

Physical measurements of the occupied spaces were made using various sensors mounted on a platform and supplemented with handheld sensors where applicable. Figure 2 shows an image of the platform used. The measurements were taken at the workstation of the occupant of the space, in particular where they would typically be seated. This is consistent 
with other studies of this type (Choi, Loftness, \& Aziz, 2012; Newsham, Veitch, \& Charles, 2008; Newsham et al., 2013).

Measurements were made at representative locations on all floors occupied by typical faculty and staff. It is important to note that measurement locations were chosen to be representative, but measurements were not taken at all possible locations. Data was collected from a total of 42 workspaces across all departments.

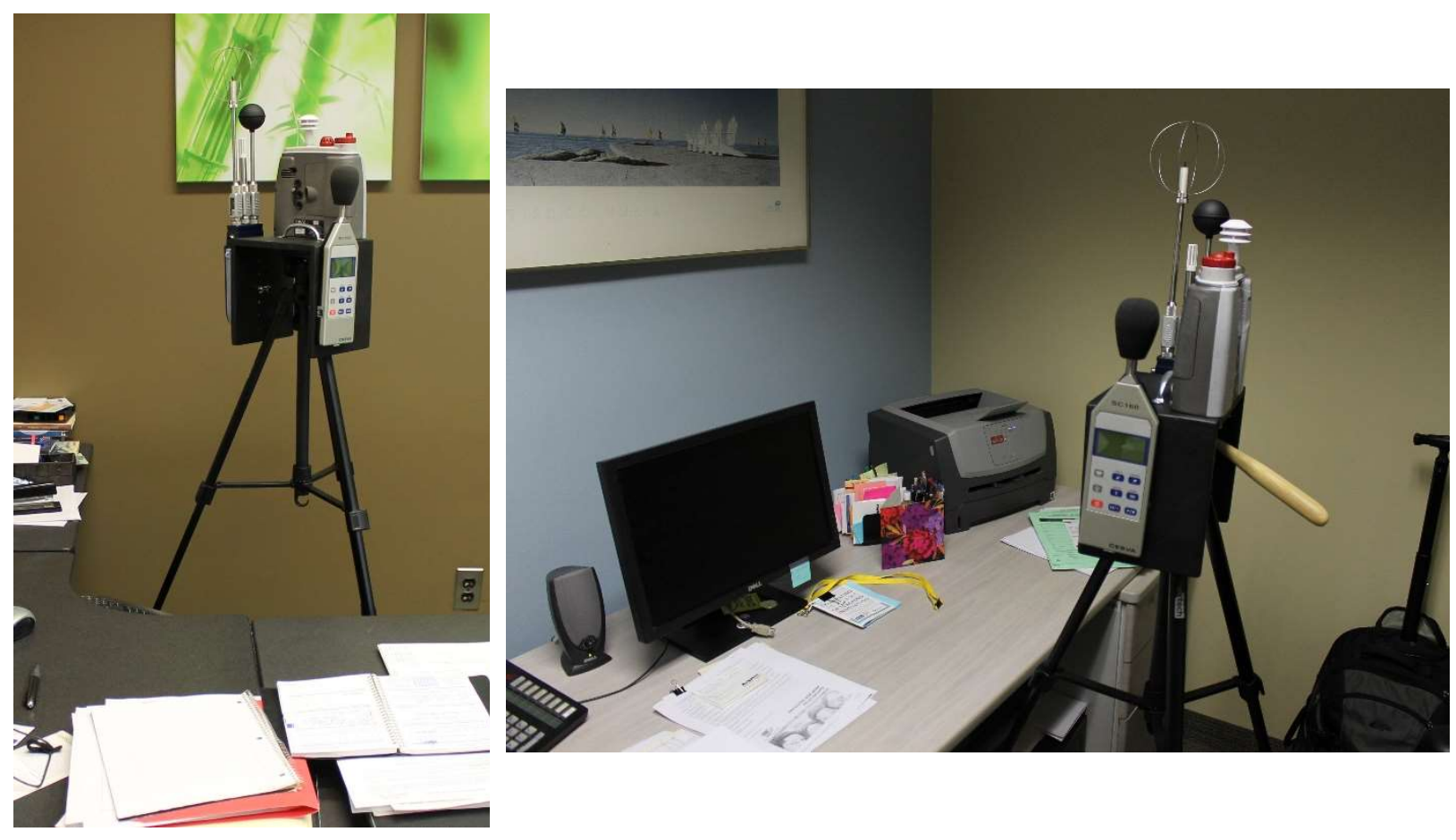

Figure 2. Sensor platform used for physical indoor environment measurements.

The physical measurements taken are detailed snapshots of indoor environment conditions over a 10-15 minute period at representative locations within the buildings. Table 2 summarizes the instrumentations/sensors used in this study. ASHRAE Standard 55 (2017) specifies that the measurement positions above ground appropriate for the determination of thermal comfort for seated occupants are $0.1 \mathrm{~m}$ (ankle), $0.6 \mathrm{~m}$ (torso), and $1.1 \mathrm{~m}$ (head) for air temperature and air speed. Due to instrumentation constraints, this study contains only measurements taken at the head level, which is often the only reported metric in published studies of this type.

Data collection from the instruments/sensors was semi-automated using built-in sensor timers where applicable. Measurements such as desktop illuminance and sound levels were manually controlled by the researcher. During this process, the researcher also manually recorded several other workstation characteristics:

- Windows and shading devices

- Office area 
- Diffuser and exhausts

- Task lighting

- Printers and other appliances

- Interior finishes

Table 2 - Summary information on sensor platform instruments/sensors

$\begin{array}{llll}\text { Instrument/Sensor } & \text { Parameter Measured } & \text { Range } & \text { Accuracy } \\ \text { 3M EVM-7 } & \text { Carbon Dioxide } & 0 \text { to } 5000 \mathrm{ppm} & \pm 100 \mathrm{ppm} \\ & \text { Particulates } & 0 \text { to } 200 \mathrm{mg} / \mathrm{m}^{3} & \pm 15 \% \\ & \text { Air Temperature } & 0^{\circ} \mathrm{C} \text { to }+60^{\circ} \mathrm{C} & \pm 1.1^{\circ} \mathrm{C} \\ & \text { Relative Humidity } & 0 \text { to } 100 \% \mathrm{RH} & \pm 5 \% \mathrm{RH} \\ \text { Delta Ohm HD } 32.3 & \text { Radiant Temperature } & -10^{\circ} \mathrm{C} \text { to }+100^{\circ} \mathrm{C} & \pm 0.19^{\circ} \mathrm{C} \\ & \text { Air Speed } & 0 \text { to } 5 \mathrm{~m} / \mathrm{s} & \pm 0.2 \mathrm{~m} / \mathrm{s} \\ & \text { Air Temperature } & -40^{\circ} \mathrm{C} \text { to }+100^{\circ} \mathrm{C} & \pm 0.19^{\circ} \mathrm{C} \\ \text { CESVA SC160 } & \text { Relative Humidity } & 0 \text { to } 100 \% \mathrm{RH} & \pm 1.5 \% \mathrm{RH} \\ \text { Extech HD450 } & \text { Sound Level } & 0 \text { to } 137 \mathrm{~dB} & \pm 5 \mathrm{~dB} \\ & \text { Illuminance } & 0 \text { to } 400,000 \text { lux } & \pm 5 \%\end{array}$

Practical considerations prevented data collection at all possible occupant locations in the buildings (e.g. laboratories, lecture rooms etc.). The focus of these measurements was on office spaces, as the single space type where the occupants spend most of their time. To minimize disruption to building occupants, workstations that were temporarily unoccupied (e.g. usual occupant at meeting, out of office, on vacation) were prioritized as measurement locations. It is assumed that these measurements are representative as they are all served by common building systems. The physical measurements were made during normal working hours only.

Further, only spot measurements were taken of the physical workstations and only in the month of May. As such the results are limited to the conditions of the indoor environment during a relatively short period of time. Thus, just because a measurement at a specific location may have violated (or met) a standard during the visit does not mean that these conditions necessarily persisted outside of the study period. However, they indicate that further investigation may be warranted. If specific identified issues are to be pursued, a 
more comprehensive supplementary set of measurements should be gathered. Nonetheless, the results provide valuable insights regarding the function and conditions of the office spaces.

\subsection{Measurement Dates}

On-site visits and physical data collection were conducted between May $14^{\text {th }}$ and May $23^{\text {rd }}$ of 2018. An initial invitation to participate in the online questionnaire was sent to occupants of all four departments on April 24th 2018, with reminders sent out on May $8^{\text {th }}$, 2018. The survey was closed to responses on May 13 ${ }^{\text {th }}, 2018$.

Figure 3 shows the outdoor weather conditions during period of site visit, measured at Billy Bishop Toronto City Airport.

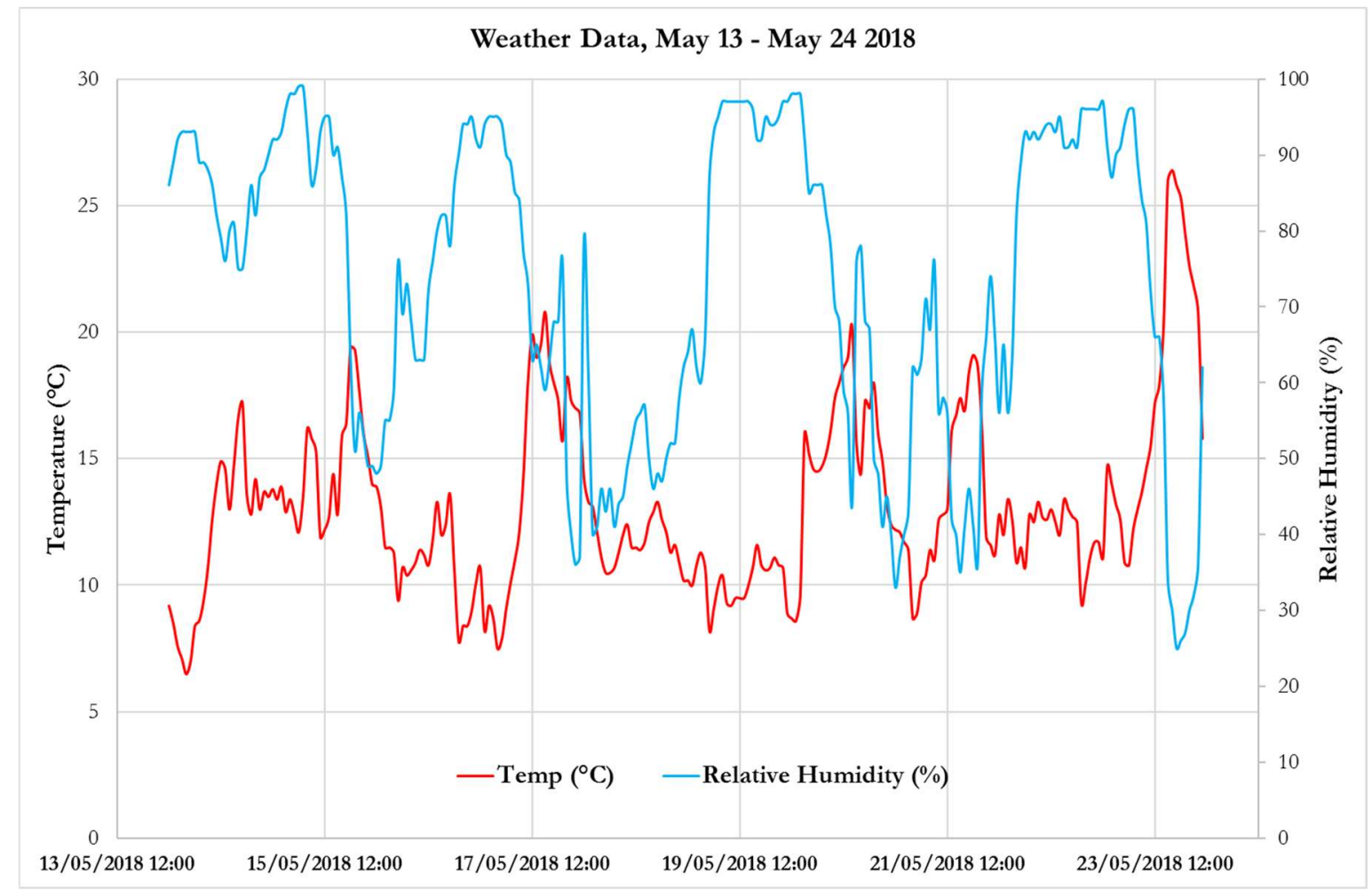

Figure 3. Weather conditions during site visit, taken at Billy Bishop Toronto City Airport.

\subsection{Building Description}

Table 3 provides basic descriptive information on the current office locations of the various departments involved in this study. Figures 4-7 show photographs of example workstations of the respective departments. 
Table 3 - Current office locations of occupants

\section{Department Building Description}

Nursing

Occupational

\& Public

Health

Midwifery

Nutrition

\section{Sally Horsfall}

Eaton Centre

Constructed in 1971, the Podium building is a concrete structure with a brick masonry façade. The windows in this building are single glazed and some are operable. The offices are typically equipped with internal shading device (roller shades or horizontal shutters) and have suspended ceilings. The primary source of heating is provided using steam through a steam to water exchanger which then uses the hot water to heat the building. The cooling system is a centralized system using chilled water from the chilled water plant on the Ryerson Campus.

Sally Horsfall Eaton Centre was constructed in 2002 as an expansion above the existing Eric Palin Hall and share many of the same infrastructure. SHE is a concrete structure with an aluminum composite panel cladding system. The windows in this building are double-paned insulated glazing units (IGUs). The offices are typically equipped with internal shading devices (roller shades) and suspended ceilings. The HVAC in the building consists of a centralized supply provided by AHUs at the roof level. Steam is the primary source of heating in the building. Cooling is provided by the chilled water from the chilled water plant on the Ryerson Campus.

Constructed in around 1960, Kerr Hall South is a concrete structure with a solid masonry façade. The windows in this building are single glazed and operable. The offices are typically equipped with internal shading devices (roller shades) and stucco ceilings (popcorn ceilings). The primary source of heating is provided by baseboard heaters using steam. The cooling system is a centralized system using chilled water from the chilled water plant on the Ryerson Campus. 


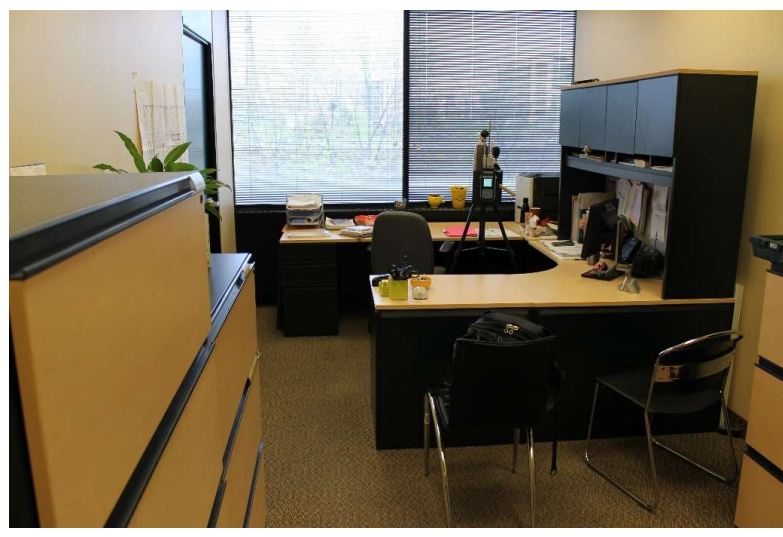

Figure 4. Example School of Nursing Workstation.

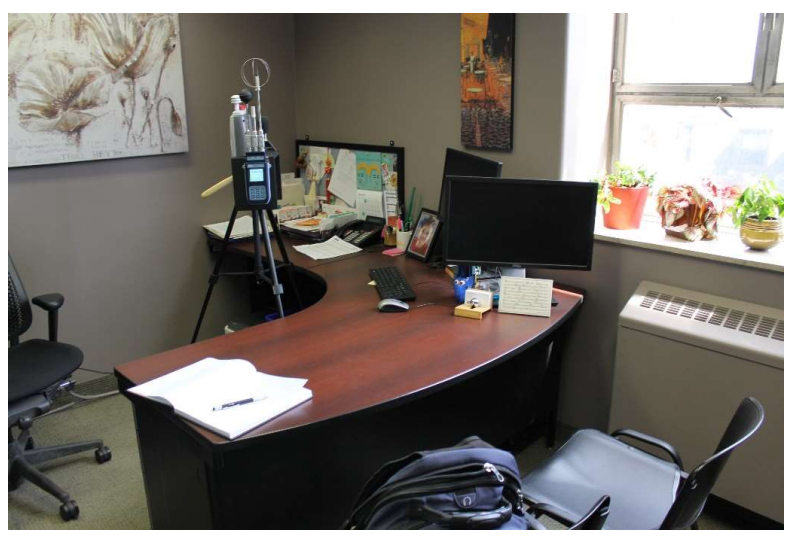

Figure 6. Example School of Nutrition Workstation.

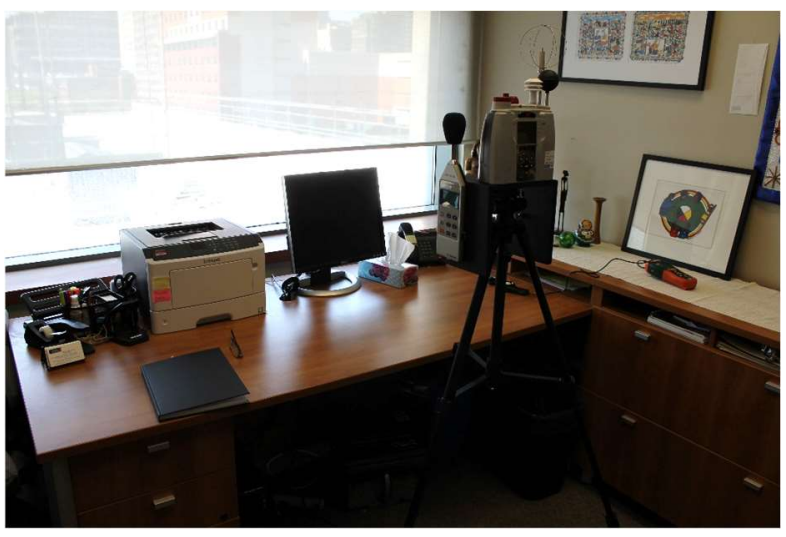

Figure 5. Example Midwifery Education Workstation

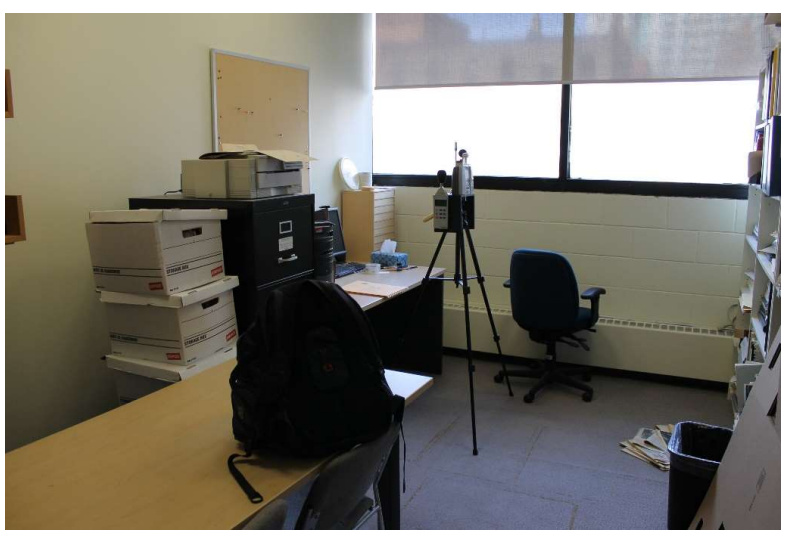

Figure 7. Example School of Occupational \& Public Health Workstation.

\section{Results and Discussions}

The results presented are descriptive and are limited to a subset of the data judged most relevant to facilities management and the individual departments involved. Where applicable the descriptive data was compared from the study buildings to standards, recommended practice, benchmarks, or findings from other research projects with similar contexts and outcomes. No detailed statistical analyses are presented in this report.

\subsection{Demographics}

Information on demographics and time spent at the workstation are used primarily to provide context for the results of this report. Table 4 shows the demographic information of the respondents to the survey questionnaire and Table 5 shows the respondents' average time spent at their workstations in a typical week. The respondents to this survey were predominantly women and the majority in the 45 to 65 age range. This can affect the 
outcomes on certain questionnaire items, as previous research has demonstrated differences in environment perception and satisfaction between the genders (Brookes \& Kaplan, 1972; Choi et al., 2012; Leder et al., 2016; Newsham et al., 2008; Yildirim, AkalinBaskaya, \& Celebi, 2007). Other than gender, the demographic factors were fairly evenly distributed. Respondents typically spends a significant portion of their time at their workstations, allowing for a representative evaluation of opinions on the space.

Table 4 - Demographics information

\begin{tabular}{|c|c|c|c|c|c|}
\hline & $\%$ & $\%$ & $\%$ & $\%$ & $\%$ \\
\hline Department & $\begin{array}{l}\text { Midwifery } \\
20\end{array}$ & $\begin{array}{l}\text { Nursing } \\
20\end{array}$ & $\begin{array}{l}\text { Nutrition } \\
36\end{array}$ & $\begin{array}{l}\text { Occupational and Public Health } \\
\qquad 24\end{array}$ & \\
\hline $\begin{array}{l}\text { Window to Exterior in } \\
\text { Workstation }\end{array}$ & $\begin{array}{c}\text { No Window } \\
40 \\
\end{array}$ & $\begin{array}{l}\text { Yes Window } \\
60 \\
\end{array}$ & & & \\
\hline Biological Sex & $\begin{array}{c}\text { Female } \\
94 \\
\end{array}$ & $\begin{array}{c}\text { Male } \\
6\end{array}$ & $\begin{array}{c}\text { Transitioned/In-transition } \\
0\end{array}$ & $\begin{array}{c}\text { Prefer not to disclose } \\
0\end{array}$ & \\
\hline Age Range & $\begin{array}{c}25 \text { and under } \\
0\end{array}$ & $\begin{array}{c}26-44 \\
27\end{array}$ & $\begin{array}{c}45-65 \\
60\end{array}$ & $\begin{array}{c}66 \text { and above } \\
0\end{array}$ & $\begin{array}{c}\text { Prefer not to disclose } \\
13\end{array}$ \\
\hline
\end{tabular}

Table 5 - Time spent at workstation in a typical week, self assessed

\begin{tabular}{|c|c|}
\hline $\begin{array}{c}\text { Hours Spent at Workstation } \\
\text { in Typical Week }\end{array}$ & $\%$ \\
\hline$<\mathbf{8}$ & 4 \\
$\mathbf{8 . 0}-\mathbf{1 5 . 9}$ & 12 \\
$\mathbf{1 6 . 0}-\mathbf{2 3 . 9}$ & 8 \\
$\mathbf{2 4 . 0}-\mathbf{3 1 . 9}$ & 40 \\
$>\mathbf{3 2 . 0}$ & 36 \\
\hline
\end{tabular}

\subsection{Indoor Environment Quality and Satisfaction}

\subsubsection{Thermal Comfort and Air Quality}

Figures 8 below shows the aggregate survey results from Core Module \#2 regarding satisfaction with ventilation and temperature. This metric is the combined effect of both air quality and thermal comfort, as ventilation influences not only indoor pollutant concentrations but also indoor temperatures and thermal perceptions. Specific results regarding thermal comfort and air quality will be discussed separately in more detail in Sections 3.2.1.1 and 3.2.1.2.

A wide spectrum of responses was reported regarding satisfaction with ventilation and thermal comfort. The mean score of satisfaction with ventilation and temperature was 3.6, below the neutral point of the scale. Overall, this suggests this dimension of satisfaction is a potential area of concern; $32 \%$ of respondents indicated dissatisfaction with conditions (rating < 3). This result is consistent with Table 6, which shows that approximately onethird of the respondents had complained to a facilities manager or supervisor regarding the thermal and/or air quality conditions at their workstation. 


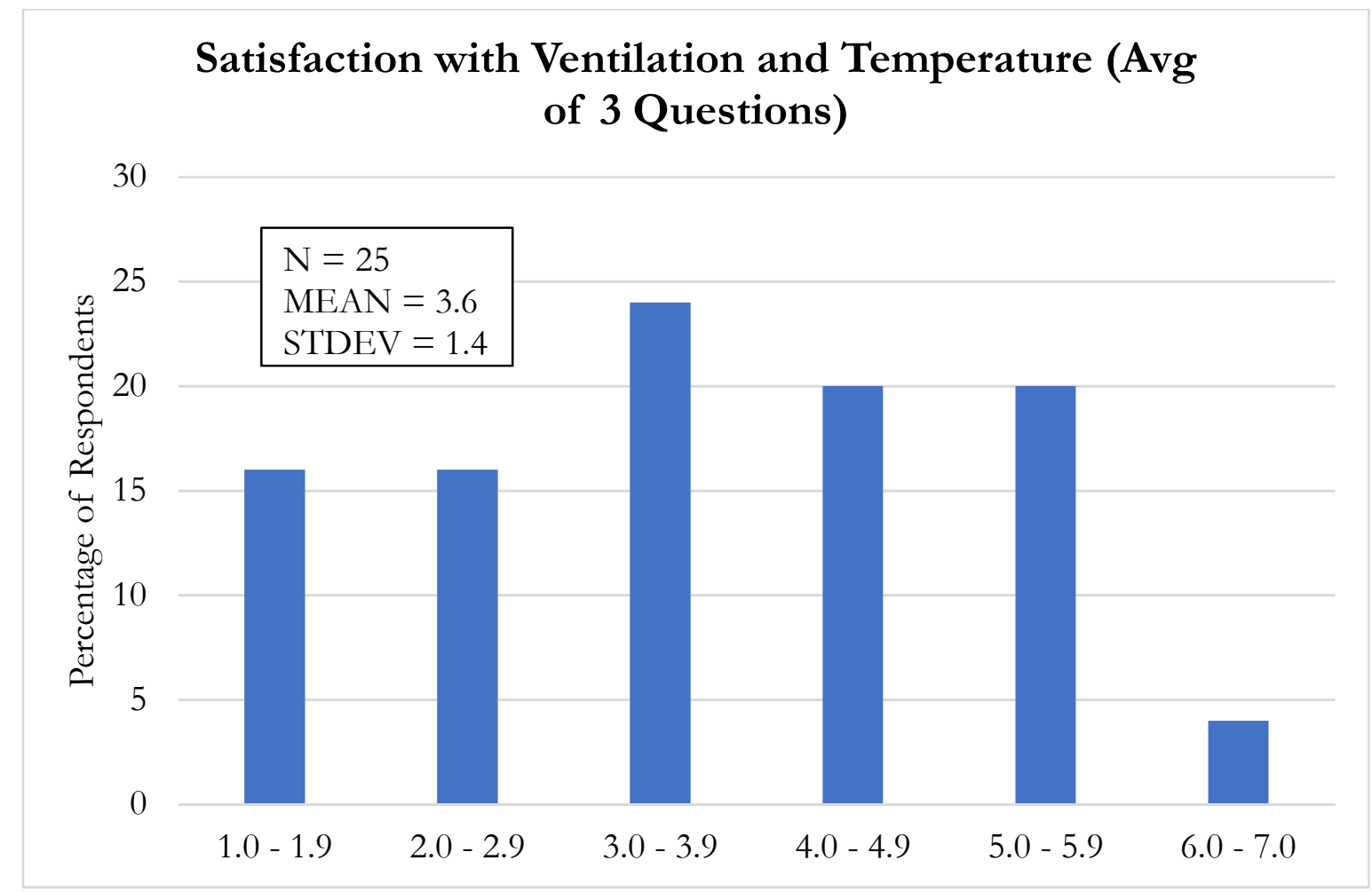

Figure 8. Satisfaction with ventilation and temperature; $1=$ =ery unsatisfactory, $4=$ neutral, $7=$ very satisfactory.

Table 6 - Complaints to facility manager regarding thermal conditions or air quality.

\begin{tabular}{|c|c|}
\hline $\begin{array}{c}\text { Have you complained to a facility manager or } \\
\text { supervisor this season about the thermal conditions } \\
\text { or air quality in your work space? }\end{array}$ & $\begin{array}{c}\% \text { of } \\
\text { Respondents }\end{array}$ \\
\hline No Response & 28.0 \\
No & 40.0 \\
Yes & 32.0 \\
\hline
\end{tabular}

\subsubsection{Thermal Comfort}

Figures 9-11 below show ratings for select questions in the optional thermal comfort module of the survey. ASHRAE Standard 55 (2017) suggests that a neutral sensation is the optimal desired state. On average, thermal sensations were neutral and evenly distributed in the winter months. Although the average result was neutral in the summer months, this was a result of respondents being either too cold or too hot. This is indication of insufficient temperature regulations and lack of individual thermal controls across the occupants.

Situations where the occupants were too cold, resulting in the taping the supply vents (due to lack of ability to shut off A/C) was observed at several locations (refer to Figure 12). 


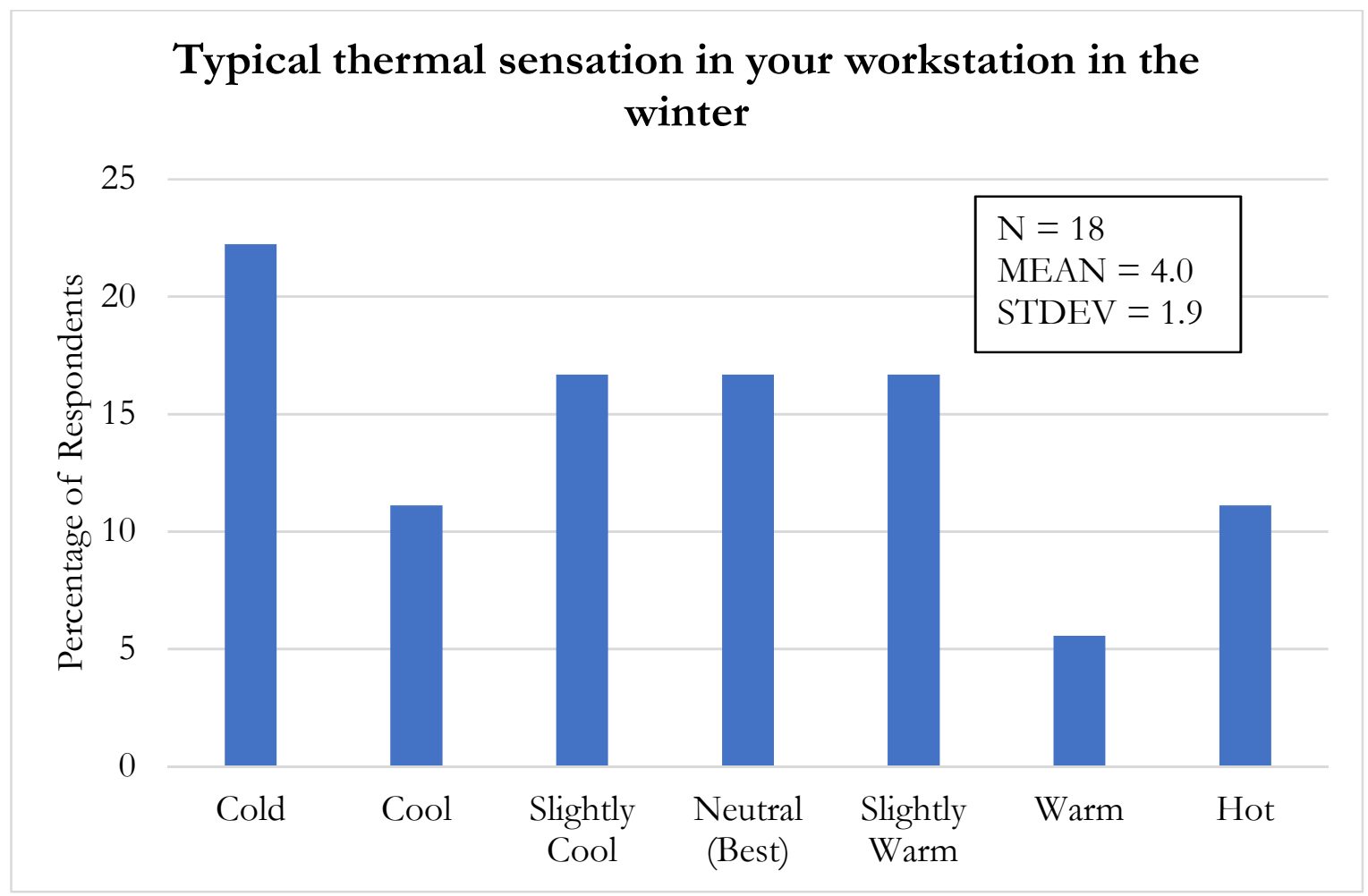

Figure 9. Typical thermal sensation in winter; $1=$ cold, $4=$ neutral, $7=$ hot.

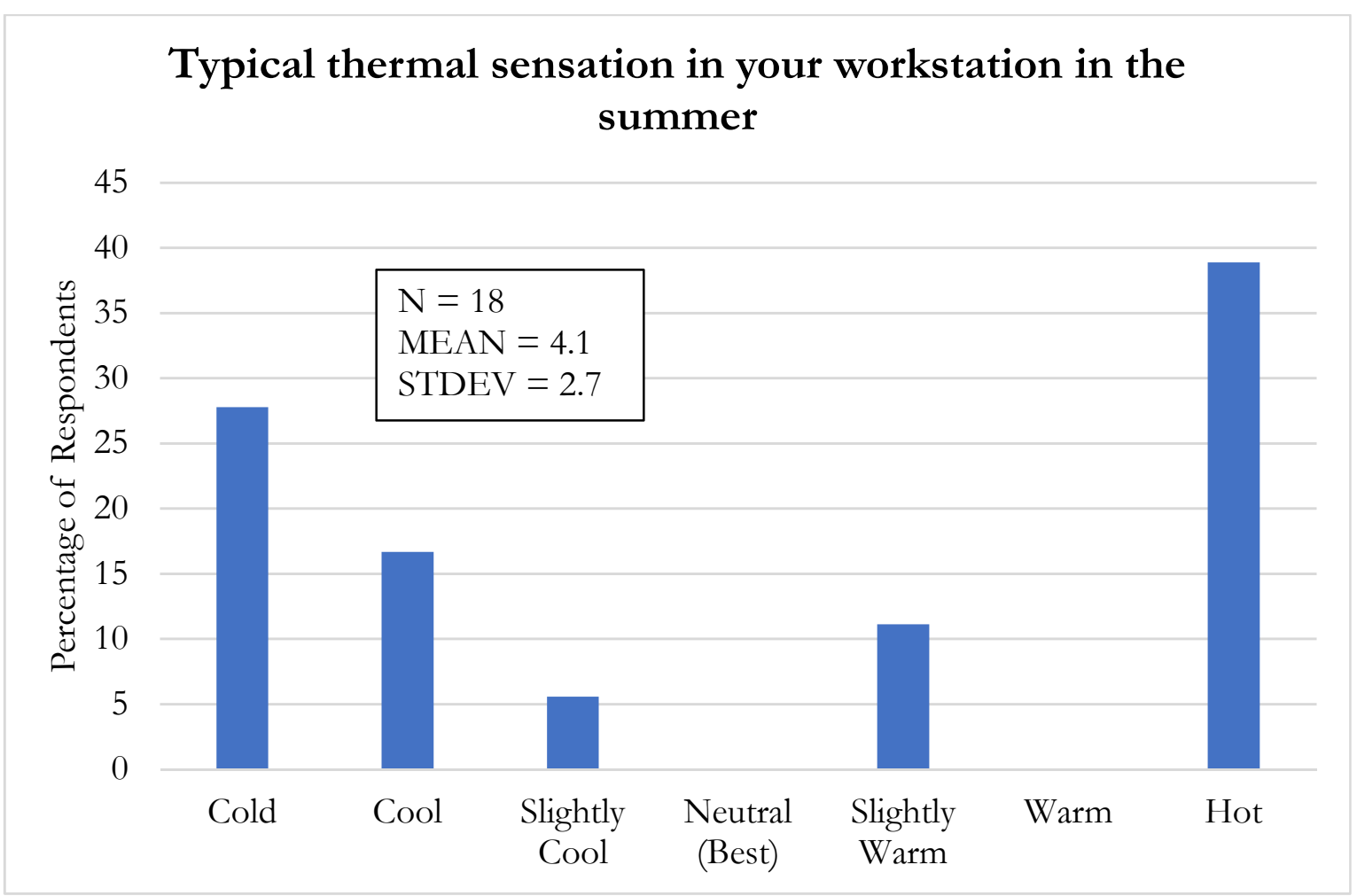

Figure 10. Typical thermal sensation in summer; $1=$ cold, $4=$ neutral, $7=$ hot. 


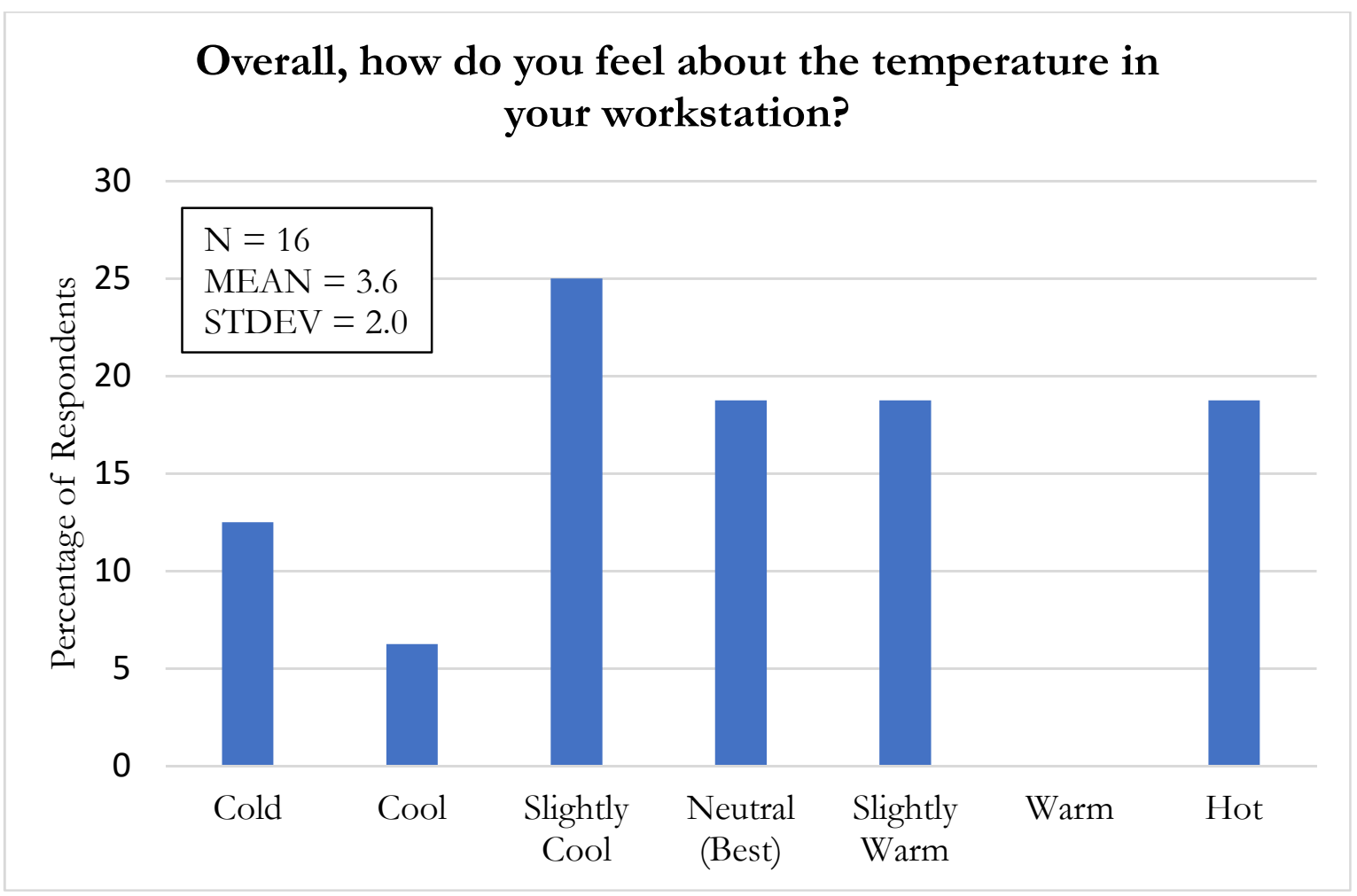

Figure 11. Overall thermal perception in workstation; $1=$ cold, $4=$ neutral, $7=$ hot.

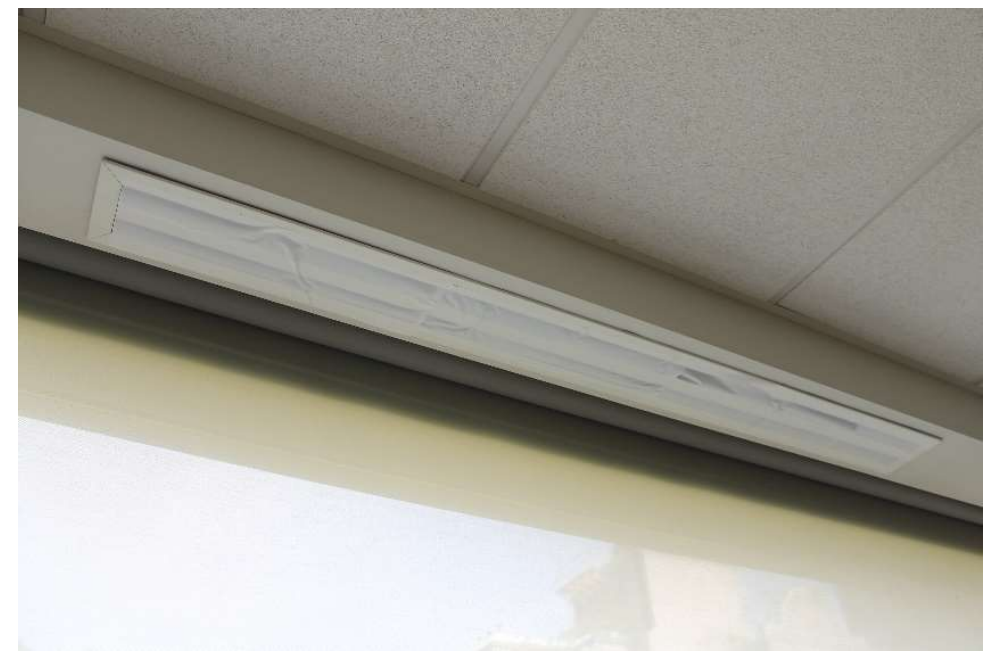

Figure 12. Occupants taped supply vent shut due to cold air.

Figure 13-15 shows physical data related to thermal comfort. In general, air temperatures were consistent across all the measurement locations, with a mean air temperature of $23.9^{\circ} \mathrm{C}$ and a standard deviation of $1.0^{\circ} \mathrm{C}$. Relative humidity (RH) at the measured workstations had a mean of $45.2 \%$ and a standard deviation of $10.5 \%$. Recorded air speeds were typically very low with a mean air speed of $0.045 \mathrm{~m} / \mathrm{s}$ (standard deviation of 0.064 $\mathrm{m} / \mathrm{s}$ ), where more than $97 \%$ of the measured locations had an average air speed of less than $0.2 \mathrm{~m} / \mathrm{s}$. 
The ASHRAE 55 standard is the most commonly referenced standard related to thermal comfort in North American office spaces. This standard recommends that the interior air temperature for typical office workers be maintained in the range of approximately 21 $26^{\circ} \mathrm{C}$. In terms of RH there is no lower limit, but in literature a minimum RH level of approximately 25 - 30\% is recommended to avoid static electricity shocks, drying of the mucus membranes, and break-up of tear film in the eyes (McIntyre, 1978). An upper limit of approximately $70 \% \mathrm{RH}$ is recommended in the ASHRAE 55 standard. With regards to air speed, the standard suggests that there is little risk of local draughts below approximately $0.15 \mathrm{~m} / \mathrm{s}$.

Measured temperatures in the buildings were typically within ASHRAE recommendations, with the exception of one measured location which was at $26.4^{\circ} \mathrm{C}$. Humidity in the building was generally at the lower end of the desired range, with approximately $22 \%$ of the measured locations at the lower ranges of the recommended level. Air speed was generally low at the measured workstations, except one location where the measured air speed was high enough to cause a draught risk.

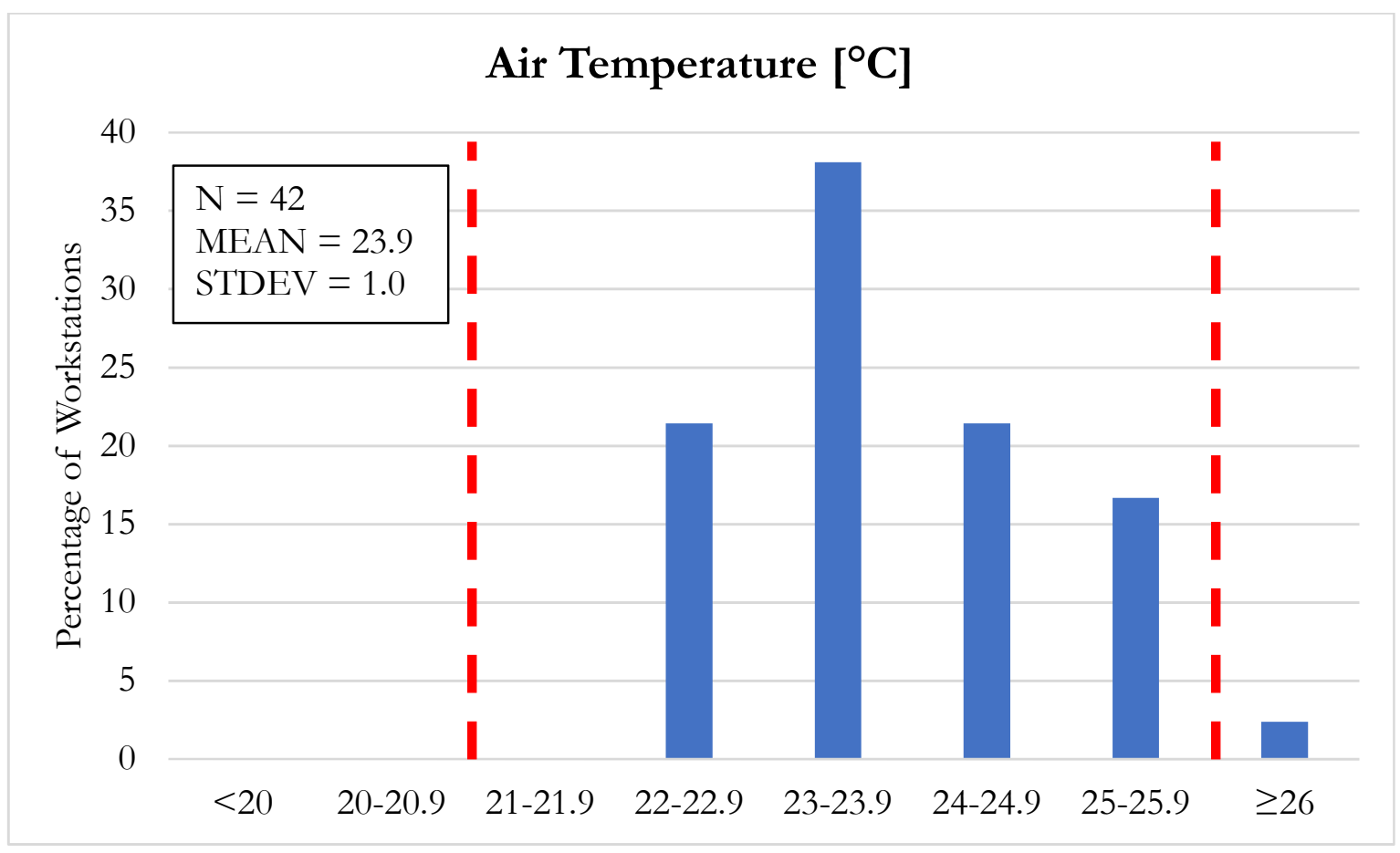

Figure 13. Air temperature in workstation at head level. 


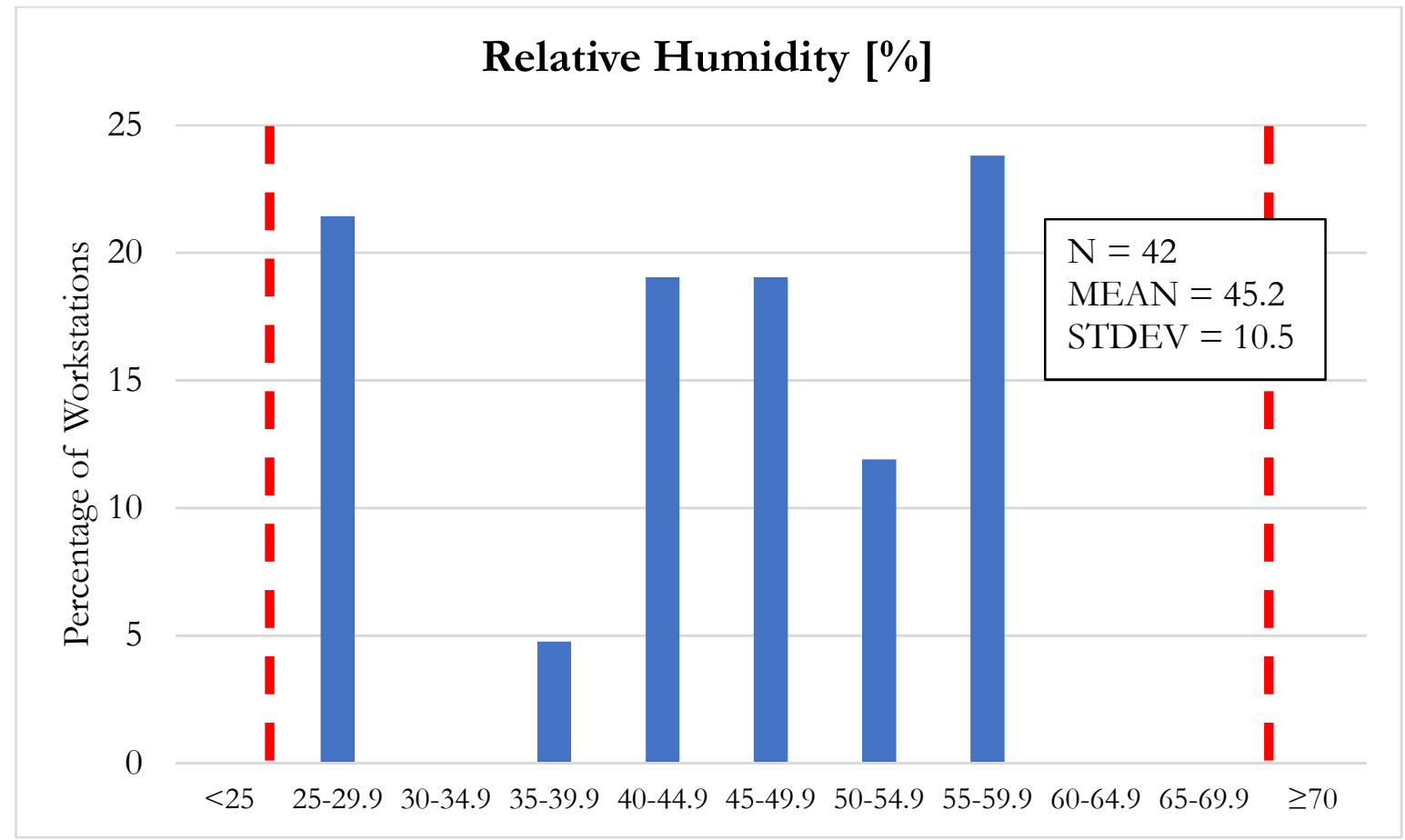

Figure 14. Relative humidity in workstation at head level.

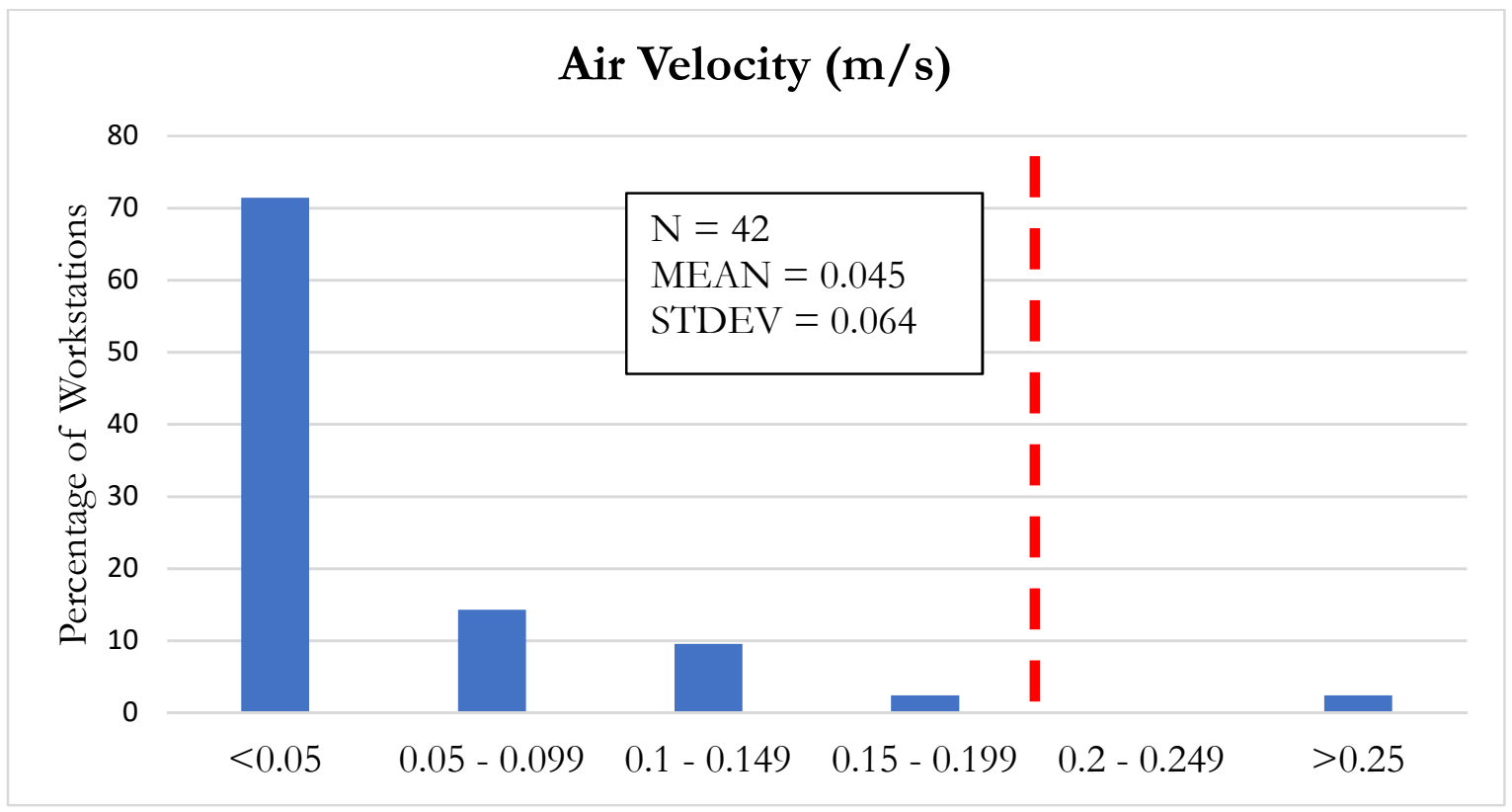

Figure 15. Air speed in workstation at head level.

\subsubsection{Air Quality}

Figure 16 shows $\mathrm{CO}_{2}$ concentrations measured at the workstations. Typical $\mathrm{CO}_{2}$ concentrations outdoors are around $375 \mathrm{ppm}$, with elevated values indoors primarily due to human respiration. Although not harmful in itself at typical indoor concentration levels, 
high concentrations may be an indication of poor ventilation effectiveness, with potentially negative consequences for the dilution of other more harmful pollutants.

ASHRAE Standard 62.1 (2016) ventilation rates are based on indoor $\mathrm{CO}_{2}$ concentrations below 700 ppm above ambient outdoor air (i.e. 1075 ppm). (Seppanen, Fisk, \& Mendell, 1999) noted several studies suggesting decreases in sick building syndrome (SBS) symptoms with concentrations below 800 ppm. (Apte, Fisk, \& Daisey, 2000) found significantly increased odds for certain SBS symptoms with $\mathrm{CO}_{2}$ concentrations $250 \mathrm{ppm}$ above outdoor levels ( $\sim 625 \mathrm{ppm})$.

The $\mathrm{CO}_{2}$ levels at the measured workstations were well below ASHRAE recommended values used to determine ventilation rates, with an average value of $564 \mathrm{ppm}$. Approximately $90 \%$ of the locations were below the most stringent recommendation of $625 \mathrm{ppm}$. However, it should be noted that many occupants at the measured workstations have not been regularly occupying their offices, as the measurements took place during summer and post-exam season. The $\mathrm{CO}_{2}$ concentrations are potentially skewed to the lower end of the spectrum as a result of the reduced occupancy and human activity.

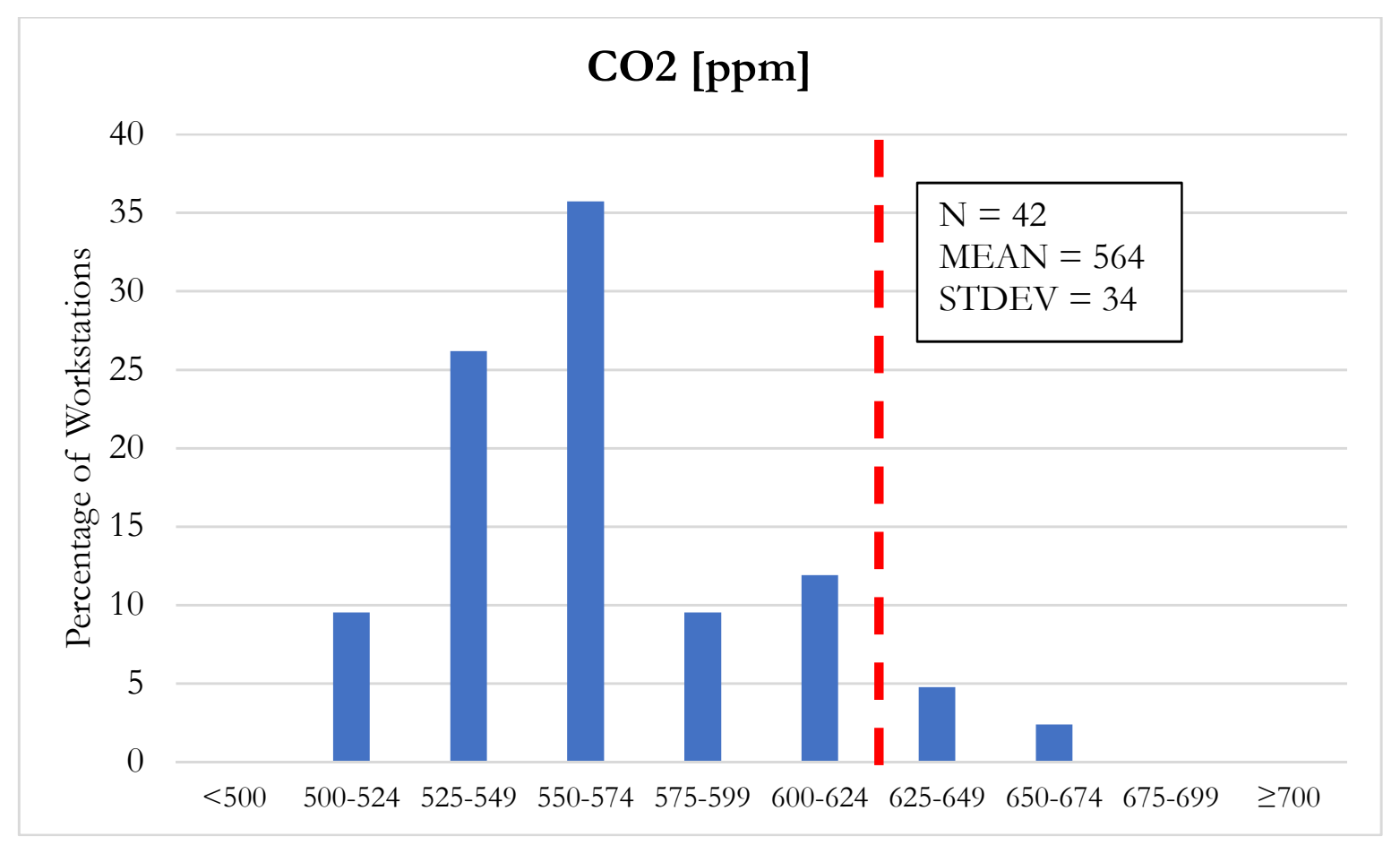

Figure 16. Carbon dioxide concentrations in workstations.

Figure 17 shows the data related to particulates concentration measured in the indoor air at the workstations. Standards and recommendations typically apply to particulates $\leq 2.5$ microns in diameter $\left(\mathrm{PM}_{2.5}\right)$ and $\leq 10$ microns in diameter $\left(\mathrm{PM}_{10}\right)$. Respirable particles in this size range have been associated with negative health outcomes (Xing, Xu, Shi, \& Lian, 2016). Due to limitations in instrumentations, only particulates $\leq 2.5$ microns were considered in this study. 
ASHRAE Standard 62.1 (2016) summarizes acceptable particulate thresholds in various jurisdictions. In the US, the most stringent regulations require $\mathrm{PM}_{2.5}$ to be less than 15 $\mu \mathrm{g} / \mathrm{m}^{3}$ for a 1-year period and less than $65 \mu \mathrm{g} / \mathrm{m}^{3}$ for a 24-hour period. In Canada, guidelines from Health Canada recommend $\mathrm{PM}_{2.5}$ concentrations to be less than $40 \mu \mathrm{g} / \mathrm{m}^{3}$ over the long term and less than $100 \mu \mathrm{g} / \mathrm{m}^{3}$ for a 1-hour period. The particulate levels measured at the workstations were well below the most stringent recommended exposure levels, with an average measured $\mathrm{PM}_{2.5}$ of $3.1 \mu \mathrm{g} / \mathrm{m}^{3}$.

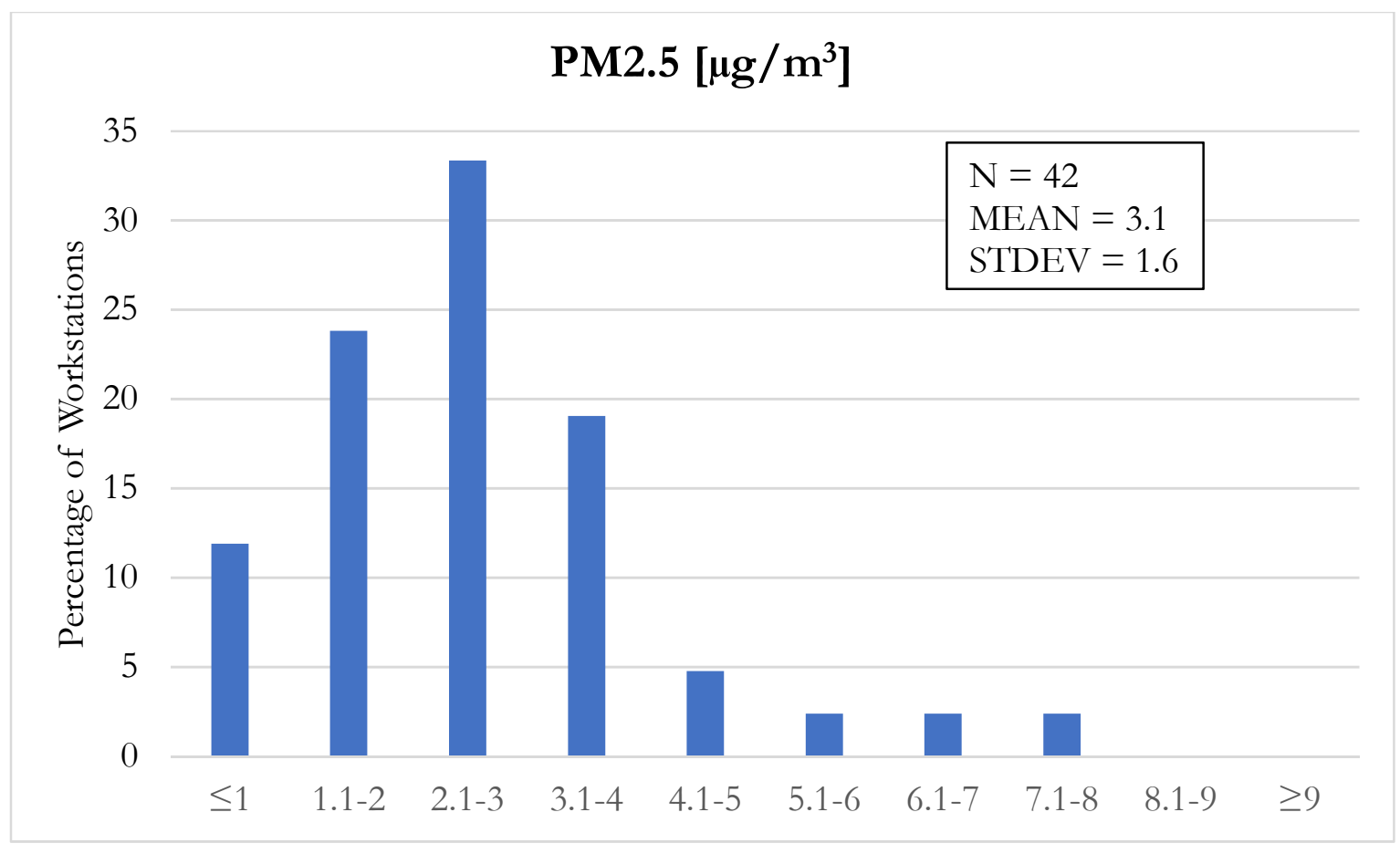

Figure 17. $\mathrm{PM}_{2.5}$ concentrations in workstations.

\subsubsection{Acoustics \& Noise}

Figures 18 below shows the aggregate survey results from Core Module \#2 regarding satisfaction with acoustics and privacy. This metric is the combined effects of workplace privacy as well as both background and speech noises.

The results of this metric indicate that occupants are generally satisfied with their workstations in terms of acoustics and privacy. The average satisfaction with acoustics and privacy was 5 , above the neutral point of the scale. Although approximately $30 \%$ of the occupants still indicated that they were slightly unsatisfied $(<3)$, no occupants responded with the worst 2 options regarding the overall acoustics and privacy of their office.

Figures 19 and 20 below show ratings for select questions in Optional Module \#1 of the survey, regarding detailed feedback on acoustics and noise. On average, respondents thought their workstations were moderately private and do not experience a lot of annoyance with speech sounds. A small number of approximately $15 \%$ of respondents felt noises due to speech was very or extremely annoying. 
Upon further review of open-ended responses regarding acoustics, it was apparent that a non-insignificant portion of the respondents had issues with speech noises from adjacent rooms and street level. These responses appeared to be more contextual in nature than to express extreme dissatisfaction, as their closed-survey responses did not indicate severe dissatisfaction with privacy and noise levels at their workstations.

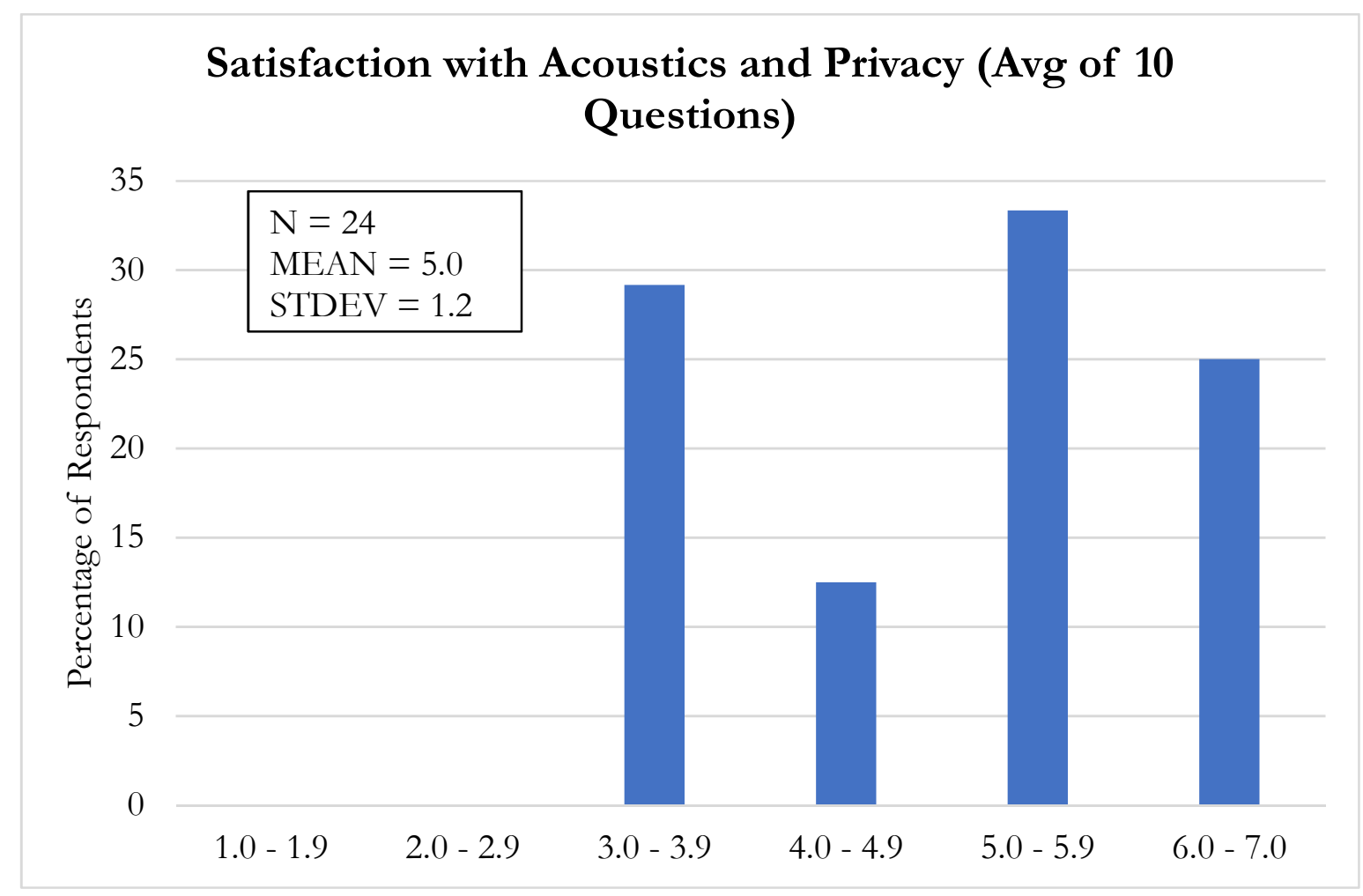

Figure 18. Satisfaction with acoustics and privacy; $1=$ very unsatisfactory, $4=$ neutral, $7=$ very satisfactory. 


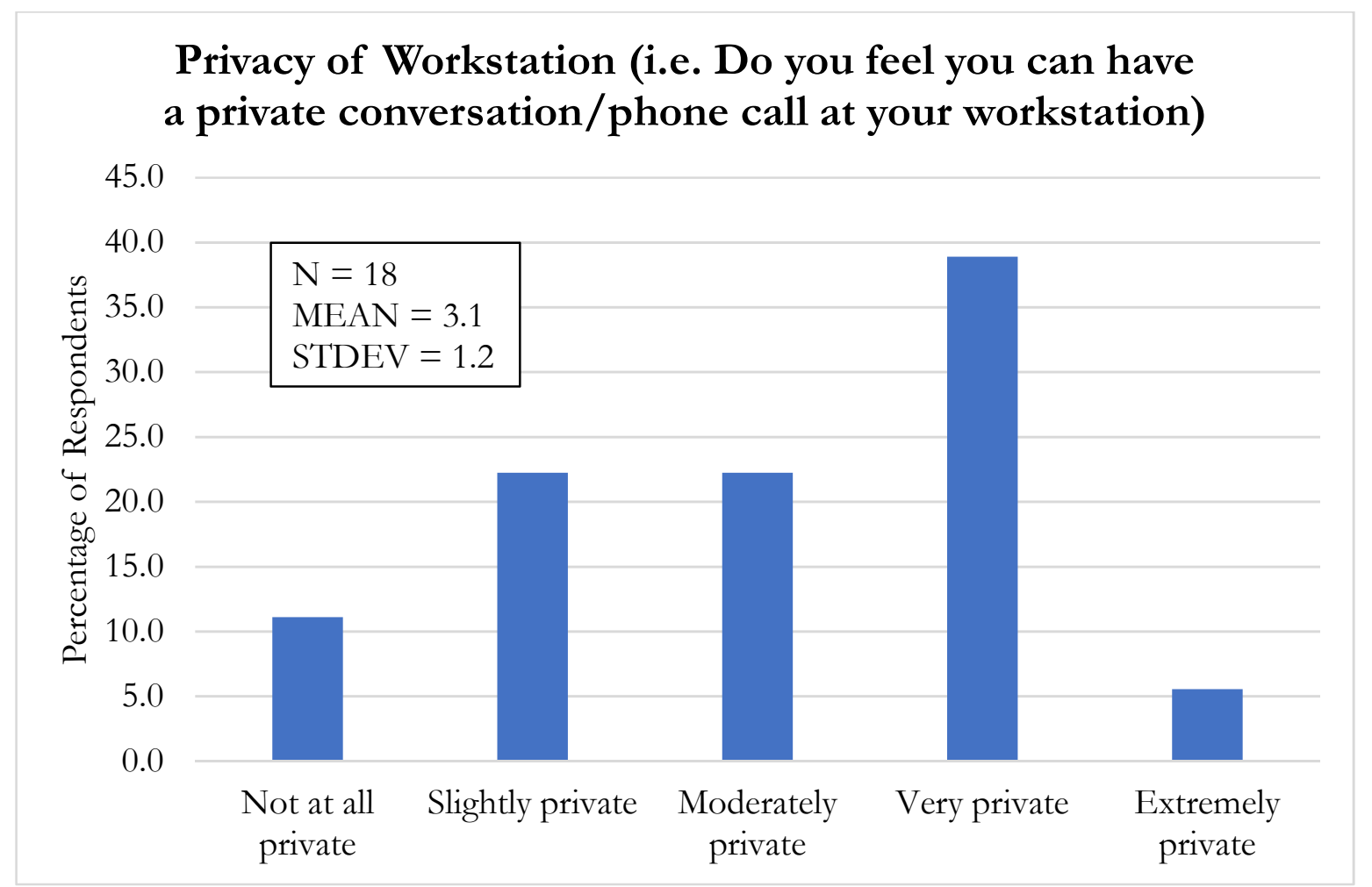

Figure 19. Privacy of workstation; $1=$ not at all private, $3=$ moderately private, $5=$ extremely private.

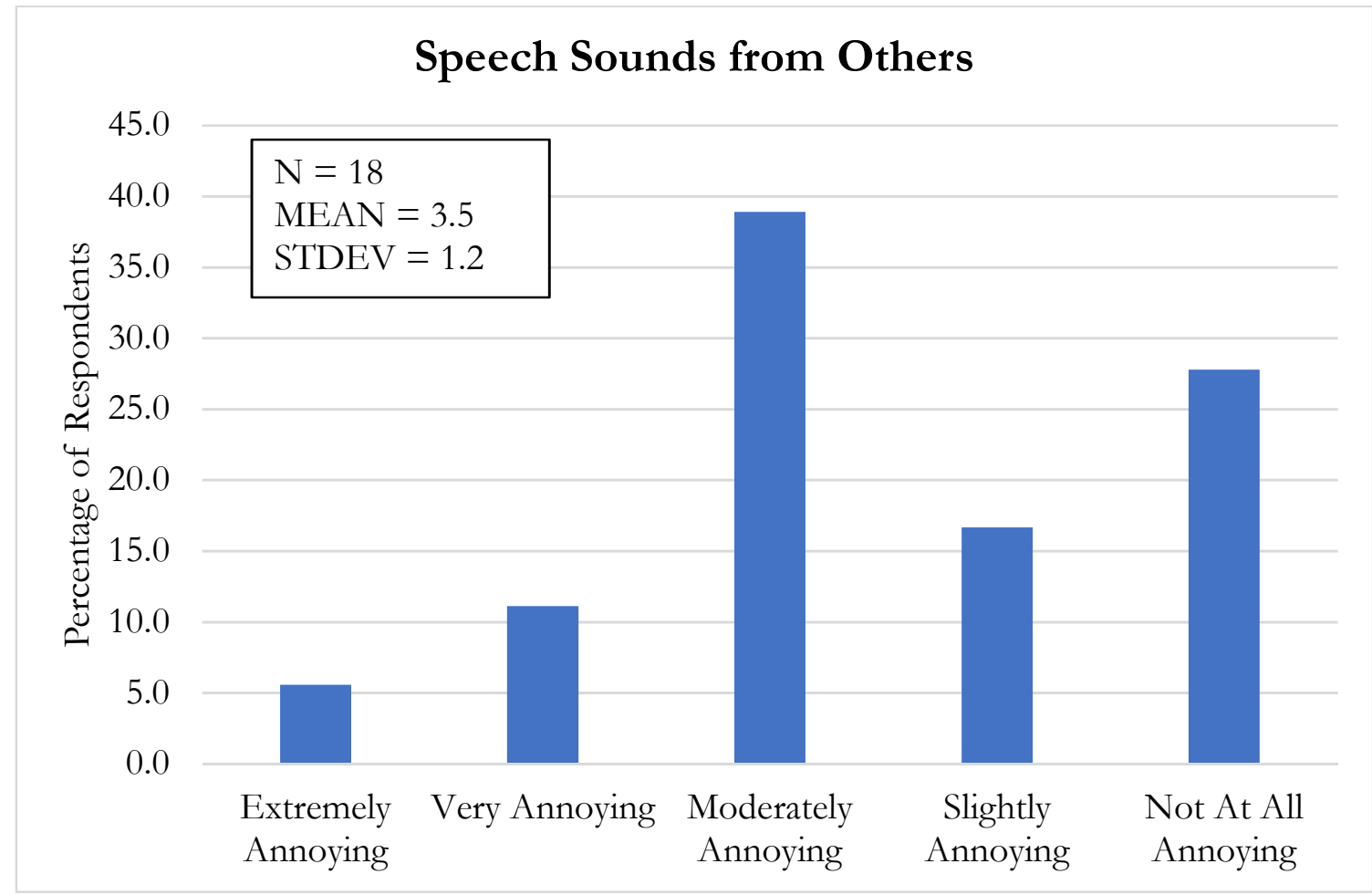

Figure 20. Speech sounds from others; $1=$ extremely annoying, $3=$ moderately annoying, $5=$ not at all annoying. 
Figure 21 shows physical measured data related to acoustics. The ambient sounds in the workstations were measured using A-weighted sound pressure levels. This is a commonly used weighting-scheme to adjust instrument measurement frequencies to account for loudness perceived by the human ear.

The ANSI/ASA S12.2 (2008] standard has recommended an A-weighted decibel of 44 to 48 dBA for office spaces with business machines (computers). Bradley \& Gover (2004) has recommended an ambient sound level high enough to provide some masking of distracting speech sounds, but not so loud that it becomes annoying in itself; they suggest $45 \mathrm{dBA}$, with 48dBA considered too high.

Approximately $65 \%$ of the measured locations were below the more stringent ambient sound level recommendation of $44 \mathrm{dBA}$, and $12 \%$ of the measured workstations exceeded the suggested ambient sound level suggestion of $48 \mathrm{dBA}$.

The data suggest that the workstations may have been a little quiet in terms of sound masking, with approximately $40 \%$ of the measured locations falling below $40 \mathrm{dBA}$. This may have negative impacts on the perception of speech sounds during non-ambient conditions (e.g. foot traffic, meetings nearby, street-level sounds). Considerations could be made regarding the implementation sound masking in the future to dull the impact of distracting speech sounds.

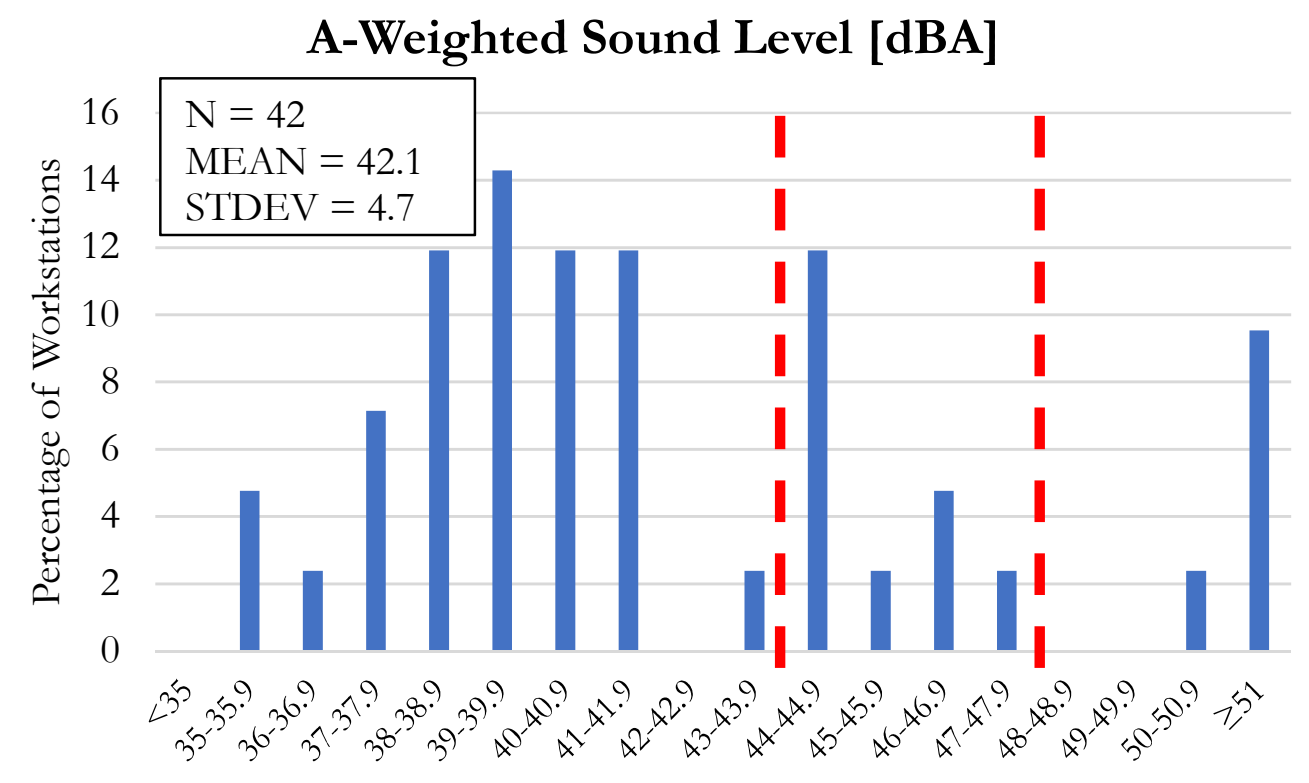

Figure 21. A-weighted sound levels at workstations.

\subsubsection{Lighting}

Figure 22 below shows the aggregate survey results from Core Module \#2 regarding satisfaction with lighting. Overall, the occupants appeared to be satisfied with the level of lighting in their workstations. The average response regarding satisfaction with lighting was 5.2 , above the neutral point of the scale. Approximately $20 \%$ of the respondents 
indicated dissatisfaction with lighting $(<3)$, though no occupants responded with the worst option. Lighting was the best performing aspect of their workstation environment (out of ventilation, thermal comfort, and acoustics) based on the perception and responses of the occupants.

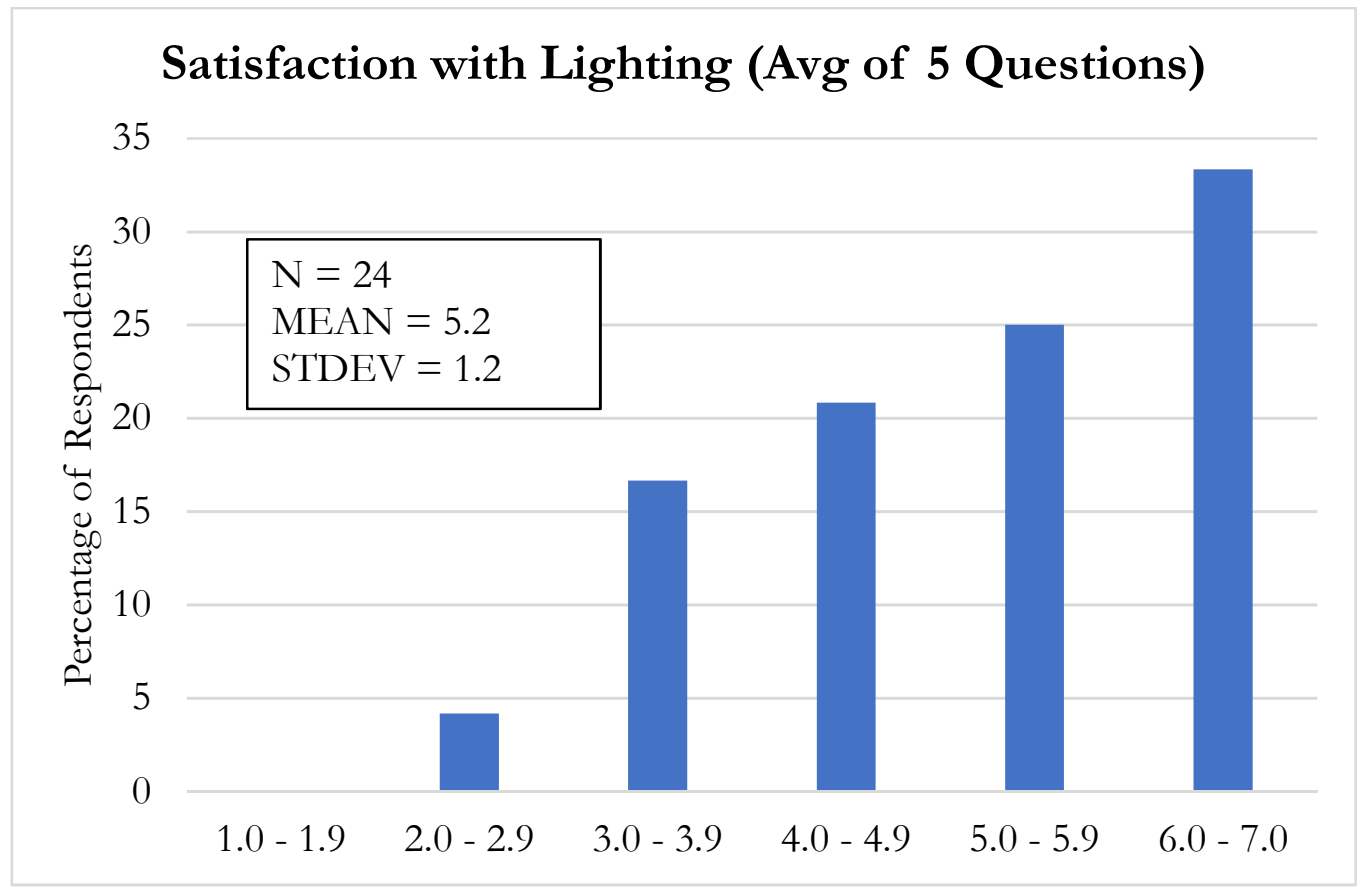

Figure 22. Satisfaction with lighting; $1=$ =very unsatisfactory, $4=$ neutral, $7=$ =very satisfactory.

Figure 23 shows the measurements of desktop illuminance with regards to lighting. This was based on the mean of two measurements made on the left and right sides of the desk where the occupant typically sits. In interpreting this data, it is important to understand the measurement conditions. At the time of measurement, the electric lighting was manually turned on via wall switches by the researcher. Task lights were left in the 'off' position in the workstations where they were present. Shading devices in offices with windows were left in the position they were found in (which varied). As such, the exterior weather conditions (sunny vs overcast) and the status of the shading devices could have influenced and/or accentuated the differences in desktop lighting levels.

As expected, light levels in locations with windows were higher on average compared to locations without windows. Of course, this comparison is dependent on factors such as the exterior conditions at the time of the site visit and the time of day of individual measurements in relation to the direction the window faced.

With reference to Canada Labour Code ${ }^{1}$ and ANSI/IESNA (2012), desktop illuminances in offices are recommended to be in the range $300-500 \mathrm{~lx}$ for general office work, and less than $500 \mathrm{~lx}$ for computer-based (VDT ${ }^{2}$ ) work.

\footnotetext{
${ }^{1}$ See: http://laws.justice.gc.ca/eng/regulations/SOR-86-304/FullText.html, Part VI.

${ }^{2}$ Visual display terminal
} 
In terms of illuminance, the median is often a better indication of central tendency than the mean, due to the potential for very high values close to sunny windows to bias the mean. The median value measured in the workstations was 398 lux, which is within the illuminance recommendation limits for office spaces; approximately $40 \%$ of the measured spaces was within these limits. Around $26 \%$ of the workstations had lighting below recommended levels. Considerations could be made regarding increasing the amount of lighting, along with adjustable lighting controls and/or shading devices for occupants who prefer dim environments.

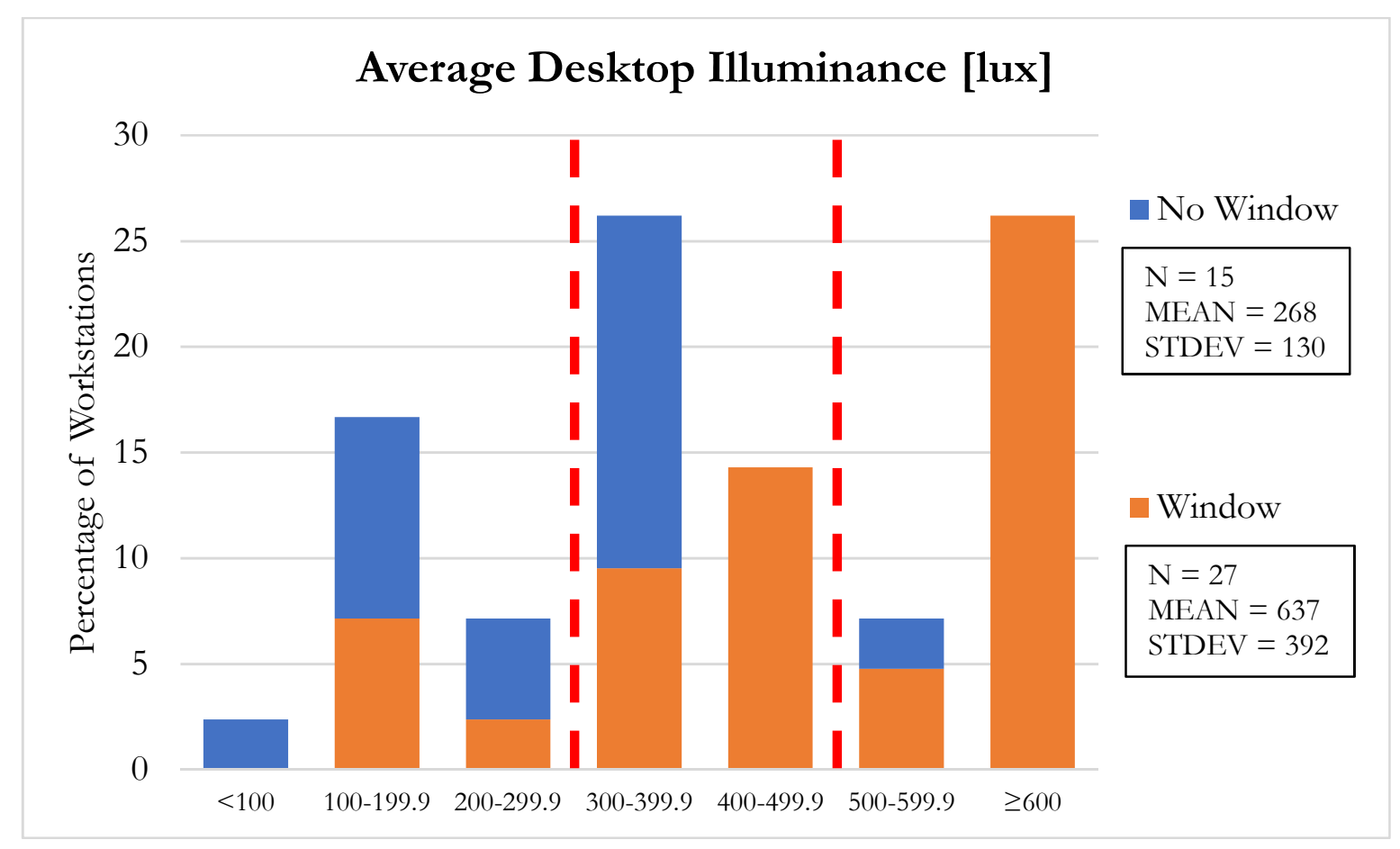

Figure 23. Desktop illuminance at workstations.

\subsubsection{Overall Environment Satisfaction}

Figure 24 below shows the aggregate survey results from Core Module \#2 regarding overall environment satisfaction. This measure was developed by (J.A. Veitch et al., 2003) to evaluate the overall satisfaction of occupants with their work environment. It combines the influences of individual environmental factors (lighting, acoustics \& privacy, thermal \& ventilation) as well as workstation characteristics.

In general occupants appeared to be satisfied with the overall conditions of their workstations. The average reported score of overall environment satisfaction was 4.8, which is above the neutral point of the scale. Additionally, more than one-third of the respondents deemed their overall work environment to be in the highest satisfaction category. However, this is accompanied by the fact that approximately $30 \%$ of the respondents rated their overall environment unfavourably and below the neutral point of the scale $(<3)$. 


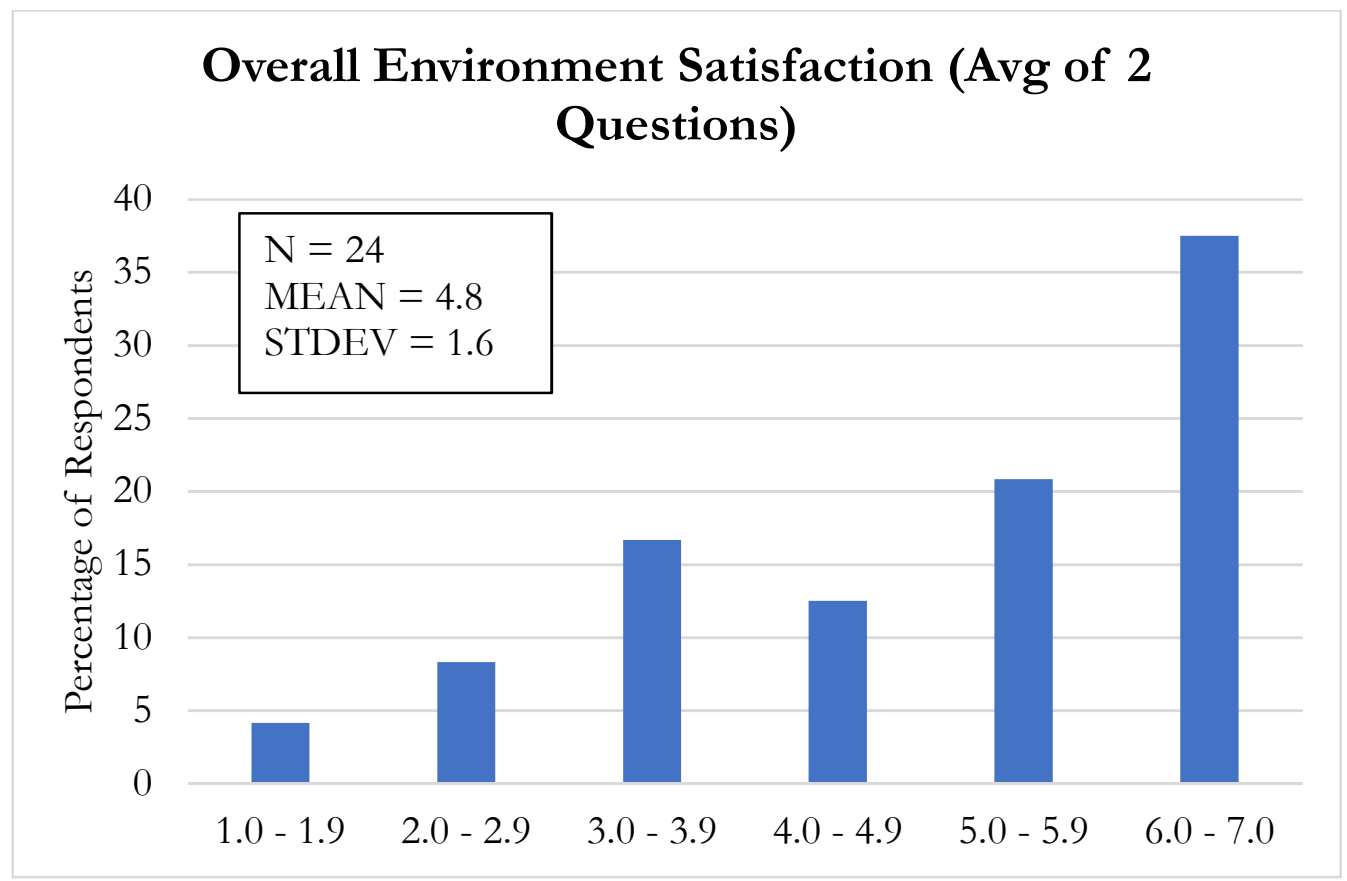

Figure 24. Overall environmental satisfaction; $1=$ =very unsatisfactory, $4=$ =neutral, $7=$ =very satisfactory.

\subsection{Workplace Productivity}

\subsubsection{Environmental Effect on Productivity}

Figure 25 below shows the distribution of the responses from Core Module \#3 regarding the overall effects of the indoor environment on productivity. This metric incorporates the perceptions of the effects of temperature and humidity, air quality, illuminance, and noise levels. The average response for this variable was 3.0, at the neutral point of the scale. Only approximately $20 \%$ of the respondents felt that the indoor environment affected their productivity in an 'extreme' way (score of either 1 or 5). This indicates that although the indoor environment plays a role in terms of impacting productivity, most occupants did not feel like it had a large effect.

An overall environmental factor score (out of 100) was calculated based on the responses (Carey et al., in press), where a score of 50 indicates no effect on productivity (neutral), and scores above and below 50 represent positive and negative effects, respectively. The resulting score determined was 46.67 . This shows that improvements can be made regarding the indoor environment (refer to Section 3.2 for lacking areas) to improve occupant productivity and performance. 


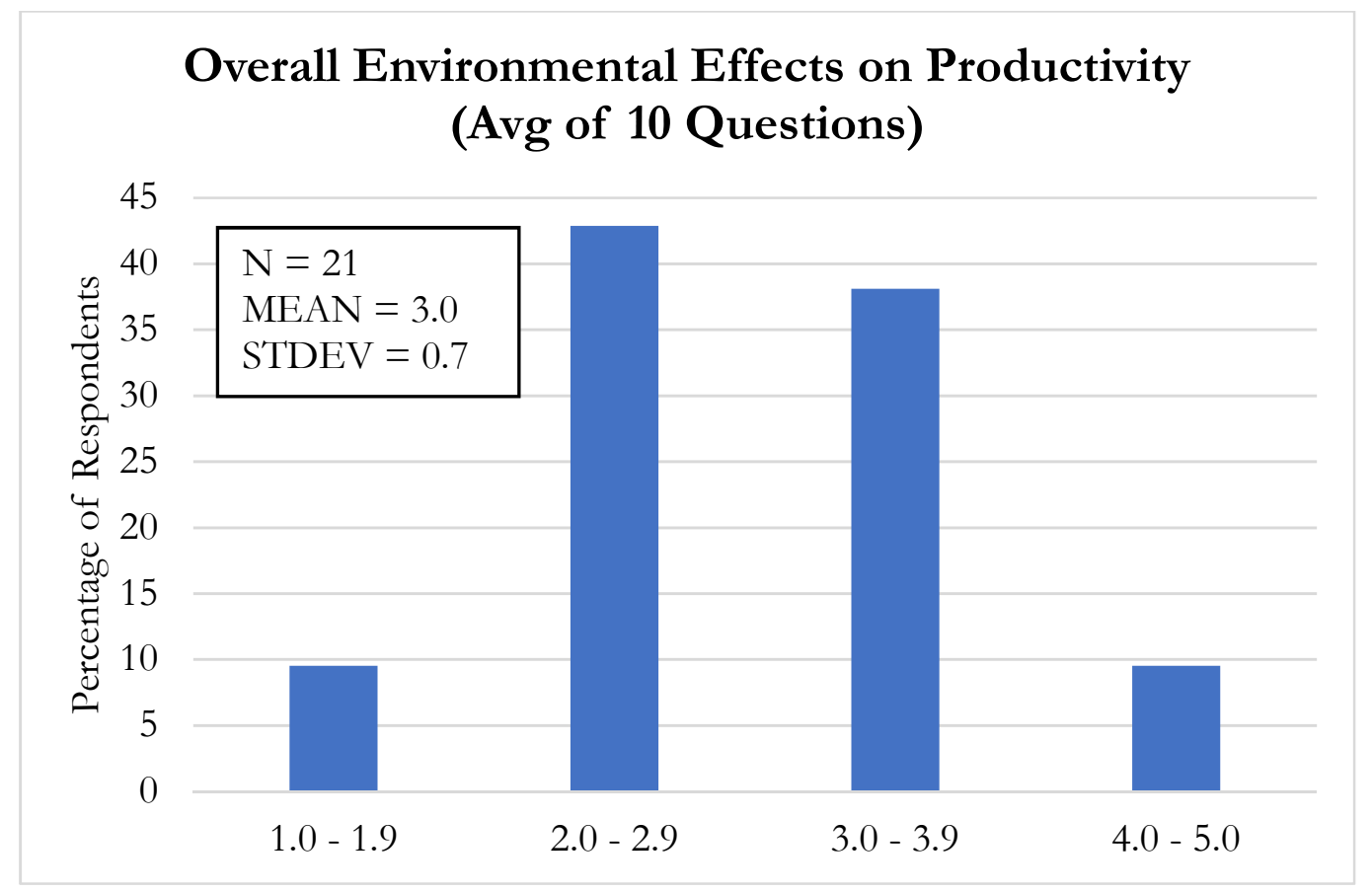

Figure 25. Overall environmental effect on productivity; 1 = extremely detrimental, 3 = no effect, 5 = extremely beneficial.

\subsubsection{Health Effect on Productivity}

Figure 26 below shows the distribution of the responses from Core Module \#3 regarding the overall effects of occupant health on productivity. This metric evaluates the effects of the occupant health on factors such as overall productivity, ability to complete tasks to usual ability, ability to take pleasure in work etc.

The average response for this variable was 3.0, at the neutral point of the scale. Only approximately $30 \%$ of the respondents felt that health affected their productivity in an 'extreme' manner (score of either 1 or 5). Like environmental effects, this indicates that most respondents did not feel that their health had a large impact on their productivity and performance in the workplace.

An overall health factor score (out of 100) was calculated based on the responses, where a score of 50 indicates no effect on productivity (neutral), and scores above and below 50 represent positive and negative effects, respectively. The resulting score determined was 49.21. This illustrates that improvements can be made regarding the impacts of occupant health and subsequently their productivity at work. 


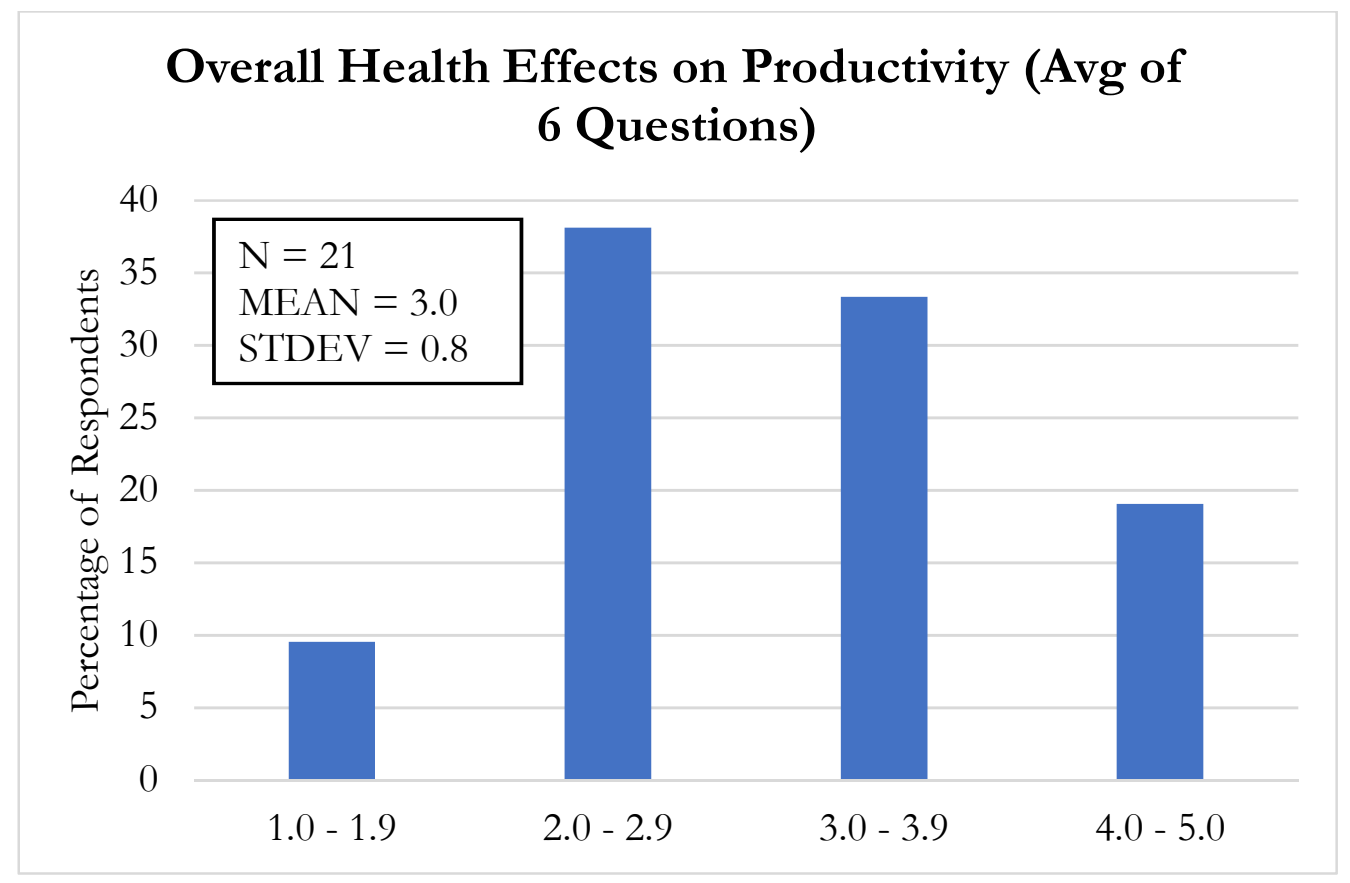

Figure 26. Overall health effect on productivity; 1 = extremely detrimental, $3=$ no discernible effect, $5=$ extremely beneficial.

\subsubsection{Behaviour Effect on Productivity}

Figure 27 below shows the distribution of the responses from Core Module \#3 regarding the overall effects of occupant behaviour on productivity. This metric evaluates behavioural factors such as unplanned interactions with coworkers, office morale, as well as relationships with peers, supervisors, and direct reports etc.

The average response for this variable was 2.8, slightly below the neutral point of the scale. Approximately $10 \%$ of respondents felt that behavioural aspects effected their productivity in an 'extreme' manner (score of either 1 or 5 ). Like the previously discussed productivity metrics, this shows that most respondents did not feel that behavioural factors had a large impact on their workplace productivity. In addition, no respondents felt that behavioural factors had an extreme positive effect on their productivity, contrasted with the $10 \%$ of responses that reported extreme negative effects.

An overall behaviour factor score (out of 100) was calculated based on the responses, where a score of 50 indicates no effect on productivity (neutral), and scores above and below 50 represent positive and negative effects, respectively. The resulting score determined was 28.57. This indicates that behavioural factors are an area of weakness, and that improving the interactions between colleagues can have benefits to employee productivity in the workplace. 


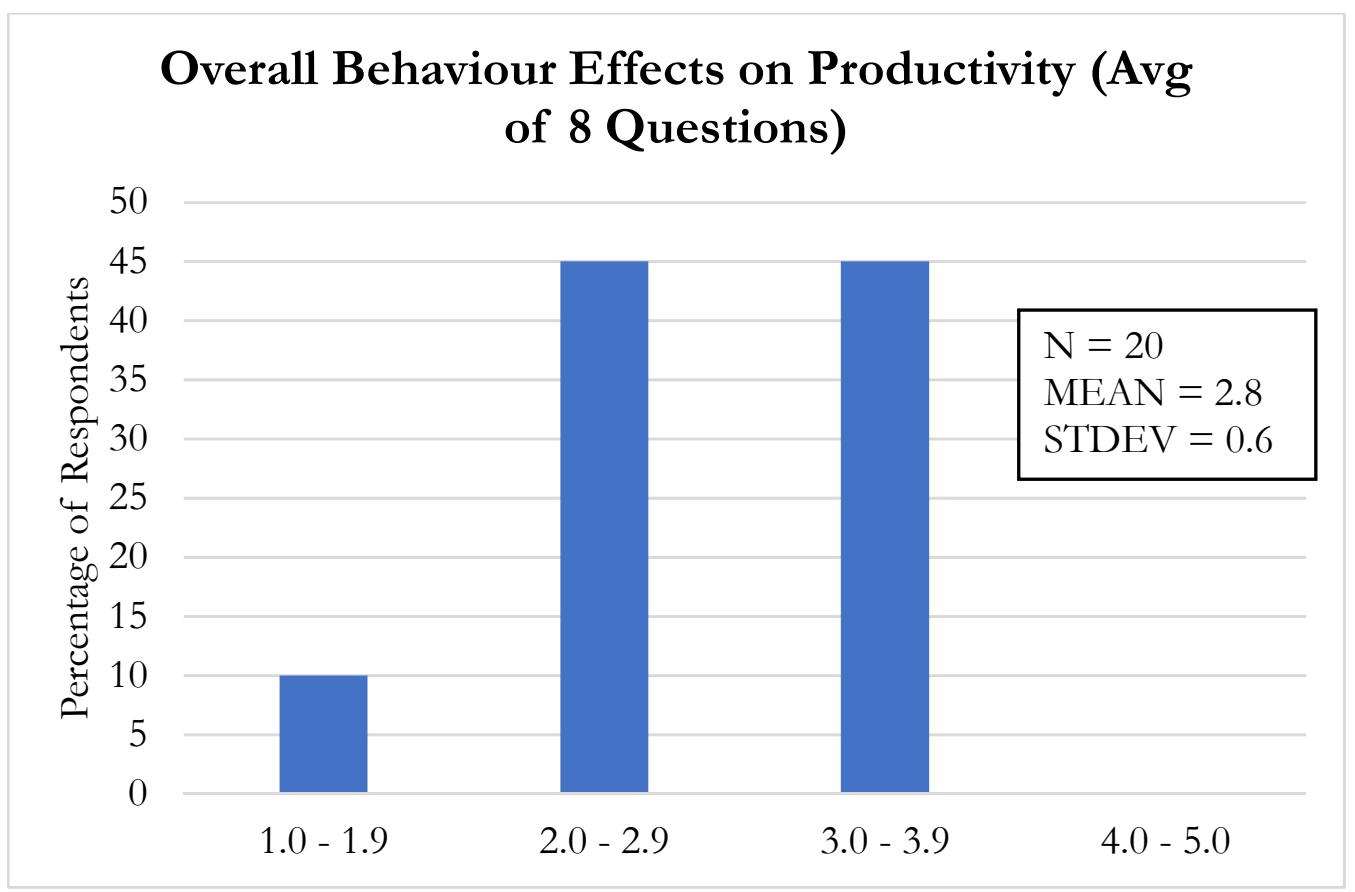

Figure 27. Overall behaviour effect on productivity; 1 = extreme negative effect, 3 = no effect, $5=$ extreme positive effect.

\subsubsection{Organizational Effect on Productivity}

Figure 28 below shows the distribution of the responses from Core Module \#3 regarding the overall organizational effects on productivity. This metric evaluates organizational factors such as office layout, organizational management style, organizational hierarchy, and integration between teams.

The average response for this variable was 3.0, at the neutral point of the scale. The results show that approximately $20 \%$ of respondents felt that organizational factors had an 'extreme' effect (score of either 1 or 5) on their productivity. Similar to the previous productivity metrics, this again shows that the majority of respondents did not feel that organizational factors had a large impact on their productivity in the workplace.

An overall organizational effect score (out of 100) was calculated based on the responses, where a score of 50 indicates no effect on productivity (neutral), and scores above and below 50 represent positive and negative effects, respectively. The resulting score determined was 45.24. Improvements can be made in the area of organizational factors to improve employee productivity. 


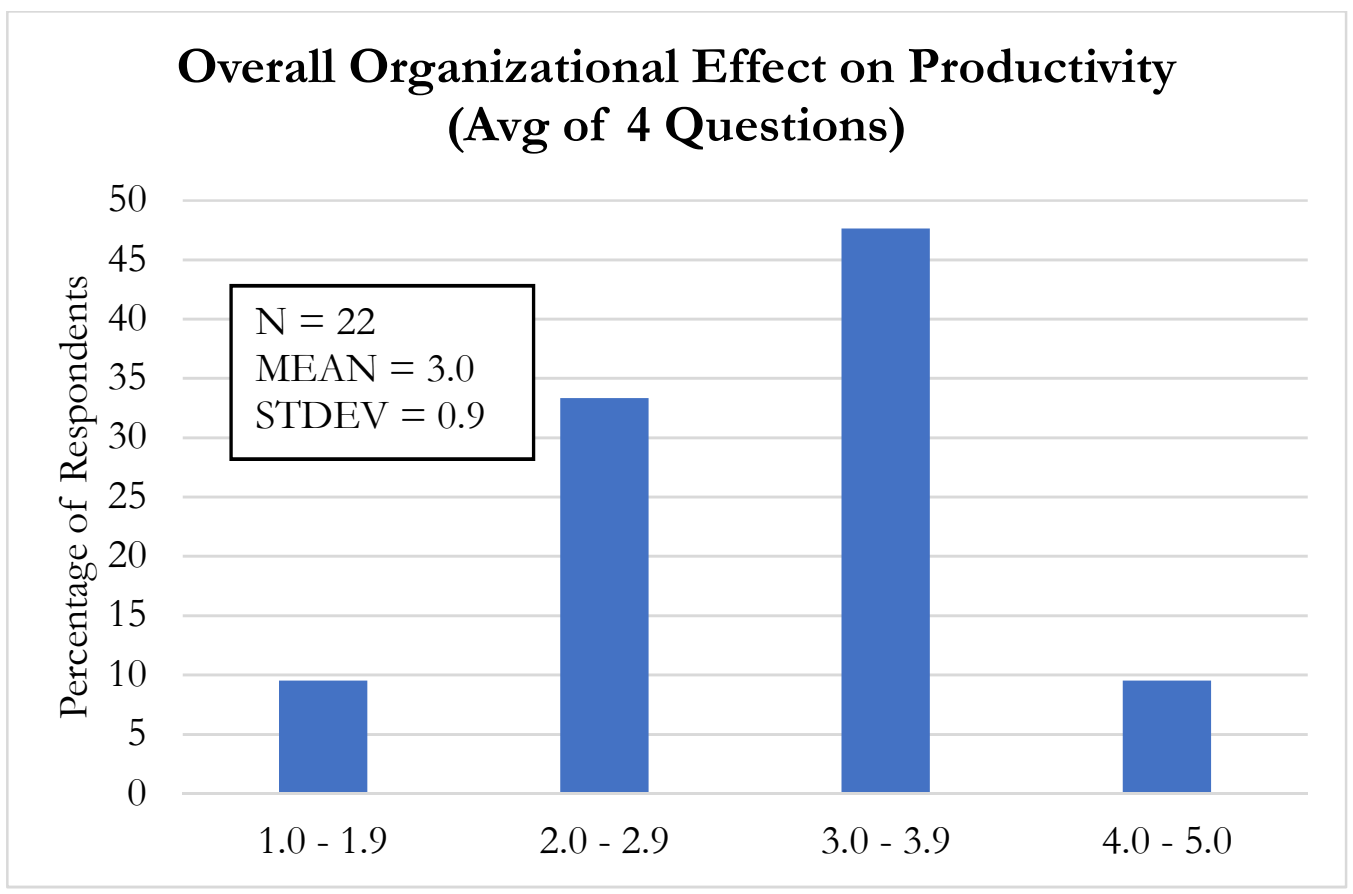

Figure 28. Overall organizational effect on productivity; $1=$ extremely detrimental, $3=$ no effect, $5=$ extremely beneficial.

\subsubsection{Work Engagement}

Figure 29 below shows the distribution of the responses from Core Module \#3 regarding the overall work engagement. This metric evaluates engagement factors such as vigor, dedication, and absorption, which, combined forms the overall work engagement metric. The results of work engagement are reported in terms of frequency (i.e. how often the occupants feel engaged in their work).

The average response for this variable was 6.0, significantly above the neutral point of the scale. The results show that occupants are very engaged in their work overall, with approximately $90 \%$ of respondents feeling engaged 'once a week' (score of 5.0) or more on average. Furthermore, only $5 \%$ of responses were below the neutral point of the scale, and none in the worst two options. These results illustrate that work engagement is an area of strength in the respondents, and that they are very engaged in their work. 


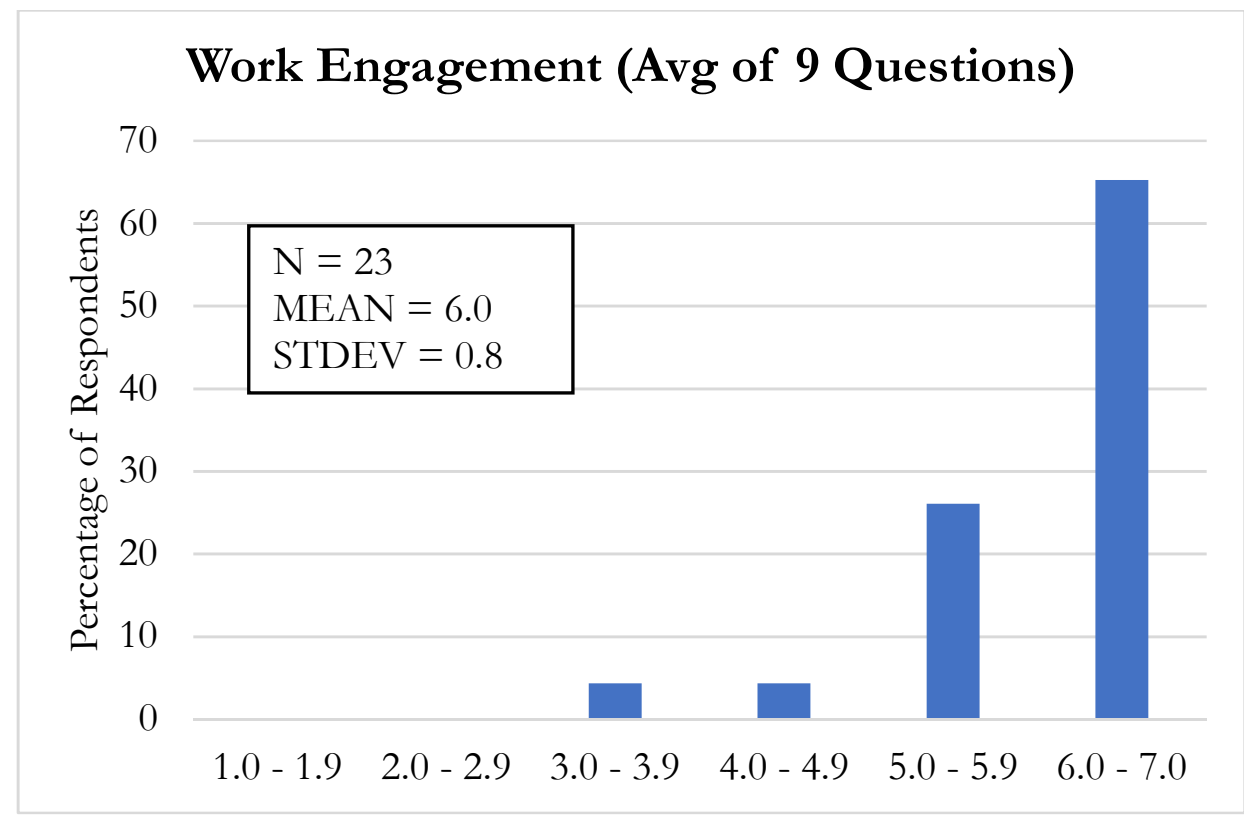

Figure 29 . Work engagement; 1 = never, 5 = sometimes (few times a month), 7 = always (everyday).

\subsubsection{Quality and Volume of Work}

Figures 30 and 31 below show the distribution of the responses from Core Module \#3 regarding the self-assessed quality and quantity of work compared to organizational expectations.

The average response for this variable was 4.0 for quality of work and 3.6 for volume of work, both above the neutral point of the scale. This is to be expected, as employees rarely rate their performance as subpar or below average. This is evident by the results, as no occupant responses were below the neutral point ('at expected standard') of the scale. 


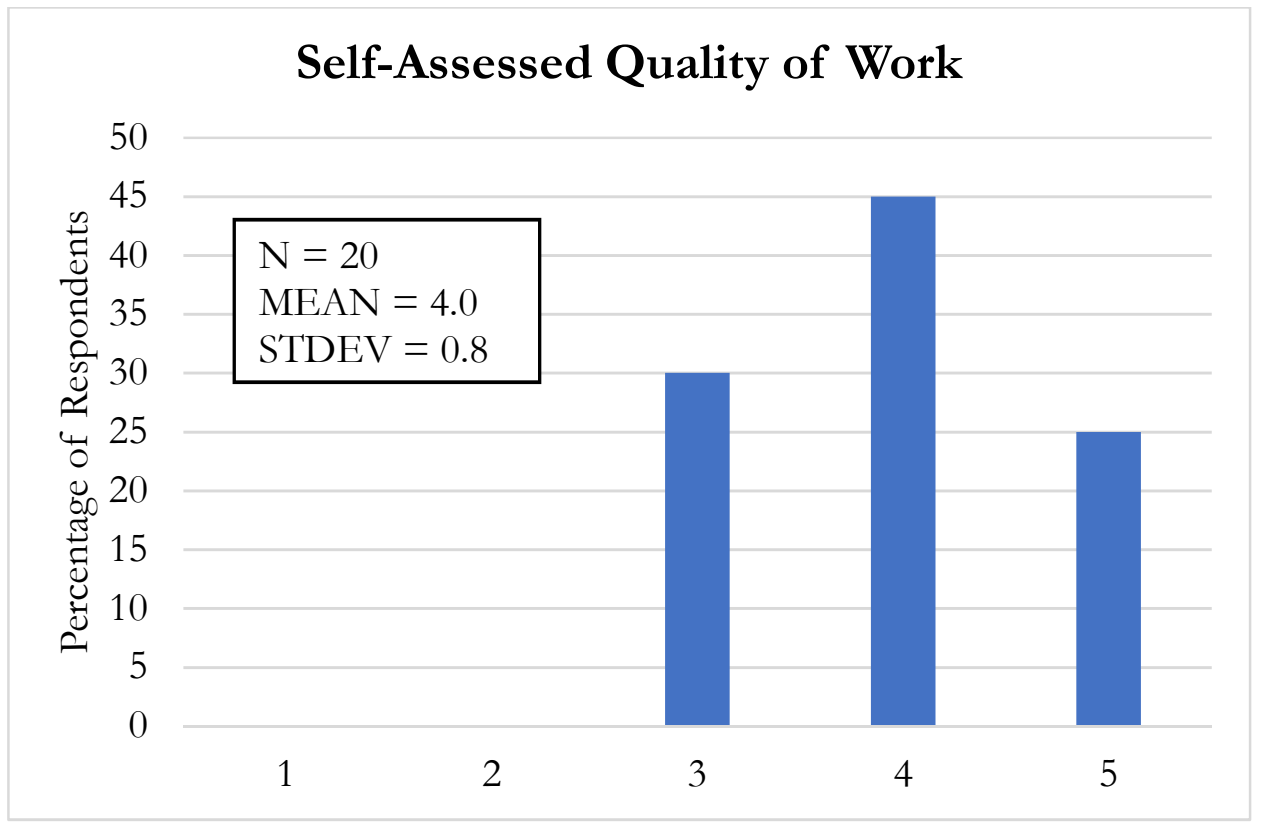

Figure 30. Self-assessed quality of work; 1 = far below the expected standard, 3 = at expected standard,

$5=$ far above the expected standard.

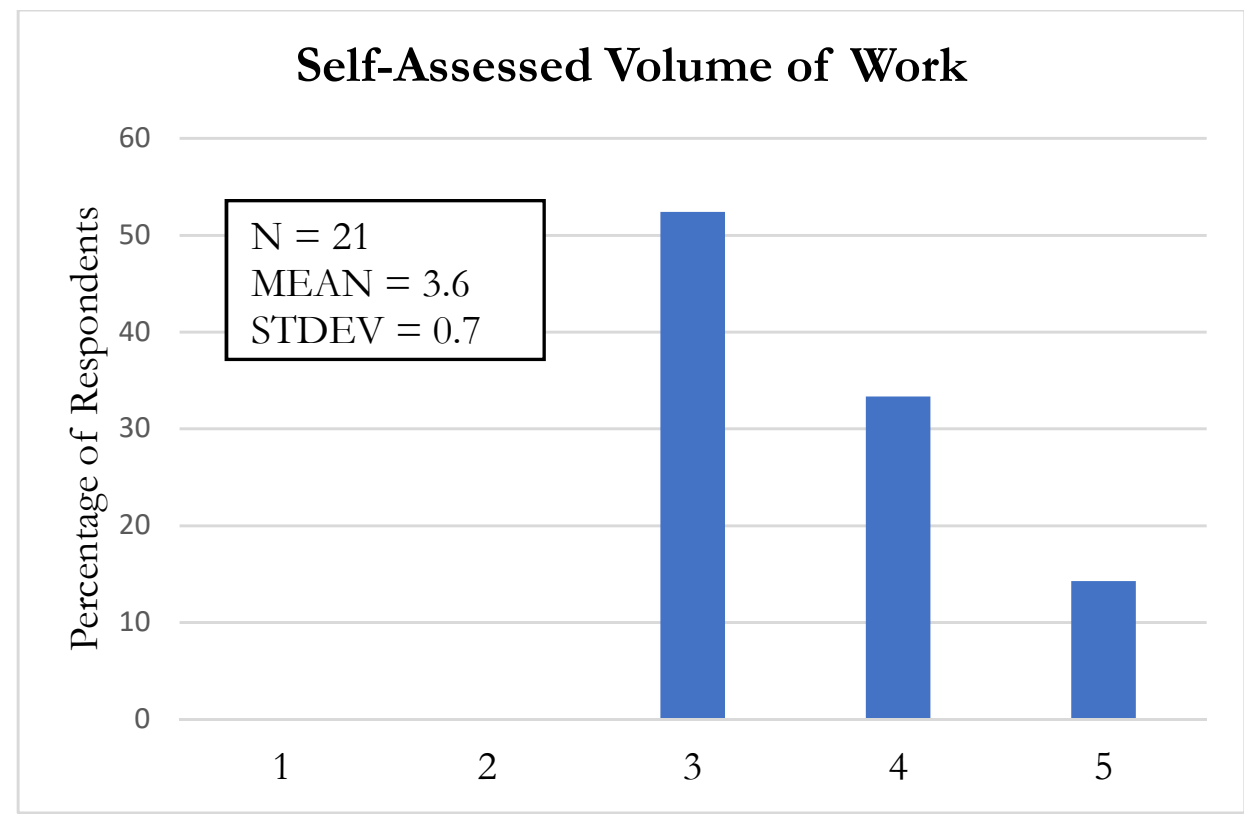

Figure 31. Self-assessed volume of work; 1 = far below the expected standard, 3 = at expected standard, $5=$ far above the expected standard. 


\subsection{Workplace Functionality, Safety, and Accessibility}

\subsubsection{Workplace Functionality}

Figure 32 below shows the aggregate survey results from Core Module \#4 regarding satisfaction with workplace functionality. This metric takes into consideration elements in the workplace such as cleanliness, places to eat and socialize, IT connectivity, meeting rooms etc.

In general, occupants viewed their workplace functionality somewhat negatively. The average reported score was 2.7, below the neutral point of the scale. Furthermore, no occupants felt very satisfied in terms of their workplace functionality, suggesting that this is an area of concern and should be improved upon.

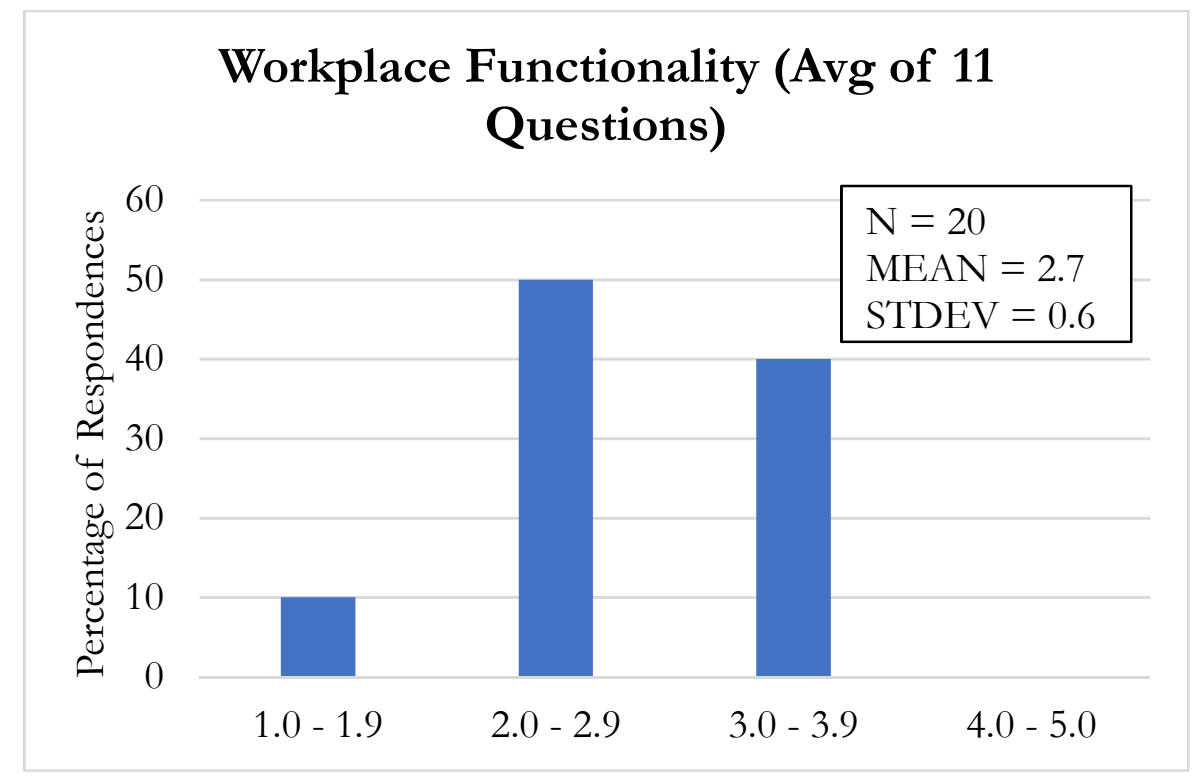

Figure 32 . Workplace functionality; $1=$ very unsatisfied, $3=$ no opinion, $5=$ very satisfied.

\subsubsection{Workplace Safety}

Figure 33 below shows the aggregate survey results from Core Module \#4 regarding satisfaction with safety. This metric takes into consideration factors such as safety at the workspace as well as approaching/leaving the building.

In general, respondents viewed their safety somewhat positively. The average reported score was 3.4 , above the neutral point of the scale. However, approximately $25 \%$ of the occupants felt their environment was unsafe, with $5 \%$ of those feeling very unsafe. Although not an overwhelming number of occupants felt this way, safety should be a priority. As such, feedback should be gathered from the occupants to inquire as to the sources of unsafe feelings, and measures implemented to address them. 


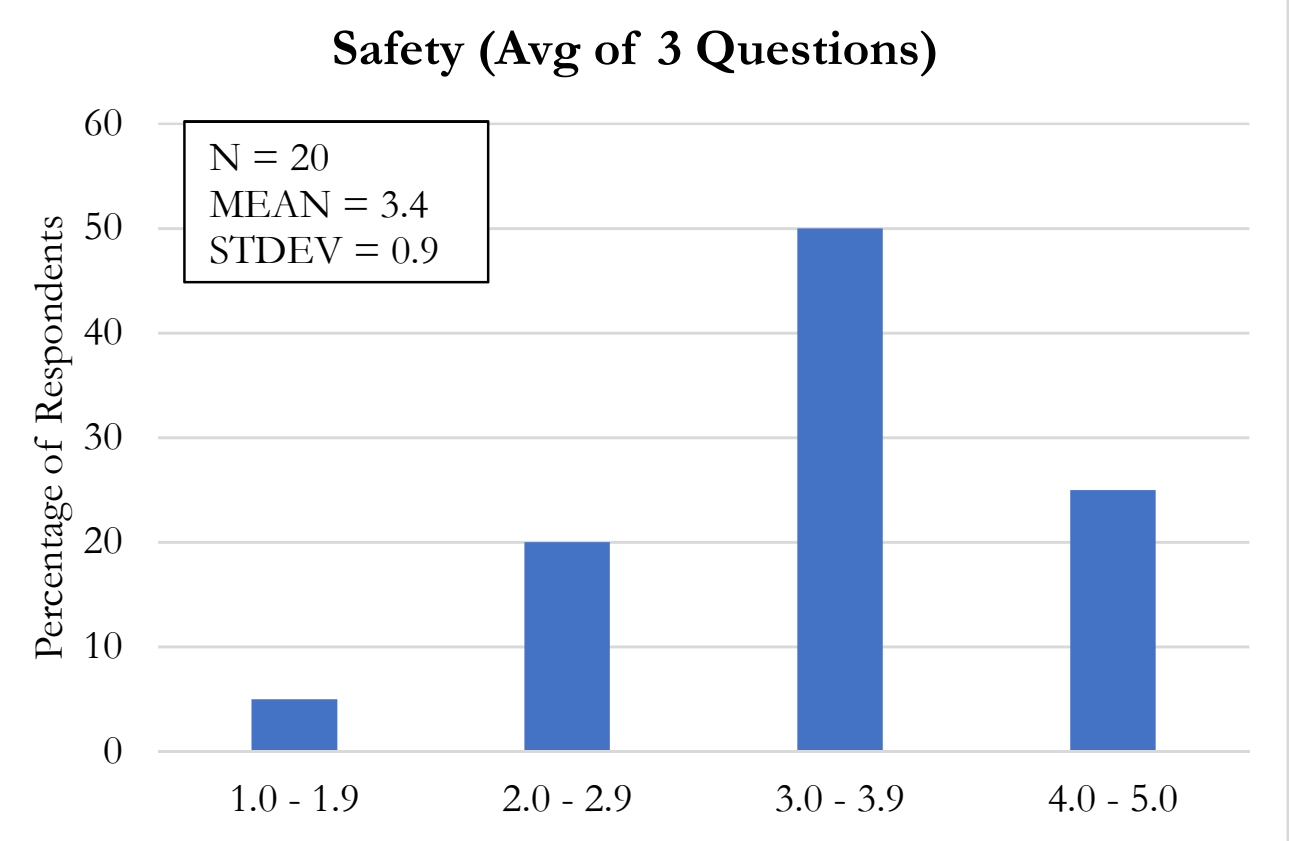

Figure 33. Safety; $1=$ very unsafe, $3=$ neither safe nor unsafe, $5=$ =very safe.

\subsubsection{Workplace Accessibility}

Figure 34 below shows the aggregate survey results from Core Module \#4 regarding satisfaction with accessibility.

In general, respondents were not satisfied with the accessibility of the buildings. The average reported score was 2.6, below the neutral point of the scale. From the results it can be observed that most occupants were impartial to the level of accessibility, approximately $60 \%$ of the responses were 'typical'. However, no occupants felt that accessibility was 'extremely good', and only 5\% of responses were positive. Furthermore, 35\% of occupants were not satisfied with accessibility, with $10 \%$ feeling that accessibility was 'extremely poor'.

Upon review of the open-ended responses, it appears that the causes of poor accessibility are varied. Occupants were dissatisfied with issues such as elevators, accessibility on weekends, signage, and traveling between buildings on campus. The issues were not just directed at the occupants' specific buildings, but some were also direct towards the Ryerson University campus in general. 


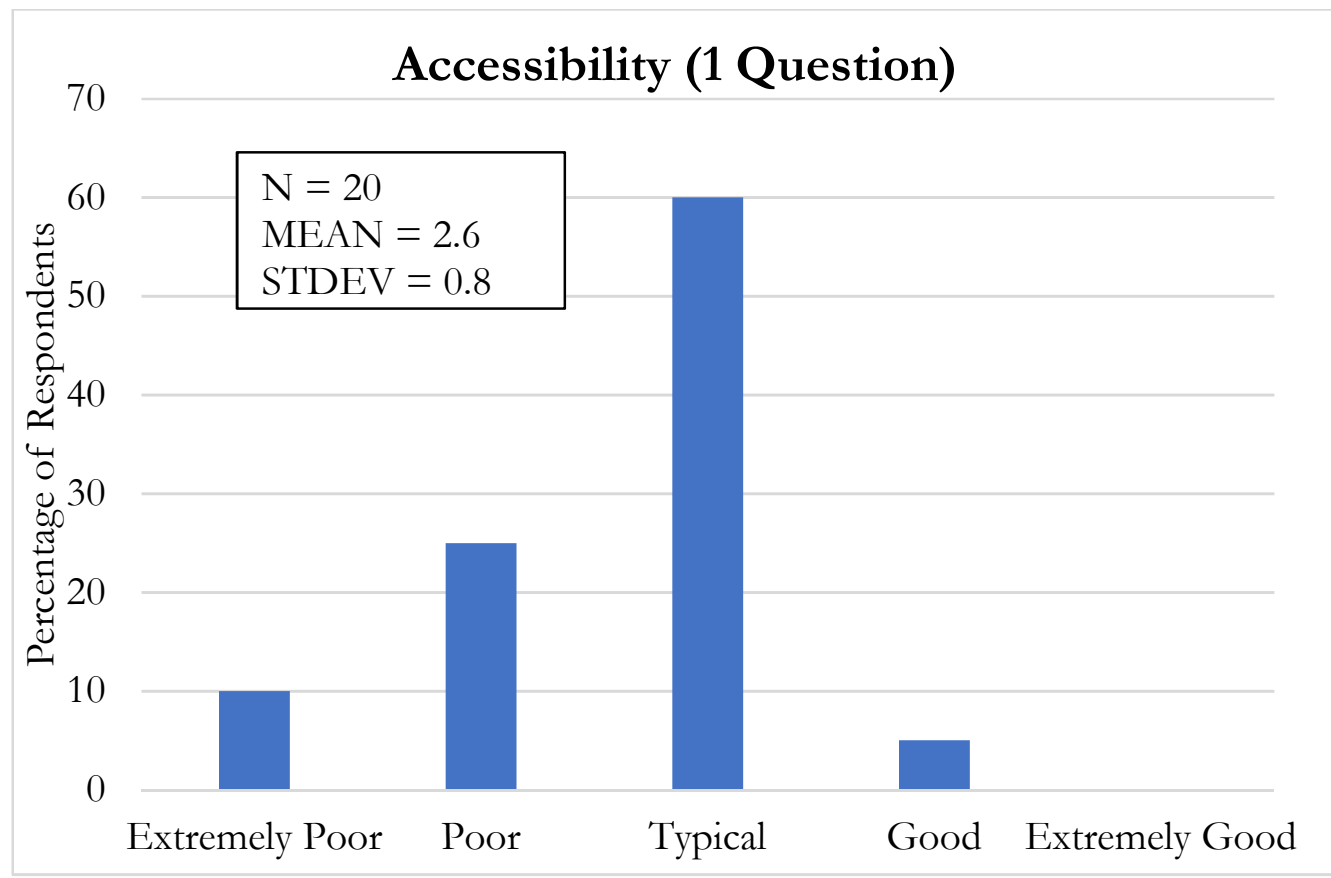

Figure 34. Accessibility of building; 1=extremely poor, 3=typical, 5=extremely good.

\section{Acknowledgements}

The research team has received tremendous support from Facilities Management \& Development as well as the Schools of Nursing, Nutrition, Occupational \& Public Health, and Midwifery Education.

We thank the faculty and staff who took time out of their busy day to participate in the physical measurements and/or questionnaire. We are also grateful to Janis Higgins-Tramov (Nursing), Julie Cabanatan (Midwifery), Clare Lalonde (Nutrition), and Darlene Bileckyj $(\mathrm{OPH})$ for coordinating the access of the offices of faculty and staff.

Finally, we would like to acknowledge the National Research Council Canada - Institute for Research in Construction for granting permission to use parts of their building evaluation questionnaire.

\section{References}

ANSI/ASA (2008). ASA S12.2 American National Standard Criteria for Evaluating Room Noise. American National Standards of the Acoustical Society of America: Melville, New York.

ANSI/IESNA. (2012). RP-1-12 American National Standard Practice for Office Lighting. Illuminating

Engineering Society of North America: New York, USA.

Apte, M. G., Fisk, W. J., \& Daisey, J. M. (2000). Associations Between Indoor C02

Concentrations and Sick Building Syndrome Symptoms in U.S. Office Buildings: An 
Analysis of the 1994-1996 BASE Study Data. Indoor Air, 10(4), 246-257. https://doi.org/10.1034/j.1600-0668.2000.010004246.x

ASHRAE. (2017) ASHRAE/ANSI Standard 55-2017 Thermal environmental conditions for human occupancy. American Society of Heating, Refrigerating, and Air-Conditioning Engineers: Atlanta, GA.

ASHRAE. (2016). ANSI/ASHRAE Standard 62.1-2016. Ventilation for acceptable indoor air quality. American Society of Heating, Refrigerating, and Air-Conditioning Engineers, Inc.: Atlanta, GA.

Bradley, J.S. \& Gover, B.N. (2004). Criteria for acoustic comfort in open-plan offices. In: Proceedings of Inter Noise 2004, Prague.

Brookes, M. J., \& Kaplan, A. (1972). The office environment: space planning and effective behavior. Human Factors, 14(5), 373-391.

Carey, D., Bortoluzzi, B., \& McArthur, J. J. (in press). A Multi-Dimensional Approach to Evaluating Productivity within the Knowledge Worker Context. Transdisciplinary Workplace Research Conference.

Choi, J. H., Loftness, V., \& Aziz, A. (2012). Post-occupancy evaluation of 20 office buildings as basis for future IEQ standards and guidelines. Energy and Buildings, 46, 167-175. https://doi.org/10.1016/j.enbuild.2011.08.009

Leder, S., Newsham, G. R., Veitch, J. A., Mancini, S., \& Charles, K. E. (2016). Effects of office environment on employee satisfaction: A new analysis. Building Research and Information, 44(1), 34-50. https://doi.org/10.1080/09613218.2014.1003176

McIntyre, D. A. (1978). Response to Atmospheric Humidity at Comfortable Air Temperature: A Comparison of Three Experiments. The Annals of Occupational Hygiene, 21(2), 177-190. https://doi.org/10.1093/annhyg/21.2.177

Newsham, G. R., Birt, B. J., Arsenault, C., Thompson, A. J. L., Veitch, J. A., Mancini, S., ... Burns, G. J. (2013). Do 'green' buildings have better indoor environments? New evidence. Building Research \& Information, 41(4), 415-434. https://doi.org/10.1080/09613218.2013.789951

Newsham, G. R., Veitch, J. A., \& Charles, K. E. (2008). Risk factors for dissatisfaction with the indoor environment in open-plan offices: An analysis of COPE field study data. Indoor Air, 18(4), 271-282. https://doi.org/10.1111/j.1600-0668.2008.00525.x

Seppanen, O. A., Fisk, W. J., \& Mendell, M. J. (1999). Association of Ventilation Rates and CO2 Concentrations with Health and Other Responses in Commercial and Institutional Buildings. Indoor Air, 9(4), 226-252. https://doi.org/10.1111/j.16000668.1999.00003.x

Veitch, J. A., Charles, K. E., Newsham, G. R., Marquardt, C. J. G., \& Geerts, J. (2003). Environmental Satisfaction in Open-Plan Environments: 5. Workstation and Physical 
Condition Effects. IRC-RR-154. National Research Council, Ottawa, ON.

https://doi.org/10.4224/20386149

Xing, Y. F., Xu, Y. H., Shi, M. H., \& Lian, Y. X. (2016). The impact of PM2.5 on the human respiratory system. Journal of Thoracic Disease, 8(1), E69-E74.

https://doi.org/10.3978/j.issn.2072-1439.2016.01.19

Yildirim, K., Akalin-Baskaya, A., \& Celebi, M. (2007). The effects of window proximity, partition height, and gender on perceptions of open-plan offices. Journal of Environmental Psychology, 27(2), 154-165.

https://doi.org/10.1016/j.jenvp.2007.01.004 


\section{APPENDIX B - Bivariate Correlation Tables}

B-1: Matched COPE Dataset

\begin{tabular}{|c|c|c|c|c|c|c|c|c|c|c|c|}
\hline & Age & Sex & Window & MinPH & $\begin{array}{l}\text { AveIlluD } \\
\text { esk }\end{array}$ & AW & SII & AirTemp & RH & $\begin{array}{c}\text { AirVeloci } \\
\text { ty }\end{array}$ & $\begin{array}{c}\text { RadiantT } \\
\text { emp }\end{array}$ \\
\hline Age & 1 & & & & & & & & & & \\
\hline Sex & 0.04 & 1 & & & & & & & & & \\
\hline Window & $0.19 * *$ & 0.03 & 1 & & & & & & & & \\
\hline $\mathrm{MinPH}$ & $0.16^{* *}$ & 0.01 & $0.18^{* *}$ & 1 & & & & & & & \\
\hline AveIlluDesk & $0.11 * *$ & 0.03 & $0.35^{* *}$ & -0.01 & 1 & & & & & & \\
\hline AW & $-0.15^{* *}$ & 0.00 & 0.03 & $-0.31 * *$ & -0.05 & 1 & & & & & \\
\hline SII & 0.04 & 0.05 & 0.02 & $-0.19 * *$ & $0.12 * *$ & $-0.59 * *$ & 1 & & & & \\
\hline AirTemp & $-0.11 * *$ & $0.12 * *$ & $-0.20 * *$ & $-0.26^{* *}$ & 0.00 & $0.11 * *$ & $0.13 * *$ & 1 & & & \\
\hline $\mathrm{RH}$ & 0.07 & 0.00 & -0.01 & $-0.27 * *$ & $0.15 * *$ & 0.06 & $0.11 * *$ & -0.05 & 1 & & \\
\hline AirVelocity & $-0.08^{*}$ & $0.14^{* *}$ & -0.04 & $-0.15^{* *}$ & $0.07 *$ & $0.17^{* *}$ & -0.04 & $0.16^{* *}$ & $0.20 * *$ & 1 & \\
\hline RadiantTemp & $-0.10 * *$ & $0.11 * *$ & $-0.21 * *$ & $-0.23 * *$ & 0.03 & $0.09 *$ & $0.12 * *$ & $0.96 * *$ & -0.05 & $0.11 * *$ & 1 \\
\hline
\end{tabular}

**. Correlation is significant at the 0.01 level (2-tailed).

*. Correlation is significant at the 0.05 level (2-tailed). 
B-2: Matched GPOE + NCR Dataset

\begin{tabular}{|c|c|c|c|c|c|c|c|c|c|c|c|}
\hline & Age & Sex & Window & $\begin{array}{c}\text { WSEnclo } \\
\text { sure }\end{array}$ & $\begin{array}{l}\text { AveIlluD } \\
\text { esk }\end{array}$ & AW & SII & AirTemp & RH & $\begin{array}{c}\text { AirVeloci } \\
\text { ty }\end{array}$ & $\begin{array}{c}\text { RadiantT } \\
\text { emp }\end{array}$ \\
\hline Age & 1 & & & & & & & & & & \\
\hline Sex & 0.09 & 1 & & & & & & & & & \\
\hline Window & 0.14 & 0.03 & 1 & & & & & & & & \\
\hline WSEnclosure & 0.12 & 0.07 & 0.10 & 1 & & & & & & & \\
\hline AveIlluDesk & 0.01 & -0.01 & $0.31 * *$ & -0.03 & 1 & & & & & & \\
\hline AW & -0.08 & -0.10 & 0.01 & $-0.19 * *$ & -0.02 & 1 & & & & & \\
\hline SII & -0.03 & -0.09 & 0.00 & $-0.49 * *$ & 0.00 & $-0.27 * *$ & 1 & & & & \\
\hline AirTemp & -0.10 & 0.07 & -0.06 & -0.04 & $0.28 * *$ & 0.02 & $-0.16^{*}$ & 1 & & & \\
\hline $\mathrm{RH}$ & -0.06 & -0.03 & 0.02 & 0.12 & -0.12 & $0.15 *$ & 0.00 & -0.10 & 1 & & \\
\hline AirVelocity & 0.08 & -0.04 & 0.09 & -0.04 & 0.07 & $0.26^{* *}$ & -0.01 & -0.01 & 0.09 & 1 & \\
\hline RadiantTemp & -0.09 & 0.04 & -0.11 & -0.10 & $0.32 * *$ & 0.04 & -0.13 & $0.95 * *$ & -0.07 & 0.01 & 1 \\
\hline
\end{tabular}

* Correlation is significant at the 0.05 level (2-tailed). 


\section{B-3: Survey-Only NCR Dataset}

\begin{tabular}{|c|c|c|c|c|c|c|c|c|c|c|c|c|c|c|c|c|c|c|}
\hline & Age & Sex & $\begin{array}{c}\text { Windo } \\
\mathrm{w} \\
\text { Locatio } \\
\mathrm{n}\end{array}$ & $\begin{array}{c}\text { Workst } \\
\text { ation } \\
\text { Enclos } \\
\text { ure } \\
\end{array}$ & $\begin{array}{c}\text { Unassi } \\
\text { gned } \\
\text { WS }\end{array}$ & SAT_L & $\begin{array}{c}\text { SAT_A } \\
\text { P }\end{array}$ & $\begin{array}{c}\text { SAT_V } \\
\mathrm{T}^{-}\end{array}$ & $\begin{array}{c}\text { TRAN } \\
\text { S_AA }\end{array}$ & $\begin{array}{l}\text { TRAN } \\
\text { S_PR } \\
\end{array}$ & $\begin{array}{l}\text { TRAN } \\
\text { S_PU } \\
\end{array}$ & $\begin{array}{c}\text { ONSIT } \\
\text { E }\end{array}$ & $\begin{array}{c}\text { OUTD } \\
\text { OOR } \\
\end{array}$ & $\begin{array}{c}\text { SUSTA } \\
\text { IN }\end{array}$ & $\begin{array}{c}\text { IN_OF } \\
\text { FICE }\end{array}$ & FOOD & $\begin{array}{c}\text { ACCE } \\
\text { SS }\end{array}$ & $\begin{array}{c}\text { HEAL } \\
\text { TH }\end{array}$ \\
\hline Age & 1 & & & & & & & & & & & & & & & & & \\
\hline Sex & $0.08^{* *}$ & 1 & & & & & & & & & & & & & & & & \\
\hline $\begin{array}{l}\text { Window } \\
\text { Location }\end{array}$ & $0.12^{* *}$ & -0.03 & 1 & & & & & & & & & & & & & & & \\
\hline $\begin{array}{l}\text { WS } \\
\text { Enclosure }\end{array}$ & 0.04 & $-0.06^{*}$ & 0.03 & 1 & & & & & & & & & & & & & & \\
\hline $\begin{array}{l}\text { Unassigned } \\
\text { WS }\end{array}$ & $-0.08^{* *}$ & 0.0 & 0.05 & $-0.32^{* *}$ & 1 & & & & & & & & & & & & & \\
\hline SAT_L & $0.06^{* *}$ & $0.06^{* *}$ & $0.40^{* *}$ & $0.05^{*}$ & 0.02 & 1 & & & & & & & & & & & & \\
\hline SAT_AP & -0.02 & -0.02 & $0.17^{* *}$ & $0.23^{* *}$ & $-0.05^{*}$ & $0.62^{* *}$ & 1 & & & & & & & & & & & \\
\hline SAT_VT & 0.03 & $0.10^{* *}$ & $0.08^{* *}$ & 0.00 & $0.12^{* *}$ & $0.60^{* *}$ & $0.54^{* *}$ & 1 & & & & & & & & & & \\
\hline TRANS_A & -0.02 & -0.05 & 0.00 & -0.03 & 0.03 & $0.17^{* *}$ & $0.16^{* *}$ & $0.17^{* *}$ & 1 & & & & & & & & & \\
\hline TRANS_PR & $0.18^{* *}$ & -0.01 & 0.04 & -0.02 & 0.03 & $0.20^{* *}$ & $0.26^{* *}$ & $0.23^{* *}$ & $0.31^{* *}$ & 1 & & & & & & & & \\
\hline TRANS_PU & 0.05 & 0.02 & 0.01 & -0.01 & 0.01 & $0.22^{* *}$ & $0.20^{* *}$ & $0.21^{* *}$ & $0.22^{* *}$ & $0.20^{* *}$ & 1 & & & & & & & \\
\hline ONSITE & $0.10^{* *}$ & -0.01 & 0.02 & 0.03 & -0.04 & $0.37^{* *}$ & $0.40^{* *}$ & $0.38^{* *}$ & $0.30^{* *}$ & $0.28^{* *}$ & $0.32^{* * *}$ & 1 & & & & & & \\
\hline OUTDOOR & 0.05 & 0.03 & 0.03 & 0.00 & -0.04 & $0.31^{* *}$ & $0.32^{* *}$ & $0.31^{* *}$ & $0.31^{* *}$ & $0.26^{* *}$ & $0.30^{* *}$ & $0.65^{* *}$ & 1 & & & & & \\
\hline SUSTAIN & $0.09^{* *}$ & -0.03 & 0.01 & -0.01 & $-0.06^{*}$ & $0.41^{* *}$ & $0.44^{* *}$ & $0.41^{* *}$ & $0.25^{* *}$ & $0.30^{* *}$ & $0.30^{* *}$ & $0.52^{* *}$ & $0.46^{* *}$ & 1 & & & & \\
\hline IN_OFFICE & 0.00 & -0.02 & $0.06^{* *}$ & $0.06^{*}$ & -0.02 & $0.49^{* *}$ & $0.62^{* *}$ & $0.49^{* *}$ & $0.21^{* *}$ & $0.31^{* *}$ & $0.30^{* *}$ & $0.51^{* *}$ & $0.45^{* *}$ & $0.61^{* *}$ & 1 & & & \\
\hline FOOD & 0.02 & 0.02 & -0.02 & 0.00 & $0.07^{* *}$ & $0.35^{* *}$ & $0.42^{* *}$ & $0.40^{* *}$ & $0.23^{* *}$ & $0.24^{* *}$ & $0.24^{* *}$ & $0.52^{* *}$ & $0.48^{* *}$ & $0.50^{* *}$ & $0.60^{* *}$ & 1 & & \\
\hline ACCESS & 0.00 & 0.00 & $0.08^{* *}$ & 0.03 & $-0.09^{* *}$ & $0.48^{* *}$ & $0.53^{* *}$ & $0.42^{* *}$ & $0.25^{* *}$ & $0.26^{* *}$ & $0.36^{* *}$ & $0.49^{* *}$ & $0.49^{* *}$ & $0.58^{* *}$ & $0.66^{* *}$ & $0.52^{* *}$ & 1 & \\
\hline HEALTH & 0.02 & 0.01 & 0.04 & 0.00 & -0.01 & $0.46^{* *}$ & $0.49^{* *}$ & $0.50^{* *}$ & $0.37^{* *}$ & $0.32^{* *}$ & $0.40^{* *}$ & $0.69^{* *}$ & $0.66^{* *}$ & $0.68^{* *}$ & $0.65^{* *}$ & $0.58^{* *}$ & $0.61^{* *}$ & 1 \\
\hline
\end{tabular}

**. Correlation is significant at the 0.01 level (2-tailed).

*. Correlation is significant at the 0.05 level (2-tailed). 


\section{APPENDIX C - Principal Components Analysis Table}

\begin{tabular}{|c|c|c|c|c|c|c|c|c|c|c|}
\hline \multirow[b]{2}{*}{ Individual questionnaire items (satisfaction with) } & \multicolumn{10}{|c|}{ Component } \\
\hline & 1 & 2 & 3 & 4 & 5 & 6 & 7 & 8 & 9 & 10 \\
\hline Supports for your transportation choices: showers & 0.10 & 0.07 & 0.01 & 0.05 & 0.03 & 0.73 & 0.09 & 0.09 & 0.29 & 0.07 \\
\hline $\begin{array}{l}\text { Supports for your transportation choices: } \\
\text { storage/locker for personal use }\end{array}$ & 0.01 & 0.17 & 0.09 & 0.10 & 0.13 & 0.55 & 0.17 & -0.03 & 0.38 & 0.00 \\
\hline $\begin{array}{l}\text { Supports for your transportation choices: } \\
\text { storage/locker for bicycles (or other active } \\
\text { transportation equipment) }\end{array}$ & 0.12 & 0.11 & -0.07 & 0.09 & 0.07 & 0.64 & 0.11 & 0.00 & 0.18 & -0.04 \\
\hline $\begin{array}{l}\text { Supports for your transportation choices: } \\
\text { maintenance/repair station for bicycles (or other active } \\
\text { transportation equipment) }\end{array}$ & 0.04 & -0.03 & 0.08 & 0.08 & 0.11 & 0.17 & 0.03 & 0.03 & 0.66 & -0.07 \\
\hline $\begin{array}{l}\text { Supports for your transportation choices: electric } \\
\text { vehicle charging stations }\end{array}$ & 0.08 & -0.01 & 0.07 & -0.12 & 0.02 & 0.14 & -0.05 & 0.07 & 0.67 & 0.16 \\
\hline $\begin{array}{l}\text { Supports for your transportation choices: ways to find } \\
\text { people with whom to share transportation options }\end{array}$ & 0.18 & 0.04 & 0.05 & -0.08 & 0.11 & 0.18 & 0.07 & 0.01 & 0.52 & 0.33 \\
\hline $\begin{array}{l}\text { Supports for your transportation choices: availability } \\
\text { of parking }\end{array}$ & 0.07 & 0.15 & 0.10 & 0.24 & 0.01 & 0.06 & 0.00 & -0.02 & 0.10 & 0.75 \\
\hline $\begin{array}{l}\text { Supports for your transportation choices: cost of } \\
\text { parking }\end{array}$ & 0.01 & 0.13 & 0.02 & 0.06 & 0.03 & -0.01 & 0.11 & 0.07 & 0.11 & 0.82 \\
\hline $\begin{array}{l}\text { Supports for your transportation choices: location of } \\
\text { public transit stops near your work destination }\end{array}$ & -0.02 & 0.02 & 0.09 & 0.10 & 0.02 & 0.12 & 0.78 & 0.09 & -0.06 & 0.04 \\
\hline $\begin{array}{l}\text { Supports for your transportation choices: information } \\
\text { about status of public transit }\end{array}$ & 0.26 & 0.17 & 0.01 & 0.10 & 0.15 & 0.09 & 0.73 & 0.00 & 0.09 & 0.09 \\
\hline $\begin{array}{l}\text { Supports for your transportation choices: availability } \\
\text { of public transit at your preferred time }\end{array}$ & 0.14 & 0.12 & 0.07 & 0.12 & 0.03 & 0.09 & 0.81 & 0.08 & 0.06 & 0.03 \\
\hline On-site amenities and their features: daycare & 0.08 & 0.08 & 0.12 & -0.25 & 0.48 & 0.01 & 0.14 & 0.15 & 0.05 & 0.21 \\
\hline On-site amenities and their features: shopping & 0.28 & 0.17 & 0.11 & 0.11 & 0.65 & 0.09 & 0.08 & 0.01 & 0.01 & -0.04 \\
\hline On-site amenities and their features: banking & 0.13 & 0.08 & 0.05 & 0.21 & 0.57 & 0.03 & -0.05 & 0.07 & 0.14 & -0.09 \\
\hline $\begin{array}{l}\text { On-site amenities and their features: places to } \\
\text { socialize }\end{array}$ & 0.54 & 0.11 & 0.32 & 0.27 & 0.32 & 0.07 & 0.08 & -0.05 & 0.07 & -0.12 \\
\hline $\begin{array}{l}\text { On-site amenities and their features: places to be calm } \\
\text { and contemplative }\end{array}$ & 0.62 & 0.09 & 0.26 & 0.18 & 0.20 & 0.04 & 0.16 & -0.05 & 0.22 & 0.03 \\
\hline $\begin{array}{l}\text { On-site amenities and their features: artwork in public } \\
\text { spaces }\end{array}$ & 0.70 & 0.16 & 0.12 & 0.03 & 0.08 & -0.05 & 0.04 & 0.03 & 0.26 & -0.02 \\
\hline $\begin{array}{l}\text { On-site amenities and their features: aesthetic } \\
\text { appearance of amenity spaces }\end{array}$ & 0.69 & 0.22 & 0.25 & 0.16 & 0.17 & 0.02 & 0.08 & -0.02 & 0.14 & 0.04 \\
\hline Places to eat and socialize with colleagues & 0.34 & 0.22 & 0.69 & 0.17 & 0.13 & 0.08 & 0.04 & 0.04 & 0.07 & 0.02 \\
\hline Facilities to store and prepare food & 0.19 & 0.30 & 0.76 & 0.10 & 0.12 & 0.07 & 0.02 & 0.11 & 0.10 & -0.02 \\
\hline Facilities to wash and store reusable dishes/utensils & 0.23 & 0.28 & 0.73 & 0.14 & 0.11 & 0.09 & 0.06 & 0.15 & 0.12 & -0.02 \\
\hline $\begin{array}{l}\text { On-site amenities related to the nearby exterior } \\
\text { environment: outdoor seating }\end{array}$ & 0.61 & -0.01 & 0.27 & 0.08 & 0.22 & 0.09 & 0.02 & 0.19 & -0.03 & 0.09 \\
\hline $\begin{array}{l}\text { On-site amenities related to the nearby exterior } \\
\text { environment: drinking fountains }\end{array}$ & 0.32 & -0.02 & 0.26 & 0.07 & 0.05 & 0.01 & 0.00 & 0.62 & 0.12 & 0.14 \\
\hline $\begin{array}{l}\text { On-site amenities related to the nearby exterior } \\
\text { environment: pedestrian paths }\end{array}$ & 0.56 & 0.13 & 0.02 & 0.04 & 0.06 & 0.39 & -0.01 & 0.37 & -0.23 & 0.08 \\
\hline $\begin{array}{l}\text { On-site amenities related to the nearby exterior } \\
\text { environment: green space }\end{array}$ & 0.68 & 0.12 & 0.04 & 0.01 & 0.11 & 0.26 & 0.04 & 0.28 & -0.17 & 0.07 \\
\hline $\begin{array}{l}\text { On-site amenities related to the nearby exterior } \\
\text { environment: gathering places }\end{array}$ & 0.65 & 0.13 & 0.23 & 0.21 & 0.22 & 0.25 & 0.06 & 0.11 & -0.08 & -0.01 \\
\hline $\begin{array}{l}\text { Availability of programs and design features that } \\
\text { support a more sustainable working environment }\end{array}$ & 0.40 & 0.35 & -0.09 & 0.20 & 0.30 & -0.11 & 0.14 & 0.29 & 0.32 & 0.12 \\
\hline Usability of recycling and composting centres & 0.11 & 0.69 & 0.12 & 0.14 & 0.18 & 0.08 & -0.03 & 0.11 & -0.10 & 0.09 \\
\hline Distance to waste collection point & 0.08 & 0.65 & 0.15 & 0.23 & 0.14 & 0.09 & -0.03 & 0.22 & -0.12 & 0.08 \\
\hline Support for reducing paper use & 0.18 & 0.64 & 0.10 & 0.08 & 0.05 & 0.09 & 0.03 & 0.08 & 0.13 & 0.10 \\
\hline $\begin{array}{l}\text { On-site amenities and their features: sustainability } \\
\text { information and displays }\end{array}$ & 0.37 & 0.25 & 0.01 & 0.07 & 0.47 & -0.13 & 0.04 & 0.06 & 0.23 & 0.12 \\
\hline
\end{tabular}




\section{APPENDIX C - Principal Components Analysis Table (cont'd)}

\begin{tabular}{|c|c|c|c|c|c|c|c|c|c|c|}
\hline \multirow[b]{2}{*}{ Individual questionnaire items (satisfaction with) } & \multicolumn{10}{|c|}{ Component } \\
\hline & 1 & 2 & 3 & 4 & 5 & 6 & 7 & 8 & 9 & 10 \\
\hline IT connectivity and tools to support work & 0.21 & 0.55 & 0.22 & 0.12 & 0.11 & 0.06 & 0.25 & 0.08 & 0.08 & -0.03 \\
\hline $\begin{array}{l}\text { Access to office supplies, materials, and printing } \\
\text { facilities }\end{array}$ & 0.03 & 0.50 & 0.23 & 0.15 & 0.05 & 0.09 & 0.14 & 0.09 & 0.02 & 0.12 \\
\hline Availability of small meeting rooms & 0.24 & 0.19 & 0.53 & 0.21 & 0.11 & -0.17 & 0.10 & 0.16 & 0.02 & 0.16 \\
\hline Availability of large meeting rooms & 0.22 & 0.23 & 0.54 & 0.20 & 0.06 & -0.14 & 0.06 & 0.21 & 0.00 & 0.17 \\
\hline Availability of preferred work locations & 0.29 & 0.43 & 0.31 & 0.24 & 0.04 & -0.04 & 0.10 & -0.19 & 0.10 & 0.20 \\
\hline Spaciousness of your workspace surroundings & 0.07 & 0.25 & 0.08 & 0.67 & 0.02 & 0.07 & 0.03 & 0.00 & -0.04 & 0.05 \\
\hline Speed and availability of elevators & 0.16 & 0.19 & 0.13 & 0.42 & 0.08 & 0.24 & 0.15 & 0.20 & -0.07 & 0.15 \\
\hline Access points from the building to the outside & 0.19 & 0.17 & 0.11 & 0.69 & 0.15 & -0.01 & 0.13 & 0.23 & 0.02 & 0.06 \\
\hline Ability to find your way around inside the building & 0.16 & 0.09 & 0.19 & 0.65 & 0.13 & 0.01 & 0.11 & 0.15 & 0.04 & 0.14 \\
\hline Ability to locate co-workers when needed & 0.15 & 0.33 & 0.26 & 0.45 & 0.04 & 0.08 & 0.12 & -0.10 & 0.00 & 0.03 \\
\hline Access to stairs to move between floors & 0.12 & 0.33 & 0.32 & 0.41 & 0.02 & 0.16 & 0.06 & 0.20 & -0.03 & 0.00 \\
\hline $\begin{array}{l}\text { Availability of programs and design features that } \\
\text { support a more healthful working environment }\end{array}$ & 0.36 & 0.32 & -0.04 & 0.22 & 0.31 & -0.08 & 0.18 & 0.30 & 0.29 & 0.06 \\
\hline Access to water fountain/bottle refill stations & 0.03 & 0.32 & 0.28 & 0.19 & 0.08 & 0.08 & 0.06 & 0.64 & -0.03 & -0.04 \\
\hline $\begin{array}{l}\text { Quality of water from water fountain/bottle refill } \\
\text { stations }\end{array}$ & 0.05 & 0.26 & 0.05 & 0.13 & 0.06 & 0.07 & 0.14 & 0.65 & 0.09 & -0.02 \\
\hline Cleanliness and maintenance of public spaces & 0.21 & 0.61 & 0.18 & 0.10 & 0.11 & 0.07 & 0.10 & 0.10 & -0.01 & 0.01 \\
\hline $\begin{array}{l}\text { Natural materials and elements (real or simulated) in } \\
\text { the workplace }\end{array}$ & 0.47 & 0.33 & 0.26 & 0.15 & 0.12 & 0.02 & 0.08 & 0.02 & 0.08 & 0.03 \\
\hline $\begin{array}{l}\text { On-site amenities and their features: view of nature in } \\
\text { amenity spaces }\end{array}$ & 0.78 & 0.19 & 0.10 & 0.07 & 0.07 & 0.06 & 0.12 & 0.02 & 0.11 & 0.06 \\
\hline On-site amenities and their features: fitness & 0.20 & -0.06 & 0.34 & -0.10 & 0.51 & 0.30 & 0.10 & 0.07 & -0.09 & 0.29 \\
\hline $\begin{array}{l}\text { On-site amenities and their features: healthy food } \\
\text { options }\end{array}$ & 0.20 & 0.21 & 0.11 & 0.20 & 0.60 & 0.19 & 0.06 & -0.04 & 0.10 & -0.03 \\
\hline $\begin{array}{l}\text { On-site amenities related to the nearby exterior } \\
\text { environment: exercise opportunities }\end{array}$ & 0.43 & 0.09 & 0.14 & 0.04 & 0.35 & 0.49 & 0.02 & 0.15 & -0.21 & 0.19 \\
\hline
\end{tabular}

Extraction Method: Principal Component Analysis. Rotation Method: Varimax with Kaiser Normalization. 


\section{References}

Agha-Hossein, M. M., El-Jouzi, S., Elmualim, A. A., Ellis, J., \& Williams, M. (2013). Postoccupancy studies of an office environment: Energy performance and occupants' satisfaction. Building and Environment, 69, 121-130. https://doi.org/10.1016/j.buildenv.2013.08.003

Al Horr, Y., Arif, M., Kaushik, A., Mazroei, A., Elsarrag, E., \& Mishra, S. (2017). Occupant productivity and indoor environment quality: A case of GSAS. International Journal of Sustainable Built Environment, 6(2), 476-490. https://doi.org/10.1016/j.ijsbe.2017.11.001

Ali, A. S., Chua, S. J. L., \& Lim, M. E.-L. (2015). The effect of physical environment comfort on employees performance in office buildings: A case study of three public universities in Malaysia. Structural Survey, 33(4/5), 294-308. https://doi.org/10.1108/SS-02-2015-0012

Altomonte, S., Schiavon, S., Kent, M. G., \& Brager, G. (2017). Indoor environmental quality and occupant satisfaction in green-certified buildings. Building Research and Information, 47(3), 255-274. https://doi.org/10.1080/09613218.2018.1383715

Apte, M. G., Fisk, W. J., \& Daisey, J. M. (2000). Associations Between Indoor CO2 Concentrations and Sick Building Syndrome Symptoms in U.S. Office Buildings: An Analysis of the 1994-1996 BASE Study Data. Indoor Air, 10(4), 246-257. https://doi.org/10.1034/j.1600-0668.2000.010004246.x

Aries, M. B. C., Veitch, J. A., \& Newsham, G. R. (2010). Windows, view, and office characteristics predict physical and psychological discomfort. Journal of Environmental Psychology, 30(4), 533-541. https://doi.org/10.1016/j.jenvp.2009.12.004

Barton, L. (1992). Corporate sponsored child care: A benefit with high satisfaction, questionable future. International Journal of Manpower, 13(1), 12-24. https://doi.org/10.1108/EUM0000000000899

Bergs, J. (2002). The effect of healthy workplaces on the well-being and productivity of office workers. International Plants for People Symposium, 1-12. Amsterdam, NL.

Bernstein, E. S., \& Turban, S. (2018). The impact of the 'open' workspace on human collaboration. Philosophical Transactions of the Royal Society B: Biological Sciences, 373(1753), 20170239. https://doi.org/10.1098/rstb.2017.0239

Bluyssen, P. M., Aries, M., \& van Dommelen, P. (2011). Comfort of workers in office buildings: The European HOPE project. Building and Environment, 46(1), 280-288. https://doi.org/10.1016/j.buildenv.2010.07.024

Bradley, J.S., \& Gover, B. N. (2004). Criteria for acoustic comfort in open-plan offices. Proceedings of the 33rd International Congress and Exposition on Noise Control Engineering, 1-6. Prague, Czech Republic.

Bradley, John S. (2003). The Acoustical design of conventional open plan offices. Canadian Acoustics, 27(3), 23-31. https://doi.org/NRCC-46274 A

Brill, M., Weidemann, S., \& BOSTI. (2001). Disproving Widespread Myths about Workplace Design. https://doi.org/10.1108/f.2003.06921aae.003 
Brookes, M. J., \& Kaplan, A. (1972). The office environment: space planning and effective behavior. Human Factors, 14(5), 373-391.

Brown, Z., Cole, R. J., Robinson, J., \& Dowlatabadi, H. (2010). Evaluating user experience in green buildings in relation to workplace culture and context. Facilities, 28(3/4), 225-238. https://doi.org/10.1108/02632771011023168

Carey, D., Bortoluzzi, B., \& McArthur, J. J. (n.d.). A Multi-Dimensional Approach to Evaluating Productivity within the Knowledge Worker Context. Transdisciplinary Workplace Research Conference.

Charles, K. E., Veitch, J. A., Newsham, G. R., Marquardt, C. J. G., \& Geerts, J. (2006). Satisfaction with ventilation in open-plan offices : COPE field findings. Proceedings of Healthy Buildings 2006: Creating a Healthy Indoor Environment for People, 93-98. Lisboa, Portugal: International Society of Indoor Air Quality and Climate.

Cheung, T., Schiavon, S., Parkinson, T., Li, P., \& Brager, G. (2019). Analysis of the accuracy on PMV - PPD model using the ASHRAE Global Thermal Comfort Database II. Building and Environment, 153, 205-217. https://doi.org/10.1016/j.buildenv.2019.01.055

Choi, J. H., Aziz, A., \& Loftness, V. (2010). Investigation on the impacts of different genders and ages on satisfaction with thermal environments in office buildings. Building and Environment, 45(6), 1529-1535. https://doi.org/10.1016/j.buildenv.2010.01.004

Choi, J. H., Loftness, V., \& Aziz, A. (2012). Post-occupancy evaluation of 20 office buildings as basis for future IEQ standards and guidelines. Energy and Buildings, 46, 167-175. https://doi.org/10.1016/j.enbuild.2011.08.009

Cohen, J. (1988). Statistical Power Analysis for the Behavioral Sciences (2nd ed.). Hillsdale, NJ: Erlbaum.

Colarelli, S. M. (1984). Methods of communication and mediating processes in realistic job previews. Journal of Applied Psychology, 69(4), 633-642. https://doi.org/10.1037/00219010.69.4.633

De Croon, E. M., Sluiter, J. K., Kuijer, P. P. F. M., \& Frings-Dresen, M. H. W. (2005). The effect of office concepts on worker health and performance: A systematic review of the literature. Ergonomics, 48(2), 119-134. https://doi.org/10.1080/00140130512331319409

Dolbier, C. L., Webster, J. A., McCalister, K. T., Mallon, M. W., \& Steinhardt, M. A. (2005). Reliability and validity of a single-item measure of job satisfaction. American Journal of Health Promotion, 19, 194-198. https://doi.org/10.4278/0890-1171-19.3.194

Elzeyadi, I. (2011). Daylighting-Bias and Biophilia: Quantifying the Impact of Daylighting on Occupants Health. Retrieved from US Green Building Council website: https://www.usgbc.org/resources/daylighting-bias-and-biophilia-quantifying-impactdaylighting-occupants-health

Ettema, D., Gärling, T., Eriksson, L., Friman, M., Olsson, L. E., \& Fujii, S. (2011). Satisfaction with travel and subjective well-being: Development and test of a measurement tool. Transportation Research Part F: Traffic Psychology and Behaviour, 14(3), 167-175. https://doi.org/10.1016/j.trf.2010.11.002

Farley, K. M. J., \& Veitch, J. A. (2001). A room with a view: A review of the effects of windows 
on work and well-being. In Research Report RR-136. Retrieved from https://nparc.nrccnrc.gc.ca/eng/view/fulltext/?id=ca18fccf-3ac9-4190-92d9-dc6cbbca7a98

Fisk, W. J., Black, D., \& Brunner, G. (2011). Benefits and costs of improved IEQ in U.S. offices. Indoor Air, 21(5), 357-367. https://doi.org/10.1111/j.1600-0668.2011.00719.x

Foju, B. A. (1993). The role of facilities management (maintenance) on office worker performance in Yaoundé, Cameroon. University of Michigan.

Frontczak, M., Schiavon, S., Goins, J., Arens, E., Zhang, H., \& Wargocki, P. (2012). Quantitative relationships between occupant satisfaction and satisfaction aspects of indoor environmental quality and building design. Indoor Air, 22(2), 119-131. https://doi.org/10.1111/j.1600-0668.2011.00745.x

Galasiu, A. D., \& Veitch, J. A. (2006). Occupant preferences and satisfaction with the luminous environment and control systems in daylit offices: a literature review. Energy and Buildings, 38(7), 728-742. https://doi.org/10.1016/j.enbuild.2006.03.001

Gensler. (2019). U.S. Workplace Survey 2019. Retrieved from https://www.gensler.com/research-insight/workplace-surveys/us/2019

Getka, K. (2012). Amenities provided as predictors of job satisfaction among entry-level, liveon/live-in housing and residence life profesionals. University of Central Florida.

Gray, T., \& Birrell, C. (2014). Are biophilic-designed site office buildings linked to health benefits and high performing occupants? International Journal of Environmental Research and Public Health, 11(12), 12204-12222. https://doi.org/10.3390/ijerph111212204

Hair, J. F., Black, W. C., Bajin, B. J., \& Anderson, R. E. (2014). Multivariate data analysis (7th ed.). Essex, UK: Pearson.

Hedge, A., Erickson, W. A., \& Rubin, G. (1992). Effects of personal and occupational factors on sick building syndrome reports in air-conditioned offices. In Stress \& well-being at work: Assessments and interventions for occupational mental health. (pp. 286-298). https://doi.org/10.1037/10116-019

Holmgren, M., Kabanshi, A., \& Sörqvist, P. (2017). Occupant perception of "green" buildings: Distinguishing physical and psychological factors. Building and Environment, 114, 140147. https://doi.org/10.1016/j.buildenv.2016.12.017

Hotelling, H. (1933). Analysis of a complex of statistical variables into principal components. Journal of Educational Psychology, 24(6), 417-441. https://doi.org/10.1037/h0071325

Hua, Y., Loftness, V., Heerwagen, J. H., \& Powell, K. M. (2011). Relationship between workplace spatial settings and occupant-perceived support for collaboration. Environment and Behavior, 43(6), 807-826. https://doi.org/10.1177/0013916510364465

Huizenga, C., Abbaszadeh, S., Zagreus, L., \& Arens, E. (2006). Air quality and thermal comfort in office buildings: Results of a large indoor environmental quality survey. Proceeding of Healthy Buildings - Center for the Built Environment, University of California, 3, 393-397.

Hwang, T., \& Kim, J. T. (2011). Effects of indoor lighting on occupants' visual comfort and eye health in a green building. Indoor and Built Environment, 20(1), 75-90. https://doi.org/10.1177/1420326X10392017 
International WELL Building Institute. (2014). The WELL Building Standard Version 1.0. Retrieved from https://www.wellcertified.com/en/standard

Kamarulzaman, N., Saleh, A. A., Hashim, S. Z., Hashim, H., \& Abdul-Ghani, A. A. (2011). An overview of the influence of physical office environments towards employees. Procedia Engineering, 20, 262-268. https://doi.org/10.1016/j.proeng.2011.11.164

Kano, N., Seraku, N., Takahashi, F., \& Tsuji, S. (1984). Attractive quality and must-be quality. Journal of the Japanese Society for Quality Control, 41, 39-48.

Karjalainen, S. (2007). Gender differences in thermal comfort and use of thermostats in everyday thermal environments. Building and Environment, 42(4), 1594-1603. https://doi.org/10.1016/j.buildenv.2006.01.009

Kerlinger, F. N., \& Lee, H. B. (2000). Foundations of Behavioral Research (4th ed.). Fort Worth, TX: Harcourt College Publishers.

Kim, J., \& de Dear, R. (2012). Nonlinear relationships between individual IEQ factors and overall workspace satisfaction. Building and Environment, 49(1), 33-40. https://doi.org/10.1016/j.buildenv.2011.09.022

Kim, J., \& de Dear, R. (2013). Workspace satisfaction: The privacy-communication trade-off in open-plan offices. Journal of Environmental Psychology, 36, 18-26.

https://doi.org/10.1016/j.jenvp.2013.06.007

Kim, J., de Dear, R., Cândido, C., Zhang, H., \& Arens, E. (2013). Gender differences in office occupant perception of indoor environmental quality (IEQ). Building and Environment, 70, 245-256. https://doi.org/10.1016/j.buildenv.2013.08.022

Klepeis, N. E., Nelson, W. C., Ott, W. R., Robinson, J. P., Tsang, A. M., Switzer, P., ... Engelmann, W. H. (2001). The National Human Activity Pattern Survey (NHAPS): A resource for assessing exposure to environmental pollutants. Journal of Exposure Analysis and Environmental Epidemiology, 11(3), 231-252. https://doi.org/10.1038/sj.jea.7500165

Kline, R. B. (1997). Principles and Practice of Structural Equation Modeling. New York: Guilford Press.

Klitzman, S., \& Stellman, J. M. (1989). The impact of the physical environment on the psychological well-being of office workers. Social Science \& Medicine, 29(6), 733-742. https://doi.org/10.1016/0277-9536(89)90153-6

Kwon, S. H., Chun, C., \& Kwak, R. Y. (2011). Relationship between quality of building maintenance management services for indoor environmental quality and occupant satisfaction. Building and Environment, 46(11), 2179-2185. https://doi.org/10.1016/j.buildenv.2011.04.028

Laing, A. (2005). DEGW approaches to workplace performance measurement. Presentation to Work Environments Network at EDRA 35 (Environmental Design Research Association). Vancouver, BC.

Lakeridou, M., Ucci, M., Marmot, A., \& Ridley, I. (2012). The potential of increasing cooling set-points in air-conditioned offices in the UK. Applied Energy, 94, 338-348.

https://doi.org/10.1016/j.apenergy.2012.01.064 
Leaman, A. (1995). Dissatisfaction and Office Productivity. Facilities, 13(2), 13-19. https://doi.org/10.1108/02632779510078120

Leaman, A., Stevenson, F., \& Bordass, B. (2010). Building evaluation: Practice and principles. Building Research and Information, 38(5), 564-577.

https://doi.org/10.1080/09613218.2010.495217

Leder, S., Newsham, G. R., Veitch, J. A., Mancini, S., \& Charles, K. E. (2016). Effects of office environment on employee satisfaction: A new analysis. Building Research and Information, 44(1), 34-50. https://doi.org/10.1080/09613218.2014.1003176

Lee, J. Y., Wargocki, P., Chan, Y. H., Chen, L., \& Tham, K. W. (2019). Indoor environmental quality, occupant satisfaction, and acute building-related health symptoms in Green Markcertified compared with non-certified office buildings. Indoor Air, 29(1), 112-129. https://doi.org/10.1111/ina.12515

Lee, S. Y., \& Kim, J. H. (2014). Effects of servicescape on perceived service quality, satisfaction and behavioral outcomes in public service facilities. Journal of Asian Architecture and Building Engineering, 13(1), 125-131. https://doi.org/10.3130/jaabe.13.125

Lee, Y. S. (2010). Office layout affecting privacy, interaction, and acoustic quality in LEEDcertified buildings. Building and Environment, 45(7), 1594-1600. https://doi.org/10.1016/j.buildenv.2010.01.007

Leech, J. A., Nelson, W. C., Burnett, R. T., \& Raizenne, M. E. (2002). It's about time: A comparison of Canadian and American time-activity patterns. Journal of Exposure Analysis and Environmental Epidemiology, 12, 427-432. https://doi.org/10.1038/sj.jea.7500244

Liang, H. H., Chen, C. P., Hwang, R. L., Shih, W. M., Lo, S. C., \& Liao, H. Y. (2014). Satisfaction of occupants toward indoor environment quality of certified green office buildings in Taiwan. Building and Environment, 72, 232-242. https://doi.org/10.1016/j.buildenv.2013.11.007

MacKerron, G., \& Mourato, S. (2013). Happiness is greater in natural environments. Global Environmental Change, 23(5), 992-1000. https://doi.org/10.1016/j.gloenvcha.2013.03.010

MacNaughton, P., Satish, U., Laurent, J. G. C., Flanigan, S., Vallarino, J., Coull, B., ... Allen, J. G. (2017). The impact of working in a green certified building on cognitive function and health. Building and Environment, 114, 178-186. https://doi.org/10.1016/j.buildenv.2016.11.041

MacNaughton, P., Spengler, J., Vallarino, J., Santanam, S., Satish, U., \& Allen, J. (2016). Environmental perceptions and health before and after relocation to a green building. Building and Environment, 104, 138-144. https://doi.org/10.1016/j.buildenv.2016.05.011

Martellotta, F., Simone, A., Della Crociata, S., \& D’Alba, M. (2016). Global comfort and indoor environment quality attributes for workers of a hypermarket in Southern Italy. Building and Environment, 95, 355-364. https://doi.org/10.1016/j.buildenv.2015.09.029

Matzler, K., Fuchs, M., \& Schubert, A. K. (2004). Employee satisfaction: Does Kano's model apply? Total Quality Management and Business Excellence, 15(9-10), 1179-1198. https://doi.org/10.1080/1478336042000255569

McIntyre, D. A. (1978). Response to Atmospheric Humidity at Comfortable Air Temperature: A 
Comparison of Three Experiments. The Annals of Occupational Hygiene, 21(2), 177-190. https://doi.org/10.1093/annhyg/21.2.177

Merriam Webster Online. (n.d.). Amenity [Def.1]. Retrieved March 10, 2019, from https://www.merriam-webster.com/dictionary/amenity

Meyer, J. P., Allen, N. J., \& Smith, C. A. (1993). Commitment to organizations and occupations: Extension and test of a three-component conceptualization. Journal of Applied Psychology, 78(4), 538-551. https://doi.org/10.1037/0021-9010.78.4.538

Nematchoua, M. K., Ricciardi, P., Orosa, J. A., Asadi, S., \& Choudhary, R. (2018). Influence of indoor environmental quality on the self-estimated performance of office workers in the tropical wet and hot climate of Cameroon. Journal of Building Engineering, 21, 141-148. https://doi.org/10.1016/j.jobe.2018.10.007

Newsham, G.R., Birt, B. J., Arsenault, C. D., Thompson, A. J. L., Veitch, J. A., Mancini, S., ... Burns, G. J. (2012). Do green buildings outperform conventional buildings? Indoor environment and energy performance in North American offices. https://doi.org/https://doi.org/10.4224/20857897

Newsham, G.R., Brand, J., Donnelly, C., Veitch, J. A., Aries, M. B. C., \& Charles, K. E. (2009). Linking indoor environment conditions to job satisfaction: A field study. Building Research and Information, 37(2), 129-147. https://doi.org/10.1080/09613210802710298

Newsham, G.R., Veitch, J. A., \& Charles, K. E. (2008). Risk factors for dissatisfaction with the indoor environment in open-plan offices: An analysis of COPE field study data. Indoor Air, 18(4), 271-282. https://doi.org/10.1111/j.1600-0668.2008.00525.x

Newsham, Guy R., Birt, B. J., Arsenault, C., Thompson, A. J. L., Veitch, J. A., Mancini, S., ... Burns, G. J. (2013). Do 'green' buildings have better indoor environments? New evidence. Building Research \& Information, 41(4), 415-434. https://doi.org/10.1080/09613218.2013.789951

Newsham, Guy R., Veitch, J. A., Charles, K. E., Marquardt, C. J. G., Geerts, J., Bradley, J. S., ... Reardon, J. T. (2002). Environmental Satisfaction in Open-Plan Environments: 4. Relationships Between Physical Variables.

Newsham, Guy R., Veitch, J. A., Zhang, M. Q. (Nikki), Galasiu, A. D., Henderson, I. S., \& Thompson, A. J. L. (2017). Improving Organizational Productivity with Building Automation Systems. Retrieved from http://www.caba.org/documents/reports/CABAIOPBAS-2017-Report.pdf

Nunnally, J. C. (1978). Psychometric Theory (2nd ed.). New York: McGraw-Hill.

Otterbring, T., Pareigis, J., Wästlund, E., Makrygiannis, A., \& Lindström, A. (2018). The relationship between office type and job satisfaction: Testing a multiple mediation model through ease of interaction and well-being. Scandinavian Journal of Work, Environment and Health, 44(3), 330-334. https://doi.org/10.5271/sjweh.3707

Pedhazur, E. J. (1997). Multiple Regression in Behavioral Research: Explanation and Prediction (3rd ed.). Fort Worth, TX: Harcourt Brace.

Pei, Z., Lin, B., Liu, Y., \& Zhu, Y. (2015). Comparative study on the indoor environment quality of green office buildings in China with a long-term field measurement and investigation. 
Building and Environment, 84, 80-88. https://doi.org/10.1016/j.buildenv.2014.10.015

Pejtersen, J., Allermann, L., Kristensen, T. S., \& Poulsen, O. M. (2006). Indoor climate, psychosocial work environment and symptoms in open-plan offices. Indoor Air, 16(5), 392401. https://doi.org/10.1111/j.1600-0668.2006.00444.x

Preiser, W. F. E., \& Vischer, J. (2005). Assessing Building Performance. Oxford: ButterworthHeinemann.

Robertson, M. M., Huang, Y. H., O’Neill, M. J., \& Schleifer, L. M. (2008). Flexible workspace design and ergonomics training: Impacts on the psychosocial work environment, musculoskeletal health, and work effectiveness among knowledge workers. Applied Ergonomics, 39(4), 482-494. https://doi.org/10.1016/j.apergo.2008.02.022

Schaufeli, W. B., Bakker, A. B., \& Salanova, M. (2006). The measurement of work engagement with a short questionnaire: A cross-national study. Educational and Psychological Measurement, 66(4), 701-716. https://doi.org/10.1177/0013164405282471

Seppanen, O. A., Fisk, W. J., \& Mendell, M. J. (1999). Association of Ventilation Rates and $\mathrm{CO} 2$ Concentrations with Health and Other Responses in Commercial and Institutional Buildings. Indoor Air, 9(4), 226-252. https://doi.org/10.1111/j.1600-0668.1999.00003.x

Shephard, R. J. (1992). Twelve years experience of a fitness program for the salaried employees of a Toronto Life Assurance Company. American Journal of Health Promotion, 6(4), 292301. https://doi.org/10.4278/0890-1171-6.4.292

Silva, M. F., Maas, S., Souza, H. A. de, \& Gomes, A. P. (2017). Post-occupancy evaluation of residential buildings in Luxembourg with centralized and decentralized ventilation systems, focusing on indoor air quality (IAQ). Assessment by questionnaires and physical measurements. Energy and Buildings, 148, 119-127. https://doi.org/10.1016/j.enbuild.2017.04.049

Tabachnick, G. G., \& Fidell, L. S. (2001). Using Multivariate Statistics (4th ed.). Boston, MA: Allyn \& Bacon.

Tanabe, S. ichi, Haneda, M., \& Nishihara, N. (2015). Workplace productivity and individual thermal satisfaction. Building and Environment, 91, 42-50. https://doi.org/10.1016/j.buildenv.2015.02.032

Tham, K. W., Wargocki, P., \& Tan, Y. F. (2015). Indoor environmental quality, occupant perception, prevalence of sick building syndrome symptoms, and sick leave in a Green Mark Platinum-rated versus a non-Green Mark-rated building: A case study. Science and Technology for the Built Environment, 21(1), 35-44. https://doi.org/10.1080/10789669.2014.967164

Thayer, J. F., Verkuil, B., Brosschot, J. F., Kevin, K., West, A., Sterling, C., ... Sternberg, E. M. (2010). Effects of the physical work environment on physiological measures of stress. European Journal of Preventive Cardiology, 17(4), 431-439. https://doi.org/10.1097/HJR.0b013e328336923a

Veitch, J. A., Geerts, J., Charles, K. E., Newsham, G. R., \& Marquardt, C. J. G. (2005). Satisfaction with lighting in open-plan offices: COPE field findings. Proceedings of Lux Europa, 10th European Lighting Conference, 414-417. Berlin, Germany. 
Veitch, J.A., Charles, K. E., Newsham, G. R., Marquardt, C. J. G., \& Geerts, J. (2003). Environmental satisfaction in open-plan environments: 5. Workstation and physical condition effects. In IRC-RR-154. https://doi.org/10.4224/20386149

Veitch, J.A., Farley, K. M. J., \& Newsham, G. R. (2002). Environmental Satisfaction in OpenPlan Environments: 1. Scale Validation and Methods. In IRC-IR-844. https://doi.org/10.4224/20386149

Veitch, Jennifer A., Charles, K. E., Farley, K. M. J., \& Newsham, G. R. (2007). A model of satisfaction with open-plan office conditions: COPE field findings. Journal of Environmental Psychology, 27(3), 177-189. https://doi.org/10.1016/j.jenvp.2007.04.002

Vischer, J. (2008). Towards an environmental psychology of workspace: How people are affected by environments for work. Architectural Science Review, 51(2), 97-108. https://doi.org/10.3763/asre.200

Waber, B., Magnolfi, J., \& Lindsay, G. (2014). Workspaces That Move People. Harvard Business Review, 92, 68-77,121.

Wang, Z., Zhao, H., Lin, B., Zhu, Y., Ouyang, Q., \& Yu, J. (2015). Investigation of indoor environment quality of Chinese large-hub airport terminal buildings through longitudinal field measurement and subjective survey. Building and Environment, 94, 593-605. https://doi.org/10.1016/j.buildenv.2015.10.014

World Green Building Council. (2014). Health Wellbeing and Productivity in Offices - The next chapter for green building. Retrieved from https:/www.worldgbc.org/news-media/healthwellbeing-and-productivity-offices-next-chapter-green-building

Xing, Y. F., Xu, Y. H., Shi, M. H., \& Lian, Y. X. (2016). The impact of PM2.5 on the human respiratory system. Journal of Thoracic Disease, 8(1), E69-E74. https://doi.org/10.3978/j.issn.2072-1439.2016.01.19

Yeom, D. J., \& Delogu, F. (2019). Thermal preferences and cognitive performance estimation via user's physiological responses. ARCC Conference Repository, 375-384.

Yildirim, K., Akalin-Baskaya, A., \& Celebi, M. (2007). The effects of window proximity, partition height, and gender on perceptions of open-plan offices. Journal of Environmental Psychology, 27(2), 154-165. https://doi.org/10.1016/j.jenvp.2007.01.004

Zalejska-Jonsson, A., \& Wilhelmsson, M. (2013). Impact of perceived indoor environment quality on overall satisfaction in Swedish dwellings. Building and Environment, 63, 134 144. https://doi.org/10.1016/j.buildenv.2013.02.005 


\section{Glossary}

$\begin{array}{ll}\text { ANN } & \text { Artificial neural networks } \\ \text { ANOVA } & \text { Analysis of variance } \\ \text { ANSI } & \text { American National Standards Institute } \\ \text { ASA } & \text { Acoustical Society of America } \\ \text { ASHRAE } & \text { American Society of Heating, Refrigeration, and Air Conditioning Engineers } \\ \text { BLR } & \text { Binary logistic regression } \\ \text { CBE } & \text { Centre for the Built Environment } \\ \text { CRT } & \text { Cathode-ray tube } \\ \text { IAQ } & \text { Indoor air quality } \\ \text { IEQ } & \text { Indoor environment quality } \\ \text { IESNA } & \text { Illuminating Engineering Society of North America } \\ \text { LEED } & \text { Leadership in Energy and Environmental Design } \\ \text { NICE Cart } & \text { NRC Indoor Climate Evaluator Cart } \\ \text { NRC } & \text { National Research Council Canada } \\ \text { PCA } & \text { Principal component analysis } \\ \text { PM2.5 } & \text { Particulate matter with diameter less than } 2.5 \text { micrometers } \\ \text { PMV } & \text { Predicted Mean Vote } \\ \text { POE } & \text { Post-occupancy evaluation } \\ \text { PPD } & \text { Predicted Percentage of Dissatisfied } \\ \text { SBS } & \text { Sick building syndrome } \\ \text { SEM } & \text { Structural equation modelling }\end{array}$

